\title{
Analyse der prozessabhängigen \\ Kosten der mechanisierten Buchenaufarbeitung
}

\author{
Dissertation \\ zur Erlangung des Doktorgrades (Dr. forest.) \\ der Fakultät für Forstwissenschaften und Waldökologie \\ der Georg-August-Universität Göttingen
}

vorgelegt von

Fabian Böttcher

geboren am 22.04.1992 in Leinefelde

Göttingen, November 2020 
1. Gutachter: Prof. Dr. Bernhard Möhring

2. Gutachter: Prof. Dr. Dirk Jaeger

Tag der mündlichen Prüfung: 10.02.2021 


\section{Danksagung}

Die vorliegende Arbeit entstand im Rahmen des Forschungsprojekts „GerLau (Verwertungsorientierte Untersuchungen an geringwertigen Laubholz-Sortimenten zur Herstellung innovativer Produkte)“, einem vom Bundesministerium für Ernährung und Landwirtschaft (BMEL) geförderten Verbundvorhaben der Georg-August-Universität Göttingen, der Nordwestdeutschen Forstlichen Versuchsanstalt (NW-FVA) und dem Fraunhofer-Institut für Holzforschung - Wilhelm-Klauditz-Institut (WKI). Für die uneingeschränkte Unterstützung bei den Außenaufnahmen möchte ich den Mitarbeitenden des Niedersächsischen Forstamtes Münden sowie des Hessischen Forstamtes Reinhardshagen danken. Besonders hervorzuheben sind hierbei die Maschinenführer, die durch ihre Kooperation hauptsächlich dazu beigetragen haben, dass ich meine Fragestellung in diesem Umfang verfolgen konnte.

Ich möchte an dieser Stelle all denjenigen danken, die in den vergangenen Jahren durch ihre inhaltliche und emotionale Unterstützung zum erfolgreichen Abschluss meines Promotionsvorhabens beigetragen haben:

Allen voran ist hier mein Doktorvater Prof. Dr. Bernhard Möhring zu nennen, der es mir ermöglichte, mich wissenschaftlich weiterzuentwickeln und der die anstoßenden Gedanken für meine Arbeit gab. Vielen Dank für die stets offene Tür und das angenehme Arbeitsklima.

Danken möchte ich in diesem Zuge auch meinem Zweitgutachter Prof. Dr. Dirk Jaeger der durch seine wertvollen Hinweise dazu beigetragen hat die Argumentation meiner Arbeit weiter zu stärken.

Prof. Dr. Carola Paul übernahm die Drittbetreuung meiner Arbeit. Vielen Dank für das stets hilfreiches Feedback und die ansteckende positive Art der Problembewältigung. Ein besonderer Dank gebührt weiterhin Dr. Kai Husmann, der mich auf die verschiedensten Weisen in dieser Arbeit unterstützt hat - als Freund, Bürokollege und Statistikexperte. Fritz Hofheinz und Volker von Groß danke ich für Ihre unermüdliche Hilfe bei den AuBenaufnahmen und die schöne gemeinsame Bürozeit.

Für den täglichen Austausch und die unterhaltsamen Stunden vor und nach Feierabend möchte ich meinen Kollegen und insbesondere Dr. Roman Koster und Johannes Wildberg danken. Die gemeinsame Zeit mit euch war mir eine Freude. 
Danksagung

Der abschließende Dank gebührt meiner Anne: Ohne deine Unterstützung hätte ich diese Arbeit ganz sicher nicht zu Ende gebracht - danke, dass du in allen Lebenslagen an meiner Seite stehst.

Göttingen, im März 2021

Fabian Böttcher 


\section{Zusammenfassung}

Im Fokus dieser Arbeit steht die Analyse der prozessabhängigen Kosten der mechanisierten Buchenaufarbeitung im Rahmen eines forstlichen Produktivitätsmodells. Obwohl zahlreiche Modelle zur Erklärung der mechanisierten Aufarbeitungszeit existieren, beschränken sich diese vorzugsweise auf Nadelholzbestände oder aber gelten nur für schwächere Buchen- bzw. Laubholzbestände. Dieses Vorgehen ist kritisch zu betrachten, da der flächenmäßige Anteil an Buchenbeständen in Deutschland sowie deren durchschnittliche Vorräte und Bestandesalter tendenziell immer weiter ansteigen (BMEL, 2014, S. 16). Weiterhin erlauben die technischen Entwicklungen der letzten Jahre eine zunehmende Harvester-Nutzung auch in stärker dimensionierten Buchenbeständen (Kleinschmit, 2015, S. 43) - es fehlen jedoch Modelle, die den Prozess und speziell die prozessabhängigen Kosten der mechanisierten Aufarbeitung von Buchen über $40 \mathrm{~cm}$ BHD beschreiben. Hinzu kommt, dass über den Einfluss vieler (potentieller) Kostentreiber der mechanisierten Buchenaufarbeitung keine Erkenntnisse bestehen, obwohl bei der Ernte stärkerer Bäume erwartungsgemäß andere Kostentreiber eine relevante Rolle spielen als bei der Ernte schwächerer Bäume.

An dieser Stelle setzt die vorliegende Arbeit an, wobei das Prinzip des Stückvolumengesetzes in die Erstellung eines Produktivitätsmodells integriert wurde. Es konnte gezeigt werden, dass die grundlegenden Erkenntnisse von Hilf (1928) auch für moderne forstliche Prozesse gelten. Die präsentierten Befunde dieser Arbeit lassen sich dabei wie folgt zusammenfassen: Die Produktivität der mechanisierten Buchenaufarbeitung wird vorrangig durch das Baumvolumen beeinflusst. Mit zunehmenden Baumvolumen steigt die Produktivität bis zum Punkt der Leistungsgrenze (40 bis $50 \mathrm{~cm}$ BHD) an und sinkt im Zuge der negativen Grenzproduktivität danach wieder ab. Die Stammqualität und der ausgebildete Kronentyp bilden neben dem Baumvolumen die wichtigsten morphologischen Kostentreiber für die mechanisierte Buchenaufarbeitung. Eine steigende Komplexität der Aushaltung vermindert die Aufarbeitung nur geringfügig, führt aber zu deutlich stärkeren Produktivitätseinbußen für die vor- und nachgelagerten Prozesse. Weiterhin erscheint es dienlich, neben einem Gesamtmodell des Aufarbeitungsprozesses die einzelnen Aktivitäten separat zu analysieren, um auf diese Weise zusätzliche signifikante Kostentreiber zu identifizieren.

Die Ergebnisse zeigen - insbesondere vor dem Hintergrund des zunehmenden Anteils an Buchenbeständen in den deutschen Wäldern - die große Relevanz sowie den Bedarf von 
Zusammenfassung

Forschungsarbeiten zu dieser Thematik. Die Arbeit verdeutlicht, dass es gewinnbringend ist, forstliche Prozesse im Detail zu analysieren, da sich speziell in der Analyse der Aktivitäten die wirkliche Stärke der Kostentreiber identifizieren lässt. Ein besonderes Augenmerk zukünftiger Forschungsarbeiten sollte im Besonderen auf den Einfluss des Harvestersystems, speziell im Kontext auftretender Zusatzaktivitäten, gerichtet werden. Zusammenfassend lässt sich festhalten, dass die Dissertation einen ersten Schritt zur Beantwortung der bestehenden Forschungslücken darstellt und ein Modell schafft, welches sowohl in der Theorie als auch Praxis sinnvoll zur Vorhersage der prozessabhängigen Kosten der mechanisierten Buchenaufbereitung genutzt werden kann. 


\section{Summary}

This dissertation focuses on the analysis of the process-dependent costs of mechanized beech processing within a productivity model. Although numerous models exist that describe the mechanized processing time, these models are commonly limited to coniferous timber or only apply to weaker dimensions of beech or hardwood stands. Since beech stands in Germany tend to increase in terms of area and standing timber (BMEL, 2014, p. 16), more research in this context is needed. Furthermore, the technical developments of the last years allow an increasing utilization of harvesters also in higher dimensioned beech stands (Kleinschmit, 2015, p. 43). However, models describing the process and particularly the process-dependent costs of mechanized processing of beech over $40 \mathrm{~cm}$ $\mathrm{DBH}$ are missing. In addition, research on the influence of many (potential) cost drivers of mechanized beech processing is scare, even though other cost drivers play a role in the harvest of larger trees.

The present work integrates the principles of the piece-volume-law into a productivity model. The analysis shows that the findings regarding the piece-volume-law of Hilf (1928) also apply to modern forest operations. The results can be summarized as follows: The productivity of mechanized beech processing is mainly influenced by the tree volume. With increasing tree volume, the productivity increases up to the point of 40 to 50 $\mathrm{cm} \mathrm{DBH}$ and decreases in terms of shrinking marginal productivity. Besides the tree volume, trunk quality and crown type are the most important morphological cost drivers for mechanized beech processing. An increasing number of assortments reduces the productivity only slightly but leads to significantly stronger productivity losses for subsequent processes. Furthermore, it seems appropriate to analyse the individual activities separately in addition to an overall model of the process in order to identify additional relevant cost drivers.

The results reveal the relevance of this work as well as a need for further research on this topic. It becomes apparent, that it is economically beneficial to analyse forest processes in detail: Especially in the analysis of the individual activities the impact of the cost drivers can be identified. Future research should focus on the influence of the harvester system, especially in the regard to additional activities. In summary, the dissertation can be seen as a first step to fill the existing research gaps and to create a model that allows to identify relevant cost drivers. For both theory and practice it is a useful tool to predict the process-dependent costs of mechanized beech processing. 



\section{Inhaltsverzeichnis}

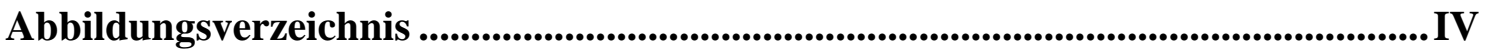

Tabellenverzeichnis .......................................................................................... VIII

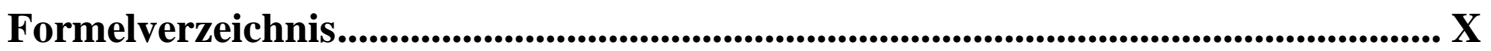

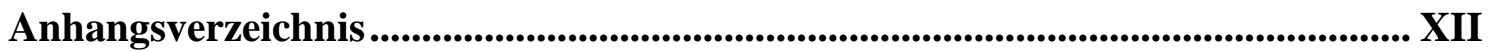

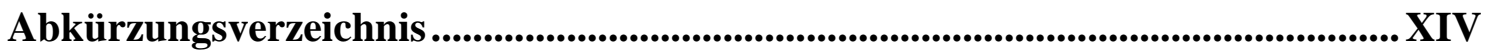

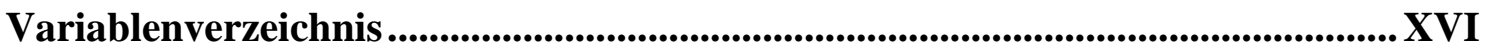

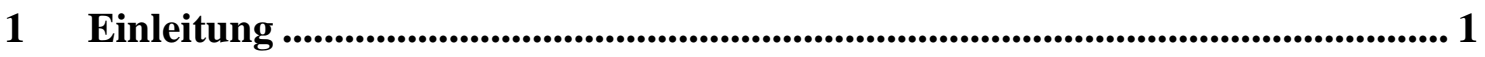

2 Grundlagen der Prozessanalyse ............................................................................................ 6

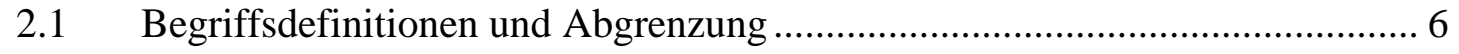

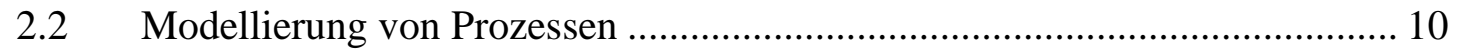

2.3 Die Holzbereitstellungskette als logistisches System................................... 13

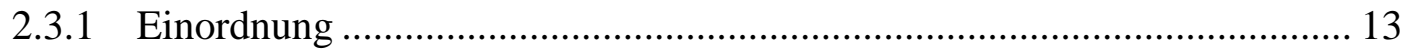

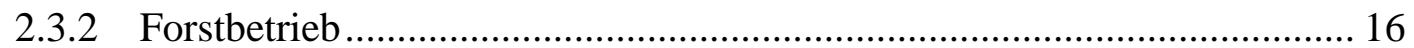

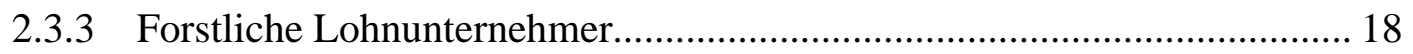

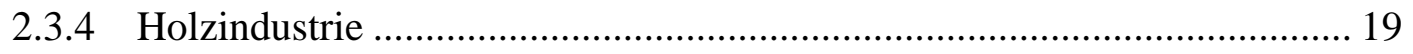

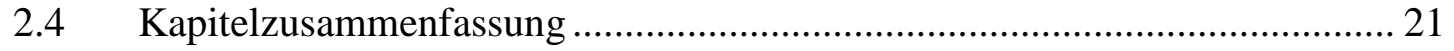

3 Konzepte zur Analyse prozessabhängiger Kosten................................................... 23

3.1 Die Prozesskostenrechnung als Konzept innerhalb des betrieblichen

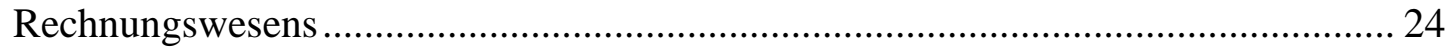

3.2 Prozesskostenrechnung innerhalb der forstlichen Betriebswirtschaftslehre .. 29

3.3 Leistungsuntersuchungen innerhalb der forstlichen Arbeitswissenschaft...... 30

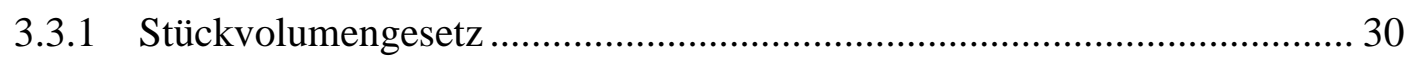

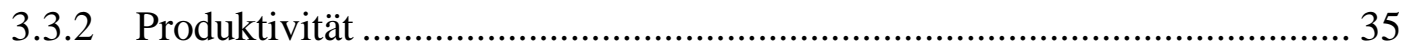

3.3.3 Leistungsbeeinflussende Faktoren ............................................................ 39

3.4 Forstliche Arbeitszeitstudien .................................................................... 41

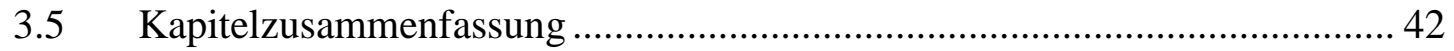

4 Stand, Entwicklung und Herausforderungen der mechanisierten Aufarbeitung von Buchenrohholz ............................................................................................................................ 44 
Inhaltsverzeichnis

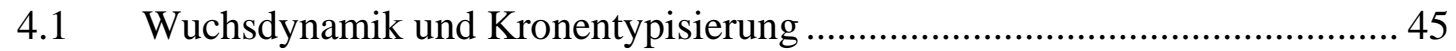

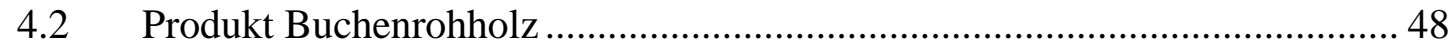

4.3 Arbeitsverfahren der Buchenholzernte …..................................................... 51

4.4 Produktivitätsmodelle der mechanisierten Holzaufarbeitung ........................ 53

4.4.1 Modelle auf der Basis von Bestandesdaten ............................................. 53

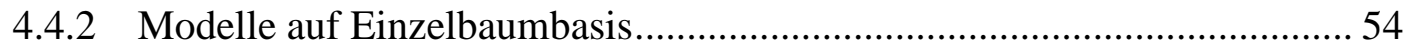

4.4.3 Bedeutung von leistungsbeeinflussenden Faktoren................................. 55

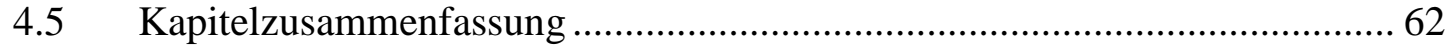

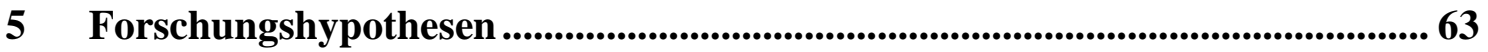

6 Material und Methoden ..................................................................................66

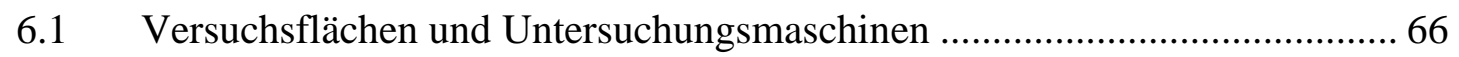

6.2 Aktivitäten der mechanisierten Buchenaufarbeitung ................................... 69

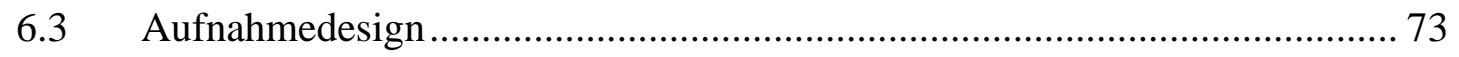

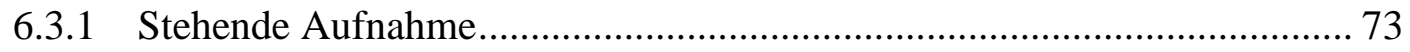

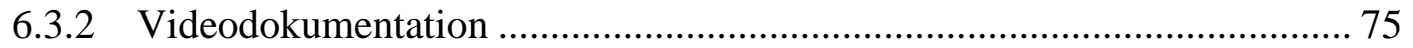

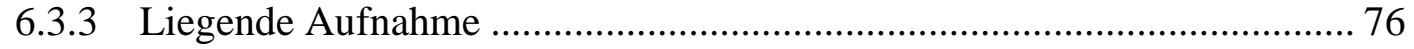

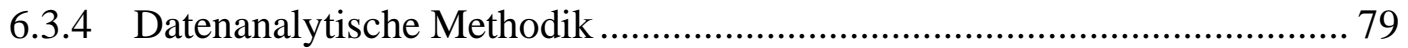

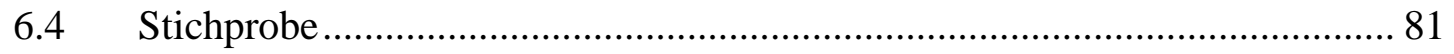

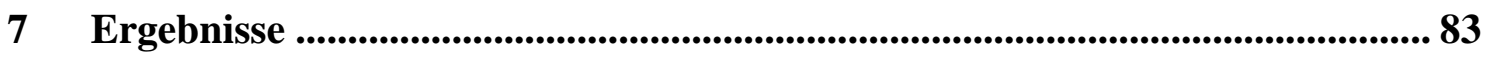

7.1 Modellierung der Aktivitäten ……......................................................... 83

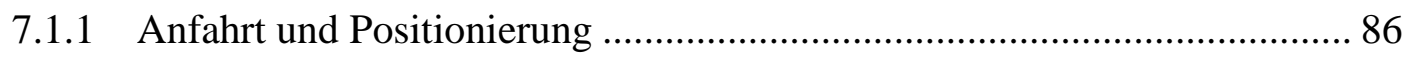

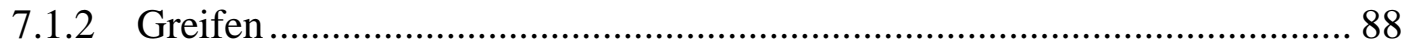

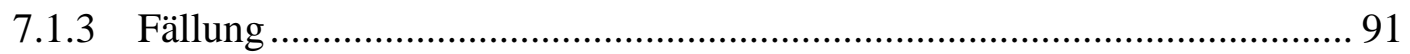

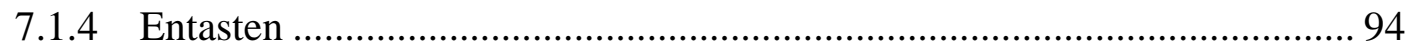

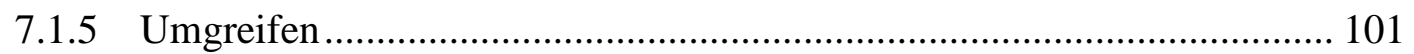

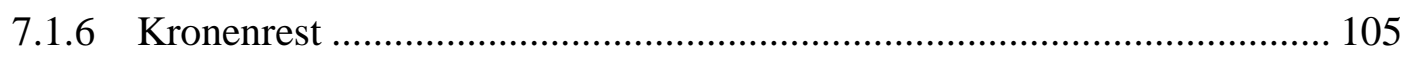

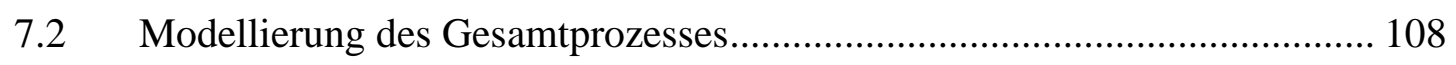

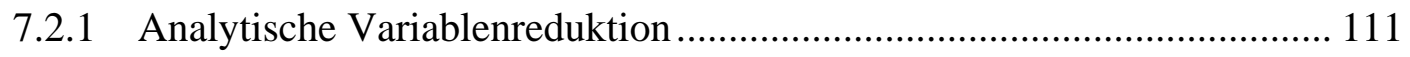

7.2.2 Einfluss der verbleibenden Kostentreiber............................................ 113

7.2.3 Anwendung auf Bestandesebene ...................................................... 117

7.2.4 Einordung in bestehende Produktivitätsmodelle ................................... 121

7.2.5 Summiertes versus direkt geschätztes Produktivitätsmodell................... 125

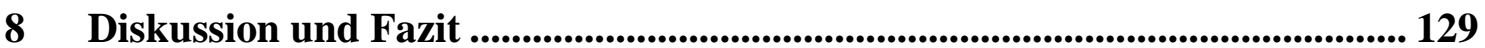




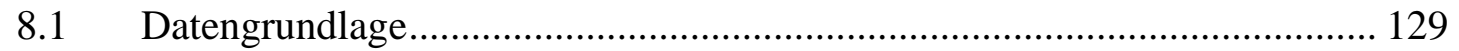

8.1.1 Versuchsflächen und Untersuchungsmaschinen .................................... 129

8.1.2 Stehende und liegende Aufnahme .................................................... 131

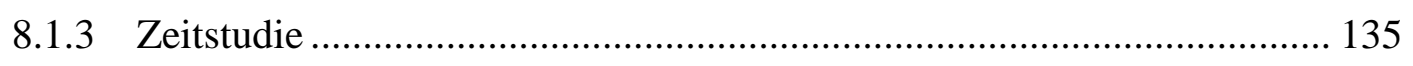

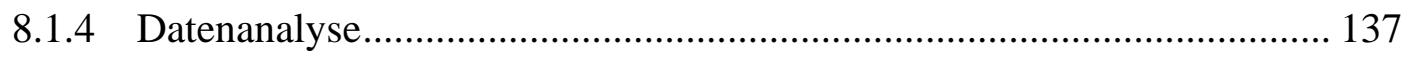

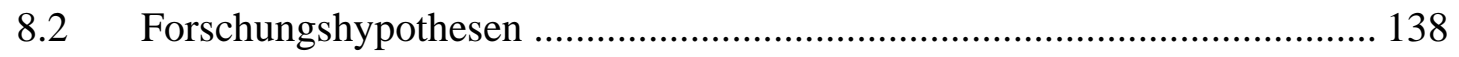

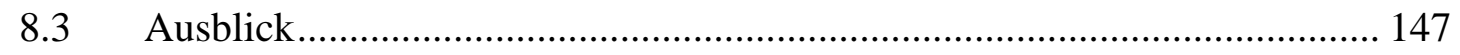

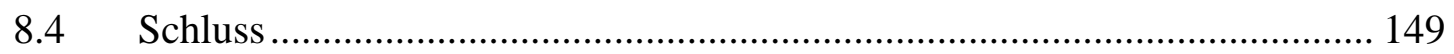

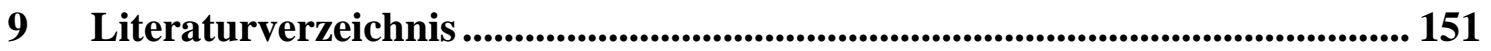

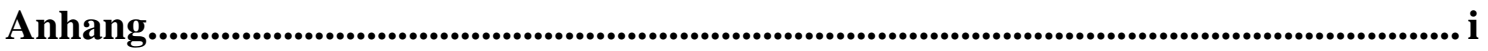

Eigenständigkeitserklärung............................................................................ xiii

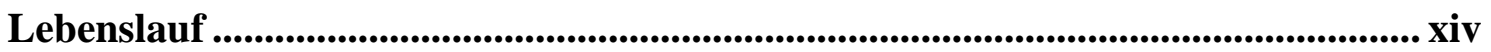




\section{Abbildungsverzeichnis}

Abbildung 1: Thematische Schwerpunkte im Kapitel 2 - Grundlagen der

Prozessanalyse.

Abbildung 2: Übersicht über den Ablauf einer EPK und die notwendigen

Modellierungselemente 12

Abbildung 3: Brüche im Material- und Informationsfluss in der

Holzbereitstellungskette

Abbildung 4: Die Altersklassenverteilung des deutschen Waldes

(getrennt nach Laub- und Nadelbäumen) nach BWI III...................... 17

Abbildung 5: Standorte der Holzindustrie in Deutschland........................................... 20

Abbildung 6: Thematische Schwerpunkte im Kapitel 3 - Konzepte zur Analyse

prozessabhängiger Kosten.

Abbildung 7: Einordnung und Anwendungsbereich der Prozesskostenrechnung innerhalb der Kostenrechnung....

Abbildung 8: Aufarbeitungszeiten je Fm für Fichtenlangholz in Abhängigkeit zum Mittendurchmesser.

Abbildung 9: Bearbeitungszeit je Stück in Abhängigkeit vom Volumen des ausscheidenden Mittelstammes (Stückvolumengesetz).

Abbildung 10: Bearbeitungszeit je Festmeter in Abhängigkeit vom Volumen des ausscheidenden Mittelstammes (Stückvolumengesetz).

Abbildung 11: Produktivität in Abhängigkeit vom Volumen des ausscheidenden Mittelstammes 38

Abbildung 12: Differenzierung der Arbeitszeiten

Abbildung 13: Thematische Schwerpunkte im Kapitel 4 - Stand, Entwicklung und Herausforderungen der mechanisierten Aufarbeitung von

Buchenrohholz.

Abbildung 14: Wipfelschäftiger (links), besenförmiger (mittig) und zwieselförmiger (rechts) Kronentyp bei der Buche

Abbildung 15: Prozessmodell des Einzelbaum- und des Sortimentszyklus für die mechanisierte Buchenaufarbeitung.

Abbildung 16: Beispielaufnahme aus der Harvesterkabine mit der installieren

Videokamera. 
Abbildung 17: Beispielaufnahme eines Raupolters, das bei der mechanisierten Aufarbeitung entsteht.

Abbildung 18: Vergleich des Einzelbaumvolumen über die Funktion nach Bergel (1973) mit der Summe der aufgenommenen Abschnitte (m. R.) im Wald (=Baumvolumen). 78

Abbildung 19: Absoluter und relativer Zeitverbrauch bei der Aufarbeitung der beobachteten Einzelbäume in Abhängigkeit zum Baumvolumen $(0,25$ $\mathrm{m}^{3}$-Stufenbreite) auf Basis der Aktivitäten.

Abbildung 20: Korrelationsmatrix der metrischen Variablen auf Baumebene. 85

Abbildung 21: Abhängigkeit der Anfahrtszeit vom Baumvolumen in den Untersuchungsbeständen. 86

Abbildung 22: Abhängigkeit der Anfahrtszeit von der zurückgelegten Entfernung zu den Entnahmebäumen

Abbildung 23: Oben: Stückzeit für die Aktivität Greifen in Abhängigkeit vom Baumvolumen. Unten: Zeit je $\mathrm{m}^{3}$ für die Aktivität Greifen in Abhängigkeit vom Baumvolumen.

Abbildung 24: Oben: Stückzeit für die Aktivität Fällung in linearer Abhängigkeit vom Baumvolumen. Unten: Zeit je $\mathrm{m}^{3}$ für die Aktivität Fällung in Abhängigkeit vom Baumvolumen. 91

Abbildung 25: Oben: Stückzeit für die Aktivität Fällung in quadratischer Abhängigkeit vom Baumvolumen. Unten: Zeit je $\mathrm{m}^{3}$ für die Aktivität Fällung in Abhängigkeit vom Baumvolumen. 92

Abbildung 26: Stückzeit für die Aktivität Entastung in linearer Abhängigkeit vom Volumen der Abschnitte.

Abbildung 27: Oben: Stückzeit für die Aktivität Entasten in quadratischer Abhängigkeit vom Baumvolumen. Mitte: Zeit je $\mathrm{m}^{3}$ für die Aktivität Entasten in Abhängigkeit vom Baumvolumen. Unten: Produktivität für die Aktivität Entasten in Abhängigkeit vom Baumvolumen. 98

Abbildung 28: Modellierte relative Anteile der Sortenanzahl in Abhängigkeit zum Baumvolumen und der Qualität des Baumes. 100

Abbildung 29: Wahrscheinlichkeit des Umgreifens in Abhängigkeit des Baumvolumens und Kronentyps. 101

Abbildung 30: Boxplot der Umgreifvorgänge pro Baum in Abhängigkeit vom ausgebildeten Kronentyp 102 
Abbildungsverzeichnis

Abbildung 31: Oben: Stückzeit für die Aktivität Umgreifen in linearer Abhängigkeit vom Baumvolumen. Unten: Zeit je $\mathrm{m}^{3}$ für die Aktivität Umgreifen in Abhängigkeit vom Baumvolumen.

Abbildung 32: Modellierte relative Anteile des Kronentyps in Abhängigkeit zum Baumvolumen und der Qualität.

Abbildung 33: Oben: Stückzeit für die Aktivität Kronenrest ablegen in linearer Abhängigkeit vom Baumvolumen. Unten: Zeit je $\mathrm{m}^{3}$ für die Aktivität Kronenrest ablegen in Abhängigkeit vom Baumvolumen. 107

Abbildung 34: Vergleich der beobachteten und der vorhergesagten Werte. Die vorhergesagten Werte wurden durch die Anwendung des vorgestellten Gesamtmodells berechnet (Formel 18).

Abbildung 35: Vergleich der beobachteten und der vorhergesagten Werte. Die vorhergesagten Werte wurden durch die Anwendung des reduzierten Gesamtmodells berechnet (Formel 19).

Abbildung 36: Relative Produktivitätsdifferenzen bei Anwendung des Produktivitätsmodells (Formel 20) in Abhängigkeit zum Baumvolumen und der Baumentfernung

Abbildung 37: Relative Produktivitätsdifferenzen bei Anwendung des

Produktivitätsmodells (Formel 20) in Abhängigkeit der Qualitätsstufe (Oben) mit der Basis: Qualität $1=100 \%$, der Anzahl ausgehaltener Sortimente (Mitte) mit der Basis: Anzahl Sortimente $1=100 \%$ und des Kronentyps (Unten) mit der Basis: Kronentyp „wipfel- schäftig“ = $100 \%$

Abbildung 38: Kosten für die Aufarbeitung (Kosten = 192€/MAS) in Abhängigkeit des Baumvolumens.

Abbildung 39: Vergleich der Produktivitätsmodelle für die mechanisierte Aufarbeitung von Laubholz. 122

Abbildung 40: Vergleich der Produktivitätsmodelle für die mechanisierte Aufarbeitung von Nadelholz mit dem Modell dieser Arbeit (Formel 20).

Abbildung 41: Vergleich der beobachteten und der vorhergesagten Werte. Die vorhergesagten Werte wurden durch die Anwendung des direkt geschätzten Modells berechnet (Anhang 18). 
Abbildungsverzeichnis

Abbildung 42: Vergleich der beobachteten und der vorhergesagten Werte. Die vorhergesagten Werte wurden durch die Anwendung des reduzierten direkt geschätzten Modells berechnet (Formel 24). 


\section{Tabellenverzeichnis}

Tabelle 1: Übersicht über bestehende Produktivitätsmodelle, unterteilt nach der Transformation der unabhängigen Variablen: Baumvolumen.................. 36

Tabelle 2: $\quad$ Übersicht über die leistungsbeeinflussenden Faktoren ............................. 40

Tabelle 3: Übersicht über die Qualitätssortierung von Buchenstammholz ................ 49

Tabelle 4: Beschreibung der aufgenommenen Versuchsflächen.............................. 67

Tabelle 5: Übersicht über die genutzten Harvestersysteme zur Aufarbeitung in den Versuchsflächen.

Tabelle 6: Beschreibung der Aktivitäten und deren definierte Start- und Endzeitpunkte innerhalb des Prozesses der mechanisierten

Buchenaufarbeitung. 71

Tabelle 7: Übersicht über mögliche auftretende zusätzliche Aktivitäten innerhalb der mechanisierten Buchenaufarbeitung. 72

Tabelle 8: $\quad$ Übersicht über die stehend erhobenen Baumvariablen und das jeweilige Aufnahmeverfahren für alle ausgezeichneten Bäume in den Versuchsflächen 74

Tabelle 9: Deskriptive Statistik der erhobenen Bäume. 82

Tabelle 10: Regressionskoeffizient b, Standardfehler SE und Signifikanzwert $\mathrm{p}$ für die lineare Regression des Zeitverbrauchs (Sekunden/Baum) der Aktivität Anfahrt und Positionierung.

Tabelle 11: Regressionskoeffizient b, Standardfehler SE und Signifikanzwert $p$ für die lineare Regression des Zeitverbrauchs (Sekunden/Baum) der Aktivität Greifen 90

Tabelle 12: Regressionskoeffizient b, Standardfehler SE und Signifikanzwert $\mathrm{p}$ für die quadratische Regression des Zeitverbrauchs (Sekunden/Baum) der Aktivität Fällung 93

Tabelle 13: Regressionskoeffizient b, Standardfehler SE und Signifikanzwert $p$ für die lineare Regression des Zeitverbrauchs (Sekunden/Abschnitt) der Aktivität Entasten auf Abschnittsebene. 96

Tabelle 14: Regressionskoeffizient b, Standardfehler SE und Signifikanzwert $p$ für die quadratische Regression des Zeitverbrauchs (Sekunden/Baum) der Aktivität Entasten auf Baumebene 99 
Tabelle 15: Regressionskoeffizient $b$, Standardfehler SE und Signifikanzwert $p$ für die lineare Regression des Zeitverbrauchs (Sekunden/Baum) der Aktivität Umgreifen. 105

Tabelle 16: Regressionskoeffizient b, Standardfehler SE und Signifikanzwert $\mathrm{p}$ für die lineare Regression des Zeitverbrauchs (Sekunden/Baum) der Aktivität Kronenrest ablegen. 108

Tabelle 17: Übersicht über die einbezogenen Variablen innerhalb der Aktivitätsmodelle aus Kapitel 7.1 109

Tabelle 18: Ergebnisse der dritten Bundeswaldinventur für Buchenbestände getrennt nach Altersklasse (rechnerischer Reinbestand). 118

Tabelle 19: Übersicht über den Status der aufgestellten Hypothesen (Kapitel 5)...... 128 


\section{Formelverzeichnis}

Formel 1: Lineares Stückvolumengesetz in Anlehnung an Speidel (1952, S. 37).... 33

Formel 2: Umformung des linearen Stückvolumengesetzes zur Hyperbelform. ....... 34

Formel 3: Produktivität Einzelbaum in Anlehnung an Purfürst (2009, S. 22).......... 36

Formel 4: Produktivität Bestand; $\mathrm{N}_{\mathrm{B}}=$ Anzahl Einzelbäume im Bestand................. 36

Formel 5: Umformung des „,klassischen“ Stückvolumengesetzes im Rahmen einer

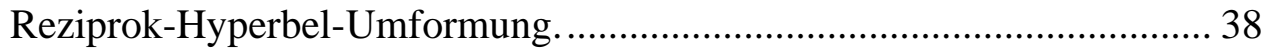

Formel 6: Umformung des quadratischen Stückvolumengesetzes zur Hyperbelform.

Formel 7: Einzelbaum-Volumenfunktion (Vfm) für die Baumart Buche nach Bergel (1973).

Formel 8: $\quad$ Lineare Regressionsfunktion des Zeitverbrauches (Sekunden/Baum) der Aktivität - Anfahrt und Positionierung.

Formel 9: Lineare Regressionsfunktion des Zeitverbrauches (Sekunden/Baum) der Aktivität Greifen

Formel 10: Regressionsfunktion des Zeitverbrauchs (Sekunden/Baum) der Aktivität Fällung

Formel 11: Lineare Funktion zur Erklärung der Entastungszeit auf Abschnittsebene (Sekunden/Abschnitt) 95

Formel 12: Quadratische Funktion zur Erklärung der Entastungszeit auf Baumebene (Sekunden/Baum)

Formel 13: Logistische Regression der Dummy-codierten Variablen Umgreifen..... 102

Formel 14: Geordnete logistische Regression der Anzahl an Umgreifvorgängen je Baum

Formel 15: Lineare Funktion zur Erklärung des Zeitverbrauchs (Sekunden/Baum)

für die Aktivität Umgreifen 105

Formel 16: Lineare Funktion des Zeitverbrauchs (Sekunden/Baum) für das Ablegen des Kronenrestes 108

Formel 17: Funktion des Gesamtprozesses durch die Summierung der Aktivitätsmodelle 108

Formel 18: Kumuliertes Gesamtmodell (Sekunden/Baum) für die mechanisierte Aufarbeitung von Buche. Legende siehe Variablenverzeichnis. 110 
Formel 19: Reduziertes Gesamtmodell (Sekunden/Baum) für die mechanisierte Aufarbeitung von Buche. Legende siehe Variablenverzeichnis.

Formel 20: Produktivitätsmodell der mechanisierten Buchenaufarbeitung 113

Formel 21: Berechnung des Baumvolumens durch die Nutzung des stehenden Einzelbaumvolumens nach Bergel in Vfm (1973).

Formel 22: Geordnete logistische Regression des Kronentyps je Baum 119

Formel 23: Geordnete logistische Regression der Anzahl an ausgehaltenen Sorten je Baum.

Formel 24: Reduziertes direkt geschätztes Gesamtmodell für die mechanisierte Aufarbeitung von Buche auf der Basis des Modells in Anhang 18. Legende siehe Variablenverzeichnis. 


\section{Anhangsverzeichnis}

Anhang 1: Orientierungstabelle für den elektronischen Anhang der

Ausgleichsmodelle.

Anhang 2: Häufigkeitsverteilung der Stichprobe in Abhängigkeit

zum Baumvolumen. ii

Anhang 3: Maximalmodell mit allen theoretischen Variablen für die lineare

Regression des Zeitverbrauchs (Sekunden/Baum) der Aktivität Anfahrt und Positionierung. ii

Anhang 4: Maximalmodell mit allen theoretischen Variablen für die lineare Regression des Zeitverbrauchs (Sekunden/Baum) der Aktivität Greifen ...ii

Anhang 5: Logistische Regression der Eintrittswahrscheinlichkeit für das Auftreten der Zusatzaktivität „Loslassen (L)“ innerhalb der Aktivität Fällung ..........iii

Anhang 6: Maximalmodell mit allen theoretischen Variablen für die quadratische Regression des Zeitverbrauchs (Sekunden/Baum) der Aktivität Fällung .. iv

Anhang 7: Maximalmodell mit allen theoretischen Variablen für die quadratische Regression des Zeitverbrauchs (Sekunden/Baum) der Aktivität Entasten auf Baumebene iv

Anhang 8: Modellierte relative Anteile der Sortenanzahl in Abhängigkeit zum

Baumvolumens und der Qualität in Versuchsfläche 1. $\mathrm{v}$

Anhang 9: Modellierte relative Anteile der Sortenanzahl in Abhängigkeit zum Baumvolumens und der Qualität in Versuchsfläche 3.

Anhang 10: Maximalmodell mit allen theoretischen Variablen für die lineare

Regression des Zeitverbrauchs (Sekunden/Baum)

der Aktivität Umgreifen

Anhang 11: Maximalmodell mit allen theoretischen Variablen für die lineare

Regression des Zeitverbrauchs (Sekunden/Baum)

der Aktivität Kronenrest. vi

Anhang 12: Produktivität der mechanisierten Buchenaufarbeitung in Abhängigkeit vom Baumvolumen vii

Anhang 13: Verteilung der Residuen des kumulierten Gesamtmodells (Formel 18).... vii

Anhang 14: Verteilung der Residuen des reduzierten Gesamtmodells (Formel 19).... viii 
Anhang 15: Absolute Produktivität bei Anwendung des

Produktivitätsmodells (Formel 20) in Abhängigkeit zum Baumvolumen und der Baumentfernung. viii

Anhang 16: Übersicht über die verglichenen Laubholzmodelle zur Überprüfung der Forschungshypothese 5a ix

Anhang 17: Quadratische Regression des BHD (cm) in Abhängigkeit zum Baumvolumen $\left(\mathrm{m}^{3}\right)$ ix

Anhang 18: Übersicht über die verglichenen Nadelholzmodelle zur Überprüfung der Forschungshypothese $5 b$.

Anhang 19: Regressionskoeffizient $b$, Standardfehler SE und Signifikanzwert $\mathrm{p}$ für die quadratische Regression des Zeitverbrauchs (Sekunden/Baum) des analytischen Gesamtmodells für den Prozess der mechanisierten Buchenaufarbeitung xi

Anhang 20: Verteilung der Residuen des direkt geschätzten Gesamtmodells (Anhang 18)

Anhang 21: Verteilung der Residuen des reduzierten direkt geschätzten Modells (Formel 24) xii 


\section{Abkürzungsverzeichnis}

AIC

AZ

$b$

BHD

BL

BPMN

BS

$\mathrm{cm}$

$\mathrm{DBH}$

$D_{g}$

$€$

Efm

Efm m. R.

Efm o. R.

EHT

EPK

EST

$f(\ldots)$

FL

Fm

$\mathrm{H}$

ha

HET

IL

IS

KA

$\mathrm{kNm}$

KT

$\mathrm{kW}$

M
Akaikes Informationskriterium

Allgemeine Zeit (Sachliche und persönliche Verteilzeit)

Regressionskoeffizient

Brusthöhendurchmesser

Energieholz-lang

Business Process Model and Notation

Energieholz-kurz

Zentimeter

Diameter of breast height

Durchmesser des Grundflächenmittelstammes

Euro

Erntefestmeter

Erntefestmeter mit Rinde

Erntefestmeter ohne Rinde

Einheitshauerlohntarif

Ereignisgesteuerte Prozesskette

Erweiterter Sortentarif

Funktion (in Abhängigkeit von)

Stammholzabschnitte

Festmeter ( $\mathrm{m}^{3}$ Holzvolumen)

Höhe

Hektar $\left(10.000 \mathrm{~m}^{2}\right)$

Holzerntetarif

Industrieholz-lang

Industrieholz-kurz

Kronenansatzhöhe

Kilonewtonmeter

Kronentyp

Kilowatt

Arithmetischer Mittelwert 
Abkürzungsverzeichnis

\begin{tabular}{|c|c|}
\hline $\mathrm{m}$ & Meter \\
\hline $\mathrm{m}^{2}$ & Quadratmeter \\
\hline $\mathrm{m}^{3}$ & Kubikmeter \\
\hline MAS & Maschinenarbeitsstunde \\
\hline $\mathrm{MAS}_{0}$ & $\begin{array}{l}\text { Maschinenarbeitsstunde ohne Unterbrechungen } \\
\text { (RAZ) }\end{array}$ \\
\hline MAS $_{15}$ & $\begin{array}{l}\text { Maschinenarbeitsstunde inklusive aller Unterbre- } \\
\text { chungen, die kürzer als } 15 \text { Minuten andauern }\end{array}$ \\
\hline$N$ & Anzahl \\
\hline$N_{B}$ & Anzahl Einzelbäume im Bestand \\
\hline$n s$ & nicht signifikant \\
\hline$O . S D$ & $\begin{array}{l}\text { Arithmetischer Mittelwert zuzüglich der Stan- } \\
\text { dardabweichung }\end{array}$ \\
\hline$p$ & Signifikanzwert \\
\hline PA & Palette \\
\hline$r$ & Korrelationskoeffizient \\
\hline$R^{2}$ & Determinationskoeffizient \\
\hline RAZ & Reine Arbeitszeit \\
\hline$R M S E$ & $\begin{array}{l}\text { Root Mean Square Error (Wurzel der mittleren Feh- } \\
\text { lerquadratsumme) }\end{array}$ \\
\hline RVR & Rahmenvereinbarung für den Rohholzhandel \\
\hline$S$ & Schälholz \\
\hline s $\mid$ Sek & Sekunde \\
\hline$S E$ & Standardfehler \\
\hline $\mathrm{SH}$ & Stammholz \\
\hline ST & Stammholz-lang \\
\hline Stk & Stück \\
\hline $\mathrm{t}$ & Zeit \\
\hline UML & Unified Modeling Language \\
\hline$U . S D$ & $\begin{array}{l}\text { Arithmetischer Mittelwert abzüglich der Stan- } \\
\text { dardabweichung }\end{array}$ \\
\hline$v$ & Stückvolumen (Fm/Stk) \\
\hline Vfm & Vorratsfestmeter \\
\hline
\end{tabular}




\section{Variablenverzeichnis}

\begin{tabular}{|c|c|c|}
\hline Koeffizient & Variable & Beschreibung \\
\hline$\beta_{0}$ & & konstanter y-Achsenabschnitt \\
\hline$\beta_{\text {Entf }}$ & Entf & Entfernung zum Baum [m] \\
\hline$\beta_{V o l}$ & Vol & Baumvolumen $\left[\mathrm{m}^{3}\right]$ \\
\hline$\beta_{\text {Vol1 } 1} ; \beta_{\text {Vol2 }}$ & $\mathrm{Vol} ; \mathrm{Vol}^{2}$ & $\begin{array}{l}\text { Baumvolumen }\left[\mathrm{m}^{3}\right] \text { bei quadratischer } \\
\text { Transformation der Variablen }\end{array}$ \\
\hline$\beta_{\text {Vol.Abs }}$ & $V o l_{A b s}$ & Abschnittsvolumen $\left[\mathrm{m}^{3}\right]$ \\
\hline$\beta_{\text {ZusatzG1 }}$ & ZusatzG $_{1}$ & $\begin{array}{l}\text { Dummy-codierte Variable für auftretende } \\
\text { Zusatzaktivitäten innerhalb der Aktivität } \\
\text { Greifen mit der Ausprägung } 1=\text { Zusatzak- } \\
\text { tivitäten in der Ausführung }\end{array}$ \\
\hline$\beta_{\text {ZusatzF1 }}$ & ZusatzF $_{1}$ & $\begin{array}{l}\text { Dummy-codierte Variable für auftretende } \\
\text { Zusatzaktivitäten innerhalb der Aktivität } \\
\text { Fällung mit der Ausprägung } 1=\text { Zusatzak- } \\
\text { tivitäten in der Ausführung }\end{array}$ \\
\hline$\beta_{\text {ZusatzE0 }}$ & ZusatzE $_{0}$ & $\begin{array}{l}\text { Dummy-codierte Variable für auftretende } \\
\text { Zusatzaktivitäten innerhalb der Aktivität } \\
\text { Entasten mit der Ausprägung } 0=\text { keine Zu- } \\
\text { satzaktivitäten in der Ausführung }\end{array}$ \\
\hline$\beta_{\text {ZusatzK1 }}$ & ZusatzK$_{1}$ & $\begin{array}{l}\text { Dummy-codierte Variable für auftretende } \\
\text { Zusatzaktivitäten innerhalb der Aktivität } \\
\text { Kronenrest mit der Ausprägung } 1=\mathrm{Zu} \text { - } \\
\text { satzaktivitäten in der Ausführung }\end{array}$ \\
\hline$\beta_{S 1}$ & $S 1$ & $\begin{array}{l}\text { Dummy-codierte Variable für das Auftre- } \\
\text { ten der Zusatzaktivität Säuberungsschnitt, } \\
\text { siehe Kapitel } 6.2\end{array}$ \\
\hline$\beta_{Z 1}$ & $Z 1$ & $\begin{array}{l}\text { Dummy-codierte Variable für das Auftre- } \\
\text { ten der Zusatzaktivität Ziehen, siehe Kapi- } \\
\text { tel } 6.2\end{array}$ \\
\hline$\beta_{L 1}$ & $L 1$ & $\begin{array}{l}\text { Dummy-codierte Variable für das Auftre- } \\
\text { ten der Zusatzaktivität Loslassen, siehe } \\
\text { Kapitel } 6.2\end{array}$ \\
\hline$\beta_{A 1}$ & $A 1$ & $\begin{array}{l}\text { Dummy-codierte Variable für das Auftre- } \\
\text { ten der Zusatzaktivität Ast, siehe Kapitel } \\
6.2\end{array}$ \\
\hline$\beta_{M 1}$ & $M 1$ & $\begin{array}{l}\text { Dummy-codierte Variable für das Auftre- } \\
\text { ten der Zusatzaktivität Messen, siehe Ka- } \\
\text { pitel } 6.2\end{array}$ \\
\hline$\beta_{\text {Umgreifvorgänge }}$ & Umgreifvorgänge & $\begin{array}{l}\text { Anzahl der durchgeführten Umgreifvor- } \\
\text { gänge innerhalb der Aufarbeitung }\end{array}$ \\
\hline
\end{tabular}




\begin{tabular}{|c|c|c|}
\hline Koeffizient & Variable & Beschreibung \\
\hline$\beta_{\text {System } 1}$ & System $_{1}$ & $\begin{array}{l}\text { Ausführendes } \text { Harvestersystem mit der } \\
\text { Ausprägung 1, siehe Kapitel } 6.1\end{array}$ \\
\hline$\beta_{\text {System } 2}$ & System $_{2}$ & $\begin{array}{l}\text { Ausführendes } \text { Harvestersystem mit der } \\
\text { Ausprägung 2, siehe Kapitel 6.1 }\end{array}$ \\
\hline$\beta_{\text {System } 3}$ & System $_{3}$ & $\begin{array}{l}\text { Ausführendes Harvestersystem mit der } \\
\text { Ausprägung 3, siehe Kapitel 6.1 }\end{array}$ \\
\hline$\beta_{\text {Gasse }}$ & Gasse & $\begin{array}{l}\text { Entfernung des Baumes zur nächstgelege- } \\
\text { nen Rückegasse [m] }\end{array}$ \\
\hline$\beta_{K T 1}$ & $K T_{1}$ & $\begin{array}{l}\text { Kronentyp mit der Ausprägung } 1=\text { wipfel- } \\
\text { schäftig }\end{array}$ \\
\hline$\beta_{K T 2}$ & $K T_{2}$ & $\begin{array}{l}\text { Kronentyp mit der Ausprägung } 2=\text { zwie- } \\
\text { selförmig }\end{array}$ \\
\hline$\beta_{K T 3}$ & $K T_{3}$ & $\begin{array}{l}\text { Kronentyp mit der Ausprägung } 3=\text { besen- } \\
\text { förmig }\end{array}$ \\
\hline$\beta_{\text {Qualität }}$ & Qualität & $\begin{array}{l}\text { Optische Qualitätseinschätzung des unte- } \\
\text { ren Stammbereiches - Einteilung in eine } \\
\text { von sechs Güteklassen. }\end{array}$ \\
\hline$\beta_{\text {Abschnitte }}$ & Abschnitte & $\begin{array}{l}\text { Anzahl der aufgearbeiteten Abschnitte pro } \\
\text { Baum }\end{array}$ \\
\hline$\beta_{\text {Sorten }}$ & Sorten & $\begin{array}{l}\text { Anzahl der aufgearbeiteten Sortimente pro } \\
\text { Baum }\end{array}$ \\
\hline$\beta_{\text {Herkunft } 1}$ & Herkunft $_{1}$ & $\begin{array}{l}\text { Dummy-codierte Variable für Herkunft } \\
\text { des Abschnittes aus dem Stamm- oder } \\
\text { Kronenbereiches mit der Ausprägung } 1= \\
\text { Stammbereich }\end{array}$ \\
\hline$\beta_{I S}$ & IS & Sortiment Industrieholz kurz \\
\hline$\beta_{P A}$ & $P A$ & Sortiment Palette \\
\hline$\beta_{S}$ & $S$ & Sortiment Schälholz \\
\hline$\beta_{S H}$ & $S H$ & Sortiment Stammholz \\
\hline
\end{tabular}





\section{$1 \quad$ Einleitung}

Der Waldumbau von nadelholzdominierten Wäldern hin zu naturnäheren Laubmischund Laubwäldern spiegelt das Umdenken im waldbaulichen Handeln seit den 1990er Jahren wider und spielt auch in den aktuellen Förderrichtlinien für den Privatwald sowie den Waldbauprogrammen der Länder eine zentrale Rolle (Rumpf \& Petersen, 2008, S. 195). Durch die schweren Stürme der frühen 2000er Jahre wurde diese Strategie relativ schnell flächenwirksam (Fischer et al., 2018, S. 30), sodass die wirtschaftliche Bedeutung von Laubholz stetig wächst und auch in Zukunft ansteigen wird. Alle bedeutenden Aufkommensprognosen deuten darauf hin, dass dieser Trend ungebrochen weitergeht und durch die aktuellen Schadereignisse noch weiter beschleunigt wird (Husmann et al., 2016, S. 899; Schier \& Weimar, 2017, S. 21; Sutmöller et al., 2008, S. 152). Von diesem Umstand profitiert insbesondere die Buche (Rotbuche, Fagus sylvatica L.; Bundesministerium für Ernährung und Landwirtschaft - BMEL, 2014, S. 16): Aufgrund ihrer ökologischen Eigenschaften dominiert sie den Waldumbau und wächst stetig in ihrem Flächenanteil - es erhöht sich jedoch nicht nur der flächenmäßige Anteil an Buchenbeständen, sondern auch die durchschnittlichen Vorräte und die Bestandesalter dieser Baumart steigen kontinuierlich an. So ist der durchschnittliche Buchenbestand in Deutschland mittlerweile im Schnitt 100 Jahre alt (BMEL, 2014, S. 16). All dies wirkt sich auf den Holzmarkt aus (Rosenkranz \& Seintsch, 2017, S. 24) und stellt für die Forstbetriebe eine Herausforderung dar (Spellmann et al., 2007, S. 1249), da diese im Schnitt 80 bis $90 \%$ der Gesamteinnahmen aus der Holzernte erwirtschaften (Warkotsch \& Ziesak, 1998, S. 49). Die zunehmende Bedeutung der Buche verschärft die wirtschaftliche Situation vieler Forstbetriebe, da sich die gesamte technische Produktion von Buchen- im Vergleich zu Nadelholz meist in höheren Kosten (z. B. aufgrund höherer Pflanzkosten, Zaunbau, etc.) bei geringeren Erträgen niederschlägt (Möhring et al., 2008, S. 332). Zudem erfordert die Laubholzernte in der Regel komplexere Verfahren mit längeren Aufarbeitungszeiten und einem größeren Anteil motormanueller Arbeit. Aus diesen Gründen fallen bei Erntemaßnahmen in Laubholzbeständen in der Regel höhere Kosten als in Nadelholzbeständen an und der Personaleinsatz ist intensiver. Ebendies ist problematisch, da die Zahl forstwirtschaftlicher Dienstleistungsunternehmen in Deutschland stetig abnimmt (vgl. Becher, 2015, S. 14; Morat, 2005, S. 75; Wördehoff et al., 2017, S. 17). Darüber hinaus ist die Unfallgefahr bei der motormanuellen Holzernte deutlich 


\section{Einleitung}

höher als bei der mechanisierten Aufarbeitung ${ }^{1}$ (Michels, 2017, S. 211).

All diese Punkte weisen auf einen wachsenden Rationalisierungsbedarf hin, um die Prozesse der Holzbereitstellungskette nutzbringender zu gestalten. Deren effektive Gestaltung sowie die damit verbundene Prüfung der Wirtschaftlichkeit ist Aufgabe der forstlichen Betriebswirtschaftslehre. Wie Speidel (1984, S. 17) formulierte, liegt der „Schwerpunkt betriebswirtschaftlichen Denkens auf den wirtschaftlichen Auswirkungen technischer Maßnahmen und auf der Frage nach den ökonomisch besten Gestaltungsmöglichkeiten der biologischen und technischen Maßnahmen." Eine solche Rationalisierungsmöglichkeit stellt beispielsweise die Entwicklung und Anpassung von kosteneffizienteren Erntemethoden dar. Im Bereich der mechanisierten Aufarbeitung fällt darunter unter anderem der Einsatz von Kranvollerntern, auch Harvester genannt. Diese wurden ursprünglich für die Aufarbeitung schwächer dimensionierter Nadelholzbestände entwickelt und spielen bereits seit längerer Zeit, auch dank technischer Weiterentwicklungen und Innovationen in diesem Bereich, eine zentrale Rolle in der mitteleuropäischen Forstwirtschaft (Purfürst, 2009, S. 1). Harvester werden bei der Buchenholzernte klassischerweise in schwächeren Beständen eingesetzt. Ein Grund hierfür sind die bei älteren $\mathrm{Bu}-$ chen ansetzenden Äste im Kronenbereich, die sich im Laubholz meist nicht durch die Entastungsmesser eines Harvesteraggregats entfernen lassen (u. a. Guglhör, 1994, S. 698; Schorr, 2000, S. 522). In stärkeren Buchenaltholzbeständen haben seilunterstützte Holzernteverfahren und die motormanuelle Holzernte daher immer noch eine große Bedeutung. Die technischen Entwicklungen der letzten Jahre erlauben jedoch eine zunehmende Harvester-Nutzung auch in stärker dimensionierten Buchenbeständen (Kleinschmit, 2015, S. 43), weshalb vermehrt auch dort die Rationalisierungsoption einer mechanisierten Holzaufarbeitung genutzt wird.

Um eine effektive Allokation der knappen Ressource „Arbeitskraft“ zu ermöglichen, werden in der Forstwirtschaft Produktivitätsmodelle genutzt. Diese beschreiben, welche

\footnotetext{
${ }^{1}$ Nach der Definition von Erler \& Dög (2009, S. 17) werden Holzerntesysteme nach ihrem Mechanisierungsgrad in teil-, hoch- und vollmechanisiert unterteilt, wobei der Harvestereinsatz tendenziell den letzten beiden Kategorien zuzuordnen ist. Die Einteilung erfolgt aber immer für das vollständige Holzerntesystem, bestehend aus Holzaufarbeitung und -bringung. Da in dieser Arbeit nur die Buchenaufarbeitung via Harvester im Fokus steht und diesbezüglich keine einheitliche Definition besteht, wird folgend von mechanisierter Aufarbeitung gesprochen, wenn es um den Einsatz von Harvestern geht (vgl. Bacher-Winterhalter, 2003, S. 93).
} 
Leistung für ein bestimmtes Arbeitsverfahren unter festgelegten Rahmenbedingungen $\mathrm{zu}$ erwarten ist. Zusätzlich ermöglichen sie die quantitative Analyse potentieller Kostentreiber, wobei in der arbeitswissenschaftlichen Terminologie der Begriff der leistungsbeeinflussenden Faktoren verwendet wird. Das bekannteste Beispiel für ein solches Modell ist der erweiterte Sortentarif (EST), der im Jahr 1975 als Nachfolger des bisherigen Holzerntetarifs (HET) für die motormanuelle Holzernte entwickelt wurde. Der EST bietet die Möglichkeit, Produktivitätswerte durch prozentuale, kostentreiberabhängige $\mathrm{Zu}$ - und Abschläge zu berechnen. Für die mechanisierte Aufarbeitung von Buche existieren nur wenige Produktivitätsmodelle (u.a. Guglhör, 1994; Pausch, 2002; Schorr, 2000). Die bestehenden Untersuchungen sind zudem veraltet und bilden den aktuellen technischen Stand nicht mehr ab. Im Besonderen existieren keine wissenschaftlichen Aussagen über die mechanisierte Aufarbeitung von Buchen, die einen BHD von über $40 \mathrm{~cm}$ aufweisen. Vor dem Hintergrund immer neuer Harvestersysteme sowie kontinuierlich stärker werdenden durchschnittlichen Erntebäumen ist die Studienlage für Produktionsmodelle der mechanisierten Buchenaltholzaufarbeitung als unzureichend zu bezeichnen. Hinzu kommt, dass über den Einfluss vieler (potentieller) Kostentreiber der mechanisierten Buchenaufarbeitung keine Erkenntnisse existieren, obwohl bei der Ernte stärkerer Bäume erwartungsgemäß andere Kostentreiber eine Rolle spielen.

Dieser Umstand ist kritisch zu betrachten: So ist der Forstbetrieb aufgrund des steigenden Buchenaufkommens und der daraus resultierenden verschärften wirtschaftlichen Bedingungen sowie dem steten Rückgang an forstlichen Arbeitskräften gezwungen, seine Produktivität auf der Fläche zu erhöhen und Prozesse zu rationalisieren. Hierfür sind jedoch zwingend Modelle nötig, die eine forstbetriebliche Entscheidungsfindung unterstützen. Aus diesem Grund besteht das Forschungsinteresse der vorliegenden Arbeit darin, die prozessabhängigen Kosten der mechanisierten Buchenaufarbeitung zu analysieren und mit Methoden der Regressionsanalyse zu quantifizieren. Durch dieses Vorgehen werden die wichtigsten Kostentreiber der mechanisierten Buchenaufarbeitung herausgearbeitet und speziell auch für ein Durchmesserspektrum von 40 bis $65 \mathrm{~cm}$ BHD quantifiziert. Die Arbeit gliedert sich dabei wie folgt:

Die Kapitel zwei bis vier widmen sich der Beschreibung des aktuellen Forschungsstands. Kapitel zwei geht auf die Grundlagen der Prozessanalyse ein. Es wird ein generelles Verständnis über die Grundlagen der Prozessanalyse aus einem Blickwinkel der allgemeinen Organisationslehre sowie im speziellen forstwirtschaftlichen Kontext geschaffen. Dar- 


\section{Einleitung}

über hinaus wird eine Übersicht über die Akteure und Prozesse innerhalb der Buchenholzbereitstellungskette erarbeitet und somit ein Verständnis für die aktuelle und zukünftige Bedeutung der mechanisierten Buchenaufarbeitung geschaffen.

In Kapitel drei werden verschiedene Konzepte zur Analyse prozessabhängiger Kosten mit dem Ziel untersucht, die Kostenanalyse der mechanisierten Buchenaufarbeitung in einen theoretischen Rahmen einzugliedern. Der Blick richtet sich hierbei zunächst auf die Prozesskostenrechnung und deren Umsetzung innerhalb des betrieblichen Rechnungswesens - sowohl im Allgemeinen als auch im speziellen forstlichen Kontext. Im Anschluss erfolgt eine Zusammenfassung der arbeitswissenschaftlichen Methodik der Leistungsuntersuchung. Der Schwerpunkt liegt hierbei insbesondere auf dem Stückvolumengesetz (Hilf, 1928) und dessen Bedeutung hinsichtlich der mechanisierten Aufarbeitung. Den Abschluss des Kapitels bildet eine Übersicht über die Methodik der forstlichen Arbeitszeitstudie.

Kapitel vier enthält eine theoretische Auseinandersetzung mit dem Stand, der technischen Entwicklung und speziell den Herausforderungen der mechanisierten Aufarbeitung in buchendominierten Wäldern. Hierzu werden zuerst die morphologischen Besonderheiten der Buche sowie die daraus resultierenden Eigenschaften des Produktes Rohholz beleuchtet. Nach einem kurzen Exkurs über historische Aufarbeitungsverfahren im Buchenholzeinschlag werden Faktoren aufgezeigt, die den wachsenden Einsatz von Harvestern vorantreiben. Den Abschluss des Kapitels bildet eine Zusammenfassung der existierenden Forschungsarbeiten, die sich mit der Erstellung eines Produktivitätsmodells der mechanisierten Holzaufarbeitung beschäftigt haben. Dabei wird auch die wissenschaftliche Lücke verdeutlicht, die mit der vorliegenden Arbeit geschlossen werden soll.

Basierend auf diesen Überlegungen werden anschließend in Kapitel fünf die zentralen Forschungshypothesen dieser Dissertation hergeleitet, an denen sich die weiteren Analysen orientieren.

Kapitel sechs bildet den Übergang von der Theorie zu den eingesetzten Methoden sowie dem zugrundeliegenden Material. Hierzu werden zunächst die Versuchsflächen und die genutzten Harvestersysteme beschrieben. Darüber hinaus erfolgt eine Darstellung der verschiedenen methodischen Ansätze, welche innerhalb der anschließenden Analyse genutzt werden. Letztlich wird im Zuge des Kapitels auch die verwendete Datengrundlage dieser Arbeit genauer vorgestellt.

Im siebten Kapitel werden die parametrisierten Regressionsmodelle zur Überprüfung der 
Forschungshypothesen vorgestellt. Das erste Unterkapitel zerlegt den Prozess der mechanisierten Buchenaufarbeitung im Sinne der Prozessanalyse in verschiedene Aktivitäten. Diese werden im Rahmen von aktivitätsabhängigen Produktivitätsmodellen einzeln analytisch beschrieben. Mit diesen Modellen sowie einem Gesamt-Produktionsmodell, welches sich aus den Aktivitätsmodellen zusammensetzt, wird die Beantwortung der Hypothesen ermöglicht. Im zweiten Unterkapitel erfolgt die Analyse auf Prozessebene und eine tiefergehende Bearbeitung der Forschungshypothesen. Zusätzlich wird das finale Produktivitätsmodell im Rahmen einer Sensitivitätsanalyse genauer beleuchtet.

In Kapitel acht werden die vorgestellten Ergebnisse in die bestehenden Forschungsarbeiten eingeordnet. Darüber hinaus findet eine Diskussion der genutzten Methoden und des untersuchten Materials statt. Den Abschluss des Kapitels bildet ein Ausblick über den weitergehenden Forschungsbedarf sowie ein Fazit der Arbeit. 


\section{Grundlagen der Prozessanalyse}

Kapitel 2 stellt den theoretischen Einstieg in die Grundlagen der Prozessanalyse im Sinne der allgemeinen Organisationslehre sowie speziell im forstwirtschaftlichen Kontext dar (Abbildung 1). Das erste Unterkapitel widmet sich hierbei den Begrifflichkeiten der Prozessanalyse auf einer allgemeinen Ebene. Im Anschluss daran wird insbesondere die Modellierung von Prozessen beleuchtet und damit der theoretische Rahmen für die lineare Modellierung der Arbeitsprozesse in dieser Arbeit aufgespannt. Das letzte Unterkapitel befasst sich mit dem Prozess der mechanisierten Buchenaufarbeitung im Kontext der vorangegangenen Definitionen. Zusätzlich werden die Akteure und Prozesse innerhalb der Buchenholzbereitstellungskette beleuchtet, um ein Bewusstsein für die aktuelle und zukünftige Bedeutung der mechanisierten Buchenaufarbeitung zu schaffen.

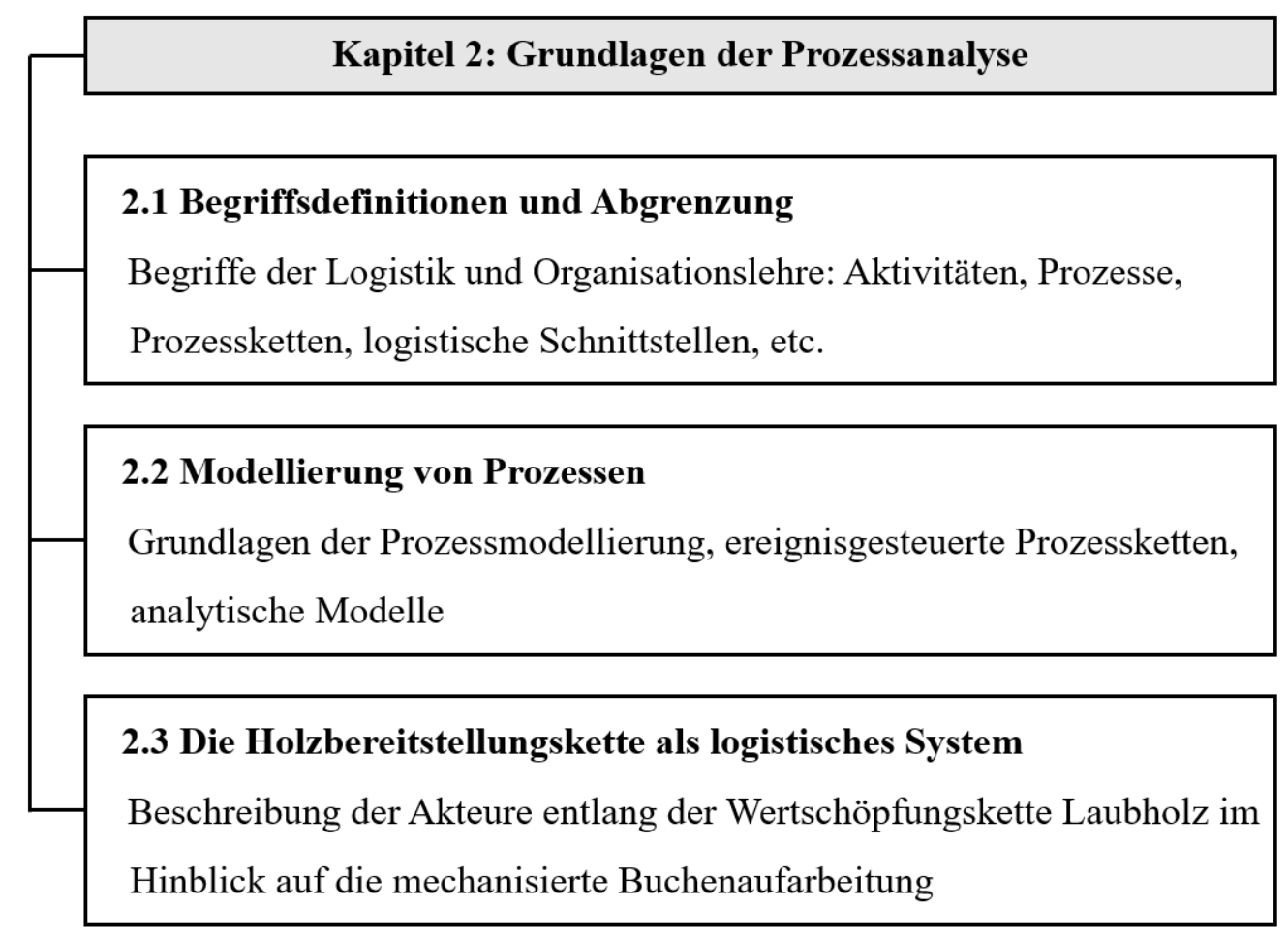

Abbildung 1: Thematische Schwerpunkte im Kapitel 2 - Grundlagen der Prozessanalyse.

\subsection{Begriffsdefinitionen und Abgrenzung}

Der Begriff der Prozessanalyse umfasst die systematische Analyse von Prozessen und die Zerlegung in ihre Einzelteile, mit dem Ziel, Verständnis für den Gesamtprozess zu 
erlangen und Schwachstellen und Verbesserungspotenziale $\mathrm{zu}$ identifizieren (Beach \& Pedersen, 2019, S. 1). Sie stellt den ersten notwendigen Schritt bei der Analyse von Prozesskosten dar. Bevor die Prozessanalyse nachfolgend in der Tiefe behandelt wird, ist es in einem ersten Schritt notwendig, einige Begriffe aus der betriebswirtschaftlichen Organisationslehre ${ }^{2}$ und der Logistik näher zu beleuchten.

Unter einem Prozess versteht man eine zeitlich und räumlich definierte Folge von Aktivitäten $^{3}$, die zueinander in einer Leistungsbeziehung stehen (vom Brocke \& Sonnenberg, 2011, S. 55; Engelmann, 1995, S. 44). Eine Aktivität umfasst einen zielgerichteten Einzelvorgang, wie beispielsweise das Erstellen eines Arbeitsauftrages. Sie bildet die kleinste Einheit der Prozessanalyse und kann als Grundbaustein der Unternehmenstätigkeit betrachtet werden (Baumgarten \& Wiegand, 1996, S. 53). Ein Prozess wiederum meint eine inhaltlich abgeschlossene, zeitliche und sachlogische Abfolge von Aktivitäten, die zur Bearbeitung eines relevanten Objektes notwendig sind (Vossen, 1996, S. 18). Im Controlling und strategischen Management eines Unternehmens werden Prozesse mithilfe von Kennzahlensystemen bewertet, um so beispielsweise die Wettbewerbsfähigkeit einzuschätzen und gegebenenfalls zu erhöhen oder das betriebswirtschaftliche Ergebnis zu verbessern.

Bei einer solchen betriebswirtschaftlichen Prozessart wird häufig auch der Begriff des Geschäftsprozesses verwendet. Dieser wird als eine Folge von Wertschöpfungsaktivitäten mit einem oder mehreren Inputs und einem Kundennutzen-stiftenden Output definiert (Lackes et al., 2018, 2. Absatz). Als zusätzliches Merkmal zeichnet sich ein Geschäftsprozess außerdem durch die Erreichung des Unternehmens- bzw. Organisationszieles aus (Staud, 2013, S. 5). Er kann dabei sowohl mit einem Informationsobjekt als auch mit einem materiellen Objekt verbunden sein (Scholz, 2001, S. 11). Im Falle eines Informationsobjektes beschäftigt sich ein Prozess beispielsweise mit einem Arbeitsauftrag. Dabei bilden alle eingebundenen Aktivitäten, wie das Erstellen oder Übergeben des Arbeitsauftrages, den Prozess. Bei einer Verknüpfung mit einer Ware begleitet der Prozess alle Aktivitäten von Warenerstellung bis Warenausgang (Baumann, 2008, S. 9). Dadurch kann ein Prozess sowohl aus personen-, als auch abteilungs- oder unternehmensübergreifenden Aktivitäten bestehen.

\footnotetext{
${ }^{2}$ Die Begriffe beziehen sich auf das Themenfeld der wirtschaftlichen Leistungserstellung innerhalb der betriebswirtschaftlichen Organisationslehre (Hill et al., 1994, S. 17-18).

${ }^{3}$ Weitere Synonyme: Tätigkeiten, Aufgaben oder Arbeiten (REFA Consulting AG, 2020, 1. Absatz).
} 
Geschäftsprozesse können hinsichtlich ihrer Prozesshierarchie in Kern- und Sekundärprozesse eingeteilt werden. Ein Kernprozess besteht aus Tätigkeiten, die unmittelbar mit dem Erreichen des Unternehmens- bzw. Organisationszieles verbunden sind, beispielsweise bei der Fertigung eines Produktes für einen Kunden. Sekundärprozesse hingegen zeichnen sich durch eine eher unterstützende Funktion aus (z. B. Prozesse innerhalb des Personalmanagements). Dabei kann jeder Prozess als Kunde in Bezug auf seine vorgelagerten Prozesse, wie auch als Lieferant in Bezug auf seine nachgelagerten Prozesse verstanden werden (Baumann, 2008, S. 11).

Eine weitere Zuordnung von Prozessen kann über die Einteilung der gesamten Betriebslogistik in abgetrennte funktionelle Subsysteme erfolgen. Nach Vahrenkamp (2005, S. 10) wird hierbei zwischen den Subsystemen Beschaffungs-, Produktions-, Distributions- und Entsorgungslogistik unterschieden. Die Beschaffungslogistik beschäftigt sich mit dem Material- und Informationsfluss vom Lieferanten in das Unternehmen hinein. Die Produktionslogistik beinhaltet alle Aktivitäten, die sich mit der Vorbereitung, Durchführung und Kontrolle der Produktion einer bestimmten Ware beschäftigen. Die Distributionslogistik beschäftigt sich mit dem Material- und Informationsfluss vom Unternehmen zum Kunden (Vahrenkamp, 2005, S. 85). Dabei entstehen zwangsweise Übergänge zwischen den einzelnen Subsystemen, die als logistische Schnittstellen bezeichnet werden (Feierabend, 1980, S. 59). Diese können sowohl den Materialfluss, also physische Schnittstellen, als auch den Informationsfluss und somit Informationsschnittstellen betreffen. Ein häufiges Ziel der Prozessanalyse ist die Verringerung solcher Schnittstellen zur Vermeidung eines überflüssigen Ressourcenverbrauches.

Ein weiterer wichtiger Punkt der Logistik beschäftigt sich mit der Verknüpfung von Prozessen. Einzelne Prozesse, die in einem logischen Zusammenhang stehen, bilden hierbei entlang eines gleichgerichteten Material- und Informationsflusses Prozessketten. Eine spezifische Form ist die Wertschöpfungskette eines Unternehmens. Sie umfasst sämtliche Fertigungs- und Absatzstufen, von der Rohstoffgewinnung über die Produktion bis hin zum Kundenservice, und schließt die Funktionsbereiche Forschung und Entwicklung, Beschaffung, Fertigung und Absatz mit ein (Kuhn \& Hellingrath, 2002, S. 10). Analysen von Wertschöpfungsketten verfolgen das Ziel, Rationalisierungsmöglichkeiten in allen Bereichen und für alle genutzten Ressourcen aufzudecken (Baumann, 2008, S. 11). Das Augenmerk liegt dabei auf den Aktivitäten und Prozessen, die direkt mit der Kosten- und Wertschöpfungsstruktur in Verbindung stehen. 
In einem direkten Zusammenhang mit der Wertschöpfungskette steht der Begriff des Supply Chain Managements (SCM). Dieser Teilbereich der Logistik wurde erstmals von angloamerikanischen Consulting-Unternehmen in den 1980er Jahren begründet (Houlihan, 1988). Nach Busch und Dangelmaier (2002, S. 4) stellt das SCM ein Netzwerk von verschiedenen Unternehmen dar, welche zusammenarbeiten, um ein bestimmtes Produkt zu einer bestimmten Zeit einem bestimmten Endnutzer zur Verfügung zu stellen. ${ }^{4}$ Ziel einer solchen Betrachtung ist es, die einzelnen Aktivitäten zwar isoliert zu untersuchen, jedoch nicht isoliert zu verbessern, sondern vielmehr Potentiale zur Verbesserung entlang der gesamten logistischen Kette zu finden. SCM beschreibt somit die prozessorientierte Planung und Steuerung der Material- und Informationsflüsse über die gesamte Wertschöpfungskette vom Lieferanten bis zum Kunden. Dabei stehen neben den innerbetrieblichen Prozessen der partizipierenden Unternehmen vor allem die zwischenbetrieblichen Vorgänge im Fokus.

Zuletzt sollen die Begriffe Arbeitsverfahren und Arbeitsaufgabe erläutert werden, die dem Bereich der Arbeitswissenschaft zugeordnet werden können. Eine Arbeitsaufgabe stellt das Ziel einer Handlung dar; unter einem Arbeitsverfahren wiederum versteht man die Art der Bewältigung einer festgelegten Arbeitsaufgabe (REFA - Fachausschuss Forstwirtschaft, 1998, S. 120). Das Arbeitsverfahren beschreibt somit den theoretischen Ablauf eines Prozesses. Im Unterschied dazu besitzt ein Prozess einen konkreten zeitlichen und räumlichen Bezug. Das Arbeitsverfahren kann also als Sollvorgabe für einen Prozess verstanden werden (Erler, 2000, S. 22).

Eine valide Prozessanalyse bedarf ihrer ordnungsgemäßen Durchführung. Die Analyse von Prozessen, speziell die Geschäftsprozessanalyse, dient der Ist-Zustandserfassung und Entwicklung von Sollzuständen eines Prozesses. Hierbei werden qualitative und quantitative Forschungsmethoden eingesetzt. Generell lassen sich zwei unterschiedliche Ansätze ausmachen, die mit der untersuchten Wirkrichtung eines Prozesses zusammenhängen: Im Top-Down-Ansatz werden - von der obersten Unternehmensebene ausgehend verschiedene Prozesse auf Aktivitäten heruntergebrochen. Der Bottom-Up-Ansatz hingegen nimmt erst alle anfallenden Aktivitäten auf und fasst diese erst im Anschluss zu

\footnotetext{
${ }^{4}$ Nach Ellram und Cooper (1990, S. 2) definiert sich SCM als Verknüpfung von wertschöpfenden Prozessen. Fischer (1997, S. 106) hingegen sieht im SCM zu allererst einen Weg der Distribution vom Unternehmen zum Kunden. Harrington (1995, S. 30) beschreibt es letztlich als ein Konstrukt zur Verknüpfung von Material- und Informationsflüssen.
} 
Grundlagen der Prozessanalyse

Prozessen zusammen. Die Wahl des Ansatzes ist besonders hinsichtlich der Analysemethodik ausschlaggebend. Während im Bottom-Up-Ansatz meist Zeitstudien zum Einsatz kommen, werden für eine Prozessanalyse mit Top-Down-Ansatz vorwiegend qualitative Forschungsmethoden wie beispielsweise Experteninterviews genutzt (Krupp, 2006, S. 128).

\subsection{Modellierung von Prozessen}

Zur besseren Verständlichkeit und Abbildung eines Geschäftsprozesses kommen visuelle Modellierungen zum Einsatz. Das Ziel einer Modellierung besteht darin, die Zusammenhänge von Elementen innerhalb eines Systems mit abgesteckten Grenzen zu vereinfachen und systematisch darzustellen (Bruchner, 2004, S. 51). Dieser Systemausschnitt beinhaltet alle relevanten Akteure, Objekte, Prozesse etc. sowie die Beziehung zu anderen Systemen (Imboden \& Koch, 2003, S. 7). Betriebliche Abläufe stellen sich unter genauer Beobachtung jedoch als bemerkenswert komplex und dynamisch heraus (u. a. Möhring, 1994, S. 2; Nagel \& Böttcher, 2019, S. 46). Die Informationen und Zusammenhänge in einem vereinfachten Schema wiederzugeben, ist eine Aufgabe der Modellierung (Baumann, 2008, S. 44). Sie folgt dabei verschiedenen Grundsätzen, auf die nachfolgend in Kürze eingegangen wird (Rosemann et al., 2012, S. 49-50):

Der Grundsatz der Richtigkeit bezieht sich auf die korrekte Darstellung des im Modell abgebildeten Systemausschnittes. Dies betrifft sowohl den Inhalt als auch die Hierarchie der Prozesse. Zusätzlich enthält dieser Grundsatz die richtige Verwendung von Notationsregeln. Der Grundsatz der Relevanz besagt, dass das Modell alle relevanten Sachverhalte darstellt und auf irrelevante Informationen verzichtet (Parsimonie). Ein ordnungsgemäßes Modell sollte außerdem alle Inhalte so darstellen, dass diese vom Betrachter verstanden werden (Grundsatz der Klarheit). Abschließend sollte modellübergreifend eine konforme Anwendung der Modellierungsempfehlungen gewährleistet werden (Grundsatz der Vergleichbarkeit). Gleichbedeutend zu den Grundsätzen werden auch die Prinzipien der Modellierung nach Bernroider und Stix (2004) aufgeführt. Dazu gehören die Prinzipien der Abstraktion, der Partitionierung und der Projektion (vgl. Baumann, 2008, S. 45). 
Diese Grundsätze sind wichtig, da sie unabhängig von der gewählten Modellierungssprache $^{5}$ und dem Einsatzzweck des Modells gelten. Der Zweck eines Modells kann je nach Fragestellung variieren: Zum einen kann die Modellierung einer vollständigen Organisationsdokumentation dienen. Zum anderen können auch Benchmarking oder eine Reorganisation von Prozessen das Ziel einer Prozessmodellierung sein (Rosemann et al., 2012, S. 52-59).

Je nach Einsatzzweck finden verschiedene Modellierungssprachen Verwendung. Grund hierfür ist die Tatsache, dass Prozessmodellierungen in unterschiedlichen Fachgebieten und mit verschieden Einsatzzwecken durchgeführt werden. Die Sprachen unterliegen dabei einer zunehmenden Standardisierung und können als eine Sammlung von Symbolen und Regeln verstanden werden (vom Brocke \& Sonnenberg, 2011, S. 58). ${ }^{6}$ Eine der verbreitetsten Modellierungssprachen ist die Ereignisgesteuerte Prozesskette (kurz: EPK; vgl. Keller et al., 1992, S. 16), die zur grafischen Ablaufmodellierung von Prozessen genutzt wird. Sie wurde im Rahmen eines Forschungsprojektes an der Universität des Saarlandes in Zusammenarbeit mit SAP SE zur semiformalen Beschreibung von Geschäftsprozessen entwickelt (Scheer, 2002, S. 20). Betriebliche Vorgänge können im Rahmen der EPK systematisiert dargestellt werden. Eine EPK verfolgt dabei stets die nähere Beschreibung betrieblicher Prozesse auf Ebene der Tätigkeiten. Das graphische Modell besteht aus verschiedenen Modellierungselementen bzw. Objekttypen (Abbildung 2, Keller et al., 1992, S. 11-13). Den Kern einer EPK bilden dabei Funktionen, die eine fachliche Tätigkeit mit einem klaren Anfang und Ende darstellen. Im Rahmen der Organisationslehre stehen Funktionen synonym für Tätigkeiten (Baumann, 2008, S. 49). Sie enthalten objektspezifische Parameter und Informationen zu Leistung, Kosten und Zeitverbräuchen. Außerdem sind den Funktionen eine (oder mehrere) ausführende Person(en) zugeordnet. Eine EPK beginnt und endet immer mit mindestens einem Ereignis, welches den Zustand der Tätigkeit zu einem bestimmten Zeitpunkt darstellt. Das Ereignis kann dabei Auslöser und/oder Ergebnis einer Funktion sein. Durch Konnektoren werden Funktio-

\footnotetext{
${ }^{5}$ Synonym verwendet Hug (2004, S. 59) auch den Begriff Modelltyp. Dieser wird in anderen Veröffentlichungen aber von der Modellierungssprache abgegrenzt (u. a. Baumann, 2008, S. 45-47; Vötter, 2009, S. 21-25).

${ }^{6}$ Dazu gehören beispielsweise die „Unified Modeling Language (UML)“ oder die „Business Process Model and Notation (BPMN)“.
} 
Grundlagen der Prozessanalyse

nen und Ereignisse miteinander verbunden und in einen zeitlichen und sachlichen $\mathrm{Zu}$ sammenhang gebracht. Dazu gehören neben Verzweigungen oder Zusammenführung auch spezielle Regeln für den logischen Ablauf (Und, Oder, Und/Oder; Keller et al., 1992, S. 14).
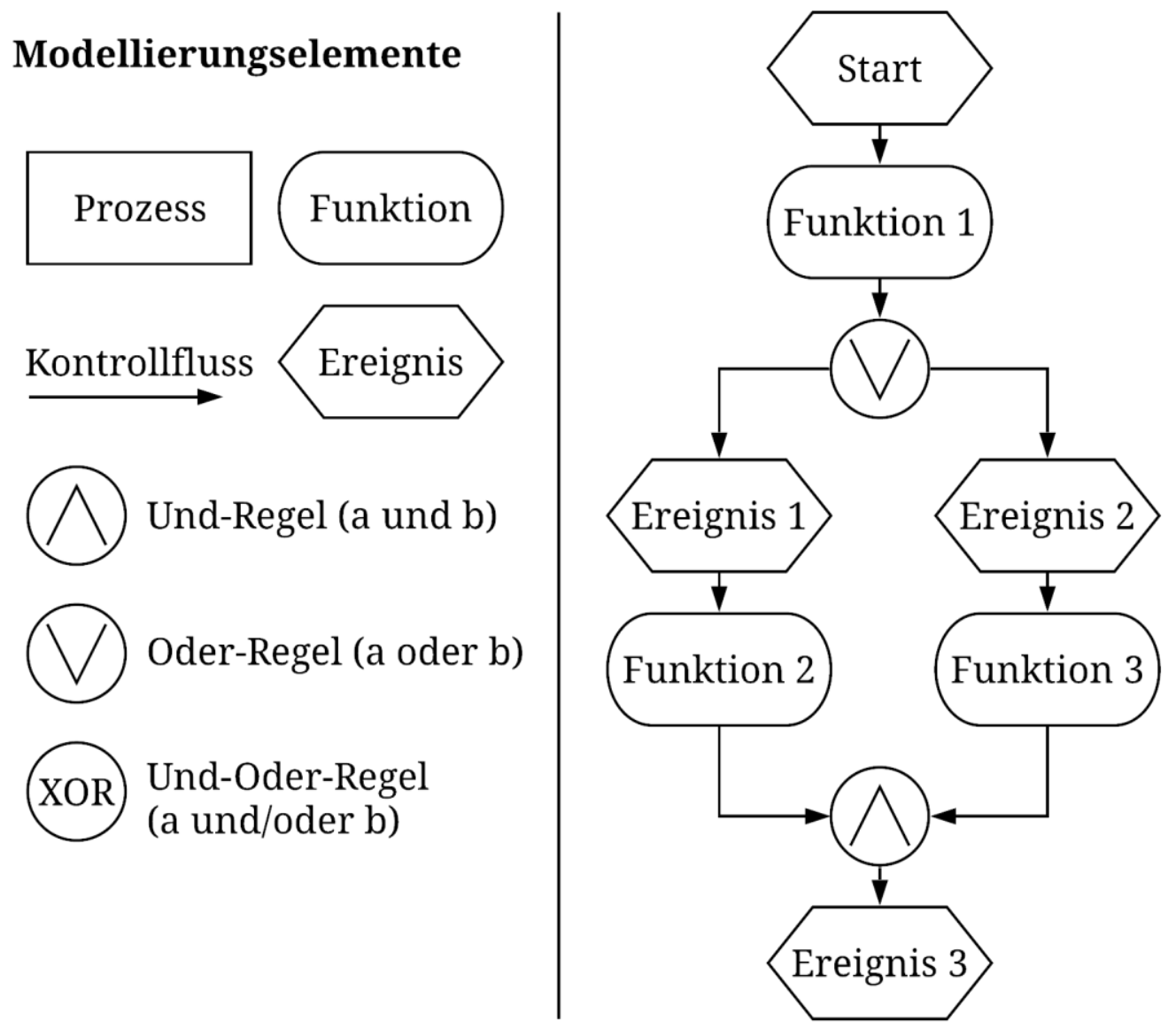

Abbildung 2: Übersicht über den Ablauf einer EPK und die notwendigen Modellierungselemente (eigene Abbildung in Anlehnung an Schwegmann, 1999, S. 76).

Neben der visuellen Modellierung von Prozessen können diese auch analytisch, also über Kennzahlen untersucht werden. Ein solches analytisches Modell besteht nach Bruchner (2004, S. 23) zwingend aus Gleichungen und umfasst mindestens eine abhängige Variable (Ordinate) sowie eine unabhängige bzw. erklärende Variable (Abszisse). Eine be- 
sonders häufig gewählte Form der analytischen Beschreibung einzelner forstlicher Prozesse sind Produktivitätsmodelle ${ }^{7}$. Übertragen auf den Forstbereich beschäftigt sich ein solches Modell zum Beispiel mit der Produktivität eines Harvesters in Abhängigkeit zum BHD der zu entnehmenden Bäume. Ähnliche forstliche Produktivitätsmodelle wurden in der Vergangenheit bereits vielfach untersucht und entwickelt (vgl. Kapitel 3.3). Das bekannteste Beispiel ist der erweiterte Sortentarif (EST), der im Jahr 1975 als Nachfolge zum bisherigen Holzerntetarif $(\mathrm{HET})^{8}$ entwickelt wurde und seitdem zahlreiche Anpassungen durchlaufen hat (Cremer, 2008, S. 14; Dummel et al., 1995, S. 121). Auf der Basis von Zeitstudien wurde ein Modell für die motormanuelle Holzernte entwickelt, welches die Möglichkeit bietet, Produktivitätswerte durch prozentuale $\mathrm{Zu}$ - und Abschläge zu berechnen (Willems, 2015, S. 59). Der Einfluss von weiteren Variablen, wie der Hangneigung oder der Astigkeit der Bäume, wird über die Stärke der $\mathrm{Zu}$ - und Abschläge aufgezeigt. Auch für andere forstwirtschaftliche Prozesse (z. B. mechanisierter Einschlag, Rückung) existieren solche Modelle (im Detail siehe Kapitel 3.3). Produktivitätsmodelle bieten so die Möglichkeit, Prozesse zu zerlegen und die Abhängigkeiten von Variablen zu erklären (Purfürst, 2009, S. 5).

\subsection{Die Holzbereitstellungskette als logistisches System}

\subsubsection{Einordnung}

Zur Analyse der Prozesskosten innerhalb der mechanisierten Aufarbeitung ist es notwendig, die anfallenden Prozesse innerhalb der Holzbereitstellungskette im Sinne der bereits vorgestellten Begrifflichkeiten der Organisationslehre zu zerlegen. Daher beschäftigt sich dieses Unterkapitel mit der Frage, ob sich die Begriffe der allgemeinen Ordnungs- und Logistiklehre auch auf die forstlichen Prozesse innerhalb der Holzbereitstellungskette anwenden lassen. Zusätzlich sollen die Besonderheiten und Probleme einer Prozessanalyse

\footnotetext{
${ }^{7}$ Nach REFA (1978) wird unter Produktivität die Arbeitsproduktivität verstanden, also das Verhältnis von mengenmäßiger Leistung (Output) und dem mengenmäßigen Arbeitseinsatz (Input). Im forstlichen Kontext kann Produktivität als das Verhältnis vom geernteten Holz zum benötigten Zeitverbrauch definiert werden (Purfürst, 2009, S. 22).

${ }^{8}$ Der HET hatte im Jahr 1970 den Einheitshauerlohntarif (EHT) als bisherige Berechnungsgrundlage abgelöst.
} 
Grundlagen der Prozessanalyse

innerhalb forstwirtschaftlicher Fragestellungen beschrieben werden. Alle Beschreibungen sollen der übergeordneten Fragestellung dienen, in welches organisatorische System der Prozess der mechanisierten Buchenaufarbeitung eingebettet ist.

Um die Begrifflichkeiten aus Kapitel 2.1 und ihre Abgrenzung zueinander besser zu verdeutlichen, dient folgendes forstliches Beispiel (in Anlehnung an Engler, 2011, S. 27):

Ein Maschinenführer soll einen Baum mit Hilfe eines Mittelklasse-Harvesters fällen und in festgelegte Sortimente aufarbeiten. Die Arbeitsaufgabe besteht also aus in der Aufarbeitung des Baumes. Der verwendete Arbeitsgegenstand stellt der Baum dar. Das Arbeitsverfahren ist die mechanisierte Holzaufarbeitung mit einem mittelgroßen Harvester (Arbeitsmittel). Der praktische Prozess der Aufarbeitung gliedert sich in verschiedene Aktivitäten, die in einem zeitlichen Ablauf erfolgen: Anfahrt, Greifen, Fällen, Entasten und Aushalten der Sortimente. Neben dem Fällen und Aufarbeiten eines Baumes sind jedoch noch weitere Prozesse wie Bringung, Verkauf und der Transport des Holzes notwendig, um die Wertschöpfungskette der Holzbereitstellung zu beschreiben. Die Holzbereitstellungskette stellt durch ihre Vernetzung von verschiedenen Unternehmen, die sich an ihr beteiligen, auch eine Wertschöpfungskette im Sinne des SCM dar.

Die Holzbereitstellungskette ist demnach ein logistisches System, für das sich die Begriffe und Definition der allgemeinen Logistik- und Organisationslehre anwenden lassen (Vötter, 2009, S. 15). Der nächste Schritt der Prozessanalyse liegt nun in der genauen Abgrenzung der Holzbereitstellungskette zu vor- und nachgelagerten Prozessen und der Einordnung der mechanisierten Aufarbeitung in dieses System.

Basis der Holzbereitstellungskette ist der Forstbetrieb mit seiner biologischen Produktionsfläche. In Anlehnung an Pfohl (2004, S. 179) konnte Baumann (2008, S. 21) zeigen, dass sich die Prozesse innerhalb eines Forstbetriebes in die aus der Organisationslehre bekannten Subsysteme einordnen lassen. Ein wichtiges Merkmal hierbei ist die besondere Form der Produktionslogistik (Pudack, 2006, S. 73). Diese teilt sich in eine biologische und eine technische Produktionsstufe. Die biologische Produktion bildet mit dem Aufbau von Holzvorräten die erste Produktionsstufe; die technische Produktion beschäftigt sich mit der Nutzung dieser Vorräte und wird als zweite Produktionsstufe definiert (Pudack, 2006, S. 73-74). Der Übergang der beiden Produktionsstufen erfolgt durch die Entscheidung, die verfügbaren Holzvorräte zu nutzen. In Anlehnung an bereits bestehende Arbeiten (u. a. Baumann, 2008, S. 22; von Bodelschwingh, 2006, S. 94; Hug, 2004, S. 92; Vöt- 
ter, 2009, S. 40), wird diese Systemgrenze in der vorliegenden Arbeit als Start der Holzbereitstellungskette betrachtet (Abbildung 3).

Zusätzlich zu den Produktionsprozessen des Systems fallen im Forstbetrieb auch Prozesse der Distributions- und der Beschaffungslogistik an ${ }^{9}$, die unmittelbar mit der Holzbereitstellung verknüpft sind (Baumann, 2008, S. 21-22). Diese Prozesse fallen in der Betrachtung dieser Arbeit außerhalb des Systems an und sind folglich nicht versuchsgegenständlich. Neben innerforstbetrieblichen Prozessen, zeichnet sich die Holzbereitstellungkette noch durch betriebsübergreifende Prozessketten aus. Die Holzaufarbeitung (auch: Holzeinschlag), -bringung sowie der Holztransport werden häufig von unabhängigen Unternehmen durchgeführt, die kein Teil des Forstbetriebes sind und somit Schnittstellen zwischen den Subsystemen darstellen. Zusätzlich kommt es hinsichtlich der Holzvermarktung zu Interaktionen zwischen dem Forstbetrieb und der holzbearbeitenden Industrie - diese werden auch als metalogistische Teilsysteme der Holzbereitstellungskette bezeichnet (Baumann, 2008, S. 23). Mit dem Abschluss des Holztransportes und dem rechtlichen Übergang des Holzbesitzes bzw. des Gefahrenüberganges an die holzbearbeitende Industrie endet die Holzbereitstellungskette (Warkotsch \& Ziesak, 1998, S. 49). Im Sinne der Definition aus Kapitel 2.1 weist die Bereitstellung von Holz durch eine Verflechtung von verschiedenen Unternehmen die Eigenschaften einer SCM auf. Die Zusammenarbeit der unterschiedlichen Akteure entlang der Holzbereitstellungskette ist jedoch durch viele logistische Schnittstellen geprägt.

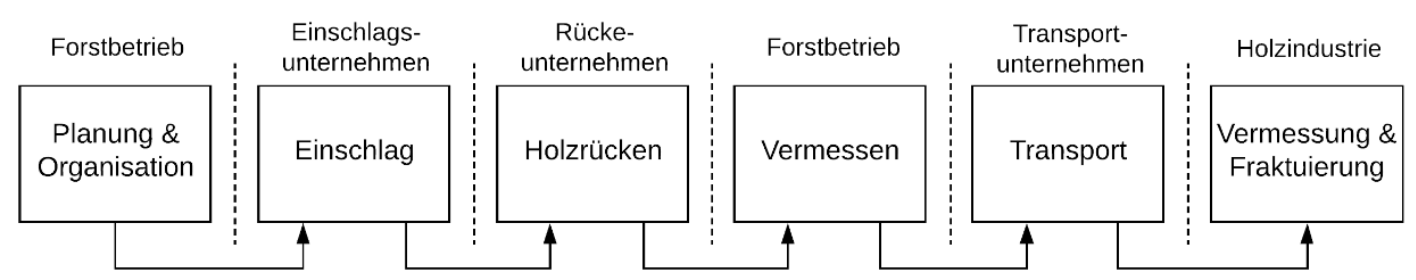

Abbildung 3: Brïche im Material- und Informationsfluss in der Holzbereitstellungskette (eigene Darstellung in Anlehnung an von Bodelschwingh, 2006, S. 94).

\footnotetext{
${ }^{9}$ Distributionslogistik: Kommissionierung des Holzes und Begleitung des Holztransportes. Beschaffungslogistik: Versorgung des Forstbetriebes mit Gütern wie beispielsweise Geräten, Grundstücken oder Maschinen (Baumann, 2008, S. 21-22).
} 
Grundlagen der Prozessanalyse

Um die forstbetriebliche Relevanz dieser Dissertation näher zu beleuchten, werden folgend die relevanten Akteure für die mechanisierte Buchenaufarbeitung sowie deren Schnittstellen zueinander näher beschrieben. Den Abschluss des Kapitels bildet eine Auseinandersetzung mit der Struktur und der Baumartenentwicklung deutscher Forstbetriebe sowie einer Beschreibung der forstlichen Lohnunternehmer und der Holzindustrie in Deutschland.

\subsubsection{Forstbetrieb}

Der Forstbetrieb stellt mit seiner biologischen und technischen Produktion die Basis für die Holzbereitstellungskette dar. In Deutschland finden wir jedoch eine Vielzahl an Waldbesitzformen, die zu differenzierten Forstbetriebsstrukturen führen. Wichtig ist dabei die Trennung von Waldeigentümern, welche die Waldfläche besitzen, und den Forstbetrieben, welche die Waldflächen bewirtschaften. Die Besitzverteilung sowie die räumliche Verteilung des deutschen Waldes allgemein ist in Deutschland sehr heterogen (BMEL, 2016, S. 13). Zum einen ist ein klares Nord-Süd-Gefälle des Waldvorkommens festzustellen. Zum anderen ist die Baumartenzusammensetzung stark von den kleinstandörtlichen Gegebenheiten abhängig. Insgesamt sind rund $32 \%$ der Landesfläche bewaldet. Der Buchenanteil an der gesamten Waldfläche beträgt hierbei rund 15,8 \% mit steigender Tendenz (BMEL, 2016, S. 84-85). ${ }^{10}$ Die Verteilung der Buchenwälder begrenzt sich dabei hauptsächlich auf den mittel- und süddeutschen Raum. Wie Abbildung 4 zeigt, steigt nicht nur der allgemeine Anteil der laubholzdominierten Wälder in Deutschland - sondern werden diese auch immer älter.

Von den 11,4 Mio. ha deutscher Waldfläche befinden sich $48 \%$ in privatem Besitz. Weitere $29 \%$ der Wälder sind Eigentum der Bundesländer, $19 \%$ sind Eigentum von Körperschaften und die restlichen $4 \%$ sind Eigentum des Bundes (BMEL, 2014, S. 9). Der Privatwald ist überwiegend zersplittert und kleinparzelliert. Insgesamt geht man von 1,6 bis 2 Mio. Eigentümern in Deutschland aus (Feil et al., 2018, S. 87; Mrosek et al., 2005a, S. 1113, 2005b, S. 1211; Schraml \& Härdter, 2002, S. 140).

\footnotetext{
${ }^{10}$ Der gesamte Laubholzanteil beträgt 44,5\%. Zwischen der zweiten und dritten Bundeswaldinventur nahm dieser Anteil um 2,8\% zu.
} 
Dem gegenüber stehen die Zahlen der Forstbetriebe, die dieses Eigentum tatsächlich bewirtschaften. Laut Angaben des statistischen Bundesamtes existierten im Jahr 2016 insgesamt 29.408 Forstbetriebe in Deutschland (Statistisches Bundesamt - DESTATIS, 2017, 1. Absatz). ${ }^{11}$ Hinzu kommen Waldeigentümer, die sich in forstlichen Zusammenschlüssen beteiligen. Nach Angaben der Arbeitsgemeinschaft Deutscher Waldbesitzerverbände e.V. (AGDW) sind derzeit fast 400.000 Waldeigentümer in rund 4.500 forstwirtschaftlichen Zusammenschlüssen organisiert (2020, 3. Absatz). Dazu gehören neben Forstgenossenschaften auch Forstbetriebsgemeinschaften.

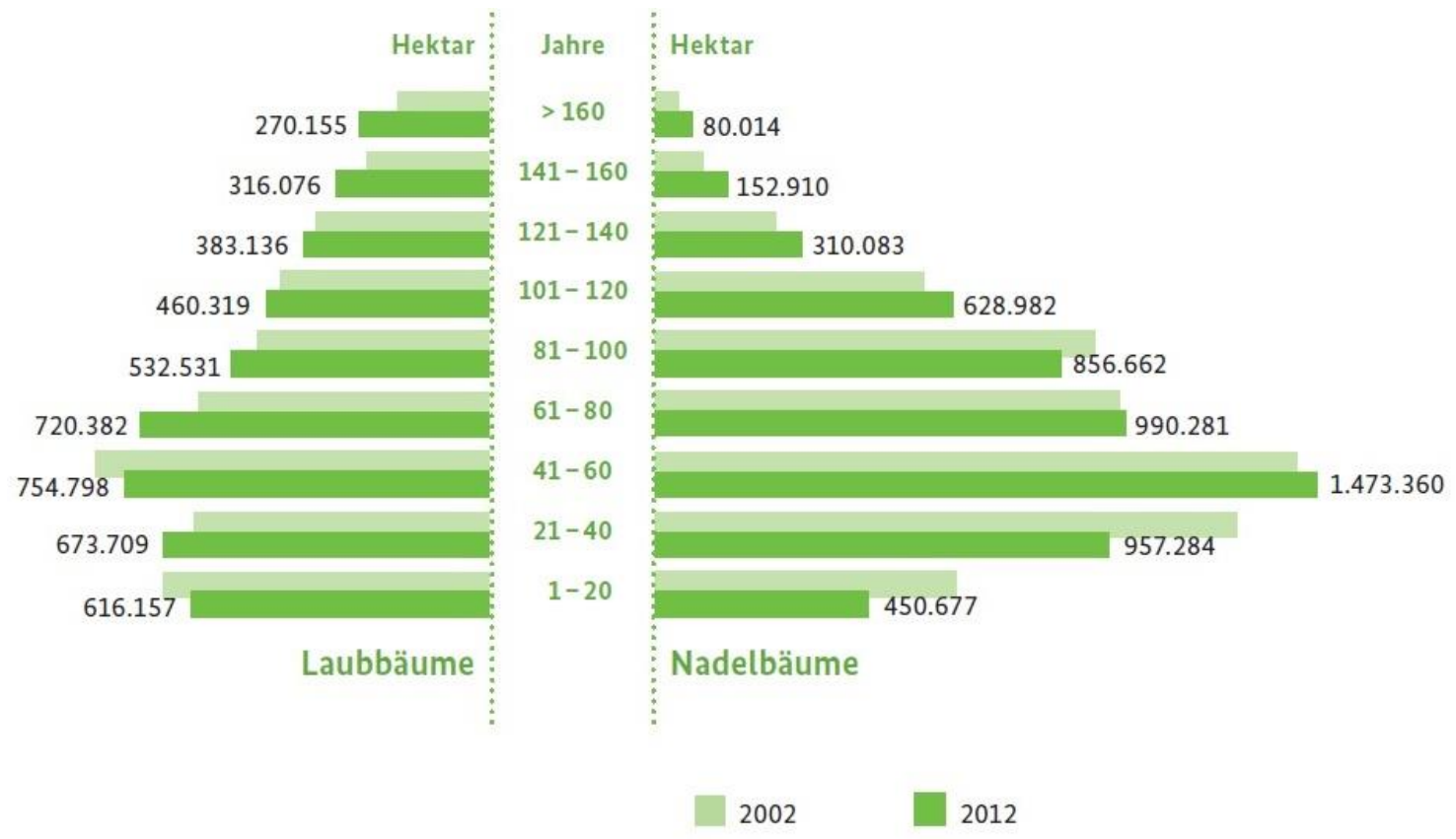

Abbildung 4: Die Altersklassenverteilung des deutschen Waldes (getrennt nach Laubund Nadelbäumen) nach BWI III (Quelle: BMEL, 2016, S. 16).

Im Jahr 2018 betrug der bundesweite Holzeinschlag (gemessen über alle Baumarten) nach Angaben des Bundesamtes für Statistik bei rund 64,6 Millionen Efm o.R. - Buche und sonstiges Laubholz sind darin mit 10,9 Mio. Efm o.R. vertreten (DESTATIS, 2019, 1. Absatz). Nach einer Prognose von Hansen et al. (2008, S. 291) liegt das jährliche Nutzungspotential der Buche bis zum Jahr 2022 bei 14 Mio. bis 20 Mio. Efm o. R. pro Jahr. Zusammenfassend lässt sich somit sagen, die Baumart Buche ist eine Hauptbaumart in

\footnotetext{
${ }^{11}$ Der Hauptanteil der Forstbetriebe bilden Betriebe, die eine relativ kleine Waldfläche bewirtschaften (Waldfläche unter 50 ha ein). Die Anzahl beträgt 19.840 Betriebe.
} 
Deutschland mit jährlich steigendem Flächenanteil. Für den wirtschaftenden Forstbetrieb wird eine Auseinandersetzung mit dieser Baumart daher immer wichtiger.

Für den durchschnittlichen Forstbetrieb stellen die Einnahmen aus der Holzernte 80 bis $90 \%$ der Gesamteinnahmen dar (Warkotsch \& Ziesak, 1998, S. 49). Dieser Aspekt verdeutlicht die großen Potentiale, die sich bei einer näheren Betrachtung der Holzbereitstellungskette ergeben können. Die Identifikation der wichtigsten Kostentreiber innerhalb der Prozesse kann dazu beitragen, die gesamte Holzbereitstellungskette besser zu verstehen und an den relevanten Stellen zu optimieren.

Der Trend geht in vielen Forstbetrieben zunehmend hin zu einer kundenorientierten Holzvermarktung (Baumann, 2008, S. 13). Die Anforderungen der Holzkunden gehen hierbei über die klassischen Warenmerkmale wie Qualität, Abmessungen und Holzpreis hinaus. Stattdessen rücken neue Anforderungen wie die Bereitstellung des Holzes zum gewünschten Lieferzeitpunkt (Just-in-Time-Lieferung) oder die digitale Verarbeitung von Kaufverträgen zunehmend in den Fokus der Kundenorientierung (Böttcher \& Husmann, 2020, S. 9). Durch ein tieferes Verständnis der Holzbereitstellungskette können die notwendigen Maßnahmen für die kundenorientierte Holzaushaltung an den Prozessen vorgenommen werden und deren zusätzlicher Zeit- und Kostenaufwand geschätzt werden.

\subsubsection{Forstliche Lohnunternehmer}

Viele Forstbetriebe wollen bzw. können nicht alle Arbeiten im Wald eigenständig durchführen. Aus diesem Grund sind sie dazu übergegangen, Dienstleistungen auszuschreiben, die von externen Forstunternehmen ausgeführt werden - bestimmte Tätigkeiten werden somit zunehmend aus dem Forstbetrieb ausgelagert. ${ }^{12}$ Die Gründe dafür liegen zum einen in einem geringeren Personalaufwand und zum anderen in der effektiveren Auslastung von Arbeitskapazitäten. Der Schwerpunkt der ausgelagerten Tätigkeiten liegt dabei hauptsächlich im Feld der Holzernte und der Holzrückung bzw. in der technischen Produktionsstufe (Borcherding, 2007, S. 10; Westermayer et al., 2004, S. 1247).

Die deutschen Forstunternehmen zeichnen sich meist durch eine geringe Anzahl von Mitarbeitern aus (eins bis fünf Mitarbeiter bei 90 \% der Betriebe; Westermayer et al., 2004,

\footnotetext{
${ }^{12}$ Synonym dazu wird auch das englische Wort outsourcing verwendet. Dabei werden make-or-buyEntscheidungen zugunsten des buy entschieden und an externe Betriebe vergeben (Oesten \& Roeder, 2012, S. 255).
} 
S. 1248). Laut der 2013 durchgeführten „Clusterstudie der Forst- und Holzwirtschaft der Bundesrepublik Deutschland" existieren in Deutschland 4.073 forstwirtschaftliche Dienstleistungsunternehmen, Tendenz fallend (Becher, 2015, S. 14). So wies eine Untersuchung des Instituts für forstliche Arbeitswissenschaft der Universität Freiburg noch rund 7.300 forstwirtschaftliche Dienstleistungsunternehmen nach (Morat, 2005, S. 75). Neben den klassischen Aufgaben Holzernte und Holzrückung führen die Forstunternehmen auch Tätigkeiten wie Spezialbaumfällung, Entrindung, Hacken oder Zaunbau durch. Eine genaue Erhebung der sich momentan in Deutschland im Einsatz befindenden Kranvollernter existiert hingegen nicht (Purfürst, 2009, S. 36). Zwar führt das Kuratorium für Waldarbeit und Forsttechnik e.V. (KWF) seit 2002 eine jährliche Statistik über die Anzahl der verkauften Neuexemplare durch, über den Gebrauchtmarkt bzw. Privatverkäufe finden sich jedoch keine Zahlen. Studien, die sich mit dem Thema auseinandersetzen, gehen von rund 1.300 bis 1.400 aktuell eingesetzten Harvestern in Deutschland aus (Nick \& Forbrig, 2002, S. 93-99; Nick \& Hofmann, 2005a, S. 97, 2005b, S. 960). Dabei soll die Hälfte aller Maschinen weniger als 5 Jahre alt sein (Baumeister \& von Bodelschwingh, 2004, S. 291-294).

\subsubsection{Holzindustrie}

Eine bedeutende logistische Schnittstelle besteht zwischen dem Forstbetrieb und der Holzindustrie. Sie wird durch die Distribution und Kommissionierung des Holzes gekennzeichnet. Die Holzindustrie teilt sich in zwei Gruppen auf: die holzbearbeitende und holzverarbeitende Industrie. Zur letzten Gruppe gehören die Möbel- und Papierindustrie, welche Produkte für den Endverbraucher herstellen (Bauer, 2006, S. 61). Zur holzbearbeitenden Industrie gehören sowohl die Holzwerkstoffindustrie als auch Säge-, Hobelund Imprägnierwerke - also Unternehmen, die Produkte erzeugen, die zusätzlich verarbeitet werden müssen, bevor sie zum Endverbraucher gelangen (Kroth \& Bartelheimer, 1993, S. 50). Die holzbearbeitende Industrie sowie die Holz- und Zellstofferzeugung sind als erste Absatzstufe des Rundholzes die primären Fahrtziele des Rundholztransportes. Über die genutzten Holzmengen geben Holzrohstoffbilanzen Auskunft. Eine der wichtigsten Datenquellen für den deutschen Raum bildet die Arbeit von Mantau (2018, Abbildung 5). Deutsche Sägewerke schnitten im Jahr 2015 in 2.070 Betriebsstätten rund 36,0 Mio. $\mathrm{m}^{3}$ Stammholz ein (Mantau, 2018, S. 13). Die Struktur der Sägeindustrie ist 
Grundlagen der Prozessanalyse

dabei jedoch stark differenziert. Nur 218 Betriebe sind reine Laubholzsägewerke ${ }^{13}$ mit einem Jahreseinschnitt von 2,3 Mio. Efm o. R. im Jahr 2015. Neben der ausgehaltenen Baumart herrscht eine hohe Differenzierung in der Größe der Sägewerke. Dabei wird jedoch rund dreiviertel der gesamten Einschnittmenge an Stammholz wird in nur 64 Sägewerken verarbeitet ${ }^{14}$ (Döring et al., 2017c, S. 11-14).

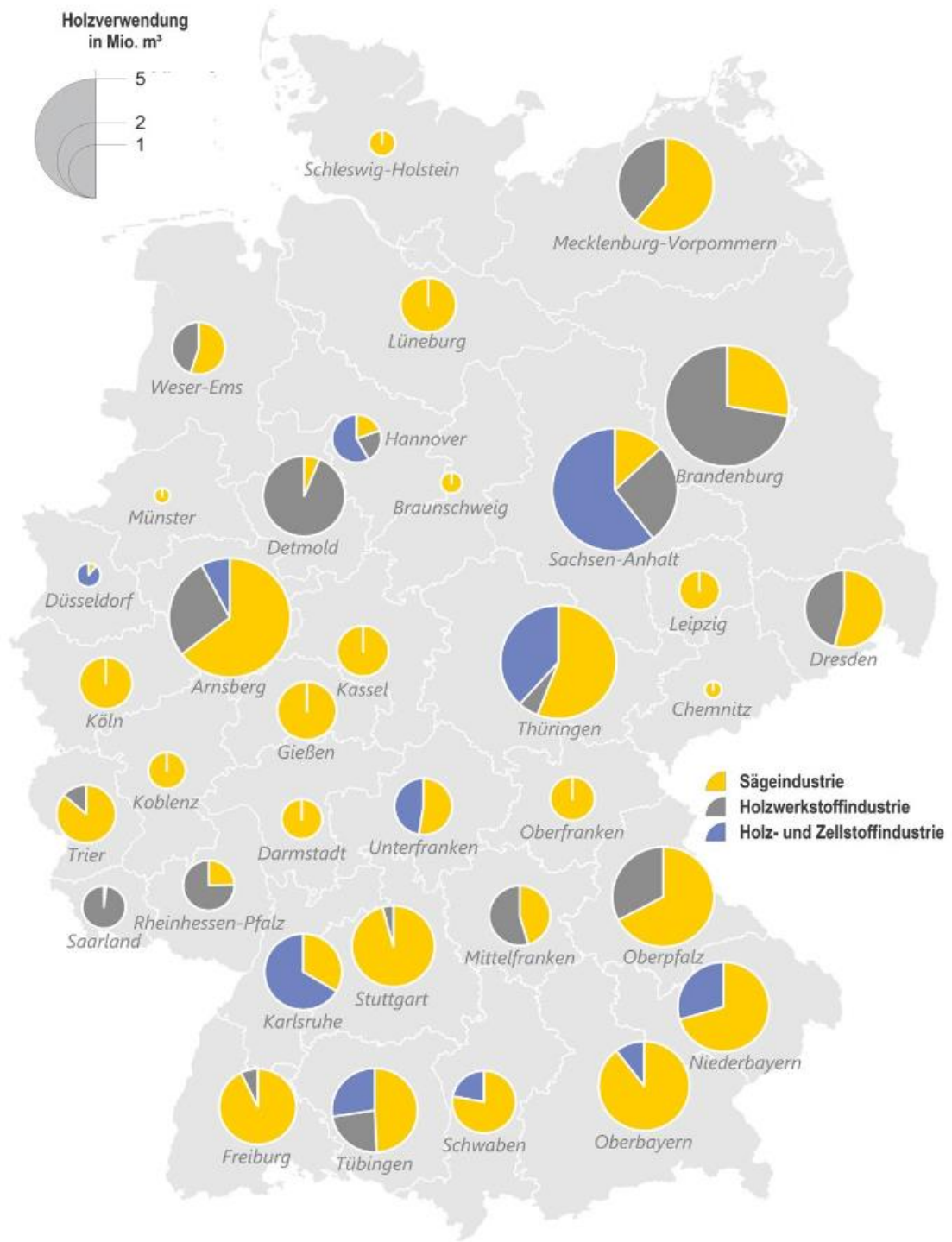

Abbildung 5: Standorte der Holzindustrie in Deutschland (Quelle: Mantau, 2018, S. 14).

\footnotetext{
${ }^{13}$ Zuzüglich 419 Sägebetriebe, die als Mischbetriebe gelten.

${ }^{14}$ Betriebe mit einem Jahreseinschnitt von über $100.000 \mathrm{Fm}$. Von diesen 64 Betrieben schneiden nur zwei Betriebe Laubholz ein (Döring et al., 2017c, S. 11).
} 
Die Holzwerkstoffindustrie besteht zum Großteil aus Standorten, die industriell gefertigte Plattenprodukte herstellen. Im Jahr 2015 wurden in 32 deutschen Betriebsstätten Holzwerkstoffplatten produziert (bei einer Holzverwendung von 15,8 Mio. $\mathrm{m}^{3}$ Rundholz). Der Anteil von Laubholz beträgt dabei rund 2 Mio. $\mathrm{m}^{3}$. Hinzu kommen Anteile von Sägenebenprodukten ${ }^{15}$ oder Altholz, die aber nicht nach Baumartenanteilen aufgeschlüsselt werden können (Döring et al., 2017b, S. 20).

Der letzte Teil der ersten Absatzstufe des Rundholzes besteht aus der Holz- und Zellstoffindustrie. Während bei der Produktion von chemischem Zellstoff das Rohholz in Faser und Lignin aufgetrennt wird, werden diese Bestandteile beim Holzstoff mechanisch aufgetrennt. Dieser Industriezweig benötigte im Jahr 2015 rund 10,0 Mio. $\mathrm{m}^{3}$ Rohholz, mit einem Anteil von 8,3\% Laubholz (Döring et al., 2017a, S. 14).

Es ist erkennbar, dass sich die Holzindustrie immer stärker professionalisiert - speziell in der holzbearbeitenden Industrie wird die Anzahl der Betriebe geringer und der Großteil des Jahreseinschnittes wird durch wenige Unternehmen realisiert. Mit steigender Professionalisierung steigt aber auch der Anspruch an die anderen Akteure der Holzbreitstellungskette. Der Einsatz von modernen Holzerntesystemen kann dazu einen Beitrag leisten.

\subsection{Kapitelzusammenfassung}

Dieses Kapitel widmete sich den zentralen Begriffen der Logistiklehre und der Prozessanalyse. Es wird deutlich, dass der Fokus der Analyse einen Einfluss auf die Beschreibung der Prozessketten nimmt. Um diese zu visualisieren und analytisch beschreiben zu können, ist ihre Modellierung notwendig. Eine spezielle Form der Modellierung stellt das Produktivitätsmodell dar. Es erlaubt die mathematische Zerlegung eines Prozesses und die Quantifizierung von Abhängigkeiten durch erklärende Variablen. Produktivitätsmodelle bilden somit die Brücke zur wirtschaftswissenschaftlichen Thematik der Prozesskostenrechnung.

Den Abschluss des Kapitels bildete die Darstellung der Holzbereitstellungskette als lo-

\footnotetext{
${ }^{15}$ Sägenebenprodukte nehmen mit 39,3 \% den größten Anteil am Holzbedarf für Holzwerkstoffe ein (Döring et al., 2017b, S. 19).
} 
Grundlagen der Prozessanalyse

gistisches System. Dabei konnte gezeigt werden, dass sich die Definitionen der Organisationslehre im forstlichen Kontext wiederfinden und der Prozess der (mechanisierten) Holzaufarbeitung in ein Netzwerk aus Unternehmen eingebettet ist, die im Rahmen der Holzbereitstellungkette kooperieren. Zusätzlich wurde aufgezeigt, dass der Buchenanteil in deutschen Forstbetrieben immer weiter ansteigt. Dabei ergibt sich ein antagonistisches Muster: Während zunehmend mehr forstliche Aufgaben und speziell die Durchführung der Holzernte an Dienstleister ausgelagert werden, nimmt gleichzeitig die Anzahl solcher Unternehmen tendenziell ab. Sie sind daher gefordert, ihre Produktivität auf der Fläche zu erhöhen. Möglichkeiten hierzu bieten der Einsatz und die Weiterentwicklung neuer Maschinensysteme und ein effizienterer Einsatz der begrenzten Ressource Arbeitskraft. Die Grundlage für eine Einschätzung der Effizienz eines solchen Systems bietet die Auseinandersetzung mit den prozessabhängigen Kosten. Aus diesem Grund wendet sich das nachfolgende Kapitel diesem Thema im Detail zu. 


\section{Konzepte zur Analyse prozessabhängiger Kosten}

Kapitel 3 gibt einen theoretischen Überblick über verschiedene Konzepte zur Analyse prozessabhängiger Kosten (Abbildung 6). Das Vorgehen folgt dabei der Frage, in welchen theoretischen Rahmen sich die Kostenanalyse der mechanisierten Buchenaufarbeitung eingliedern lässt. Das erste Unterkapitel beleuchtet hierbei die Prozesskostenrechnung und deren Umsetzung innerhalb des betrieblichen Rechnungswesens aus einer eher allgemeinen Perspektive heraus. Im Anschluss daran erfolgt die Prüfung des bestehenden Forschungsstandes zur Anwendung der Prozesskostenrechnung innerhalb der deutschen Forstwirtschaft.

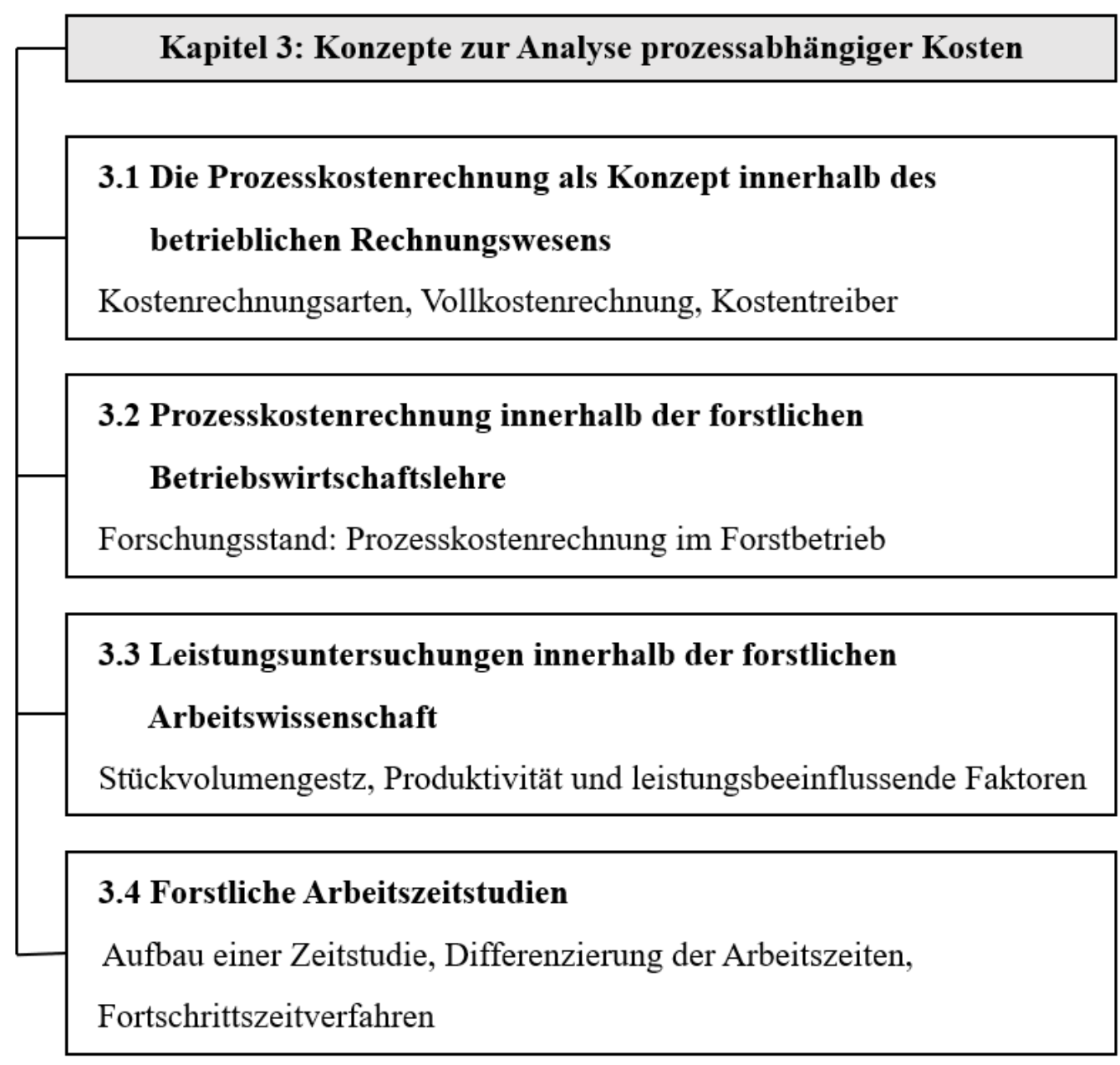

Abbildung 6: Thematische Schwerpunkte im Kapitel 3 - Konzepte zur Analyse prozessabhängiger Kosten.

Das dritte Unterkapitel richtet den Blick auf arbeitswissenschaftliche Perspektive und rückt die Leistungsuntersuchung innerhalb der forstlichen Arbeitswissenschaft in den 
Mittelpunkt. Der Fokus liegt im Besonderen auf dem Stückvolumengesetz und dessen Bedeutung hinsichtlich eines modernen forstlichen Prozesses wie der mechanisierten Aufarbeitung. Den Abschluss des Kapitels bildet eine Übersicht über die Methodik der forstlichen Arbeitszeitstudie, welche die Grundlage für die Erstellung eines Produktivitätsmodells darstellt.

\subsection{Die Prozesskostenrechnung als Konzept innerhalb des betriebli- chen Rechnungswesens}

Das Denken in Prozessen findet neben dem bereits vorgestellten Bereich der Organisationslehre auch Anwendung im Gebiet der Wirtschaftswissenschaften. Die Prozesskostenrechnung bezeichnet dabei ein spezifisches Modellierungsverfahren der allgemeinen Kostenrechnung und gehört damit zum wirtschaftswissenschaftlichen Bereich der Kosten- und Leistungsrechnung bzw. dem internen Rechnungswesen eines Betriebes (Weber, 2018, 3. Absatz). Sie ermöglicht eine beanspruchungsgerechte Verteilung der Gemeinkosten und bildet auch Kosten von indirekten Leistungsbereichen, wie beispielsweise dem Warentransport, ab. Dieser Bereich dient in erster Linie der Informationsbereitstellung, um eine operative Planung im Betrieb und die Kontrolle von Kosten und Erlösen zu ermöglichen (Coenenberg, 1997, S. 220).

Bei der Prozesskostenrechnung handelt es sich um ein Modellierungsverfahren der Vollkostenrechnung, bei der alle effektiven oder planmäßig entstandenen Kosten eines Kostenträgers - also der hergestellten Produkte und Dienstleistungen eines Unternehmens innerhalb einer festgelegten Periode (Jossé, 2012, S. 95) - ermittelt werden sollen. ${ }^{16}$ Bei der Vollkostrechnung erfolgt somit sowohl die Verrechnung der fixen als auch der variablen Kostenbestandteile (Abbildung 7). Als Fixkosten werden Kosten bezeichnet, die unabhängig von der Produktionsmenge in konstanter Höhe anfallen (Coenenberg, 1997, S. 52). Dahingegen zeichnen sich variable Kosten durch die Abhängigkeit von der Produktionsmenge aus und verändern sich proportional zu dieser. Während die Einzelkosten

\footnotetext{
${ }^{16}$ Den Gegensatz dazu bildet die Teilkostenrechnung, mittels derer nur die variablen Kosten untersucht werden und in der die Verrechnung von Fixkosten vermieden wird. Ein Beispiel hierfür ist die Proportionalkostenrechnung (direct costing), welche auch als einstufige Deckungsbeitragsrechnung bekannt ist (Jossé, 2012, S. 131).
} 
direkt auf die Kostenträger verrechnet werden können, müssen die Kosten des indirekten Leistungsbereichs näher analysiert werden, um eine Verrechnung zu ermöglichen.

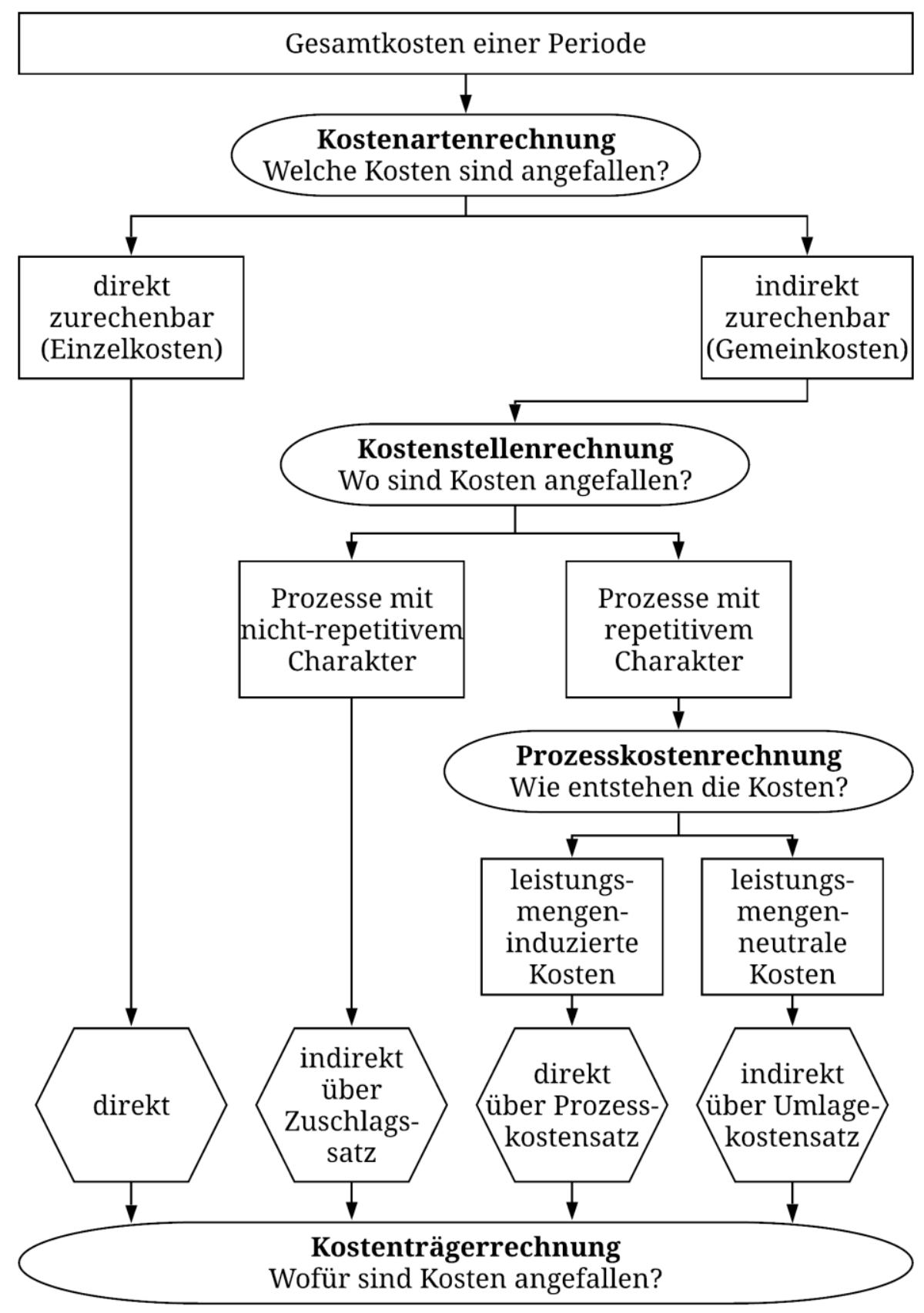

Abbildung 7: Einordnung und Anwendungsbereich der Prozesskostenrechnung innerhalb der Kostenrechnung (eigene Darstellung in Anlehnung an Zimmermann et al., 2003, S. 237).

Im Rahmen der Kostenstellenrechnung werden die Gemeinkosten, getrennt nach ihrer Entstehung (Verursachungsprinzip), in zwei Teilbereiche untergliedert: Prozesse mit einem repetitiven und nicht-repetitiven Charakter. Die Prozesse, die sich nicht wiederholen, 
werden über traditionelle Zuschlagsätze auf die Kostenträger aufgeschlagen. Prozesse mit einem sich wiederholenden Charakter können hingegen durch die Prozesskostenrechnung tiefergehend quantifiziert werden. Die darin zusammengefassten Aktivitäten werden nun auf verursachende Faktoren bzw. Kostentreiber untersucht. Tätigkeiten, die sich auf einen solchen Kostentreiber zurückführen lassen, werden als leistungsmengeninduziert (lmi) definiert und können nun direkt über einen Prozesskostensatz verrechnet werden. Alle anderen Tätigkeiten sind leistungsmengenneutral ( $(\mathrm{mn})$ und werden über einen Umschlagskostensatz auf die Kostenträger aufgeschlagen (Horváth, 2011, S. 489).

Die deutsche Entwicklung der Prozesskostenrechnung geht auf Arbeiten des Elektrokonzerns Siemens (ab ca. 1975) und des Maschinenbauers Schlafhorst (ab ca. 1980) zurück (Schmidt, 2008, S. 239). Eine systematische Aufarbeitung dieser Theorie wurde erstmals von Horváth und Mayer (1989) durchgeführt. Eine ähnliche Entwicklung fand gleichzeitig im englischsprachigen Raum statt, als sich Mitte der 1980er Jahre eine aktivitätsorientierte Form der Kostenrechnung entwickelte, die als Acitivity-based Costing oder auch Cost Driver Accounting beschrieben wird (Horváth, 2011, S. 487; Schmidt, 2008, S. 240; Turney, 1992, S. 20).

Die Gründe für die Notwendigkeit einer Prozesskostenrechnung teilt Coenenberg (1997, S. 220-225) in drei große Bereiche: Erstens liegt der Bedarf an einer prozessorientierten Kostenrechnung in der Veränderung der Wertschöpfungsstruktur begründet. Dazu gehören voranschreitende Computertechnologien und die Einführung von computerintegrierten Produktionssystemen (CIM-Systeme). Während früher eher großvolumige Aufträge von standardisierten Produkten hergestellt wurden (economics of scale), kann nun eine Vielzahl an Produkten in computergestützten Produktionsanlagen wirtschaftlich hergestellt werden (economics of scope). Diese Variantenvielfalt führte zu größeren Anforderungen an den Materialfluss im Unternehmen. Als Folge trat der optimale Ablauf betrieblicher Prozesse wie Disponieren, Lagerung (Just-in-time-Systeme) und deren Kostenminimierung in den Vordergrund des Interesses.

Zweitens fand eine Veränderung der Kostenstruktur statt. Coenenberg (1997, S. 221) verweist auf eine Studie, in welcher der Gemeinkostenanteil an der betrieblichen Wertschöpfung in der amerikanischen Industrie untersucht wurde (Miller \& Vollmann, 1985). Dieser steigt demnach seit 100 Jahren stetig an, während der Anteil der Lohneinzelkosten stark zurückgeht (Miller \& Vollmann, 1985, S. 142). Der hohe Gemeinkostenanteil liegt hauptsächlich in gestiegenen Ausgaben für Forschung und organisatorische Prozesse be- 
gründet. Unter Letztgenannte fassen Coenenberg (1997, S. 221) „die vorbereitenden, planenden, steuernden und überwachenden Tätigkeiten in den Bereich Forschung und Entwicklung, Beschaffung und Logistik, Produktionsplanung und -steuerung, Qualitätssicherung und -prüfung sowie Auftragsabwicklung, Vertrieb und Service“ zusammen. Die bisher genutzten Zuschlagssätze auf die Lohneinzelkosten zur Abdeckung der Gemeinkosten werden dadurch verzerrt. Aus diesem Grund ist es notwendig geworden, die gestiegenen Gemeinkosten verursachungsgerecht zu zerlegen und genauer in das Kostenrechnungssystem zu integrieren.

Der dritte Grund liegt in der Vermeidung von strategischer Fehlsteuerung. Ein Unternehmen steht immer in einem strategischen Verhältnis zu seinen Kunden und seiner Konkurrenz. Um sich als Unternehmen dauerhaft am Markt positionieren zu können, muss das gebotene Preis-Nutzen-Verhältnis besser sein als das der Konkurrenten. Solch ein Wettbewerbsvorteil kann nach Porter (1992, S. 13-20) in zwei Typen unterteilt werden: niedrigere Kosten oder ein Nutzenvorteil, der einen höheren Preis rechtfertigt. Das Unternehmen kann also entweder eine Kostenführer- oder eine Differenzierungsstrategie verfolgen. Beide benötigen jedoch eine genaue Kenntnis der betrieblichen Kosten und der Kostenabhängigkeiten, welche durch klassische Kostenrechnungsmethoden nicht abgedeckt werden. Das Denken in Prozessen hat somit auch Einzug in die betriebswirtschaftliche Kostenrechnung gefunden. Der Aufbau einer Prozesskostenrechnung gliedert sich in verschiedene Teilaufgaben, die sich speziell innerhalb der Prozessbestimmung an der Prozessanalyse aus der Organisationslehre orientieren (Coenenberg, 1997, 225-235; Horváth \& Mayer, 1989, S. 214-219; Horváth \& Renner, 1990, S. 100-103; Jöbstl, 2000, S. 49; Schulte, 2011, S. 138-141):

- Zunächst erfolgt die Prozessbestimmung bzw. die Definition des Hauptprozesses im Unternehmen. Hierzu werden die Prozesse im Sinne der Logistiklehre analysiert und die Prozesskette der Leistungserstellung (Wertschöpfungskette) definiert. Zusätzlich erfolgt die Unterteilung der Prozesskette in Prozesse und einzelne Tätigkeiten (Top-Down-Ansatz).

- Daran anschließend erfolgt die Quantifizierung dieser Aktivitäten. Solche Tätigkeitsanalysen können im Rahmen von Befragungen, Dokumentenanalyse oder durch die Durchführung von Zeitstudien erhoben werden. Dabei werden sowohl die Plan-Prozessmenge als auch die Prozesskosten bemessen. 
- Nun beginnt die Identifikation der Kostentreiber für die leistungsmengeninduzierten Aktivitäten. Durch die Aggregation logisch zusammengehöriger Tätigkeiten zu Prozessen (Bottom-Up-Ansatz), die dem gleichen Kostentreiber unterliegen, ergeben sich kostenstellenübergreifende Hauptprozesse.

- Der letzte analytische Schritt besteht in der Berechnung der Prozesskostensätze. Der Kostentreiber tritt hierbei als erklärende Variable auf. Für die leistungsmengeninduzierten Prozesse lässt sich somit ein Prozess- und ein Hauptprozesskostensatz ermitteln.

Im Anschluss an die Prozesskostenanalyse können die quantifizierten Kostensätze in die Kostenrechnung integriert werden. Dabei treten verschiedene strategische Informationsvorteile auf, die aus der Nutzung solcher Prozesskostensätze resultieren (Coenenberg, 1997, S. 235): Bei stark variablen Einzelkosten kommt es bei der Zuschlagskalkulation zu Verzerrungen infolge der proportionalen Verrechnung der Gemeinkosten (Plötner et al., 2008, S. 130). Durch die Zuordnung der Gemeinkosten über die Inanspruchnahme der Prozesskosten werden die bisher genutzten Zuschlagssätze um einen solchen Allokationseffekt bereinigt. Des Weiteren ermöglicht die Prozesskostenrechnung, die Komplexität und Quantität des Produktsegments als kostenbestimmende Faktoren in die Kostenrechnung zu implementieren. Gegenüber einfachen Produktvarianten haben komplexere Produkte einen deutlich höheren Bedarf an gemeinkostenverursachenden Aktivitäten (Coenenberg, 1997, S. 236). Dieser Komplexitätseffekt wird von der Zuschlagskalkulation vernachlässigt. Abschließend ist der Degressionseffekt zu nennen: Ohne die Prozesskostenrechnung werden die Gemeinkosten als ein konstanter Satz pro Stück verrechnet. Durch eine Erhöhung der Stückzahl folgen die fixen Kostenbestandteile jedoch einem degressiven Zusammenhang. Dieser wird durch die Implementierung der Prozesskostenrechnung in die allgemeine Kostenrechnung sichtbar (Coenenberg, 1997, S. 236238). 


\subsection{Prozesskostenrechnung innerhalb der forstlichen Betriebswirt- schaftslehre}

Die Implementierung der Prozesskostenrechnung in die bisherigen Kostenrechnungssysteme stellt einen vergleichsweisen jungen Zweig des internen Rechnungswesens dar und ist besonders für Unternehmen mit einem hohen Gemeinkostenanteil geeignet. Forstverwaltungen mit einem Gemeinkostenanteil von mehr als $50 \%$ sind demnach prädestiniert, diese Kosten weiter aufzuschlüsseln (Jöbstl, 2000, S. 51). Zur Vereinheitlichung des forstlichen Rechnungswesens wurden 1998 Empfehlungen vom deutschen Forstwirtschaftsrat (DFWR) erarbeitet, die in die Entwicklung des Produktplans FORST mündeten (vgl. Möhring \& Wilhelm, 2012, S. 17). Das forstbetriebliche Leistungsspektrum wird hierzu in fünf Produktbereiche unterteilt, die eine Segmentierung der Verwaltungskosten ermöglichen (DFWR e.V., 2012). Neben der Produktion von Holz und anderen Erzeugnissen wird hier in die Produktbereiche Schutz und Sanierung, Erholung und Umweltbildung, Leistungen für Dritte sowie Hoheits- und sonstige behördliche Aufgaben unterteilt (Möhring \& Wilhelm, 2012, S. 12). Eine weitere Differenzierung der Gemeinkosten kann über eine einfache Kostenstellenrechnung nicht weiter erreicht werden.

Moog (1995, S. 74) schlägt daher vor, die bisher genutzte Form der Deckungsbeitragsrechnung im Forstbetrieb um eine Prozesskostenrechnung zu erweitern bzw. diese zu verbessern, da die Gemeinkosten die Aussagekraft der Deckungsbeitragsrechnung zu stark verringern. Neben Fallstudien wurden bereits erste betriebliche Erfahrungen zur Prozesskostenrechnung im Forstbetrieb gemacht. Im Zuge des Projektes „Neue Betriebsbuchhaltung“ wurde von der Bayerischen Staatsforstverwaltung eine Vorstudie durchgeführt, in der die anfallenden Verwaltungskosten näher analysiert und Prozessen zugeordnet wurden (König \& Schreiber, 1997). Eine Studie von Koepke und Hecker (1998) befasst sich mit der Herleitung von Planprozesskosten für die Unterstützung mittelfristiger Entscheidungen im Forstbetrieb. Hierfür wurde ein privater Forstbetrieb zum Thema Gliederung und Durchführung forstlicher Maßnahmen befragt. Die Fragen reichten von der Räumung von Flächen bis hin zu Durchforstungen von Altbeständen. Als Resultat konnten Prozesskostensätze berechnet und in die Kostenrechnung des Betriebes integriert werden. In beiden Untersuchungen traten jedoch sowohl Erfassungs- als auch Akzeptanzprobleme auf, die eine umfassende Umsetzung der Prozesskostenrechnung behinderten. So stellte auch Jöbstl (2000, S. 52) fest: „Eine permanente Einrichtung der Prozesskostenrechnung 
scheint jedoch auch für Forstbetriebe wenig sinnvoll, vielmehr scheint eine stichprobenartige Verwendung erfolgversprechend.“ Er weist dennoch darauf hin, dass durch die Prozesskostenrechnung ein höheres Gemeinkostenbewusstsein geschaffen wird und einzelne forstliche Tätigkeiten sehr gut untersucht werden können. Sie bietet zusätzlich eine Möglichkeit, die Planungs- und Entscheidungsfindung im Forstbetrieb zu verbessern. Speziell eine Verknüpfung mit einem mathematischen Modell zur Optimierung der Prozesskosten hält Jöbstl (2000, S. 51) für besonders geeignet.

Abseits der forstlichen Betriebswirtschaftslehre ist die Frage nach den prozessabhängigen Ressourcenverbräuchen jedoch sehr viel länger in der Forstwissenschaft existent. Der Schwerpunkt liegt hierbei nicht unmittelbar auf dem Kostenwert an sich, sondern vielmehr auf den Zeitverbräuchen und der Produktivität von forstlichen Tätigkeiten. Beide Forschungsansätze vereint die Frage nach dem prozessabhängigen Verbrauch der begrenzten Ressourcen Zeit und Arbeitskraft. Dieser Forschungsschwerpunkt beschäftigt bereits seit über 90 Jahren die forstliche Arbeitswissenschaft in Form der Leistungsuntersuchung, wie beispielsweise Hilf (1928).

\subsection{Leistungsuntersuchungen innerhalb der forstlichen Arbeitswis- senschaft}

\subsubsection{Stückvolumengesetz}

Begründer der allgemeinen Arbeits- und Leistungsuntersuchung ist der amerikanische Ingenieur F.W. Taylor mit seinem Hauptwerk „The Principles of Scientific Management"“(1911). Er beschreibt darin erstmalig eine wissenschaftliche Auseinandersetzung mit Bewegungs- und Zeitstudien, um Arbeitsprozesse zu analysieren. Hilf (1928; in Speidel, 1984, S. 219) erkannte die Wichtigkeit dieser Forschung, da mit dem Ende des ersten Weltkrieges die Frage der effizienten Gestaltung des nun begrenzten Faktors Arbeitskraft immer wichtiger wurde (Hilf, 1941, S. 8). Bereits 1927 stellten Hilf, Ries und Strehlke fest, dass die Bedeutung des Produktionsfaktors Arbeit in der Forstwirtschaft bis zu diesem Zeitpunkt unterschätzt wurde. Die ersten Bestrebungen dieses neu aufgemachten Forschungsbereiches lagen darin, „die Arbeitskraft zu schonen, (...) seine Leistung richtig zu bemessen und gerecht zu bewerten“ (Hilf, 1941, S. 11). Um solche Erkenntnisse zu 
generieren, wurden Arbeits- und Betriebsuntersuchungen ${ }^{17}$ von forstlichen Tätigkeiten durchgeführt. Innerhalb dieser Versuche wurden einzelne Faktoren wie beispielsweise die Stammstärke manipuliert und ihr Einfluss festgehalten. Dabei beobachtete Hilf (1941) zwei Gesetzmäßigkeiten, die im Rahmen seiner Versuche immer wiederkehrten:

(1) Die Gesetzmäßigkeit der Teilzeiten und

(2) das „Stückmassegesetz“.

Erstgenanntes beruht auf dem Fakt, dass sich alle forstlichen Arbeiten in kleinere Tätigkeiten unterteilen lassen, welche hauptsächlich von wenigen Faktoren abhängig sind. Dadurch können die Teilzeiten im Einzelnen viel genauer beschrieben und erklärt werden als es bei der Gesamtarbeitszeit möglich ist. Die Übergänge dieser Teilzeiten müssen durch klare Schnittpunkte, wie beispielsweise dem Ansetzen der Säge, definiert werden (Hilf, 1941, S. 30). Das Stückmassegesetz wiederum beschreibt die Erkenntnis, dass die Aufarbeitungszeit je Einheit der Holzmasse steigt, je kleiner ein Stück Holz ist. Der Stücklohn [€/Fm] sollte daher im Umkehrschluss mit fallenden Holzstärken ansteigen. Dabei ist anzumerken, dass in Hilfs (1928) Verständnis unter der Masse eines Baumes die Bezugseinheit $\mathrm{m}^{3}$ bzw. Fm gemeint ist. Der Ausdruck Stückvolumengesetz wäre daher deutlich treffender und wird aus diesem Grund auch in der vorliegenden Arbeit so verwendet. ${ }^{18}$ Hilf (1928) begründete die aufgefundenen Zusammenhänge damit, dass Bäume mit geringem Stückvolumen verhältnismäßig viel Nebenarbeit erfordern (z. B. das Vermessen des Baumes). Bei höherem Stückvolumen finden dahingegen hauptsächlich wirksame Arbeiten statt. Durch die Verknüpfung der beiden Gesetzmäßigkeiten kann eine Leistungstafel erstellt werden, welche die Teilzeiten einer forstlichen Tätigkeit in Abhängigkeit von dessen Dimension darstellt (Abbildung 8).

\footnotetext{
${ }^{17}$ Ein Arbeitsversuch untersucht ein Arbeitsverfahren unter kontrollierten Bedingungen (Hilf, 1941, S. 16). Beim Betriebsversuch hingegen wird das Arbeitsverfahren unter realen Bedingungen geprüft und der Einfluss dieser Bedingungen quantifiziert (Hilf, 1941, S. 27). Hilf (1941, S. 28) verwendet hierbei auch synonym den Begriff der Leistungsuntersuchung.

${ }^{18}$ Es bleibt anzumerken, dass im forstlichen Sprachgebrauch noch immer mitunter der Begriff Stückmassegesetz genutzt wird (u. a. Hostettler, 2002, S. 63; Schardt \& Sommer, 2007, S. 23; Stoll et al., 2015, S. 6).
} 


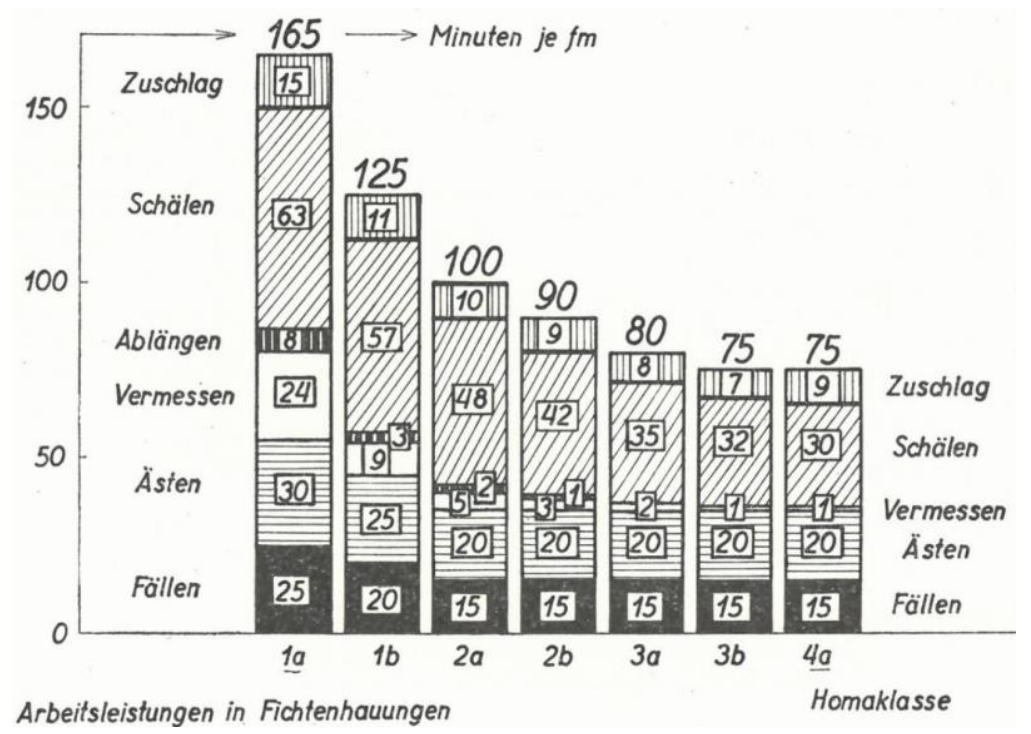

Abbildung 8: Aufarbeitungszeiten je Fm für Fichtenlangholz in Abhängigkeit zum Mittendurchmesser. Abbildung von Hilf (1941, S. 31) auf der Datengrundlage eines Versuches von Hampe im Jahr 1928.

Mathematisch analytisch wurde diese Gesetzmäßigkeit erstmalig von Speidel (1952) im Rahmen seiner Dissertation beschrieben. Als Grundlage dazu dienten die Einheitstarife für Hauerlöhne für die manuelle Holzernte mit der Handsäge. Speidel $(1952$, S. 6) beschreibt das Stückvolumengesetz formal ,als die gesetzmäßige Abhängigkeit der Vorgabezeit von dem Volumen bzw. dem Stück des ausscheidenden Bestandes.“ Er kommt zu dem Schluss, dass die Erlöse eines Durchforstungseingriffes durch die Qualität und die Sortenzusammensetzung bestimmt werden, wohingegen die Kosten hauptsächlich vom mittleren Durchmesser der zu entnehmenden Bäume abhängig sind (Stückvolumen). Speidel (1952) konnte somit mathematisch die theoretischen Annahmen Hilfs (1928) bestätigen, wonach mit Zunahme des Stückvolumens $v[\mathrm{Fm} / \mathrm{Stk}]$ die Bearbeitungszeit (Zeitverbrauch) je Stück $t_{s}[\mathrm{t} / \mathrm{Stk}]$ linear ansteigt (Abbildung 9 und Formel 1). 


$$
t_{s}\left(\frac{t}{S t k}\right)=a\left(\frac{t}{F m}\right) * v\left(\frac{F m}{S t k}\right)+b\left(\frac{t}{S t k}\right)
$$

Formel 1: Lineares Stückvolumengesetz in Anlehnung an Speidel (1952, S. 37).

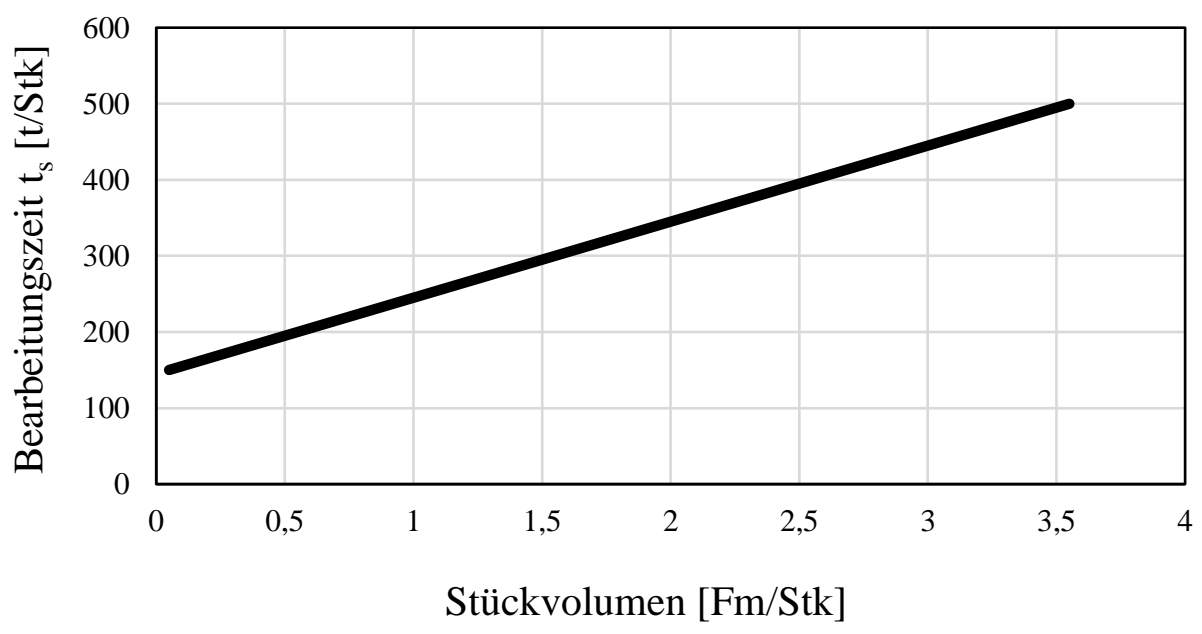

Abbildung 9: Bearbeitungszeit je Stück in Abhängigkeit vom Volumen des ausscheidenden Mittelstammes (Stückvolumengesetz). Eigene Darstellung in Anlehnung an Speidel (1984, S. 219); $t=$ Zeit in Sekunden.

Die Bearbeitungszeit besteht folglich aus den beiden Variablen $a$ und $b$ : Der Faktor $a$ [t/Fm] wird auch als Volumensatz oder Fm-Satz bezeichnet und beschreibt die direkte Abhängigkeit der Bearbeitungszeit vom Stückvolumen. Der Faktor $b$ [t/Stk] gibt wiederum eine konstante Bearbeitungszeit an, die unabhängig vom Volumen des Baumes ist und als Stücksatz definiert wird. Formt man diesen linearen Zusammenhang so um, dass nicht die Bearbeitungszeit je Stück [t/Stk], sondern die Bearbeitungszeit je Volumeneinheit [t/Fm] erklärt wird (Formel 2), gelangt man zu einem hyperbelförmigen Funktionsverlauf (Abbildung 10). ${ }^{19}$

\footnotetext{
${ }^{19}$ Setzt man die Bearbeitungszeit je Fm bzw. Stück in Abhängigkeit vom Stückvolumen des Mittelstammes des ausscheidenden Bestandes, schneiden sich die lineare und Hyperbelfunktion bei einem Stückvolumen von einem Fm (Kleinschmit, 2015, S. 25).
} 


$$
\begin{gathered}
t_{s}\left(\frac{t}{\text { Stk }}\right)=a\left(\frac{t}{F m}\right) * v\left(\frac{F m}{S t k}\right)+b\left(\frac{t}{S t k}\right) \\
t_{v}\left(\frac{t}{F m}\right)=\frac{t_{s}\left(\frac{t}{S t k}\right)}{v\left(\frac{F m}{S t k}\right)} \\
t_{v}\left(\frac{t}{F m}\right)=\frac{a\left(\frac{t}{F m}\right) * v\left(\frac{F m}{S t k}\right)+b\left(\frac{t}{S t k}\right)}{v\left(\frac{F m}{S t k}\right)} \\
t_{v}\left(\frac{t}{F m}\right)=a\left(\frac{t}{F m}\right)+\frac{b\left(\frac{t}{S t k}\right)}{v\left(\frac{F m}{S t k}\right)}
\end{gathered}
$$

Formel 2: Umformung des linearen Stückvolumengesetzes zur Hyperbelform. In Anlehnung an Speidel (1952, S. 37).

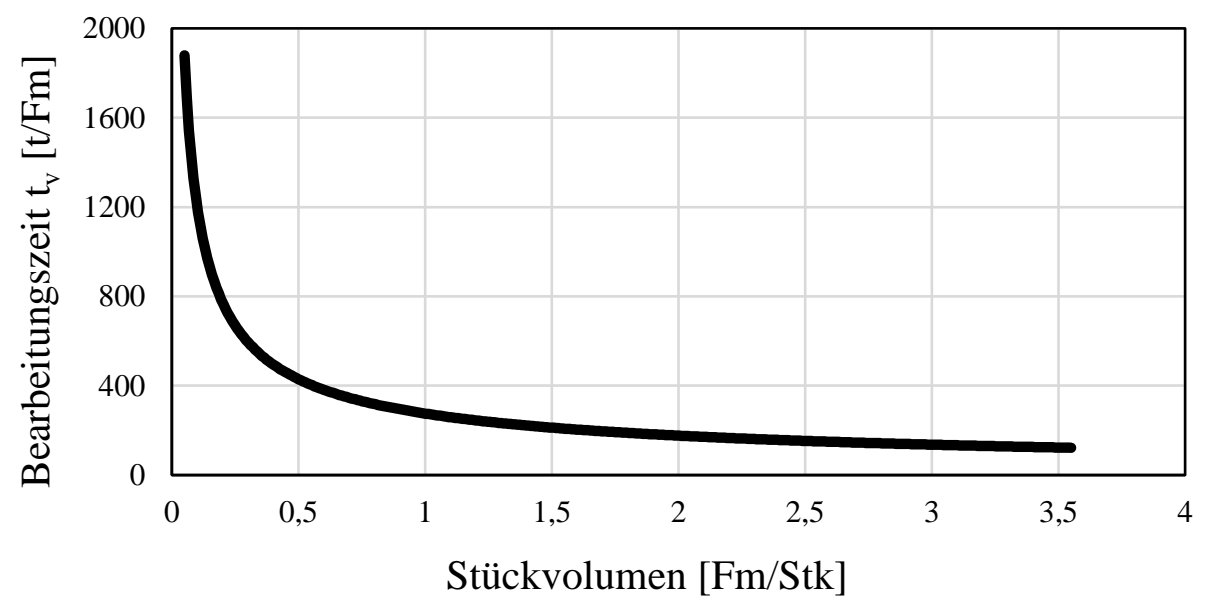

Abbildung 10: Bearbeitungszeit je Festmeter in Abhängigkeit vom Volumen des ausscheidenden Mittelstammes (Stückvolumengesetz). Eigene Darstellung in Anlehnung an Speidel (1984, S. 219); $t=$ Zeit in Sekunden.

Speidel (1952, S. 29) beschreibt den fixen Bestandteil als Nebenzeit, die unabhängig von dessen Volumen bei der Ernte jedes Baumes anfällt. Für die manuelle Holzernte zählt er dazu beispielsweise Tätigkeiten wie „,das Anreichen der Säge, die Zeit bis zum Erfassen der Griffe und die Zeit für das Zielen mit der Säge voraus“ (Speidel, 1952, S. 29).

Das Stückvolumengesetz stellt ein erstes forstliches Modell dar, welches den Zeitverbrauch der manuellen Holzernte in Abhängigkeit zur Dimension der zu bearbeitenden Bäume mathematisch beschreibt. Der grundlegende Einfluss des Stückvolumens auf den 
Zeitverbrauch konnte im Laufe der Jahre auch für die motormanuelle Holzernte (Hegetschweiler, 1988; Pfeiffer et al., 1978), die vollmechanisierte Holzernte (Thor \& Frohm, 1997; Tufts \& Brinker, 1993) sowie weitere forstliche Tätigkeiten nachgewiesen werden (Lüthy, 1997).

\subsubsection{Produktivität}

Die nächsthöhere Aggregationsebene einer forstlichen Leistungsuntersuchung ist die Betrachtung im Kontext der Produktivität ${ }^{20}$, worunter das Verhältnis eines Produktionsoutputs zum notwendigen Produktionsinput verstanden wird (Voigt, Wohltmann et al., 2018, 3. Absatz). Der Begriff der Produktivität lässt sich auch in der betriebswirtschaftlichen Produktions- und speziell in der Kostentheorie wiederfinden. Ziel dieser Theorien ist es, die funktionalen Zusammenhänge zwischen den eigesetzten Produktionsfaktoren und der hergestellten Ausbringungsmenge aufzuzeigen (Wöhe \& Döring, 2010, S. 293). Als Produktionsfaktoren werden alle materiellen und immateriellen Mittel und Leistungen zusammengefasst, die an der Produktion eines Gutes mitwirken. Sie werden klassischerweise in objektbezogene (Elementarfaktoren) und dispositive Faktoren unterteilt (Gutenberg, 1951, S. 3). Zu den Elementarfaktoren gehören die ausführende Arbeit, Betriebsmittel $^{21}$ und Werkstoffe (Betriebsstoffe). Unter die dispositiven Faktoren fällt beispielsweise der notwendige Einsatz für Planung und Leitung des Produktionsprozesses (Wöhe \& Döring, 2010, S. 84).

Ein Produktionsprozess beschreibt damit ein ,technologisch, zeitlich und örtlich bestimmtes effizientes Zusammenwirken von Produktionsfaktoren zur Herstellung einer bestimmten Gütermenge in bestimmter Qualität“ (Voigt, Steven et al., 2018, 2. Absatz). In der Forstwirtschaft wird die Produktivität hauptsächlich beschrieben, indem die mengenmäßige Leistung (meist $\mathrm{m}^{3} \mathrm{bzw}$. Fm der ausgehaltenen Sortimente) ins Verhältnis mit dem dafür notwendigen Arbeitseinsatz (Arbeitszeit, bspw. RAZ des Arbeitsverfahrens) gesetzt wird (REFA - Fachausschuss Forstwirtschaft, 2004, S. 148). Für einen Einzelbaum kann die Produktivität daher wie folgt beschrieben werden:

\footnotetext{
${ }^{20}$ In der Forstwirtschaft auch Arbeitsproduktivität oder Leistung genannt (Purfürst, 2009, S. 21).

${ }^{21} \mathrm{Zu}$ den Betriebsmitteln zählen Maschinen und Werkzeuge, mit denen der Arbeiter den Systeminput zur Erledigung der Arbeitsaufgabe bearbeitet (Jaeger, 2020, 4. Folie).
} 


$$
\text { Produktivität Einzelbaum }\left[\frac{m^{3}}{R A Z}\right]=\left(\frac{\text { geerntetes Holz Einzelbaum }\left[m^{3}\right]}{\text { Zeitverbrauch Einzelbaum }[R A Z]}\right)
$$

Formel 3: Produktivität Einzelbaum in Anlehnung an Purfürst (2009, S. 22).

Durch die Annahme, dass sich ein Bestand aus der Summe der darin existierenden Einzelbäume zusammensetzt, kann die Produktivität auf Bestandesebene durch die folgende Funktion beschrieben werden:

$$
\text { Produktivität Bestand }\left[\frac{m^{3}}{R A Z}\right]=\sum_{i=1}^{N_{B}} \text { Produktivität Einzelbaum }{ }_{i}\left[\frac{m^{3}}{R A Z}\right]
$$

Formel 4: Produktivität Bestand; $N_{B}=$ Anzahl Einzelbäume im Bestand.

Da sich diese Arbeit mit der Erstellung eines solchen Produktivitätsmodells für die mechanisierte Holzaufarbeitung bei der Buche beschäftigt, ist es notwendig, die bestehenden Produktivitätsmodelle zu systematisieren. Diese unterscheiden sich vor allem darin, wie der Einfluss des Baumvolumens auf den Verlauf der Produktivität ausfällt (Purfürst, 2009, S. 87). Aus diesem Grund gibt es eine Vielzahl an unterschiedlichen Produktivitätsmodellen, die sich in der Transformation der unabhängigen Variable Baumvolumen ${ }^{22}$ unterscheiden (Tabelle 1).

Tabelle 1: Übersicht über bestehende Produktivitätsmodelle, unterteilt nach der Transformation der unabhängigen Variablen: Baumvolumen.

\begin{tabular}{ll}
\hline Studie & Produktivitätsmodell \\
\hline Pausch (2002); Labelle und Huss (2018) & Quadratisch \\
Alam et al. (2014); Krell (2006) & Logarithmisch \\
Häberle (1984); Heinimann (2001); & Potenzfunktion \\
Labelle et al. (2016); Strandgard und Mitchell (2020) & \\
Lange (2006); Stampfer und Steinmüller (2004) & Reziproke-Hyperbel $^{23}$ \\
\hline
\end{tabular}

\footnotetext{
${ }^{22}$ Das in den Studien aufgeführte Baumvolumen wird nach Waldmaß der verkaufsfähigen Masse berechnet und stellt somit das reelle Erntevolumen (Efm) dar (vgl. Purfürst, 2009, S. 73).

${ }^{23}$ Hyperbel für die Zeitverbrauchswerte je Fm werden in beiden Studien über folgendes Modell gebildet (Purfürst, 2009, S. 87): $y=a *$ Baumvolumen $^{b}+c$.
} 
Ein wichtiger Aspekt, den nur wenige Produktivitätsmodelle für die mechanisierte Holzaufarbeitung berücksichtigen, ist die negative Grenzleistung über dem Baumvolumen (Purfürst, 2009, S. 88). Rein praktisch betrachtet nimmt die Produktivität - im Sinne des klassischen Stückvolumengesetzes - bei steigendem Baumvolumen zunächst zu, sinkt jedoch ab einem Punkt der maximalen Leistung wieder ab. Die Gründe hierfür können in der Grenzleistungsfähigkeit der Betriebsmittel (Harvester und Aggregat) und einem erschwerten Handling bei stärker dimensionierten Bäumen liegen (Kärhä et al., 2004, S. 53-54). Demnach nimmt auch die Produktivität eines Harvesters ab einem bestimmten Baumvolumen ${ }^{24}$ wieder ab (Nurminen et al., 2006, S. 336), was einer negativen Grenzproduktivität entspricht. Dies würde bedeuten, dass bereits im „klassischen“ Stückvolumengesetz der lineare Verlauf der Bearbeitungszeit pro Baum in Abhängigkeit zum mittleren Baumvolumen des ausscheidenden Bestandes einem nichtlinearen Zusammenhang folgen müsste. Ist solch eine Relation nicht in den Daten erkennbar, kann abgeleitet werden, dass im Rahmen der untersuchten Baumdimensionen keine Leistungsgrenze erreicht wurde. Aus diesem Grund können hier auch diejenigen Modelle berücksichtigt werden, die keine negative Grenzleistung implementieren. Purfürst (2009, S. 88) empfiehlt jedoch, für solche Modelle einen Gültigkeitsbereich festzulegen.

Als grundlegende Vorgehensweise wird in dieser Arbeit der Ansatz verfolgt, dass nicht die Produktivitätsfunktion direkt über eines der Produktivitätsmodelle aus Tabelle $1 \mathrm{ab}$ geleitet wird, sondern der Zeitverbrauch am Einzelbaum linear erklärt und im Rahmen der vorgestellten Umformungen zunächst zu einer Hyperbel (Formel 2) und im Anschluss zu einer Reziproken-Hyperbel umgestellt wird (Formel 5). Dies geschieht, um eine nicht notwendige Transformierung der abhängigen Variablen zu vermeiden. Daraus folgt eine Produktivitätsfunktion, die zunächst einen überproportionalen Anstieg beschreibt und sich dann mit steigendem Stückvolumen asymptotisch einer Maximalleistung annähert (Abbildung 11).

\footnotetext{
${ }^{24}$ Da es sich hier um den Punkt der höchsten Produktivität handelt, wird dieser im Englischen auch sweetspot genannt (Alam et al., 2014, S. 14).
} 


$$
\begin{gathered}
t_{v}\left(\frac{t}{F m}\right)=a\left(\frac{t}{F m}\right)+\frac{b\left(\frac{t}{S t k}\right)}{v\left(\frac{F m}{t}\right)} \\
p_{v}\left(\frac{F m}{t}\right)=\frac{1}{t_{v}\left(\frac{t}{F m}\right)} \\
p_{v}\left(\frac{F m}{t}\right)=\frac{1}{a\left(\frac{t}{F m}\right)+\frac{b\left(\frac{t}{S t k}\right)}{v\left(\frac{F m}{S t k}\right)}} \\
p_{v}\left(\frac{F m}{t}\right)=\frac{v\left(\frac{F m}{S t k}\right)}{a\left(\frac{t}{F m}\right) * v\left(\frac{F m}{S t k}\right)+b\left(\frac{t}{S t k}\right)}
\end{gathered}
$$

Formel 5: Umformung des „,klassischen“ Stückvolumengesetzes im Rahmen einer Reziprok-Hyperbel-Umformung.

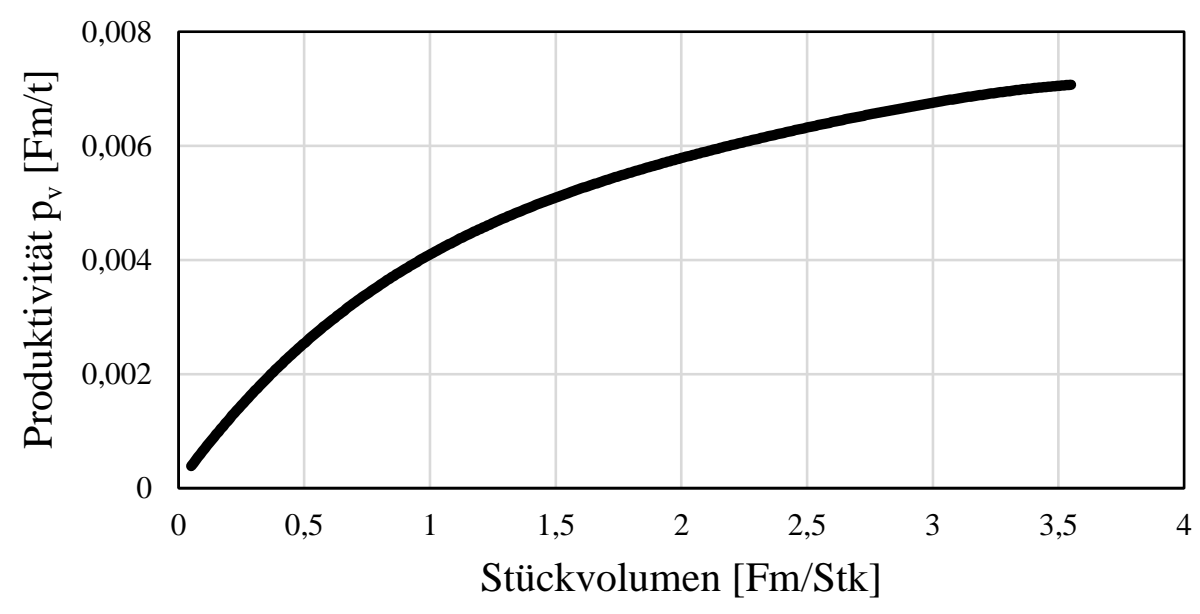

Abbildung 11: Produktivität in Abhängigkeit vom Volumen des ausscheidenden Mittelstammes (Stückvolumengesetz, eigene Darstellung); $t=$ Zeit in Sekunden.

Durch die Anpassung der Grundgleichung für die Bearbeitungszeit je Stück kann die negative Grenzproduktivität in die Formel integriert werden $\left(t_{s}\right.$, Formel 1). Anstelle eines linearen Verlaufes weist die Funktion einen überproportionalen Anstieg auf, der im Anschluss in eine Reziproke-Hyperbel mit negativer Grenzproduktivität umgeformt wird. Zur Überprüfung dieser Methodik wird in Formel 8 beispielhaft die Umformung einer quadratischen Grundgleichung zur Beschreibung der Bearbeitungszeit je Stück abgebildet. 


$$
\begin{gathered}
t_{s}\left(\frac{t}{S t k}\right)=a_{1}\left(\frac{t}{F m}\right) * v\left(\frac{F m}{S t k}\right)+a_{2}\left(\frac{t}{F m}\right) * v^{2}\left(\frac{F m^{2}}{S t k}\right)+b\left(\frac{t}{S t k}\right) \\
t_{v}\left(\frac{t}{F m}\right)=\frac{t_{s}\left(\frac{t}{S t k}\right)}{v\left(\frac{F m}{S t k}\right)} \\
t_{v}\left(\frac{t}{F m}\right)=a_{1}\left(\frac{t}{F m}\right)+a_{2}\left(\frac{t}{F m}\right) * v\left(\frac{F m}{S t k}\right)+\frac{b\left(\frac{t}{S t k}\right)}{v\left(\frac{F m}{S t k}\right)} \\
p_{v}\left(\frac{F m}{t}\right)=\frac{1}{t_{v}\left(\frac{t}{F m}\right)} \\
p_{v}\left(\frac{F m}{t}\right)=\frac{v\left(\frac{F m}{S t k}\right)}{a\left(\frac{t}{F m}\right) * v\left(\frac{F m}{S t k}\right)+a_{2}\left(\frac{t}{F m}\right) * v^{2}\left(\frac{F m^{2}}{S t k}\right)+b\left(\frac{t}{S t k}\right)}
\end{gathered}
$$

Formel 6: Umformung des quadratischen Stückvolumengesetzes zur Hyperbelform. Grau hinterlegt ist der quadratische Funktionsterm.

\subsubsection{Leistungsbeeinflussende Faktoren}

Wie Hilf (1928) bereits erkannte, ist das Volumen der zu entnehmenden Bäume der Haupteinflussfaktor des notwendigen Zeitverbrauchs bei vielen forstlichen Prozessen. Neben dem Volumen der zu erntenden Bäume sind allerdings auch weitere Faktoren des forstlichen Arbeitsumfeldes von Heterogenität geprägt und können die Produktivität beeinflussen (Purfürst, 2009, S. 27). Welche Faktoren Einfluss nehmen, wurde in vielen Versuchen erforscht und in der Literatur beschrieben (Kapitel 4.4). So kann beispielweise eine steigende Geländeneigung die Produktivität eines Harvesters verringern (Kellogg \& Bettinger, 1994; Schöttle et al., 1997). Die allgemeine Arbeitssystemdarstellung des REFA-Fachausschusses Forstwirtschaft (1998, S. 4) ermöglicht eine Klassifizierung dieser leistungsbeeinflussenden Faktoren (sog. Kostentreiber). 
Tabelle 2: Übersicht über die leistungsbeeinflussenden Faktoren nach REFA-Fachausschuss Forstwirtschaft (1998, S. 40-44).

\begin{tabular}{ll}
\hline Faktoren & Beispiele \\
\hline Input & Baumart, Baumvolumen \\
Umweltbedingungen & Bestandesdichte, Geländeneigung \\
Mensch & Erfahrung, Eignung, Leistungsniveau über den Tag \\
Arbeitsmittel & Maschinentyp, Ergonomie \\
Arbeitsaufgabe & Restriktionen, Eingriffsstärke, Bestandesvorbereitung \\
Output & Sortimente, Sortimentsvielfalt \\
\hline
\end{tabular}

Durch die Einbeziehung dieser Faktoren in den Versuchsaufbau einer Studie kann die Einflussstärke in die entstehenden Produktivitätsmodelle als unabhängige Variablen integriert werden. Die Faktoren können sowohl einen voneinander unabhängigen als auch stark wechselseitigen Charakter aufweisen. So bestimmt beispielweise die Baumart sowie das Baumvolumen maßgeblich über die mögliche Auswahl an Sortimenten bzw. die Sortimentsvielfalt. Mit jedem einzubeziehenden Faktor steigt die notwendige Datengrundlage daher gravierend an.

Eine große Herausforderung bei der Erstellung forstlicher Produktivitätsmodelle besteht zusätzlich im erheblichen Aufwand und den fehlenden Laborbedingungen (Alam et al., 2014, S. 21). Aufgrund verschiedener Forschungsansätze und Datengrundlagen unterscheiden sich die entwickelten Modelle sehr stark voneinander (Purfürst, 2009, S. 24-26). Sie reichen von einfachen Studien, in denen die einzige erklärende Variable das Stückvolumen darstellt, bis hin zu der Entwicklung komplexer Modelle, in der eine Vielzahl an Kovariablen integriert wurden. Für eine ausführliche Auseinandersetzung mit den bisher entwickelten Produktivitätsmodellen im Bereich der Forstwirtschaft wird an dieser Stelle auf die Arbeit von Cremer (2008) verwiesen, der sich meta-analytisch der Thematik angenähert hat. Neben der Betrachtung aus einem arbeitswissenschaftlichen Kontext wurden zudem erste wissenschaftliche Studien zur Überführung forstlicher Produktivitätsmodelle in die Prozesskostenrechnung durchgeführt (Nurminen et al., 2009). Eine weitergehende Auseinandersetzung mit dem bestehenden Forschungsstand zum Thema Produktivitätsmodelle für die mechanisierte Holzaufarbeitung findet in Kapitel 4.4 statt. Um die Datengrundlage für die Erstellung eines Produktivitätsmodells zu schaffen, kommen hierbei hauptsächlich Zeitstudien zum Einsatz. 


\subsection{Forstliche Arbeitszeitstudien}

Zeitstudien ermöglichen die Quantifizierung von Produktivitätsmodellen bzw. die Durchführung von Leistungsuntersuchungen. Ihre forstliche Bedeutung erhielten sie insbesondere in der Herleitung von Vorgabezeiten für die motormanuellen Holzerntetarife (HET). Auch die in Kapitel 3.3 vorgestellten Arbeiten beruhen auf dieser Methodik. Eine Zeitstudie verfolgt nach Löffler (1992, S. 214) die folgenden Ziele:

- Messung der zur Erledigung eines Arbeitsauftrages benötigten Gesamtarbeitszeit (GAZ) sowie die Anteile der einzelnen Tätigkeiten bzw. Ablaufabschnittszeiten

- Registrierung der im Arbeitsverlauf geleisteten Ergebnisse und Festlegung der Bezugsmengen

- Erfassung des Zeitbedarfes und die Auswirkungen von beeinflussenden Faktoren (Kostentreiber)

Die Gesamtarbeitszeit (GAZ) setzt sich aus der reinen Arbeitszeit (RAZ) und den allgemeinen Zeiten (AZ) zusammen. Zu den allgemeinen Zeiten gehören die persönlichen Verteilzeiten für Pausen oder Telefonate und die sachlichen Verteilzeiten für Wartung, Reparaturen und Umsetzen (von Bodelschwingh, 2006, S. 29).

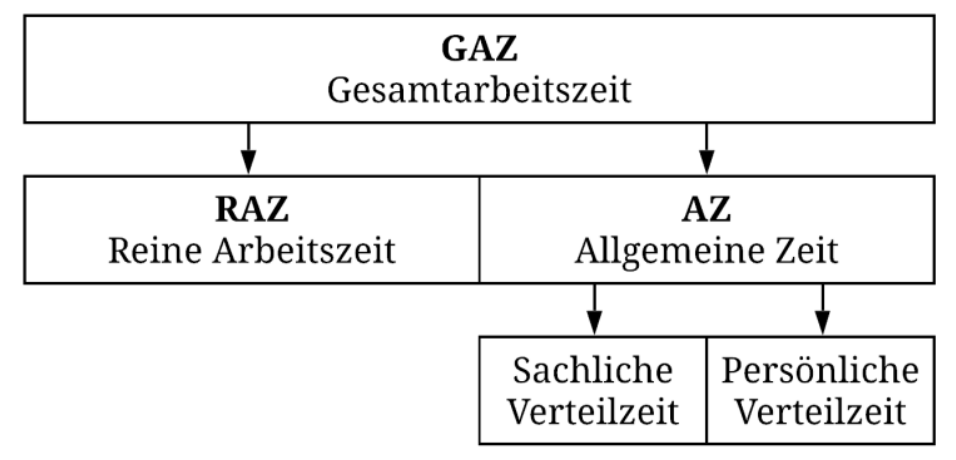

Abbildung 12: Differenzierung der Arbeitszeiten (eigene Darstellung in Anlehnung an von Bodelschwingh, 2006, S. 29).

Bei der Untersuchung von maschinellen Arbeitsmitteln wird häufig auch der Begriff der MAS (Maschinenarbeitsstunde) genutzt. Damit ist die Zeit gemeint, in der die Maschine produktiv arbeitet. Es wird hierbei zwischen der $\mathrm{MAS}_{15}$, die Unterbrechungen von weni-

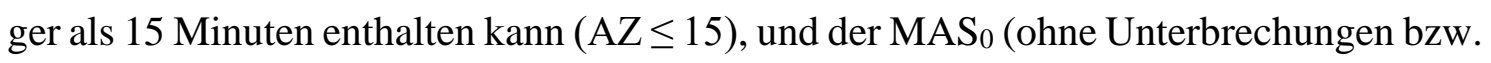
RAZ) unterschieden (Purfürst, 2009, S. 234). 
In der vorliegenden Arbeit wird nur die $\mathrm{MAS}_{0}$ der Harvestersysteme untersucht. Bereits Speidel (1952, S. 29-30) weist darauf hin, dass sich der Zeitverbrauch bei der Holzernte in weitere fixe und variable Komponenten zerlegen lässt, die bisherigen forstlichen Zeitstudien aber keine ausreichende Datengrundlage dafür bieten. Aufgrund der teilweise sehr kurzen Dauer einzelner Ablaufabschnitte und der Überlappung von Tätigkeiten wird im Bereich forstlicher Zeitstudien allgemein das Fortschrittszeitverfahren angewandt (von Bodelschwingh, 2006, S. 30). Dabei werden die Zeitverbräuche der Ablaufabschnitte von Anfang bis Ende chronologisch entlang ihrer Entstehung gemessen. Grundlage hierfür sind im Vorfeld definierte Trennpunkte, die den Anfang und das Ende einer Tätigkeit beschreiben. Durch das Aggregieren der Ablaufabschnitte lässt sich der Arbeitsprozess wieder zusammensetzen und die Dauer eines Arbeitszyklus berechnen. Als Standard zur Durchführung einer Zeitstudie etablierte sich im deutschsprachigen Raum die Anweisungsausführungen des REFA e.V. (vgl. Purfürst, 2009, S. 5). Dies wird auch in Veröffentlichungen sichtbar, in denen das Verfahren als „Zeitstudie nach REFA“ beschrieben wird (Schlick et al., 2010, S. 672).

Als weitere Datenquellen kommen mitunter Eigenaufzeichnungen, speziell der Forstmaschinenführer, zum Einsatz. Durch die Weiterentwicklung der Holzerntetechnologie werden dabei Parameter wie die Art, der Mittendurchmesser und die Länge eines Sortimentes gespeichert und als Daten exportiert. Speziell die Verbindung von Eigenaufzeichnungen mit den Ergebnissen der Zeitstudien kann einen methodischen Mehrgewinn bieten.

\subsection{Kapitelzusammenfassung}

Dieses Kapitel beschäftigte sich mit der Prozesskostenrechnung im Allgemeinen sowie im forstwirtschaftlichen Kontext. Dazu erfolgte im ersten Schritt eine Einordnung in das allgemeine System der betriebswirtschaftlichen Kostenrechnung und die historische Entwicklung der Prozesskostenrechnung. Daran anknüpfend wurde die prozessorientierte Kostenanalyse im forstwirtschaftlichen Bezugsrahmen näher beleuchtet. Es konnte gezeigt werden, dass die Frage nach den prozessabhängigen Ressourcenverbräuchen die forstliche Arbeitslehre schon seit geraumer Zeit - konkret seit rund 90 Jahren - bewegt. Ein besonderer Fokus liegt dabei auf Produktivitätsmodellen und dem Einfluss des Stückvolumens eines Baumes als Kostentreiber. Da auch weitere Faktoren des forstlichen Ar- 
beitsumfeldes von Heterogenität geprägt sind, können diese ebenfalls die Leistung beeinflussen. Dieser Zusammenhang ist besonders bei der Planung und Erstellung eines Produktivitätsmodells wichtig. Je mehr unabhängige Variablen in das Modell eingehen, desto größer wird die Genauigkeit - aber gleichzeitig auch die Komplexität. Grundlage solcher Modelle sind klassischerweise forstliche Arbeitszeitstudien.

Zur Erstellung eines forstlichen Produktivitätsmodells für die mechanisierte Buchenaufarbeitung ist die Prüfung des Forschungsstandes ein notwendiger Schritt. Das nächste Kapitel beschreibt daher die Grundlagen und Rahmenbedingungen der technischen Produktion von Buchenrohholz. 


\section{Stand, Entwicklung und Herausforderungen der mecha- nisierten Aufarbeitung von Buchenrohholz}

In Kapitel 4 erfolgt zunächst eine Betrachtung der morphologischen Besonderheiten der Buche und den daraus resultierenden Eigenschaften für das Produkt Rohholz. Im Anschluss folgt ein Blick auf die historischen Aufarbeitungsverfahren des Bucheneinschlags. Diese wird ergänzt durch eine Darstellung der Ursachen, die den wachsenden Einsatz von Harvestern vorantreiben und auch den Problemen, die daraus resultieren. Abschließend wird der Forschungsstand zu Produktivitätsmodellen der mechanisierten Holzaufarbeitung beleuchtet und dabei die wissenschaftliche Lücke aufgezeigt, die mit dieser Arbeit geschlossen werden soll (Abbildung 13).

\begin{tabular}{|l|}
\hline $\begin{array}{c}\text { Kapitel 4: Stand, Entwicklung und Herausforderungen der } \\
\text { mechanisierten Aufarbeitung von Buchenrohholz }\end{array}$ \\
\hline $\begin{array}{l}\text { 4.1 Wuchsdynamik und Kronentypisierung } \\
\text { Morphologische Eigenschaften, Kategorisierung von } \\
\text { Kronentypen, weitere Qualitätsmerkmale }\end{array}$ \\
\begin{tabular}{|l|} 
4.2 Produkt Buchenrohholz \\
Produktionszusammenhänge, Sortierung, Voraussetzungen als \\
technischer Rohstoff
\end{tabular} \\
$\begin{array}{l}\text { 4.3 Arbeitsverfahren der Buchenholzernte } \\
\text { Historie der Buchenholzernte, Verfahrensentwicklung, technische } \\
\text { Bedingungen für den Harvestereinsatz in Buchenbeständen }\end{array}$ \\
$\begin{array}{l}\text { 4.4 Produktivitätsmodelle der mechanisierten Holzaufarbeitung } \\
\text { Forschungsstand: Produktivitätsmodelle im Allgemeinen, bisherige } \\
\text { Studien zum Harvestereinsatz in Laubholzbeständen }\end{array}$ \\
\hline
\end{tabular}

Abbildung 13: Thematische Schwerpunkte im Kapitel 4 - Stand, Entwicklung und Herausforderungen der mechanisierten Aufarbeitung von Buchenrohholz. 


\subsection{Wuchsdynamik und Kronentypisierung}

Um morphologische Größen und deren Einfluss auf die Aufarbeitungsproduktivität verstehen zu können, bedarf es eines botanischen Grundlagenwissens der untersuchten Baumart. Die Buche (= Rotbuche, Fagus sylvatica L.) gehört zu den Klimaxbaumarten Mitteleuropas (Röhrig et al., 2006, S. 49). Aufgrund ihrer großen Schattentoleranz und der hohen Intensität ihres Bestandesschattens verfügt sie über eine starke Konkurrenzkraft. Unabhängig vom Säuregehalt und Humuszustand des Bodens zeichnet sich die Rotbuche durch eine hohe Standortsamplitude aus und kommt somit auf fast allen Bodentypen vor. Abgesehen von den höheren Lagen in den Alpen und von deren niederschlagsarmen und winterkalten inneren Tälern ist die Rotbuche in Mitteleuropa unter natürlichen Verhältnissen beinahe allgegenwärtig (Ellenberg \& Dierschke, 2010, S. 149).

Wie bei anderen Baumarten auch, ist das Verzweigungssystem der Buche genetisch determiniert (sog. angeborenes System, Hallé et al., 1978, S. 74). Die Buchentriebe wachsen waagerecht (plagiotrop) - mit Ausnahme des Terminaltriebs, welcher sich verspätet aufrichtet. Dieser wächst jährlich aus der gleichen Terminalknospe. Damit gehört die Buche zu den monopodial verzweigenden Baumarten (Husmann, 2013, S. 3). Der typische stockwerkartige Aufbau der Buche ist eine Folge der nach jetzigem Stand der Wissenschaft genetisch determinierten Apikaldominanz der Terminalknospe, wegen der die Seitentrieblängen mit steigendem Abstand zur Terminalknospe zunehmen (sog. Akrotonie; Kleinschmit, 2015, S. 11). Die Länge der Triebe wird zusätzlich durch Lichtgenuss und Alter beeinflusst. Sie differenzieren sich in Langtriebe - zuständig für die Annexion des Luftraums - und Kurztriebe, welche die Hauptaufgabe der Assimilation bewältigen. Speziell bei jungen Bäumen entwickeln sich bei stärkerem Lichtgenuss verstärkt Langtriebe. Bei der natürlichen Astreinigung sterben zuerst die Kurztriebe und nachfolgend die Langtriebe ab (Roloff, 1985, S. 84-86).

Eine weitere Besonderheit der Buche ist die Differenzierung des Astablaufwinkels, also dem Winkel zwischen der Stammachse und dem Seitentrieb. Hengst (1964, S. 79) beschreibt den Astablaufwinkel als einen Weiser für die soziologische Stellung des Einzelbaumes im Bestand, der zusätzlich erb- und umweltbedingten Schwankungen unterliegt. Nach Roloff (1985, S. 56) ist der Astablaufwinkel bei Schattbuchen größer als bei Lichtbuchen. Zusätzlich vergrößert sich dieser gewichtsbedingt mit dem Alter des Zweiges dieser kann von $40^{\circ}$ bei tief angesetzten Ästen auf bis zu $90^{\circ}$ bei Altbuchen ansteigen 
Die mechanisierte Aufarbeitung von Buchenrohholz

(Roloff, 1985, S. 56). Mit voranschreitendem Baumalter wird das Verzweigungssystem der Buche durch eine Reihe von Umweltfaktoren in seiner Entwicklung beeinflusst und wechselt von einem angeborenen, akrotonischen Modell zu einem opportunistischen Modell (Hallé et al., 1978, S. 269). Die größten Veränderungen werden dabei durch den Verlust oder eine Verletzung der Terminalknospe verursacht. Im Extremfall entsteht eine zwieselförmige Verzweigung, bei der zwei Sprossen gleichermaßen dominant sind. Je nach Schaftachse und Astablaufwinkels kann die Ausbildung der Krone nach Hengst (1964, S. 82) in drei Hauptkronentypen ${ }^{25}$ eingeteilt werden (Abbildung 14):
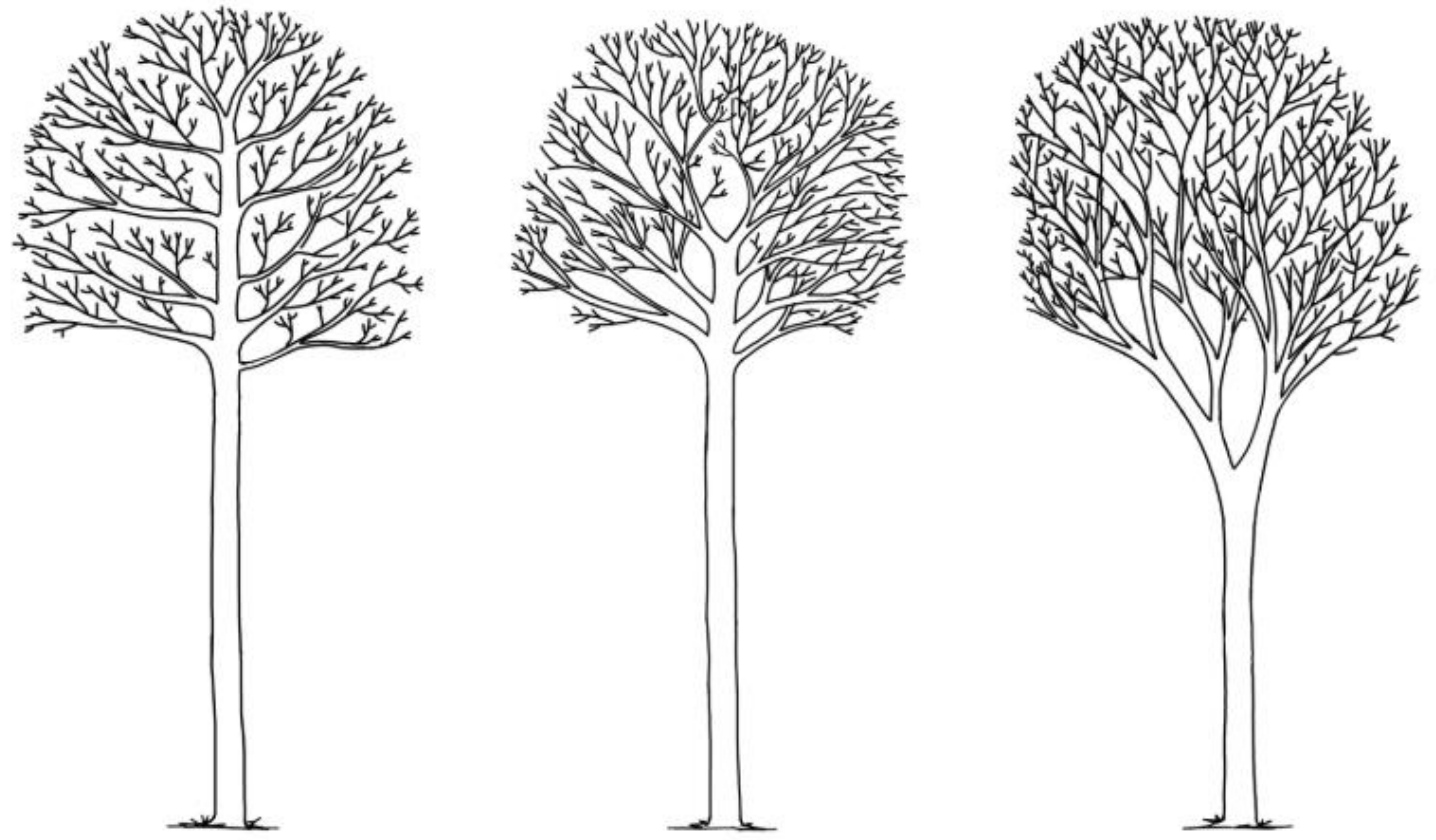

Abbildung 14: Wipfelschäftiger (links), besenförmiger (mittig) und zwieselförmiger (rechts) Kronentyp bei der Buche (Quelle: Kleinschmit, 2015, S. 13, gespiegelt).

Zum wipfelschäftigen Typus werden Bäume gezählt, deren Schaftachse durchgehend ist und deren Astablaufwinkel sich in einem Bereich zwischen $65^{\circ}$ und $90^{\circ}$ bewegen. Nach Kleinschmit (2015, S. 12) ,entsprechen sie damit am ehesten dem forstlichen Ausleseziel mit dem vornehmlichen Ziel der Wertholz- bzw. Stammholzproduktion.“ Bäume des besenförmigen Kronentyps zeichnen sich zum einen durch steilere Astablaufwinkel (zwischen $65^{\circ}$ und $75^{\circ}$ ) aus. Zum anderen besitzen sie eine Schaftachse, die sich im oberen

\footnotetext{
${ }^{25}$ Vernachlässigt wird hier der Bukettkronentyp, der als Sonderform des wipfelschäftigen Kronentyps verstanden werden kann. Dieser unterscheidet sich dadurch, dass sich die Schaftachse im oberen Fünftel der Krone fächerförmig auflöst (Hengst, 1964, S. 79).
} 
Viertel der Baumhöhe in Haupt- und Nebenäste zu einer charakteristischen besenartigen Form auflöst. Zum zwieselförmigen Kronentypus zählen Bäume, deren Schaftachse sich deutlich unterhalb des oberen Viertels auflöst. Aufgrund dieser tiefen Teilung bildet der Baum eine deutlich voluminösere Krone aus und steht mit seiner kurzen Schaftlänge dem forstlichen Produktionsziel entgegen (Kleinschmit, 2015, S. 12). Des Weiteren zeichnet sich der zwieselförmige Kronentyp durch deutlich steilere Astablaufwinkel von bis zu $10^{\circ}$ aus (Hengst, 1964, S. 79-81; Richter, 1990, S. 12).

Die Systematik nach Hengst konnte in zahlreichen Studien beschrieben oder bestätigt werden (u. a. von Arnswald, 1951, S. 174; Kleinschmit, 2015, S. 11-14; Krahl-Urban, 1953, S. 246, 1955, S. 402, 1962, S. 29; Roloff, 1988, S. 297; Schmidt, 2001, S. 110). Gemäß Hengst (1964, S. 80) ist der besenförmige Kronentyp die am häufigsten in der Natur ausgebildete Form, gefolgt vom zwieselförmigen Typus. Am seltensten ist der wirtschaftlich attraktive wipfelschäftige Kronentyp.

Wie bereits beschrieben ist die Form und Höhe der Auflösung der Schaftachse besonders ausschlaggebend für die Zuordnung zu einer der drei Kategorien. Hierbei stellt sich grundsätzlich die Frage, ob es sich bei der Verzweigung entweder um einen wirklichen Zwiesel, also die Auflösung der Schaftachse in zwei oder mehrere Hauptäste, handelt oder aber um die Ausprägung eines Steilastes, welcher nicht unbedingt die Auflösung der Schaftachse nach sich zieht (Kleinschmit, 2015, S. 13-14). Diese Unterscheidung ist stark definitionsabhängig und wird in der bestehenden Literatur sehr differenziert vorgenommen (vgl. Börner et al., 2003, S. 276; Richter, 1990, S. 12; S. Rumpf et al., 2011, S. 35). Als wichtiges Maß hat sich aber das Verhältnis zwischen dem Durchmesser des Hauptschaftes und dem Durchmesser des verzweigenden Astes durchgesetzt. Je nach Definition spricht man von einem Zwiesel, wenn der Durchmesser des konkurrierenden Astes an seiner Basis mindestens 75 \% (Börner et al., 2003, S. 276) bis 80 \% (Richter, 1990, S. 12) des Durchmessers des Hauptastes aufweist.

Eine ähnliche Uneinigkeit herrscht über den genauen Punkt des Kronenansatzes bei der Buche bzw. bezüglich der Frage, ob der Punkt der Auflösung der Schaftachse als Kronenansatzhöhe definiert werden kann (Rumpf et al., 2011, S. 34; von Wilpert et al., 2011, S. 12). Im forstlichen Versuchswesen hat sich die Höhe des ersten grünen Primärastes, unabhängig von dessen Stärke bzw. dem Durchmesserverhältnis zur Schaftachse, als Kronenansatzhöhe etabliert (Dieler \& Pretzsch, 2013, S. 100; Hein, 2007, S. 18-19; Schmidt, 2001, S. 19). 
Die mechanisierte Aufarbeitung von Buchenrohholz

Für eine umfassende morphologischen Bewertung von Einzelbäumen werden neben dem Kronentyp auch weitere Qualitätsmerkmale benötigt (Gadow, 2003, S. 79). Daher ist es hilfreich, Kriterien der Holzqualität mit Hilfe von Variablen zu beschrieben, die durch die Wachstumsbedingungen beeinflusst werden. Viele dieser Merkmale stehen in direkter Verbindung mit dem Produkt Rohholz und dessen Gütestufen, auf die daher nachfolgend näher eingegangen werden soll.

\subsection{Produkt Buchenrohholz}

„Unter Rohholz versteht man gefällte, entwipfelte und/oder entastete Bäume sowie Baumteile“ (DFWR e.V. \& DHWR e.V., 2020, S. 2). ${ }^{26}$ Die Aufarbeitung des Baumes ermöglicht die Zerlegung des Stammes in Abschnitte, die nach sortenspezifischen Anforderungen eingeteilt werden können (Kleinschmit, 2015, S. 25). Die Anforderungen an das Produkt Rohholz verändern sich ständig und folgen den Veränderungen bzw. Entwicklungen der Holwirtschaft (Schulz et al., 1988, S. 317).

Ein wichtiger Baustein für den Handel mit Rohholz ist die Einteilung des Rohstoffes in verschiedene Sorten, die spezifische Anforderungen erfüllen. Die privatrechtliche Rahmenvereinbarung für den Rohholzhandel (RVR), die zwischen dem Deutschen Forstwirtschaftsrat e.V. und dem Deutschen Holzwirtschaftsrat e. V. besteht, bildet seit ihrer Schließung im Jahr 2014 einen bundeseinheitlichen Standard für die Sortierung von Rohholzabschnitten (DFWR e.V. \& DHWR e.V., 2020). ${ }^{27}$ Die für diese Arbeit entscheidenden Inhalte dieser Vereinbarung sollen folgend kurz vorgestellt werden:

Die grundlegende Einteilung wird bestimmt durch die weitere wirtschaftliche Verwendung der Abschnitte und ist unterteilt in drei Sortimente: Stamm-, Industrie- und Energieholz. Zusätzlich können Sondersortimente ausgehalten werden, die für eine spezifische Verwendung vorgesehen sind und daher bestimmte Kriterien erfüllen müssen. Dazu zählt beispielsweise eine gesonderte Aushaltung von Abschnitten für die Produktion von Paletten, Schwellen oder auch Masten.

\footnotetext{
${ }^{26}$ Oft synonym zum Begriff des Rohholzes wird der Begriff Rundholz verwendet. Dieser umfasst aber nur den runden und ungeteilten Teil des Rohholzes. Daneben definiert Rohholz sowohl gehacktes als auch aufgespaltenes Holz (DFWR e.V. \& DHWR e.V., 2020, S. 16).

${ }^{27}$ Die RVR bildet die Nachfolgeregelung für die 2008 aufgehobene gesetzliche Handelsklassensortierung für Rohholz (Forst-HKS), die im Jahr 1969 in Kraft gesetzt wurde.
} 
Stammholz bezeichnet den wirtschaftlich hochwertigsten Teil des Rohholzes, da es speziell für eine stoffliche Nutzung insbesondere in der Säge- oder Furnierindustrie vorgesehen ist. Es wird zum einen über die Länge weiter differenziert - dabei wird zwischen Stammholz-lang (zufällige Längen über $6 \mathrm{~m}=\mathrm{ST}$ ) und Stammholz-Abschnitten (einheitliche Bestelllängen von bis zu $6 \mathrm{~m}=\mathrm{FL}$ ) unterschieden. Zum anderen erfolgt eine Differenzierung über die Güte in Form einer Qualitätssortierung (Qualitätsklassen A bis D, siehe Tabelle 3). Ergänzend zu den bereits vorgestellten morphologischen Eigenschaften nehmen in der Sortierung von Stammholz weitere Faktoren Einfluss auf die Qualitätszuordnung. Darunter zählen neben Merkmalen der Stammform (Drehwuchs, Krümmung) und der Stirnfläche (z. B. Risse, Kern) auch Eigenschaften des Rindenbildes, wie beispielsweise die Anzahl und Form von überwallten Astnarben, Nekrosen oder auch Rindeneinschlüssen. Neben der Astigkeit (Erteld \& Achterberg, 1954, S. 577) wird auch die fakultative Verkernung (sog. Rotkern) als wesentlicher wertmindernder Faktor angesehen (Hapla \& Militz, 2008, S. 314).

Tabelle 3: Übersicht über die Qualitätssortierung von Buchenstammholz (eigene Darstellung in Anlehnung an die RVR, DFWR e.V. \& DHWR e.V., 2020, S. 25).

\begin{tabular}{|c|c|c|}
\hline $\begin{array}{c}\text { Qualitäts- } \\
\text { klasse }\end{array}$ & Allgemeine Beschreibung & $\begin{array}{c}\text { Buchenspezifische } \\
\text { Anforderungen (Auszüge) }\end{array}$ \\
\hline A & $\begin{array}{l}\text { Stammholz von ausgezeichneter Qualität. } \\
\text { Es ist fehlerfrei oder weist nur unbedeu- } \\
\text { tende Qualitätsmerkmale auf, die seine } \\
\text { Verwendung kaum beeinträchtigen. }\end{array}$ & $\begin{array}{l}\text { Drehwuchs max. } 2 \mathrm{~cm} / \mathrm{m} \text {; } \\
\text { einfache Krümmung max. } \\
2 \mathrm{~cm} / \mathrm{m} \text {; Risse unzulässig; } \\
\text { Rindenschäden unzulässig }\end{array}$ \\
\hline $\mathrm{B}$ & $\begin{array}{l}\text { Stammholz von normaler Qualität mit } \\
\text { wenigen und/oder mäßig ausgeprägten } \\
\text { Qualitätsmerkmalen. }\end{array}$ & $\begin{array}{l}\text { Sternriss max. } 2 / 3 \text { des } \\
\text { Durchmessers; gesunde } \\
\text { Äste } 2 \text { pro } 4 \mathrm{~m}\end{array}$ \\
\hline $\mathrm{C}$ & $\begin{array}{l}\text { Stammholz von normaler Qualität mit } \\
\text { vermehrt vorkommenden und/oder stär- } \\
\text { ker ausgeprägten Qualitätsmerkmalen. }\end{array}$ & $\begin{array}{l}\text { Äste in normalen Umfang } \\
\text { zulässig; Rotkern max. } \\
60 \% \text { des Durchmessers }\end{array}$ \\
\hline $\mathrm{D}$ & $\begin{array}{l}\text { Stammholz, das wegen seiner Merkmale } \\
\text { nicht den Klassen A, B oder C angehört, } \\
\text { aber als Stammholz nutzbar ist. }\end{array}$ & $\begin{array}{l}\text { Weißfäule max. } 50 \% \text { des } \\
\text { Durchmessers; keine weite- } \\
\text { ren Einschränkungen }\end{array}$ \\
\hline
\end{tabular}


Die mechanisierte Aufarbeitung von Buchenrohholz

Viele dieser Eigenschaften lassen sich schon am stehenden Stamm einschätzen und beurteilen. Eine Bewertungsmöglichkeit bietet die Einteilung von Willmann et al. (2001), die in der zweiten Bundeswaldinventur zum Einsatz kam. Sie ermöglicht die Klassifizierung des stehenden Buchenstammes in eine von sechs Güteklassen (absteigend von 1 bis 6; 1 = beste Qualitätsklasse).

Neben der Einteilung in ein Längen- und Qualitätssystem ist auch die Differenzierung über die Stärkeklasse bzw. Stammdimension von Bedeutung (Hapla \& Militz, 2008, S. 321). Je nach Stärkeklasse können unterschiedliche Produkte hergestellt werden, wie zum Beispiel Schälfurnier oder Möbelholz für Parkettböden oder den Modellbau. Aus diesem Grund liegen zwischen den unterschiedlichen Qualitätsklassen und in Abhängigkeit der Stärkeklasse erhebliche Preisunterschiede (Möhring, 1994, S. 116).

Das Sortiment Industrieholz umfasst den Teil des Rohholzes, welcher in der Regel mechanisch oder chemisch aufgeschlossen und für eine stoffliche Verwendung genutzt wird. Industrieholz wird daher vorrangig in der Holzwerkstoffindustrie bzw. in der Papier- und Zellstoffindustrie genutzt. Wie auch beim Stammholz erfolgt eine Längendifferenzierung in die Sorten Industrieholz-lang (über $3 \mathrm{~m}=\mathrm{IL}$ ) und Industrieholz-kurz (1 bis $3 \mathrm{~m}$ Länge = IS). Eine Ausnahme bilden Waldhackschnitzel, die bei einer stofflichen Verwertung ebenfalls zum Industrieholz gezählt werden. Auch beim Industrieholz erfolgt eine zusätzliche Einteilung in Qualitätsklassen. Im Gegensatz zum Stammholz ist die Sortierung aber nicht allzu komplex und es wird lediglich zwischen den Güteklassen N-normales Holz (gesund, nicht grobastig, keine starke Krümmung), F-fehlerhaftes Holz (leicht anbrüchig, grobastig, krumm) und K-krankes Holz (stark anbrüchig, jedoch gewerblich verwendbar) unterschieden. Die Qualitätsansprache erfolgt dabei nicht für jeden Abschnitt, sondern durchschnittsweise für einen Polter. Der Einfluss der morphologischen Eigenschaften der Buche fällt somit wesentlich geringer aus als bei der Qualitätsdifferenzierung des Buchenstammholzes.

Unter das Sortiment Energieholz fallen letztlich Sorten, welche für eine energetische Nutzung vorgesehen sind. Daneben zählt auch bei der Holzernte anfallendes Waldrestholz als auch Waldhackschnitzel (bei energetischer Nutzung) zum Energieholz. Die Längendifferenzierung wird analog zum Industrieholz durchgeführt (Energieholz-kurz $=$ BS, Energieholz-lang = BL). Eine zusätzliche Qualitätsdifferenzierung erfolgt nicht. 
Die mechanisierte Aufarbeitung von Buchenrohholz

\subsection{Arbeitsverfahren der Buchenholzernte}

Die vorangegangenen Kapitel widmeten sich der Beschreibung der morphologischen Eigenschaften der Buche und den daraus resultierenden Folgen für das Produkt Rohholz und dessen Sortierung. Darauf aufbauend soll sich nun mit historischen Aufarbeitungsverfahren der Buche beschäftigt und dabei die Vor- und Nachteile aufgezeigt werden, die aus einer mechanisierten Aufarbeitung resultieren.

Becker (1997, S. 76) definiert die Holzernte als „,technisches Instrument für die nachhaltige Bereitstellung des Rohstoffes Holz.“ Jede Holzernte bedeutet dabei stets einen aktiven Eingriff in die natürlichen Abläufe des Waldes, weshalb die genutzten Holzerntesysteme und Arbeitsverfahren gleichermaßen ökonomische, ökologische und soziale Kriterien erfüllen müssen (Bacher-Winterhalter, 2004, S. 5). Dabei werden neben den Ansprüchen eines naturnahen Waldbaus und einer nachhaltigen Bewirtschaftung auch die Wirtschaftlichkeit sowie Zertifizierungs-, Erholungs- und Naturschutzaspekte beachtet (Wehner \& Sauter, 2005, S. 301-302). Speziell die Anforderungen modernder Waldbaukonzepte wie der Dauerwaldbewirtschaftung beeinflussen dabei die Möglichkeiten und erfordern Anpassungen an die Holzernte (Bacher-Winterhalter, 2004, S. 6). Hinzu kommen Anforderungen, die aus den Eigenschaften des Bestandes resultieren, wie beispielsweise dem BHD des ausscheidenden Bestandes, der Topografie, dem Vorhandensein von Naturverjüngung oder auch der Verfügbarkeit von Unternehmen. Die Erfüllung dieser Ansprüche und der damit gekoppelten Anforderungen ist eng verbunden mit der Wahl des richtigen Arbeitsverfahrens ${ }^{28}$.

Historisch wurde die Buche bis in die sechziger Jahre des 20. Jahrhunderts rein manuell mittels Zugsäge aufgearbeitet (Hamberger, 2003, S. 33). Mit Einführung der Motorsäge änderte sich das Standardarbeitsverfahren hin zur motormanuellen Aufarbeitung. Mitte der achtziger Jahre des 20. Jahrhunderts wurde dann der Radharvester in Deutschland eingeführt, welcher sich spätestens durch die Aufarbeitung der Stürme „Vivian“ (1990) und „Wiebke“ (1990) in weiten Teilen Deutschlands etablieren konnte (Hamberger, 2003, S. 34; Höfle, 2002, S. 939). Ein bedeutender Grund für diese Veränderung ist der

\footnotetext{
${ }^{28}$ Ein Holzerntesystem setzt sich aus einem Aufarbeitungs- und Holzbringungsverfahren zusammen (Bacher-Winterhalter, 2004, S. 23).
} 
Die mechanisierte Aufarbeitung von Buchenrohholz

erhebliche Leistungsunterschied zwischen beiden Verfahren. Nach einer Studie von Ohrner (1999) besitzt ein Harvester bei einem mittleren Stückvolumen des ausscheidenden Bestandes von 0,2 Fm eine acht- bis zehnfache Leistung im Vergleich zur motormanuellen Aufarbeitung. Weithin bietet die mechanisierte Aufarbeitung eine wesentlich geringere Unfallgefahr für die ausführenden Arbeiter gegenüber der motormanuellen Holzernte: Wie Michels (2017, S. 211) in seiner Arbeit zeigen konnte, sinkt die Unfallgefahr beim Einsatz mechanisierter Aufarbeitungsverfahren im Vergleich zu motormanuellen erheblich. Da der Maschinenführer zudem den Fall des Baumes bei der Ernte besser kontrollieren kann, sinkt weiterhin die Gefahr, dass der Baum durch Fallschäden entwertet wird (Strandgard \& Mitchell, 2020, S. 1).

Bisher werden Harvester aufgrund der morphologischen Besonderheiten der Buche vor allem in schwächer dimensionierten Buchenbeständen eingesetzt (Guglhör, 1994, S. 698; Pausch, 2007, S. 746-748; Schorr, 2000, S. 522). Die technischen Entwicklungen der letzten Jahre erlauben aber zunehmend auch die Harvester-Nutzung in stärker dimensionierten Beständen (Böttcher et al., 2018, S. 27; Kleinschmit, 2015, S. 43). Kleinschmit (2015, S. 42) sieht dabei besonders die Weiterentwicklungen ,in den Bereichen des Aggregat-Handlings, der Durchzugsgeschwindigkeit sowie der Aggregat- und Kranleistung“" als entscheidend für die Vergrößerung des Einsatzspektrums an.

Die Auswahl eines Holzernteverfahrens erfordert nicht nur eine Prüfung und Abwägung der Anforderungen, sondern geht auch mit gewissen verfahrensabhängigen Problemen bzw. Grenzen einher. Dies betrifft insbesondere den eingeschränkten Aktionsradius eines Harvesters, welcher hauptsächlich von der Auslegerreichweite (Kranreichweite) und dem Rückegassenabstand des Bestandes bestimmt wird. Um die gesamte Hiebsfläche aufarbeiten zu können, wird bei einem Rückegassenabstand von 20 bis 25 m eine Kranreichweite von 15 m benötigt (Bacher-Winterhalter, 2004, S. 18). Entnahmebäume, die außerhalb dieser Reichweite stehen, müssen motormanuell zugefällt werden. Dies führt sowohl zu höheren Kosten (Bort et al., 1993, S. 124) als auch zu stärkeren Bestandesschäden (Morat et al., 1998, S. 102-104). Eine besondere Bedeutung kommt auch dem Thema Bodenpfleglichkeit bzw. Bodenverdichtung zu (Heindl \& Pausch, 2007, S. 6; Labelle \& Jaeger, 2011, S. 2314): Durch den Einsatz schwerer Forstmaschinen kommt es demnach $\mathrm{zu}$ einer Verdichtung des Bodens und einer damit verbundenen Beeinträchtigung der Wurzelsysteme. 


\subsection{Produktivitätsmodelle der mechanisierten Holzaufarbeitung}

Die vorliegende Arbeit behandelt die Erstellung eines Produktivitätsmodelles der mechanisierten Holzaufarbeitung von Buchen via Harvester. Ein Harvestersystem beschreibt genau einen Maschinenführer auf genau einer Maschine. Wenn im Mehrschichtbetrieb gearbeitet wird, handelt es sich um zwei oder mehr Harvestersysteme, die jedoch dasselbe Arbeitsmittel als Gemeinsamkeit besitzen. Auf diese Harvestersysteme wirken eine Reihe von Einflussfaktoren. Dazu zählen neben den Bestandesmerkmalen auch die Aushaltungsanweisungen sowie die Eigenschaften des Harvester und die Eignung des Maschinenführers (u. a. Nurminen et al., 2006, S. 336-337; Ovaskainen, 2009, S. 8-15; Visser et al., 2009, S. 1-3). Grundlegend können in der Erstellung eines Produktivitätsmodells für Harvestersysteme zwei Ansätze verfolgt werden, die sich in der Basis ihrer Datengrundlage unterscheiden (Cremer, 2008, S. 9-17). Auf diese soll nachfolgend ein Blick geworfen werden.

\subsubsection{Modelle auf der Basis von Bestandesdaten}

Der erste Ansatz verfolgt die Nutzung von bestehenden Daten in einem meta-analytischen Konzept. Dabei wird vor allem auf Leistungsnachweise der Maschinenbuchführung oder Tagesaufschriebe der Maschinenführer zurückgegriffen. Grundlegende Arbeiten in diesem Bereich wurden von Ohrner (1999) und Heinimann (2001) durchgeführt, die für ihre Modelle jeweils rund 2.000 Leistungsnachweise für den Einsatz verschiedener Harvestersysteme beim Einschlag von Rundholz analysiert haben. ${ }^{29}$ Klassischerweise spiegelt jeder Leistungsnachweis dabei einen Bestand mit seinen jeweiligen Eigenschaften - wie zum Beispiel dem mittleren Baumvolumen des ausscheidenden Bestandes - wider (Heinimann, 2001, S. 2). Die Modelle verstehen sich dadurch als Bestandesmodelle und können daher keine Aussagen über den Einfluss von Einzelbaumparametern auf die Produktivität treffen. ${ }^{30}$ Beide Studien kommen zu dem Schluss, dass eine hohe Abhängigkeit

\footnotetext{
${ }^{29}$ Die untersuchten Leistungsnachweise wurden vorrangig in Fichten und Kiefernbeständen erzeugt.

${ }^{30}$ Eine Ausnahme besteht, wenn die mittlere Ausprägung eines Baumparameters für den Bestand geschätzt oder gemessen und in das Modell mitaufgenommen wird.
} 
Die mechanisierte Aufarbeitung von Buchenrohholz

zwischen der Produktivität und dem Baumvolumen besteht, wobei die Stärke dieser Abhängigkeit mit steigendem Baumvolumen abnimmt. Als weitere Erkenntnis konnte festgestellt werden, dass die Produktivität mit steigendem Entwicklungsgrad der eingesetzten Harvester ansteigt (Heinimann, 2001, S. 6).

Als weitere Untersuchung mit einem meta-analytischen Ansatz kann die Studie von Pausch und Ponitz (2002) angeführt werden. Die Grundlage dieser Untersuchung bildeten rund 450 Tagesaufschriebe von 12 unterschiedlichen Harvestersystemen, welche sich zusätzlich zwischen dem Einsatz in Nadel- und Laubholzbestände differenzieren lassen. Eine weitere Besonderheit dieser Studie ist die Vielzahl an zusätzlichen Variablen, die in die Regressionsanalyse mit einbezogen wurden. Dabei konnten die Autoren nicht nur den Einfluss des mittleren Baumvolumens auf die Produktivität bestätigen - zusätzlich dazu fanden sie auch einen Einfluss der mittleren Fahrstrecke pro Baum, der Baumart, des Anteils der grünen Krone an der Baumhöhe, des X-Holz-Anteils sowie der mittleren Anzahl an Sortenstücken pro Baum. ${ }^{31}$

Eine neuere und umfangreiche Untersuchung in diesem Gebiet wurde von Eriksson und Lindroos (2014) durchgeführt. Über eine dreijährige Periode wurden die Daten von über 700 Harvestersysteme ${ }^{32}$ gesammelt und analysiert. Dabei konnten entscheidende Kennzahlen für die Produktivitätsdifferenzen zwischen Durchforstungs- und Erntebeständen abgeleitet werden.

\subsubsection{Modelle auf Einzelbaumbasis}

Der zweite Ansatz zur Erstellung von Produktivitätsmodellen für Harvestersysteme beruht auf der Nutzung von Einzelbaumdaten (sog. Einzelbaummodelle). In der Vergangenheit wurden bereits zahlreiche - in vielen Fällen maschinenspezifische - Produktivitätsmodelle auf Einzelbaumbasis erstellt (u. a. Cremer, 2008, S. 14-17; Purfürst, 2009, S. 24-26). Um einen besseren Überblick über den bestehenden Forschungsstand und die wissenschaftliche Lücke dieser Arbeit zu bieten, sollen in Anlehnung an Purfürst (2009, S. 27-31) und anhand der in Kapitel 3.3 angesprochen leistungsbeeinflussenden Faktoren

\footnotetext{
${ }^{31}$ Die Ausprägungen der Einflussgrößen stellen mittlere Werte dar, die während eines Tages- oder einer Arbeitsschicht aufgetreten sind. Das mittlere Baumvolumen beinhaltet bei dieser Studie auch das X-Holz (Pausch, 2002, S. 100-101).

${ }^{32}$ Mehr als 20.000 Hiebe mit einem Gesamteinschlagsvolumen von über 20 Mio. Fm.
} 
die bestehenden Studien kurz vorgestellt werden. Die vorgestellten Befunde beruhen dabei nicht ausschließlich auf Einzelbaummodellen, sondern schließen einzelne wichtige Ergebnisse aus Bestandesmodellen mit ein, um einen umfassenden Blick auf die Stärke und Auswirkung von leistungsbeeinflussenden Faktoren zu gewährleisten.

\subsubsection{Bedeutung von leistungsbeeinflussenden Faktoren}

\section{Input}

Die Input-Variablen beschreiben die Baumeigenschaften. Hierzu zählen neben der Baumart und dem Volumen der Entnahmebäume auch deren Qualität, der Kronentyp sowie weitere Kronenparameter wie die Aststärke, der Astablaufwinkel, die Zwieseligkeit oder ein möglicher Splitterbefall (Purfürst, 2009, S. 28).

Da sich die meisten Untersuchungen auf Nadelholz (vorrangig Kiefer, Pinus spec. L. und Fichte, Picea spec. L.; u. a. Alam et al., 2014; Heinimann, 2001; Kärhä et al., 2004; Nurminen et al., 2006, 2009; Ovaskainen et al., 2004; Pöhler, 2003; Spinelli et al., 2009; Suadicani \& Nordfjell, 2003) beschränken, gibt es nur wenige Modelle, die Laubbaumarten und speziell die Buche einbeziehen. Grund dafür ist zum einen der bisherige Einsatzschwerpunkt des Harvesters in nadelholzdominierten Wäldern (vgl. Kapitel 4.3) und zum anderen die negativen morphologischen Eigenschaften von Laubhölzern, welche die mechanisierte Aufarbeitung zusätzlich erschweren (Liepiņš et al., 2015, S. 494).

Die wenigen existierenden Laubholzstudien beschäftigen sich weiterhin oft mit besonderen Baumarten oder Bewirtschaftungsformen. So führten beispielsweise Suchomel et al. (2012) einen Vergleich zwischen Harvester- und Baggeraufarbeitung in italienischen Niederwäldern durch, die vorrangig aus Kastanie (Castanea sativa Mill. L.) bestanden. ${ }^{33}$ Einen ähnlichen Vergleich führten Suchomel et al. bereits in Eichen-dominierten Niederwäldern durch (Suchomel, 2010; Suchomel et al., 2011). Eine Studie von Liepiņš et al. (2015) beschäftigte sich mit der mechanisierten Aufarbeitung von Grau-Erle (Alnus incana L.) in Lettland. Hinzu kommen einzelne Veröffentlichungen über die mechanisierte Aufarbeitung von Pappel, hauptsächlich verortet im italienischen Raum (Danilović et al., 2011; Spinelli, Magagnotti \& Nati, 2011; Spinelli, Magagnotti, Sperandio et al., 2011)

\footnotetext{
${ }^{33}$ Die Bäume in dieser Studie wurden alle motormanuell gefällt und im Anschluss vorgerückt. Im Fokus der Untersuchung stand nur die Aufarbeitung, die außerhalb der Versuchsflächen erfolgte (Suchomel et al., 2012, S. 39-40).
} 
Die mechanisierte Aufarbeitung von Buchenrohholz

oder die mechanisierte Aufarbeitung von Birkenüberhältern (Grönlund \& Eliasson, 2019).

Aus den bestehenden Studien lässt sich ein signifikanter Einfluss der Baumart erkennen.

Dieser wird auch in gemischten Einzelbaummodellen bestätigt, in denen die Baumart als unabhängige Variable mit in die Analyse einbezogen wurde. So besaß die Baumart beispielsweise im Modell von Cremer (2008, S. 64) einen signifikanten Einfluss auf die Produktivität. ${ }^{34}$ Demnach ist bei der Aufarbeitung von Laubbäumen ab einem BHD über 30 $\mathrm{cm}$ mit einem Produktivitätsverlust im Vergleich $\mathrm{zu}$ Nadelholz $\mathrm{zu}$ rechnen (Cremer, 2008, S. 75). Eine Untersuchung von Nurminen et al. (2006, S. 345) kam zu ähnlichen Befunden. ${ }^{35}$ Produktivitätsmodelle für die mechanisierte Aufarbeitung von Buche wurden bisher in nur wenigen Versuchen thematisiert (u. a. Böttcher et al., 2018; Guglhör, 1994; Kleinschmit, 2015; Pausch, 2002; Schorr, 2000).

Da jede Studie nur einen gewissen volumenbezogenen Geltungsbereich hat, muss der Einfluss des mittleren Baumvolumens des ausscheidenden Bestandes zwingend berücksichtigt werden, um die Baumartenunterschiede (speziell der Buche) besser quantifizieren zu können. Der überproportional starke Effekt dieses Faktors auf die Zeit der mechanisierten Aufarbeitung ist allgemein bestätigt (Alam et al., 2014; Nakagawa et al., 2007; Nurminen et al., 2006; Ovaskainen et al., 2004). Weiterhin konnte belegt werden, dass es sich hierbei um einen nicht linearen Zusammenhang handelt sowie dass ein maschinenabhängiges optimales Baumvolumen existiert, in dem die Produktivität am höchsten ist. Dieser Zusammenhang konnte sowohl für Nadelholz (Kärhä et al., 2004; Labelle \& Huss, 2018; Nurminen et al., 2006) als auch für Laubholz (Guglhör, 1994; Pausch, 2002; Schorr, 2000) bestätigt werden. Übersteigt das Baumvolumen diesen optimalen Punkt, sinkt der Produktivitätsverlauf im Zuge einer negativen Grenzleistung ab. An diesem Punkt lässt sich der Kenntnisstand der mechanisierten Aufarbeitung mit dem klassischen Stückvolumengesetz verbinden (Kapitel 3.3). Sollte diese Theorie der negativen Grenzproduktivität zutreffen, dann muss die Annahme eines proportionalen Zusammenhangs zwischen der Stückzeit und dem Stückvolumen durch einen progressiven Zusammenhang

\footnotetext{
${ }^{34}$ In den Vergleich wurde die Aufarbeitungszeit von 93 Laubholz-, 857 Fichten- und 324 Kiefernbeständen einbezogen. Eine genauere Differenzierung der Laubholzbestände erfolgte nicht (Cremer, 2008, S. 62-64).

${ }^{35}$ Der Vergleich erfolgte hier auf Einzelbaumebene. Das Modell beruht auf den Aufarbeitungszeiten von 276 Fichten, 272 Kiefern und 88 Birken (Nurminen et al., 2006, S. 341).
} 
ersetzt werden. Fraglich ist aber, ob sich durch eine Veränderung der Baumart die Funktionsverläufe der Harvesterproduktivität verschiedener Baumarten in Gänze oder nur in gewissen Volumenbereichen voneinander unterscheiden.

In den wenigen vorhandenen Buchenstudien kann diesbezüglich ein Konsens festgestellt werden: Bis zu einem mittleren Baumvolumen des ausscheidenden Bestandes von 0,4 Fm liegt die Leistung der hochmechanisierten Erntemaschinen in Laubholzbeständen auf dem gleichen Niveau wie in Nadelholzbeständen (Pausch, 2002, S. 122; Pausch \& Ponitz, 2002, S. 12). Guglhör (1994, S. 698) verglich in seiner Studie die mechanisierte Aufarbeitung von Buchen, Fichten und Lärchen und stellte fest, dass bis zu einem mittleren Baumvolumen von 0,5 Fm wipfelschäftige Buchen eine vergleichbare Zeit benötigen wie Nadelholzarten. Ab einem höheren Volumen mindern Starkäste, Zwiesel und krumme Schäfte die Aufarbeitungsleistung der hochmechanisierten Ernte in Laubholzbeständen (Schorr, 2000, S. 522). Guglhör (1995) und Pausch (1999) sehen besonders die Aufarbeitung von Buchen mit einem Baumvolumen von über 1,5 Fm als sehr kritisch an. Studien, die sich auf diesen Volumenbereich konzentrieren, sind allerdings nicht vorhanden. Es wird dennoch sichtbar, dass sich die Produktivität der mechanisierten Buchenaufarbeitung bis zu einem gewissen Punkt auf dem Niveau von Nadelholzbeständen bewegt, bis die morphologischen Besonderheiten der Baumart einen Mehraufwand verursachen.

Es liegt somit die Frage nahe, welche morphologischen Qualitätsmerkmale den zusätzlichen Mehraufwand einer Baumart verursachen und wie stark dieser ausfällt (vgl. Labelle et al., 2016, S. 175). Eine bereits vorgestellte Möglichkeit zur Analyse dieses Effekts bietet die Kategorisierung des Kronentyps (Kapitel 4.1). Kleinschmit (2015, S. 51) konnte in seiner Dissertation nachweisen, dass der Kronentyp einen signifikanten Einfluss auf die mechanisierte Aufarbeitungszeit besitzt. ${ }^{36}$ Er konnte weiterhin belegen, dass sich die Aufarbeitungszeit um 20 \% erhöht, wenn der Baum statt eines wipfelförmigen einen besenförmigen Kronentyp aufweist. Ist der Baum zwieselig, steigt die Aufarbeitungszeit im Durchschnitt um weitere $13 \%$ an (Kleinschmit, 2015, S. 52). Diese Ergebnisse bestätigen einen frühen Versuch von Guglhör (1994, S. 698), in welchem er den Kronentyp in seine Analysen integrierte. Auch Schorr (2000, S. 522) schloss die Kronenform in sein

\footnotetext{
${ }^{36}$ Die untersuchten Bäume befanden sich in einem BHD-Bereich von 20 bis $50 \mathrm{~cm}$. In die Analyse wurde die Aufarbeitungszeit von 62 wipfelschäftigen, 168 zwieseligen und 177 besenartigen Buchen berücksichtigt (Kleinschmit, 2015, S. 51).
} 
Die mechanisierte Aufarbeitung von Buchenrohholz

Analysemodell mit ein, unterschied dabei aber zwischen fein-, grobastigen und zwieseligen Buchen.

Anzumerken ist weiterhin, dass die reine Bewertung des Kronentyps viele morphologische Faktoren außer Acht lässt (vgl. Kapitel 4.2), welche die mechanisierte Aufarbeitung ebenfalls beeinflussen können - so zum Beispiel die Geradschaftigkeit und Astigkeit. Aus diesem Grund untersuchten Labelle et al. (2016) die mechanisierte Aufarbeitung von Harthölzern (vorrangig Acer saccharum L.) im Hinblick auf die Auswirkungen unterschiedlicher Qualitätsparameter. Die Bäume wurden dazu in Abhängigkeit ihrer allgemeinen Stammform, ihres Kronentyps, ihrer Geradschaftigkeit und ihrer potentiellen Sortenausbeute eingeschätzt und in eine von acht Qualitätsklassen eingeteilt (Labelle et al., 2016, S. 178). Es zeigte sich hierbei, dass die Produktivität bei Bäumen mit schlechter Qualität um 15-20 \% im Vergleich zu Bäumen mit guter Qualität sank.

Zuletzt sollen an dieser Stelle zwei Untersuchungen vorgestellt werden, die weitere Qualitätsmerkmale des Baumes in ihren Produktivitätsmodellen berücksichtigten. Suchomel et al. (2012, S. 41) integrierten etwa eine Kovariable, die den Effekt der allgemeinen Baumform als eine fünfstufige Variable abbildet. Die Einteilung orientiert sich an einer Studie von Spinelli et al. (2002) und schätzt den Baum zwischen Stufe 1 (kleine Äste, gerader Stamm) bis hin zu Stufe 5 (stark gezwieselt und oder viele starke Hauptäste) ein. Eine weitere Möglichkeit ist die Bewertung der Bäume nach ihrer Kraftschen Klasse (Kraft, 1884), um den sozialen Status des Baumes abzuschätzen. Dieses Klassensystem wurde in einer Studie von Suchomel et al. (2011, S. 292-293) in das Produktivitätsmodell integriert und zeigte einen signifikanten Einfluss auf die Produktivität.

\section{Umweltbedingungen}

Unter die Kategorie Umweltbedingungen fallen sowohl die Flächenverhältnisse als auch klimatische Bedingungen während des Arbeitseinsatzes (Purfürst, 2009, S. 28-29). Eine Einflussgröße, die dabei stark von den Input-Variablen Baumart und Baumvolumen abhängt, ist die Bestandesdichte. Steigt diese an, können beispielsweise mehrere Entnahmebäume von einer Harvesterposition aus erreicht werden, was in einer geringeren Fahrentfernung resultiert (Glöde \& Sikström, 2001, S. 80).

Der Fahrtweg zwischen den Entnahmebäumen wird zusätzlich durch den Auszeichnungstyp und das Entnahmeprozent beeinflusst. Studien wie von Stampfer und Steinmüller 
(2004) konnten zeigen, dass die Produktivität mit steigender Fahrentfernung abnimmt. ${ }^{37}$ Die Geländeneigung kann sowohl einen Einfluss auf die Wahl des Arbeitsverfahrens als auch auf die Produktivität besitzen (Stampfer \& Steinmüller, 2004, S. 9). Die in der Studie von Pausch und Ponitz (2002) untersuchten Radharvester ${ }^{38}$ zeigten jedoch bis zu einer Geländeneigung von 25 - $30 \%$ keine Produktivitätseinbußen.

Weitere einflussnehmende Umweltbedingungen sind nach Purfürst (2009, S. 29): „Sichtverhältnisse, Bestockung, Unterwuchs, Schichtigkeit, Tragfähigkeit des Bodens, Relief, Tageszeit, Jahreszeit und Wetter.“

\section{Arbeitsaufgabe}

Unter diesen Aspekt fallen Restriktionen bzw. Anweisungen, welche die Arbeitsweise des Arbeitssystems beeinflussen. Diese sind in der Praxis sehr individuell und umfangreich und lassen sich nur schwer definieren bzw. voneinander abgrenzen. Besonders treten dabei jedoch der Zustand bzw. Umfang der Bestandesvorbereitung sowie die Eingriffsstärke hervor (Purfürst, 2009, S. 29-30).

Nach einer Studie von Borr und Pfeuffer (1999, S. 90) lässt sich beispielsweise die Produktivität um rund $16 \%$ steigern, wenn Z-Bäume und ausscheidender Bestand gut sichtbar ausgezeichnet wurden. Ein positiver Effekt auf die Produktivität geht weiterhin mit einer Erhöhung der Eingriffsstärke einher. Dabei verringert sich zum einen die mittlere Fahrentfernung zwischen den Entnahmebäumen und zum anderen kann der Fahrer durch die daraus resultierende geringere Bestandesdichte besser innerhalb der Fläche agieren (Stampfer, 2001).

Zusätzlich ist auch die Wahl des richtigen Arbeitsverfahrens bzw. das Zusammenspiel zwischen Harvester und der anschließenden Rückung von Bedeutung. In vielen Schlägen passt der Maschinenführer seine Arbeitsweise so an, dass er die aufgearbeiteten Abschnitte bereits vorsortiert und zu Raupoltern zusammenlegt (Vorrücken), um die Rückung zu vereinfachen. In einer Studie von Väätäinen et al. (2006) konnte gezeigt werden, dass eine solche Arbeitsweise die Produktivität des Harvesters zwar reduziert, die Produktivität des Gesamtprozesses hingegen steigert.

\footnotetext{
${ }^{37}$ Das Arbeitsmittel dieser Studie waren Kettenharvester der Firma Valmet (Produktbezeichnung: Valmet 911.1 X3M).

${ }^{38}$ Radharvester FMG 707/280 ÖSA, ausgestattet mit Bogiebändern (Pausch, 2002, S. 93).
} 
Die mechanisierte Aufarbeitung von Buchenrohholz

\section{Arbeitssystem}

Die Einflussgröße Arbeitssystem teilt sich in die leistungsbeeinflussenden Eigenschaften des Harvesters (Arbeitsmittel), des Maschinenführers sowie deren Zusammenspiel auf. Die Harvestereigenschaften umfassen die technischen Merkmale des Untersuchungsobjektes. Dazu gehören beispielsweise die Motorisierung, die hydraulische Kraft, das Harvesteraggregat (maximaler Öffnungsdurchmesser, Vorschubkraft und -geschwindigkeit, Entastungsmesser und Sägeausstattung) sowie die Kranausstattung (Reichweite, Hubkraft, Nurminen et al., 2006, S. 337). Der genaue Einfluss der technischen Eigenschaften wurde bisher nur in wenigen Studien untersucht. Diese legen jedoch nahe, dass die Leistungsstärke der Maschine (bzw. ihre Motorleistung) ${ }^{39}$ als wichtigste Einflussgröße verstanden werden kann (Jiroušek et al., 2007, S. 480-481; Spinelli et al., 2010, S. 232-235). Eine Studie von Lindroos et al. (2008) stellte wiederum die Kranausstattung in den Fokus und konnte belegen, dass die Reichweite und die Hubkraft des Kranarmes ebenfalls bedeutsame Einflussgrößen sind. Auch spezielle Eigenschaften des Harvesteraggregates, wie etwa die Vorschubgeschwindigkeit (Nuutinen et al., 2010) oder die Wartungsintensität (Gerasimov et al., 2012), haben einen signifikanten Effekt auf die Produktivität. Unter den leistungsbeeinflussenden Eigenschaften des Maschinenführers versteht man wiederum:

(1) die mentale Leistungsfähigkeit (Gellerstedt, 1997; Nåbo, 1990, in Alam et al. 2014, S. 14),

(2) die physische Leistungsfähigkeit (Nicholls et al., 2004) und

(3) die Arbeitstechnik (Aufarbeitungsabläufe, „Pfad“ durch die Fläche, Ovaskainen et al., 2004; Ovaskainen, 2009).

Viele Studien gehen davon aus, dass die Operator performance neben dem Baumvolumen der zweitstärkste Faktor in Bezug auf die Leistung eines Harvestersystems ist (u. a. Malinen et al., 2018; Ottaviani Aalmo et al., 2020; Purfürst, 2009; Purfürst \& Erler, 2011). So wiesen Kärhä et al. (2004) nach, dass die Produktivität um bis zu 40 \% variiert, wenn verschiedene Maschinenführer mit demselben Harvester bei gleichen Bestandesbedingungen arbeiten. In einer Studie von Ovaskainen et al. (2004) schwankte die Leistung sogar um bis zu $55 \%$ zwischen den verschiedenen Maschinenführern. Zusätzlich konnte

\footnotetext{
${ }^{39}$ In Deutschland wird diesbezüglich auch der Begriff der Leistungsklasse verwendet. Nach dem KWF gehören Radharvester mit einer Motorleistung von bis zu 140 kW zur Leistungsklasse 1, über 140 bis $180 \mathrm{~kW}$ zur Klasse 2 und über $180 \mathrm{~kW}$ zur Klasse 3 (Forbrig, 2000, S. 98).
} 
Purfürst (2009) in seiner Dissertation aufzeigen, dass sich die Leistung eines Maschinenführers in der Zeit seiner Ausbildung mitunter verdoppeln kann. Welche Eigenschaften einen ,guten“ Fahrer ausmachen, sind nicht genau geklärt. Es wird jedoch vermutet, dass sich dieser insbesondere durch die Überlappung mehrere Tätigkeiten (Harstela, 2004), eine hohe Kranaktivität (Väätäinen et al., 2004) sowie eine vorausschauende Planung (Ranta, 2004) charakterisieren lässt.

\section{Output}

Unter die Output-Variablen fällt die Anzahl der ausgehaltenen Sortimente pro Baum (Einzelbaummodell) bzw. die Sortimentsvielfallt eines Schlages (Bestandesmodell). Diese sind stark abhängig von der Aushaltungsanweisung, welche Informationen über die Auswahl der Sorten sowie deren spezifische Längen-, Durchmesser- und Qualitätsbedingungen enthält. Zusätzlich fällt darunter auch die Priorisierung zwischen den unterschiedlichen Sorten.

Die Anzahl unterschiedlicher Sortimente kann beispielsweise die Schnittzahl pro Baum bzw. die Anzahl an Abschnitten pro Baum erhöhen sowie beeinflussen, ob der Harvester die Sortimente für die Rückung vorsortiert. ${ }^{40}$ Der Einfluss konnte sowohl in Studien auf Bestandesebene (Pausch \& Ponitz, 2002; Sirén \& Aaltio, 2003) als auch in Studien auf Einzelbaumbasis bestätigt werden (Alam et al., 2014; Nurminen et al., 2006). Diese beziehen sich jedoch weitestgehend auf nadelholzdominierte Wälder.

\section{Weiterentwickelte Anwendungsoptionen}

Ein erstelltes Produktivitätsmodell kann auch genutzt werden, um weitere Anwendungsoptionen zu ermöglichen. Zum einen können Kalkulationsprogramme erstellt werden, die es ermöglichen, verschiedene Modelle zusammenzufassen und dem Anwender in einer benutzerfreundlichen Form zur Verfügung zu stellen (Purfürst, 2009, S. 32). So entwickelten beispielsweise Erni et al. (2004) bzw. Erni und Frutig (2004) ein Excelbasiertes Kalkulationsprogramm („HeProMo“) für die Aufarbeitung mittels Radharvester für den Schweizer Raum, welches mittlerweile vielfach weiterentwickelt wurde (Fischer \& Stadelmann, 2019).

\footnotetext{
${ }^{40}$ Diese Tätigkeit wird im deutschsprachigen Raum auch Vorrücken genannt. In englischsprachigen Studien wird eher die Begrifflichkeit Product handling/management genutzt (Suchomel et al., 2011, S. 292; Suchomel et al., 2012, S. 41).
} 
Die mechanisierte Aufarbeitung von Buchenrohholz

Zum anderen können Produktivitätsmodelle im Rahmen einer Simulationssoftware genutzt werden. Diese reichen von einfachen analytischen Simulatoren (Asikainen, 1995; Eliasson, 1999; Schöpfer et al., 2004; Wang \& Greene, 1999; Wang \& LeDoux, 2003) bis hin zu sehr komplexen graphischen Programmen, in denen der Einsatz von verschiedenen Verfahren in Beständen simuliert werden kann (Hemm et al., 2006; Ziesak et al., 2004).

\subsection{Kapitelzusammenfassung}

Dieses Kapitel beschäftigte sich mit der technischen Produktion von Buchenrohholz. In der Verbindung der ersten beiden Unterkapitel konnte gezeigt werden, dass die besondere Wuchsdynamik und die artspezifische Kronenausbildung der Buche die Möglichkeiten der Aushaltung sowie die Eigenschaften des Rohholzes bestimmen. Diese morphologischen Besonderheiten grenzen die Buche von anderen Baumarten - speziell jedoch von Nadelgehölzen - ab, da sie zusätzlich auch die Auswahl der Aufarbeitungsverfahren für das Rohholz beeinflussen (Kapitel 4.3). Den Abschluss des Kapitels bildete eine Zusammenfassung der bestehenden Studien, die sich mit der Erstellung von Produktivitätsmodellen bei der mechanisierten Holzaufarbeitung auseinandergesetzt haben. Die Übersicht der Studien erfolgte in Anlehnung an die leistungsbeeinflussenden Faktoren, die bereits in Kapitel 3.3.3 vorgestellt wurden. Dabei konnte eine Forschungslücke in Bezug auf Arbeiten zum Einsatz von Harvestern in stärker dimensionierten Buchenbeständen aufgezeigt werden.

Mit diesem Kapitel schließt der Theorieteil dieser Arbeit ab. Im nachfolgenden Kapitel wird das Forschungsinteresse dieser Arbeit und die Ableitung der daraus resultierenden Forschungshypothesen zusammenfassend beschrieben. 


\section{Forschungshypothesen}

Im Fokus dieser Arbeit steht die Ermittlung der prozessabhängigen Kosten der mechanisierten Buchenaufarbeitung. Hierzu wird der Prozess zunächst zerlegt und in einzelnen Aktivitäten separat analysiert, um auf diese Weise die wichtigsten Kostentreiber zu identifizieren. Anhand einer simulierten Feldstudie werden die Auswirkungen dieser zunächst isoliert betrachteten einzelnen Aktivitäten exemplarisch auf Bestandesebene quantifiziert. Damit wird eine relevante wissenschaftliche (Kapitel 4.4.3) und forstbetriebliche (Kapitel 2.3) Forschungslücke geschlossen. Das Vorgehen orientiert sich dabei an den folgenden sechs Forschungshypothesen, die im Nachgang in ihrem theoretischen und empirischen Kontext eingebettet werden:

Vorangegangene Studien konnten nachweisen, dass sich der Prozess der mechanisierten Aufarbeitung in seine Aktivitäten zerlegen lässt und jede Aktivität einzeln über ein forstliches Produktivitätsmodell beschrieben werden kann (Alam et al., 2014, S. 19; Nurminen et al., 2006, S. 347-348, 2009, S. 854). Die bisherigen Studien, beschränken sich aber auf nadelholzdominierte Wälder. Mit diesem Hintergrundwissen wird folgende Hypothese abgeleitet:

H1: Der Prozess der mechanisierten Buchenaufarbeitung lässt sich in verschiedene Aktivitäten zerlegen, wobei jede dieser Aktivitäten mit einem separaten Produktivitätsmodell beschrieben werden kann.

Wie der bisherige Forschungsstand weiterhin zeigt, kommt es bei der mechanisierten Aufarbeitung zu einem maschinen- und baumartenabhängigen Punkt der maximalen Produktivität bzw. existiert ein Baumvolumen mit optimaler Produktivität (Leistungsgrenze, Purfürst, 2009, S. 88). Übersteigt das Baumvolumen diese Grenze, sinkt die Produktivität wieder ab. Laut den bestehenden Studien liegt dieser Punkt für die mechanisierte Aufarbeitung von Buche bei einem Baumvolumen von rund 0,5 Efm (Guglhör, 1994, S. 697; Schorr, 2000, S. 522). Beide Studien haben aber nur wenige Einzelbäume oberhalb dieser Grenze erfasst. Die zweite Hypothese bezieht sich daher auf die Analyse der Grenzproduktivität und lautet dabei wie folgt: 
Forschungshypothesen

H2: Die Produktivität der mechanisierten Buchenaufarbeitung steigt mit zunehmender Baumdimension bis zum Punkt der Leistungsgrenze an und sinkt im Zuge der negativen Grenzproduktivität danach wieder ab.

Als wichtige Ursache der negativen Grenzproduktivität bei der mechanisierten Aufarbeitung von Buche wird der Einfluss der Kronenform und der Stammqualität angegeben (Kleinschmit, 2015, S. 52; Pausch, 2002, S. 86; Schorr, 2000, S. 522). Diese Faktoren können sowohl die allgemeine Aufarbeitungsgeschwindigkeit verringern als auch ein Umgreifen des Aggregates erzwingen. In bisherigen Untersuchungen erfolgte die Einschätzung der beiden Parameter baumartenübergreifend sehr differenziert (u. a. Labelle et al., 2016, S. 175; Suchomel et al., 2012, S. 41). Wie Kapitel 4.1 zeigte, empfiehlt sich für die Buche eine Einteilung in Form von drei Kronentypen. Darüber hinaus ist eine Einschätzung der äußeren Stammqualität wichtig, da diese nicht nur Einfluss auf die Produktivität, sondern auch auf die Aushaltungsmöglichkeiten nimmt (Kapitel 4.2). Aus diesen Überlegungen leitet sich die dritte Forschungshypothese ab:

H3: Neben dem Baumvolumen bilden die Kronenform und die Qualität des Stammes wichtige Kostentreiber bei der mechanisierten Buchenaufarbeitung.

Weiterhin existieren zahlreiche Aushaltungsmöglichkeiten bei der Aufarbeitung eines Buchenstammes (Kapitel 4.2). Dabei konnten bestehende Forschungsarbeiten einen signifikanten Einfluss der Aushaltung auf die Produktivität belegen (u. a. Alam et al., 2014, S. 20; Eriksson \& Lindroos, 2014, S. 185; Sirén \& Aaltio, 2003, S. 42). Nurminen et al. (2006, S. 344) geben beispielsweise an, dass jedes zusätzlich auszuhaltende Sortiment die Entastungszeit eines Baumes um rund 10 \% erhöht. Dieser Effekt wurde allerdings bisher nur für nadelholzdominierte Wälder bestätigt. Aus diesem Grund lautet die vierte Forschungshypothese:

H4: Eine steigende Komplexität der Aushaltung (Art und Anzahl der Sortimente) vermindert die Produktivität der mechanisierten Buchenaufarbeitung.

Der bestehende Forschungsstand lässt zudem darauf schließen, dass sich die Produktivität der mechanisierten Buchenaufarbeitung bei geringeren Baumvolumina auf dem gleichen Niveau bewegt wie bei der Aufarbeitung von Nadelholz (Cremer, 2008, S. 75; Guglhör, 
1994, S. 698; Pausch, 2002, S. 122). Danach fällt die Produktivität im Vergleich zum Nadelholz ab. Gulghör (1994, S. 698) beschreibt, dass dieser Punkt für wipfelschäftige Buchen bei einem Baumvolumen von 0,5 Fm zu erwarten ist - Pausch (2002, S. 122) sieht eine beginnende Differenz hingegen bereits ab 0,4 Fm. Die fünfte Forschungshypothese zielt aus diesem Grund auf den Vergleich mit bestehenden Produktivitätsmodellen ab und postuliert:

H5a: Das Produktivitätsmodell dieser Arbeit lässt sich in die bestehenden Modelle der mechanisierten Buchen- bzw. Laubholzaufarbeitung einordnen.

H5b: Die Produktivität der mechanisierten Buchenaufarbeitung ist geringer als in vergleichbaren Studien aus nadelholzdominierten Wäldern.

Das Fundament einer Zeitstudie ist die Definition und Abgrenzung der im Prozess integrierten Aktivitäten (Kapitel 3.4). Der Detailgrad der Untersuchung spielt dabei eine entscheidende Rolle: Die meisten Studien, die sich mit der mechanisierten Aufarbeitung auseinandersetzen, betrachten den Prozess auf einer Makroebene und unterscheiden nur zwischen wenigen Aktivitäten (z. B. Anfahrt, Fällung und Aufarbeitung; u. a. von Bodelschwingh, 2006, S. 100; Purfürst, 2009, S. 53). Dies hat zur Folge, dass genaue Aussagen über die Stärke von leistungsbeeinflussenden Faktoren auf einzelne Aktivitäten des Aufarbeitungsprozesses limitiert sind und der genaue Zeitpunkt der Einflussnahme nicht bestimmt werden kann. Ein Grund dafür, dass nur wenige Studien den Prozess auf einem höheren Detailgrad untersucht haben, liegt in dem erhöhten Aufwand, der sich aus der separaten Messung der einzelnen Aktivitäten ergibt (Alam et al., 2014, S. 15; Nurminen et al., 2006, S. 355). Da die Aktivitäten der mechanisierten Aufarbeitung in nur wenigen Studien so detailliert gegliedert wurden, widmet sich die letzte Forschungshypothese den Unterschieden in der Struktur der Kostentreiber zwischen den Aktivitätsmodellen und dem gesamten Prozessmodell:

H6: Die separate Analyse der Aktivitäten offenbart signifikante Kostentreiber, die eine gemeinsame Analyse in Form eines Gesamtmodells des Aufarbeitungsprozesses nicht offenbart hätte. 


\section{Material und Methoden}

In Kapitel 6 werden Methoden und Modelle sowie das dazugehörige Material zur Beantwortung der forschungsleitenden Hypothesen vorgestellt. Der erste Abschnitt gibt eine Übersicht über die untersuchten Versuchsflächen und die eingesetzten Harvestersysteme. Im zweiten Teil erfolgt eine Beschreibung des Prozessmodells der mechanisierten $\mathrm{Bu}-$ chenaufarbeitung, welches die Grundlage zur Auswertung der Zeitstudie bildet. Im Anschluss daran werden das Aufnahmedesign und die berücksichtigten Variablen dargestellt sowie die statistischen Methoden dieser Dissertation näher beleuchtet. Den Abschluss bildet eine genauere Beschreibung der Stichprobe, die aus der Aufnahme der Versuchsflächen hervorgegangen ist.

\subsection{Versuchsflächen und Untersuchungsmaschinen}

Im Rahmen der vorliegenden Arbeit wurden auf Flächen von HessenForst und den Niedersächsischen Landesforsten (NLF) vier Versuchsflächen ausgewiesen und auf diesen eine Bestandesvollaufnahme durchgeführt. Eine weitere Versuchsfläche konnte aus den Rohdaten der Arbeit von Kleinschmit (2015) gewonnen werden. Da die Forschungsschwerpunkte und die Untersuchungsmethodik beider Arbeiten jedoch auseinandergehen, decken sich die Variablensets nicht vollständig. Die Daten können aber ergänzend in Teilen der Regressionen einbezogen werden. Eine Übersicht über die fünf Versuchsflächen ist in Tabelle 4 dargestellt. Eine zusätzlich sechste Versuchsfläche, die im niedersächsischen Forstamt Münden (Revier Bursfelde) stehend aufgenommen wurde (320 Einzelbäume), konnte aufgrund der Sturmereignisse im Frühjahr 2018 und deren Folgen nicht aufgearbeitet werden. Die Daten können aber teilweise genutzt werden, um statistische Zusammenhänge zwischen verschiedenen Baumvariablen über eine größere Stichprobe zu beschreiben. 
Tabelle 4: Beschreibung der aufgenommenen Versuchsflächen. Teile der Werte entstammen den Forsteinrichtungsunterlagen der jeweiligen Landesforsten.

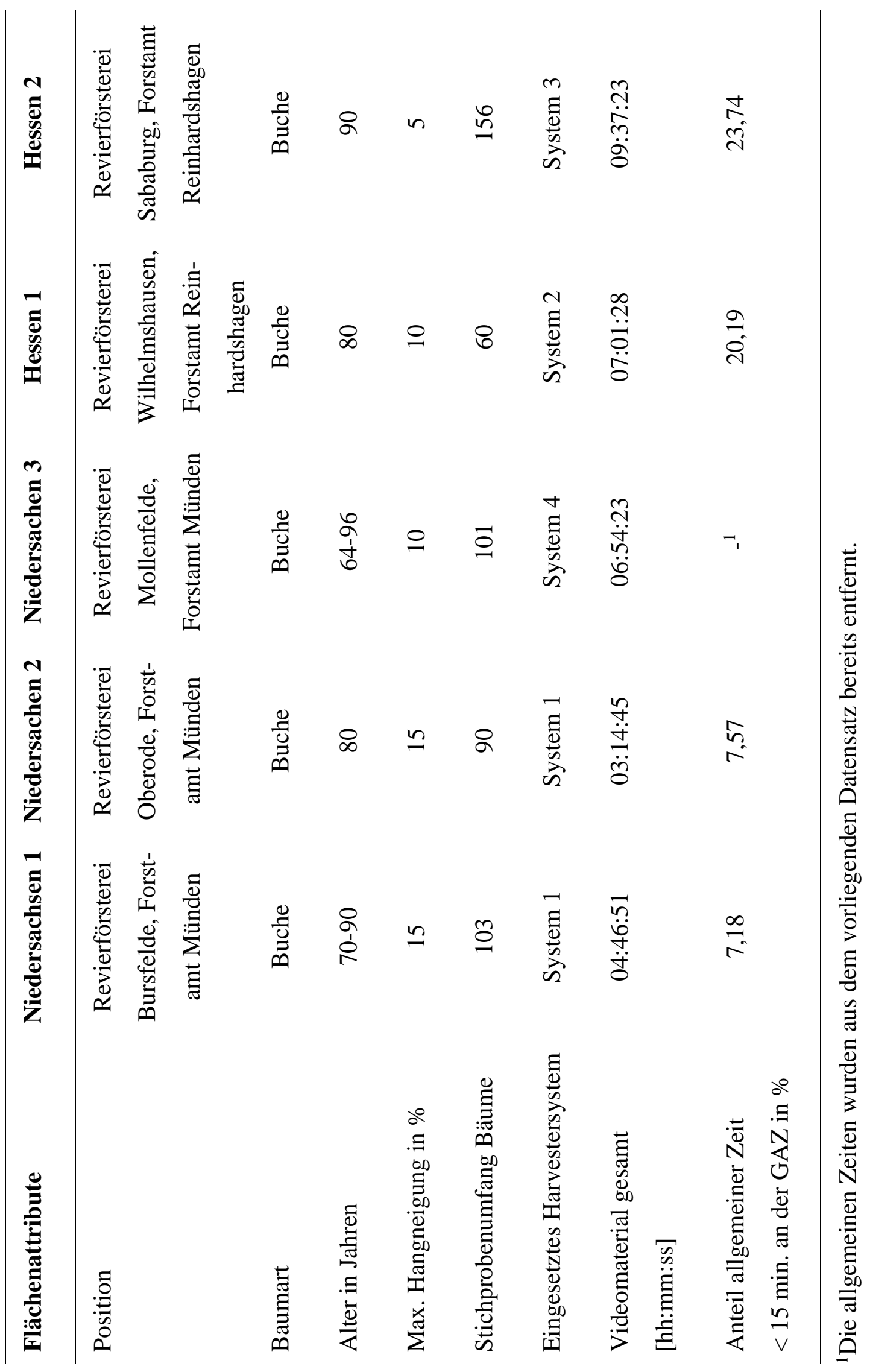


Eine Besonderheit dieser Dissertation ist die Analyse des Aufarbeitungsprozesses auf Einzelbaumebene. Daher ist eine tiefergehende Beschreibung der Versuchsflächen auf Bestandesebene nicht notwendig. Jeder aufgenommene Einzelbaum in den Versuchsflächen stellt einen Teil der Stichprobe dar. Eine genauere Beschreibung der Stichprobe, welche aus den Versuchsflächen hervorgeht, erfolgt in Kapitel 6.4.

Bei den im Rahmen dieser Arbeit untersuchten Maschinen handelt es ausschließlich um Radharvester der großen Leistungsklasse (vgl. Forbrig, 2000, S. 98). Zudem erlauben alle Aggregate eine Fällung von Bäumen über $60 \mathrm{~cm} .{ }^{41}$

Tabelle 5: Übersicht über die genutzten Harvestersysteme zur Aufarbeitung in den Versuchsflächen.

\begin{tabular}{lcccc}
\hline Ausstattung & System 1 & System 2 & System 3 & System 4 \\
\hline Marke und & Ponsse & Ponsse Ergo & Rottne H-20 & John Deere \\
Model & Scorpion & $(8 \mathrm{w})$ & & 1270E IT4 \\
& King & & & \\
Leistung [kW] & 210 & 210 & 187 & 170 \\
Aggregat & H7 & H6 & EGS 700 & Waratah \\
& & & & H480C \\
Vorschubgeschwindigkeit & 5 & 6 & 4 & 4,2 \\
[m/s] & & & & \\
Max. Öffnung [mm] & 650 & 600 & 750 & 680 \\
Krantyp & C50 & C5 & RK200 & CH7 \\
Kranreichweite [m] & 10 & 10 & 10 & 10 \\
Kranhubmoment [kNm] & 252 & 248 & 254 & 197 \\
Mess- und Steuerungssys- & Opti4G & Opti4G & Rottne D4 / & TimberRite \\
tem & & & Rottne D5 & \\
\hline
\end{tabular}

\footnotetext{
${ }^{41}$ Der in Tabelle 5 dargestellte maximale Öffnungsdurchmesser ist nicht gleichzusetzten mit dem maximalen Fälldurchmesser, sondern beschreibt den Durchmesser, bei dem die Vorschubwalzen noch aktiv agieren können.
} 
Jedes Harvestersystem besteht aus der dargestellten Maschine und einem festen Fahrer. Bei allen Fahrern handelt es sich um private Lohnunternehmer, die seit mindestens zehn Jahren in der mechanisierten Holzernte aktiv sind. Die genutzten Harvester waren keine bereitgestellten Regiemaschinen, sondern der jeweilig dauerhaft genutzte Harvester des Maschinenführers (mind. 3 Jahre). Die Fahrer bekamen neben den Angaben im Arbeitsauftrag des Revierleiters (Anzahl der Sorten, sortenspezifische Längen-, Durchmesserund Qualitätseinschränkungen) keine zusätzlichen Anweisungen. Die Maßnahmen fanden systemübergreifend zwischen 08:00 und 18:00 statt. Diese Voraussetzungen sollten sicherstellen, dass der Prozess der mechanisierten Buchenaufarbeitung praxisnahen Bedingungen unterlag.

Eine Ausnahme besteht diesbezüglich in der Versuchsfläche von Kleinschmit (2015). Der Fahrer wurde hier explizit angewiesen, die Abschnitte bei der Aufarbeitung nicht wie im Normalfall sortimentsweise abzulegen, sondern breit auszulegen, um eine spätere Vermessung durch den Aufnahmetrupp zu erleichtern. Die Zeitmessungen von Kleinschmit (2015) für die Aktivitäten innerhalb des Sortimentszyklus werden daher nicht mit in die Analyse eingeschlossen.

\subsection{Aktivitäten der mechanisierten Buchenaufarbeitung}

Ein zentrales Ziel dieser Arbeit bestand darin, die Aktivitäten des Prozesses der mechanisierten Buchenaufarbeitung detailliert zu quantifizieren (H1). Aus diesem Grund wurde im Rahmen von Voruntersuchungen ein Prozessmodell mit einem hohen Detailgrad entwickelt, welches den Prozess auf Aktivitätsebene modelhalft beschreibt (Abbildung 15). Die morphologischen Besonderheiten der Buche bedingen, dass bei der Aufarbeitung eines Baumes teilweise mehrere Stränge (Kapitel 4.1) in einem gesonderten Sortimentszyklus aufgearbeitet werden müssen. Ist ein Strang aufgearbeitet, wird der Kronenrest abgelegt, umgegriffen und der nächste Strang aufgearbeitet. Dies geschieht aber nur optional und spiegelt nicht den obligatorischen Aufarbeitungsweg wider. Hinzu kommen Verzweigungen, bei denen das Harvesteraggregat nicht mehr in der Lage ist, allein durch den Vorschub den abgehenden Ast an den Entastungsmessern abzuschlagen. Das Aggregat muss sich vom Stamm lösen und umgreifen, um den Baum weiter aufarbeiten zu können (Kleinschmit, 2015, S. 49; Schorr, 2000, S. 521). 


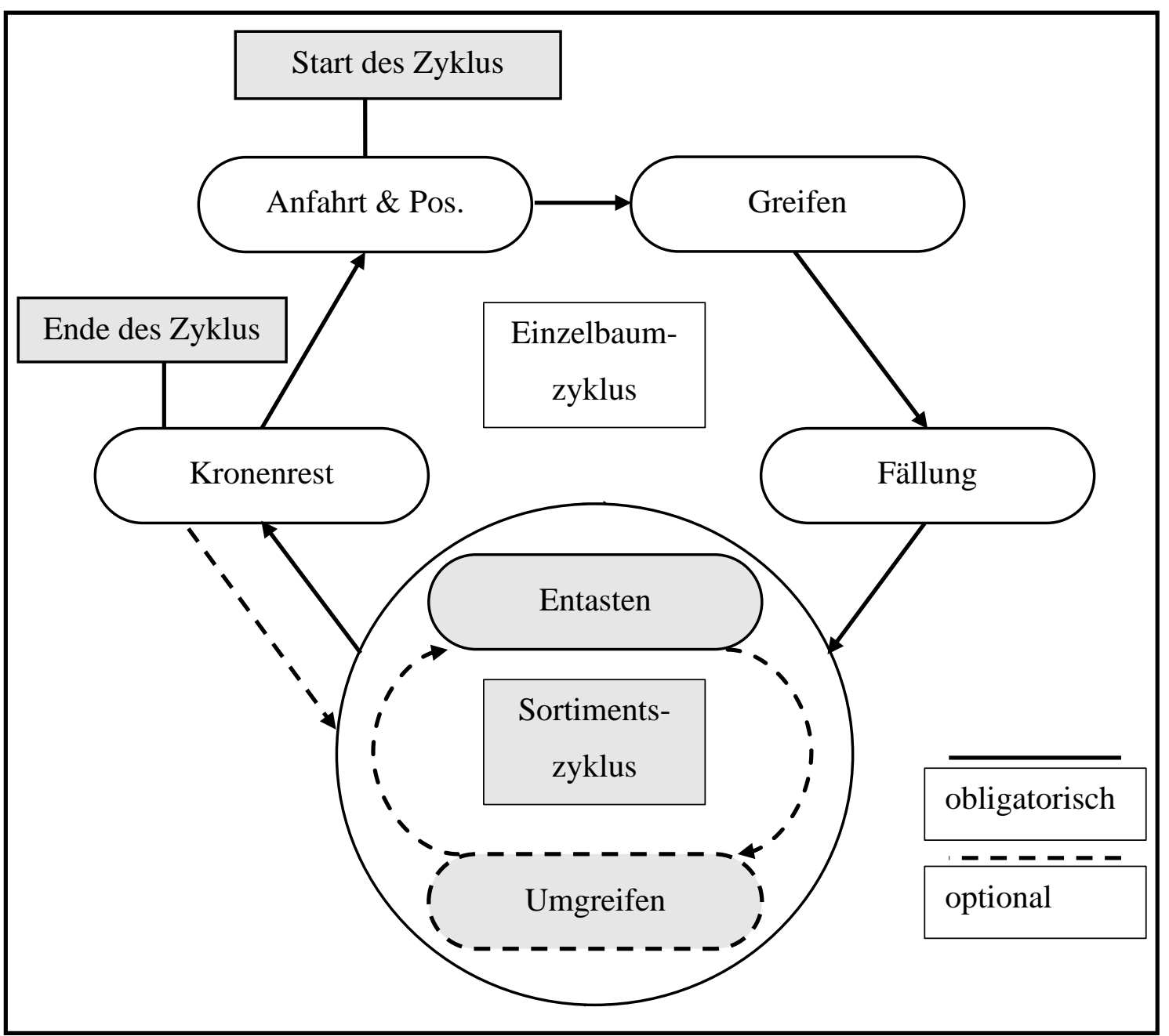

Abbildung 15: Prozessmodell des Einzelbaum- und des Sortimentszyklus für die mechanisierte Buchenaufarbeitung. Eigene Darstellung in Anlehnung an Alam et al. (2014, S. 17).

Ähnlich wie in anderen Arbeiten, wird in diesem Modell auch nicht zwischen „Fahren“ und „Positionieren“ unterschieden (u. a. Alam et al., 2014, S. 17; Purfürst, 2009, S. 52). Diese beiden Aktivitäten überlappen sich sehr stark und können anhand von Videomaterial kaum getrennt werden. Eine Beschreibung der Aktivitäten und der jeweiligen Startund Endzeitpunkte bietet Tabelle 6. Bei simultan ablaufenden Aktivitäten wurde immer die nachfolgende Aktivität priorisiert. So wurde beispielsweise der Kranarm bereits bewegt, obwohl die Anfahrt und Positionierung noch nicht abgeschlossen wurde - diese simultane Zeit wurde ausschließlich dem Greifen zugeordnet. 
Tabelle 6: Beschreibung der Aktivitäten und deren definierte Start- und Endzeitpunkte innerhalb des Prozesses der mechanisierten Buchenaufarbeitung.

\begin{tabular}{ll}
\hline Aktivität & Beschreibung und Trennpunkte der Aktivität \\
\hline Anfahrt und & Fahrt zum und Positionierung am Baum. Beginnt mit dem Ab- \\
Positionierung & schluss des letzten Arbeitsschrittes am zuletzt entnommenen \\
& Baum. Endet mit der Bewegung des Kranarmes, um den nächs- \\
& ten Entnahmebaum zu greifen. \\
Greifen & Folgt auf die Anfahrt und Positionierung. Endet mit dem Schlie- \\
Ben des Aggregats um den Baum. \\
Folgt auf das Greifen. Endet mit dem Anfahren der Aggregat- \\
walzen zur Aufarbeitung des ersten Abschnittes. \\
Beginnt mit dem Anfahren der Aggregatwalzen am Baum- \\
stamm. Endet beim Einklappen der Säge nach dem Trenn- \\
schnitt. \\
Beginnt mit dem Öffnen der Aggregatwalzen innerhalb der Auf- \\
arbeitung. Endet mit dem Schließen der Aggregatwalzen zur \\
Aufarbeitung des nächsten Abschnittes. \\
Beschreibt das Ablegen des letzten Baumstückes, bei dem kein \\
Abschnitt mehr ausgehalten wird. Endet mit dem Öffnen der \\
Aggregatwalzen beim Ablegen oder Fallenlassen des Kronen- \\
restes. \\
Jede Unterbrechung der aufgeführten Aktivitäten. Die Ursache \\
der Unterbrechung (persönlich oder sachlich) wurde vermerkt. \\
\hline
\end{tabular}

Innerhalb der Pretest-Auswertung traten zusätzliche Aktivitäten während des eigentlichen Prozessabschnittes auf. So war es beispielsweise notwendig, dass innerhalb der Fällung ein Säuberungsschnitt durchgeführt werden musste, um zum einen die Messsysteme zu nullen und zum anderen den Waldbart zu entfernen. Aus diesem Grund können viele der Aktivitäten in einem sauberen (N-Verlauf) und in einem gestörten Verlauf (Z-Verlauf) durchgeführt werden. Diese zusätzlichen Aktivitäten traten nur optional auf und sind kein Bestandteil des obligatorischen Aktivitätsinhaltes. Eine Übersicht über alle dokumentierten Zusatzaktivitäten gibt Tabelle 7. 
Tabelle 7: Übersicht über mögliche auftretende zusätzliche Aktivitäten innerhalb der mechanisierten Buchenaufarbeitung.

\begin{tabular}{|c|c|c|}
\hline Aktivität & Zusatzaktivität & Beschreibung \\
\hline \multirow[t]{5}{*}{ Greifen } & Unterwuchs (U) & Das Entfernen von störendem Unterwuchs inner- \\
\hline & & halb des Greifvorganges. \\
\hline & Kranbewegung (K) & Das Aggregat bewegt sich nicht direkt zum Ent- \\
\hline & & nahmebaum, sondern wird zwischendurch wieder \\
\hline & & zurückgezogen (Ursache unbekannt). \\
\hline \multirow[t]{5}{*}{ Fällung } & Loslassen (L) & Der Stamm muss innerhalb des Fällvorganges \\
\hline & & losgelassen und wiederergriffen werden. \\
\hline & Säuberungsschnitt (S) & Durchführung eines Säuberungsschnittes, um den \\
\hline & & $\begin{array}{l}\text { Waldbart zu entfernen und das Messsystem zu } \\
\text { nullen. }\end{array}$ \\
\hline & Ziehen $(\mathrm{Z})$ & $\begin{array}{l}\text { Innerhalb des Fällvorganges wird der Stamm ak- } \\
\text { tiv in eine Richtung gezogen. }\end{array}$ \\
\hline \multirow[t]{4}{*}{ Entasten } & Ast (A) & $\begin{array}{l}\text { Das Aggregat konnte den Abschnitt nicht in ei- } \\
\text { nem Vorschub Entasten und muss sich zusätzlich } \\
\text { entlang des Stammes vor und zurück bewegen. }\end{array}$ \\
\hline & Messen (M) & $\begin{array}{l}\text { Die Abschnittsvermessung ist fehlerhaft. Das Ag- } \\
\text { gregat fährt in Folge ruckartig vor und zurück. }\end{array}$ \\
\hline & Ziehen $(\mathrm{Z})$ & $\begin{array}{l}\text { Innerhalb des Entastens wird der Abschnitt bzw. } \\
\text { der restliche Baum aktiv in eine Richtung gezo- } \\
\text { gen. }\end{array}$ \\
\hline & Säuberungsschnitt (S) & $\begin{array}{l}\text { Innerhalb des Entastens musste ein Säuberungs- } \\
\text { schnitt durchgeführt werden, um das Messsystem } \\
\text { des Aggregates zu nullen. }\end{array}$ \\
\hline $\begin{array}{l}\text { Kronen- } \\
\text { rest }\end{array}$ & Gassenablage $(\mathrm{G})$ & $\begin{array}{l}\text { Der Kronenrest wurde explizit auf der Rücke- } \\
\text { gasse abgelegt, um die Bodenpfleglichkeit zu er- } \\
\text { höhen. }\end{array}$ \\
\hline
\end{tabular}

Diese Zusatzaktivitäten lassen sich nicht einzeln vermessen, da sie sich nicht durch einen klaren Start- und Endzeitpunkt von der eigentlichen Aktivität trennen lassen. Sie stellen eine besondere Herausforderung für die anschließende Regressionsanalyse dar, da sie einer Aktivität zugeordnet werden müssen. Hierzu müssen zusätzliche Regeln definiert 
werden, die bei der Analyse von Nadelholz teilweise nicht notwendig sind. Solche Zusatzaktivitäten wurden bereits in anderen Studien thematisiert und als Erklärung für auftretende Streuungen genutzt, aber nicht statistisch ausgewertet (z. B. Ziehen bei der Fällung und Säuberungsschnitt, Alam et al., 2014, S. 21). In dieser Arbeit wurde das Auftreten sowie die Art und Anzahl der Zusatzzeit vermerkt. So standen diese Informationen für die folgenden Regressionsanalysen zur Verfügung (Tabelle 7).

\subsection{Aufnahmedesign}

Das Aufnahmedesign dieser Dissertation ist eingeteilt in vier Bereiche. Der allgemeine Aufbau der Studie orientierte sich an der Richtlinie von Magagnotti et al. (2012), da sich diese als besonders geeignet für die Aufnahme von forstlichen Arbeitszeitstudien erwiesen hat (Magagnotti et al., 2013, S. 252). Zuerst wurden die Entnahmebäume in den Versuchsflächen stehend vermessen. Im Anschluss daran erfolgten die Videodokumentation und die Zeitstudie der mechanisierten Aufarbeitung. Sobald diese abgeschlossen waren, konnten die aufgearbeiteten Abschnitte vermessen, sortiert und dem jeweiligen Baum zugeordnet werden. Den Abschluss der Aufnahme bildete die statistische Datenanalyse.

\subsubsection{Stehende Aufnahme}

In Vorbereitung der Aufarbeitungsmaßnahmen wurden in einem ersten Schritt die Versuchsflächen nach Maßgabe der Waldbaurichtlinien der NLF bzw. des Hessenforsts durch den jeweiligen Revierleiter im Konzept einer Z-Baum-Auslesedurchforstung ausgezeichnet und die zur Entnahme vorgesehenen Bäume markiert. Im Anschluss daran erfolgte die stehende Aufnahme dieser Entnahmebäume. Eine Übersicht über die erhobenen Variablen und die verwendete Aufnahmemethodik zeigt Tabelle 8. 
Material und Methoden

Tabelle 8: Übersicht über die stehend erhobenen Baumvariablen und das jeweilige Aufnahmeverfahren für alle ausgezeichneten Bäume in den Versuchsflächen

\begin{tabular}{ll}
\hline Baumvariable & Aufnahmeverfahren \\
\hline BHD & 1,3 m Bodenhöhe (ermittelt über Kreuzkluppung) in cm \\
Kronentyp & Einteilung in die drei Kronentypen wipfelschäftig, be- \\
& senförmig und zwieselförmig (Kapitel 4.1) \\
Kronenansatzhöhe & Höhe des ersten Primärastes (Kapitel 4.1) in m \\
Baumhöhe & Höhe der Baumspitze in m \\
Asthöhe & Höhe des ersten Grünastes in m \\
Zwieselhöhe & Höhe eines Nebentriebes mit mind. 75 \% der Stärke des \\
& stärkeren Triebes in m (Börner et al., 2003, S. 276) \\
Qualität & Optische Qualitätseinschätzung des unteren Stammbe- \\
& reiches - Einteilung in eine von sechs Güteklassen \\
& (FVA-BW, 2001, S. 6-7) \\
Entfernung zur Rückegasse & Entfernung des Baumes zur nächstgelegenen Rücke- \\
& gasse - Messung rechtwinklig an der Gassenmitte in m \\
Entfernung zum nächsten & $\begin{array}{l}\text { Entfernung zum nächstgelegenen Entnahmebaum ent- } \\
\text { Baum }\end{array}$ \\
lang der Aufarbeitungsrichtung - Messung an der Gas- \\
senmitte in m
\end{tabular}

Alle Höhen und Entfernungen wurden mittels Forestor-Vertex IV der Firma Haglöf gemessen. Die Einschätzung der Qualität am stehenden Stamm erfolgte nach einer Arbeitsanweisung der Forstlichen Versuchs- und Forschungsanstalt Baden-Württemberg (FVABW), die im Rahmen der Aufnahmen der zweiten Bundeswaldinventur erarbeitet wurde. Die Anweisung ermöglicht die Einschätzung im Rahmen einer Notenskala ${ }^{42}$ und bewertet die untere Stammlänge des Baumes (FVA-BW, 2001, S. 2). Unter Einbeziehung des Kronentyps konnte somit eine umfassende Qualitätseinschätzung des Baumes vorgenommen werden. Zusätzlich zu der Aufnahme der Baumvariablen wurden die Bäume für die spätere Zuordnung bei der Videoauswertung fortlaufend nummeriert. Dazu wurde die jeweilige Nummer von drei Seiten in rund 1,8 m Höhe angesprüht.

\footnotetext{
${ }^{42}$ Die Qualitätseinschätzung erfolgte von 1 = „,beste Qualität“ bis $6=$ „,nicht mehr als Stammholz verwendbar".
} 


\subsubsection{Videodokumentation}

An die stehende Aufnahme schlossen die maschinelle Fällung und Aufarbeitung der Entnahmebäume an. Während der Aufarbeitung herrschten durchweg trockene Wetterbedingungen. Die Arbeitsabläufe des Harvesters wurden mit einer an der Windschutzscheibe der Fahrerkabine installierten Videokamera gefilmt (GoPro HERO5, $170^{\circ}$ Aufnahmeradius). Die Kamera wurde zentral hinter dem Frontbildschirm der Harvesterkabine und damit außerhalb des Sichtfeldes des Fahrers befestigt (Abbildung 16). Die Installation der Kamera erfolgte vor Arbeitsbeginn, filmte dann ununterbrochen die Tätigkeiten des Arbeitstages und wurde am Ende des Arbeitstages wieder abgebaut. Diese Vorkehrung wurden getroffen, um den Fahrer so wenig wie möglich in seinem Arbeitsablauf zu stören und einen Überwachungseffekt zu vermeiden (Eriksson \& Lindroos, 2014, S. 179). Das Videomaterial hatte eine Bildwechselfrequenz von 60 Bildern pro Sekunde und ermöglichte damit eine Zeitmessung mit einer Genauigkeit von 0,017 Sekunden. Das Videomaterial wurde im Anschluss im Rahmen eines Fortschrittszeitverfahrens mit Hilfe des Videoplayers Avidemux (Version 2.6) gesichtet. So konnten die in Kapitel 6.2 definierten Zeiten der Aktivitäten einzeln gestoppt werden. Durch die Nummerierung der Bäume konnten die Zeiten eindeutig mit den Daten der stehenden Aufnahme verknüpft werden.

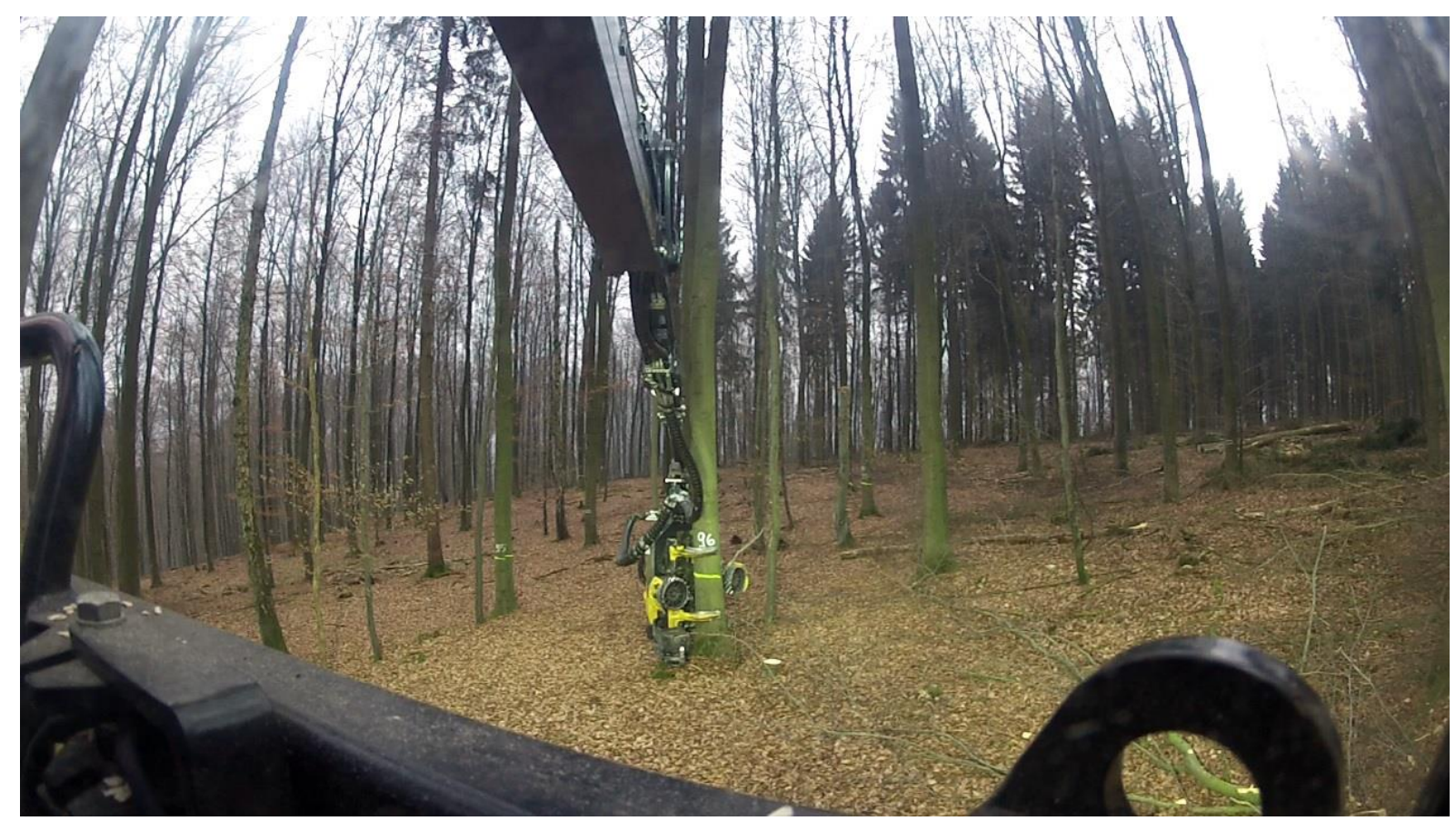

Abbildung 16: Beispielaufnahme aus der Harvesterkabine mit der installieren Videokamera. 


\subsubsection{Liegende Aufnahme}

Nach der Aufarbeitung der Bäume und vor der Holzrückung erfolgte die Aufnahme der liegenden Abschnitte (Abbildung 17). Diese wurden im ersten Schritt RVR-konform sortiert und vermessen. Hierzu wurde der Mittendurchmesser über Kreuz gekluppt und die Länge durch die Nutzung eines Spencer Forstmaßbandes vermessen. Bei angefallenen Stammholzsortimenten wurde zusätzlich die Güte durch eine Qualitätssortierung (RVR) eingeschätzt. Zuletzt wurde die Herkunft der Sortimente (Stamm- oder Kronenbereich) bewertet und alle Abschnitte dem nummerierten Entnahmebaum zugeordnet. Damit waren die Daten aller drei Aufnahmen eindeutig miteinander verknüpft. Alle Durchmesser wurden ohne Rindenabzüge vermessen.

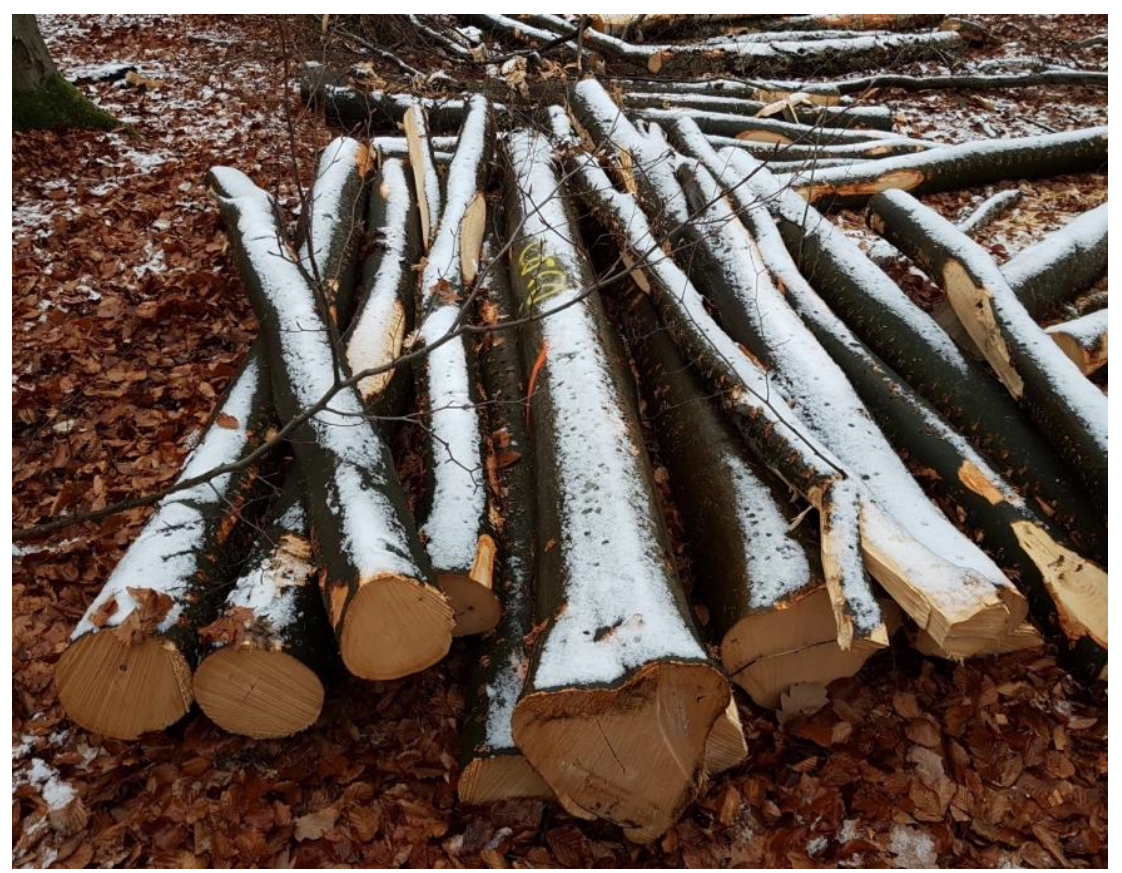

Abbildung 17: Beispielaufnahme eines Raupolters, das bei der mechanisierten Aufarbeitung entsteht.

Insgesamt wurden vier verschiedene Sortimente mit einheitlichen Vorgaben bezüglich der Länge, des Durchmessers und der Qualität ausgehalten. Die Anzahl der Sortimente folgte den Arbeitsanweisungen entsprechend in den jeweiligen Versuchsflächen gesondert. Sie wurde demnach nicht durch das Untersuchungsdesign vorgegeben. Die Aushaltung wurde ebenfalls dokumentiert und es war bekannt, wie viele und welche Sortimente auf einer Fläche ausgehalten wurden. 
Industrieholz (IS) wurde in $3 \mathrm{~m}$ (minimaler Zopf $=7 \mathrm{~cm} \mathrm{~m}$. R.), Schälholz (S) ${ }^{43}$ in 5,4 m und Palette $(\mathrm{PA})^{44}$ in 2,6 m Länge ausgehalten. Das Stammholz $(\mathrm{SH})^{45}$ wurde in fallenden Längen mit mindestens 5,4 $\mathrm{m}$ ausgehalten.

Eine entscheidende Bezugsgröße ist das Volumen der entnommenen Bäume (Kapitel 4.4.2). Im forstlichen Kontext werden hauptsächlich zwei Größen verwendet: Zum einen eine Angabe in Vorratsfestmeter (Vfm), welche das Volumen des stehenden Stammes umfasst und zum anderen in Erntefestmeter (Efm), die dem Vorratsvolumen des Baumes abzüglich anfallender Ernteverluste (Rinde, Äste, etc.) entspricht. Die Berechnung der stehenden Einzelbaumvolumina erfolgte über die Funktion nach Bergel (1973), in welche die Parameter Höhe $=h(\mathrm{~m})$ und Brusthöhendurchmesser $=d(\mathrm{~cm})$ eingehen:

$$
\begin{aligned}
V f m & =\pi * h *\left(\frac{d}{200}\right)^{2} *(0,4039+0,0017335 * h \\
& \left.+\frac{1,1267}{h}-\frac{118,188}{d^{3}}+0,0000042 * d^{2}\right)
\end{aligned}
$$

Formel 7: Einzelbaum-Volumenfunktion (Vfm) für die Baumart Buche nach Bergel (1973).

Als Grundlage für das Stückvolumengesetz gilt aber nicht das stehende, sondern das verkaufsfähige Holzvolumen (Purfürst, 2009, S. 73). Insofern musste das Volumen umgerechnet werden. In vielen praktischen Berechnungen wird oft die Faustzahl von 0,8 für die Umrechnung von Vfm zu Efm (10\% Rindenverlust, 10\% Ernteverlust) genutzt (Elsasser et al., 2020, S. 19; Schluhe et al., 2018, S. 71). Da die Anwendung eines einfachen Umrechnungskoeffizienten jedoch einen proportionalen Anstieg des Erntevolumens (Efm) mit steigendem Baumvolumen (Vfm) voraussetzt und dieser Zusammenhang nicht mit den Beobachtungen dieser Arbeit korrespondierte, wurde das reell gemessene Erntevolumen - also die Summe aller gemessenen Abschnitte je Baum - als Erntevolumen des Einzelbaumes definiert. Abbildung 18 zeigt das theoretische Vorratsvolumen nach Bergel (1973) über dem reell gemessenen Baumvolumen (ohne Rindenabzüge, Efm m. R.). Es zeigte sich, dass die geschätzten Erntevolumina nach Bergel (Faktor 0,8; schwarz gestrichelte Linie) zwar im Durchschnitt die reell gemessenen Erntevolumina gut beschreiben.

\footnotetext{
${ }^{43}$ Zopfdurchmesser mindestens $26 \mathrm{~cm}$ o. R. - keine Zugabe.

${ }^{44}$ Zopfdurchmesser mindestens $33 \mathrm{~cm} \mathrm{~m}$. R. - Zugabe $10 \mathrm{~cm}$.

45 Zopfdurchmesser mindestens $35 \mathrm{~cm}$ o. R. - Zugabe $20 \mathrm{~cm}$.
} 
Jedoch wäre die erhebliche Datenschwankung durch Anwendung eines einfachen Umrechnungskoeffizienten zur Erntevolumenschätzung abgeschnitten. Dadurch wären interessante Informationen für die spätere Analyse verloren gegangen. Die Schwankungen oberhalb der Winkelhalbierenden sind in der Funktion von Bergel (1973) begründet, die Parameter wie den Kronentyp, welche einen erheblichen Einfluss auf das nutzbare Baumvolumen haben, nicht mit einbeziehen.

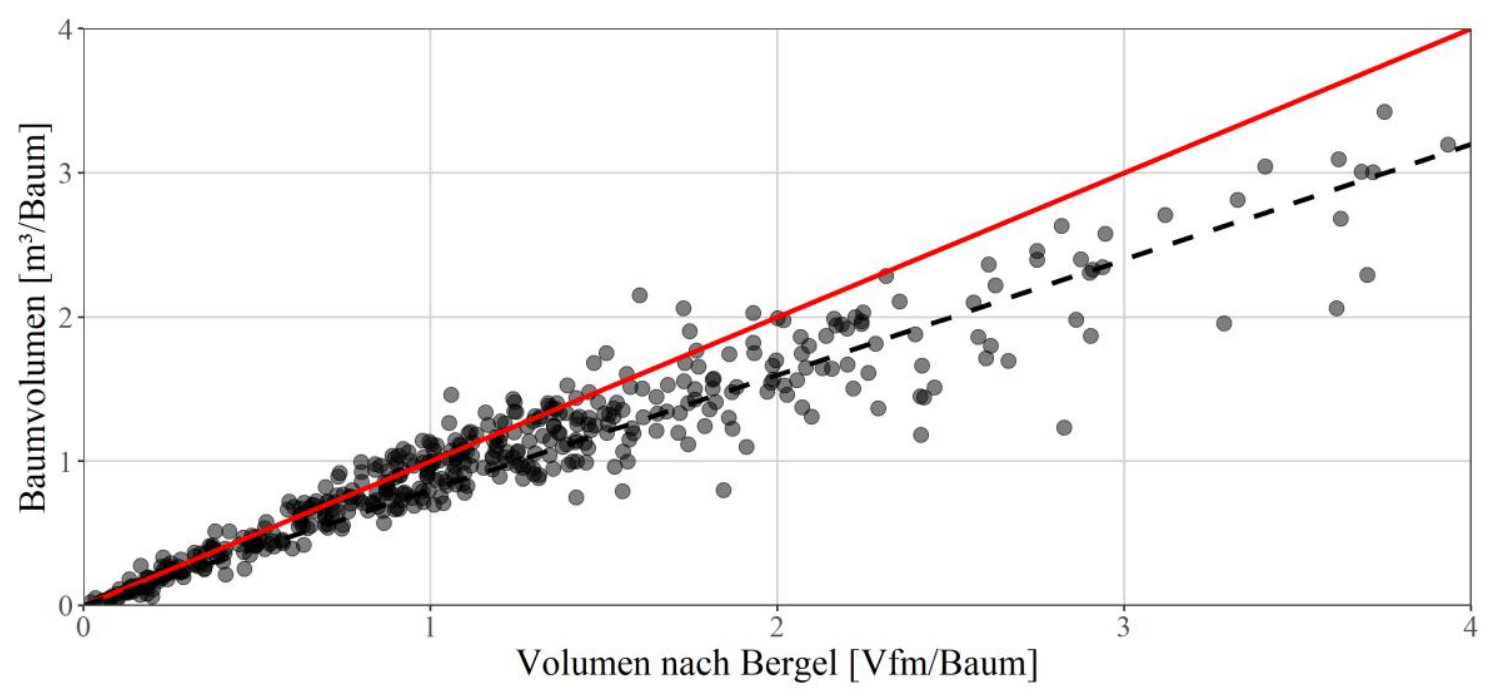

Abbildung 18: Vergleich des Einzelbaumvolumen über die Funktion nach Bergel (1973) mit der Summe der aufgenommenen Abschnitte (m. R.) im Wald (=Baumvolumen). Die rote durchgezogene Linie stellt eine Relation $=1$ und die schwarze gestrichelte Linie eine Relation $=0,8$ dar; $N=254$.

Um dem Detailgrad dieser Untersuchung gerecht zu werden, wurde daher nicht das Vorratsvolumen über die Funktion nach Bergel (1973) geschätzt, sondern stattdessen das reell aufgearbeitete Volumen über die Summe der aufgearbeiteten Abschnitte als Baumvolumen in dieser Arbeit genutzt (mit Rinde). Es kann also als Erntefestmetermaß ohne Berücksichtigung von Rindenabzügen verstanden werden. Alle Grafiken und Ergebnisse, die diesem Kapitel folgen und das Baumvolumen als Koeffizienten beinhalten, beziehen sich auf das hergeleitete Baumvolumen über die Summe der aufgearbeiteten Abschnitte pro Baum in $\mathrm{m}^{3}$. 


\subsubsection{Datenanalytische Methodik}

Die Vorgehensweise und die Umsetzung von statistischen Verfahren wurden in der Literatur bereits ausgiebig beschrieben, sowohl für einen allgemeinen Einsatzbereich (u. a. Fahrmeir et al., 2013; Sachs, 1997) als auch in spezielleren Kontexten wie der Auswertung von forstlichen Zeitstudien (u. a. Jacke, 1980; Purfürst, 2009). Aus diesem Grund soll an dieser Stelle auf diesen Aspekt nicht näher eingegangen werden, sondern vielmehr der jeweilige Einsatzbereich und die Besonderheiten verschiedener Verfahren innerhalb dieser Dissertation aufgezeigt werden.

Dadurch, dass die Arbeitsschritte nicht experimentell vorgegeben wurden, sondern das Versuchsdesign sich vielmehr aus den Arbeitsanweisungen ergab, mussten einige Besonderheiten berücksichtigt werden. So konnten beispielsweise nicht alle Abschnitte zweifelsfrei zugeordnet werden, sodass Datenlücken bei Längen und Mittendurchmessern entstanden (die Aufarbeitungszeiten waren dennoch alle aus dem Video bekannt). Dieser Umstand führte zu gelegentlichen Lücken in den Datenzeilen, was sich nachteilig auf die statistische Auswertung auswirken könnte. Wenn durch fehlende Einzelwerte die gesamte Datenzeile (Beobachtung) verworfen wird, reduziert sich die Anzahl der Beobachtungen (Cremer, 2008, S. 36). Um zu vermeiden, dass gesamte Zeilen verworfen werden müssen, nur weil einzelne Werte fehlen, verfolgte die Datenanalyse (mittels der Software $\mathrm{R}$, Version 4.0.2) daher einen zweistufigen Ansatz.

In einem ersten Schritt wurden fehlende Variablenausprägungen durch Regressionen ausgeglichen. Die Herausforderung war dabei, Datenlücken zu ergänzen, ohne dem Datensatz zusätzliche Informationen hinzuzufügen und damit in der Folge möglicherweise zu verzerren. Deshalb war es bei der Datenergänzung ausgesprochen wichtig, die exakte durchschnittliche Ausprägung zu finden und einzusetzen. Damit sollte sichergestellt werden, dass eine ergänzte Datenlücke in der späteren Datenanalyse keinen Einfluss auf das Ergebnis haben konnte. Der Fokus lag hier auf der bestmöglichen Erklärung der abhängigen Variablen mit maximaler Genauigkeit. Je nach Skalenniveau der Variable kamen dabei unterschiedliche Verfahren zum Einsatz. Innerhalb der Untersuchung wurden sowohl nominal (z. B. Kronentyp), ordinal (z. B. Qualität) sowie metrisch (z. B. BHD) skalierte Variablen erhoben. Zum Ausgleich metrischer Variablen wurden Spline-Regressionsmodelle genutzt. Nominal skalierte Variablen wurden entweder über ein generalisiertes Spline-Modell mit binominaler Verteilung oder über ein generalisiertes lineares 
Modell mit binominaler Verteilung ausgeglichen. Spline-Funktionen bieten unter den statistischen Standardverfahren die größtmögliche Flexibilität bei der Funktionsschätzung (Xue \& Liang, 2009, S. 27). Sie gelten deshalb als Standard bei der Datenergänzung (Faes et al., 2011, S. 959-960; Kashani \& Dinpashoh, 2012, S. 59-60). Alle genutzten Modelle und die Ergebnisse befinden sich in der Modelldatenbank der Abteilung Forstökonomie der Georg-August-Universität Göttingen (Link: https://gitlab.gwdg.de/forest_economics_goettingen). ${ }^{46}$ So kann die Datenergänzung transparent nachvollzogen werden. Eine Übersicht über die Dateien und deren Inhalt zeigt die Tabelle im Anhang 1.

Nach der Datenergänzung wurden lineare Regressionsmodelle zu Überprüfung der Hypothesen formuliert. Dabei stand nicht nur die Prüfung der Hypothesen selbst im Fokus, sondern es sollten auch in der Praxis nutzbare Modelle erzeugt werden, um die Erkenntnisse dieser Arbeit nicht nur theoretisch nutzbar zu machen. Aus diesem Grund wurden für die Modelle der jeweiligen Prozessaktivitäten (metrisch) keine Spline-Funktionen, sondern lineare Regressionsmodelle berechnet. Die abhängigen Variablen wurden teilweise transformiert (Kapitel 3.3.1). Dieses Vorgehen ließ sich mit dem theoretischen Rahmen des Stückvolumengesetzes (Hilf, 1928) vereinbaren. So konnten die angepassten Modelle zu Stücksatz- und Produktivitätsmodellen umformuliert werden. Um die Genauigkeit der einzelnen Aktivitätsmodelle nicht zu verlieren, wurde für die Erklärung des Gesamtzeitverbrauchs der mechanisierten Buchenaufarbeitung kein neues Gesamtmodell erstellt, sondern die einzelnen Modelle aufaddiert. Die Besonderheit dieses Vorgehens wird in Kapitel 7.2 noch einmal ausführlich beleuchtet. Hinzu kommen geordnete logistische Regressionen (ordinale Logit-Modelle), welche die Abhängigkeit von ordinal-skalierten Variablen, wie beispielsweise der Qualität, in Abhängigkeit von anderen Baumparametern beschreiben. Dazu wurde die geordnete Variante dieses Modells genutzt, da diese bei geordneten ordinal-skalierten Klassen robuster schätzt (Fahrmeir et al., 2013, S. 334-337). Wie beim einfachen logistischen Modell wurden auch hier Eintrittswahrscheinlichkeiten für jede Ausprägung der abhängigen Variablen berechnet - im Unterschied zum einfachen logistischen Modell allerdings nicht für eine binäre, sondern für eine geordnete ordinal-skalierte Variable. In dieser Arbeit wurde ein Teil der Variablen Dummy-codiert (z. B. Abschnittsherkunft aus dem Stamm- oder Kronenbereich). Dies war notwendig, um den Einfluss einer nominal-skalierten Variablen auf eine metrische

\footnotetext{
${ }^{46}$ Maximal wurden 4\% der Beobachtungen innerhalb einer Variablen ausgeglichen.
} 
Variable erklären zu können. Die Variable hat anschließend nur noch die Ausprägungen 1 (Ausprägung liegt vor) und 0 (Ausprägung liegt nicht vor). Alle Regression wurden nach einem einheitlichen Ablauf durchgeführt (Purfürst, 2009, S. 86):

- „Bestimmung der theoretisch denkbaren Variablen (unabhängige-, abhängige-, Dummy-Variablen)

- Wahl des Modells (Ggf. Transformation der Variablen)

- Bestimmung der Parameter des Modells (eigentliche Regressionsanalyse)

- Modellselektion anhand einer AIC-gesteuerten, rückwärtsgerichteten Variablenelimination

- Überprüfung des Modells (Vergleich mit Klassenmittelwerten, P-P-Diagramme)

- Bewertung der Regressionsanalyse (Güte der Anpassung, Korrelation)

- Überprüfung der Modellvoraussetzungen (Residualanalyse)“

Die Bewertung der Güte und die damit verbundene Auswahl eines geeigneten Modells orientierte sich an verschiedenen Informationskriterien. Das Akaike-Informationskriterium $(A I C)$ ist eines der am häufigsten verwendeten Kriterien, um die Modellauswahl im Rahmen einer Likelihood-basierten Interferenz zu bewerten (Fahrmeir et al., 2013, S. 148). Das Kriterium ist dabei negativ orientiert - je geringer der Wert, desto bevorzugter ist das Modell. Die beiden Faktoren Anpassungsgüte und Modellkomplexität beschreiben in der Berechnung einen antagonistischen Einfluss auf den $A I C$-Wert. Mit steigender Anpassungsgüte, die über die Likelihood-Funktion berechnet wird, sinkt der AIC-Wert eines Modells. Eine zu hohe Modellkomplexität, die über die Parameteranzahl in die Berechnung mit eingeht, lässt ihn wiederum steigen. Der $A I C$-Wert wird in dieser Arbeit als Maßgabe für die Qualität der Modelle genutzt.

\subsection{Stichprobe}

Die Stichprobe umfasst nach Bereinigung des Datensatzes insgesamt $N=464$ gültige Einzelbaumdatensätze, die in $N=3.044$ Abschnitte aufgearbeitet wurden. Damit konnten $91 \% \operatorname{der} N=510$ stehend aufgenommen Bäume für die Auswertung nutzbar gemacht werden. Die fehlenden Bäume wurden hauptsächlich nicht aufgearbeitet und daher komplett ausgeschlossen. Eine genaue Übersicht über die Stichprobe und die wichtigsten Variablenausprägungen (getrennt nach Versuchsfläche) zeigt Tabelle 9. 
Material und Methoden

Tabelle 9: Deskriptive Statistik der erhobenen Bäume. Min $=$ Minimum; $U \cdot S D=$ Arithmetischer Mittelwert - Standardabweichung; $M=$ Arithmetischer Mittelwert; O.SD. = Arithmetischer Mittelwert + Standardabweichung; Max = Maximum.

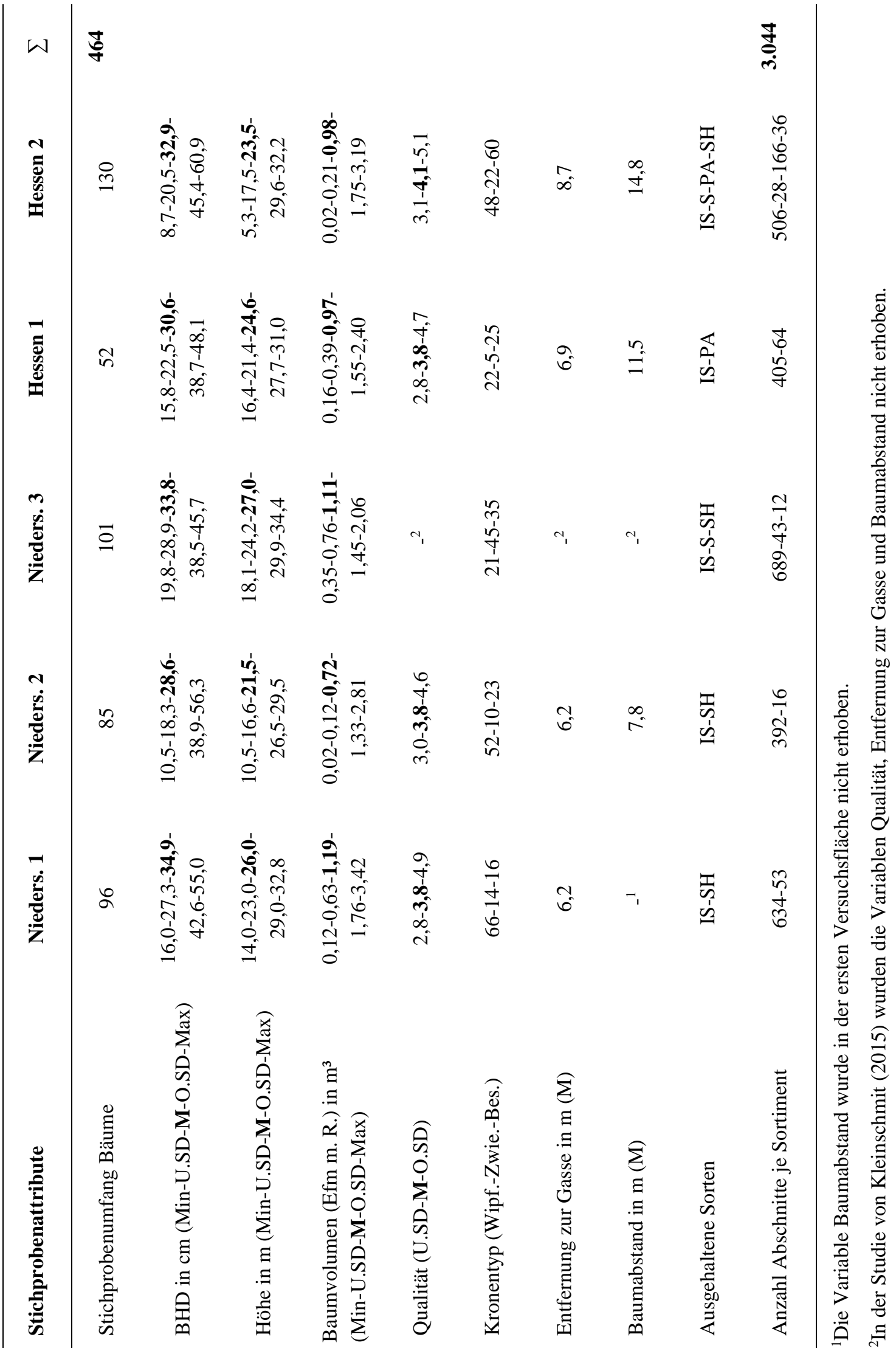




\section{Ergebnisse}

Die Ergebnisdarstellung orientiert sich an der Überprüfung der Forschungshypothesen aus Kapitel 5 und ist eingeteilt in zwei Bereiche. Im ersten Teil erfolgt die Modellierung der einzelnen Aktivitäten. Jede Aktivität wird dabei einzeln im Rahmen eines analytischen Produktivitätsmodells beschrieben. Hierzu werden je Aktivität lineare Modelle geschätzt (H1). Darüber hinaus werden auf Basis der einzelnen Modelle die Hypothesen H2 bis $\mathrm{H} 4$ beantwortet. Im Anschluss wird ein Gesamtmodell eines forstlichen Produktivitätsmodells berechnet und die aufgestellten Hypothesen (H2-H6) vor diesem Hintergrund noch einmal betrachtet. Zusätzlich erfolgt eine Sensitivitätsanalyse des Gesamtmodells mit einer Anwendung auf modellierten Flächen, die auf den Ergebnissen der dritten Bundeswaldinventur beruhen, um die Erkenntnisse der Modelle beispielhaft auf die Fläche zu extrapolieren.

\subsection{Modellierung der Aktivitäten}

Um einen ersten Überblick über die Größenordnung der Zeitverbräuche der Aktivitäten (Abbildung 15) zu bekommen, werden zunächst mittlere Zeitverbräuche berechnet. In der Abbildung 19 sind alle Einzelbäume in Volumengruppen eingeteilt; weiterhin wird der mittlere relative und absolute Zeitverbrauch pro Aktivität und Volumengruppe darin aufgezeichnet - eine Häufigkeitsverteilung innerhalb der Volumenklassen zeigt Anhang 2. Im absoluten Gesamtzeitverbrauch lässt sich ein annähernd linearer Anstieg erkennen, welcher den erwarteten Einfluss des Baumvolumens auf die Produktivität bereits erahnen lässt. Die relativen Anteile der Aktivitäten verändern sich dabei stark über dem Baumvolumen hinweg und es wird ersichtlich, dass der Volumentrend des Baumvolumens nicht auf alle Aktivitäten gleich wirkt. Während die Anfahrt und Positionierung bei Bäumen mit einem Volumen unter $1,25 \mathrm{~m}^{3}$ beispielsweise fast die Hälfte der Aufarbeitungszeit beansprucht, sinkt dieser Anteil mit steigendem Einzelbaumvolumen deutlich, da sich der absolute Wert über dem Baumvolumen kaum verändert. Dafür steigt die benötigt Arbeitszeit für die Aktivitäten der direkten Holzaufarbeitung (Fällung, Entasten und Umgreifen) deutlich an. 

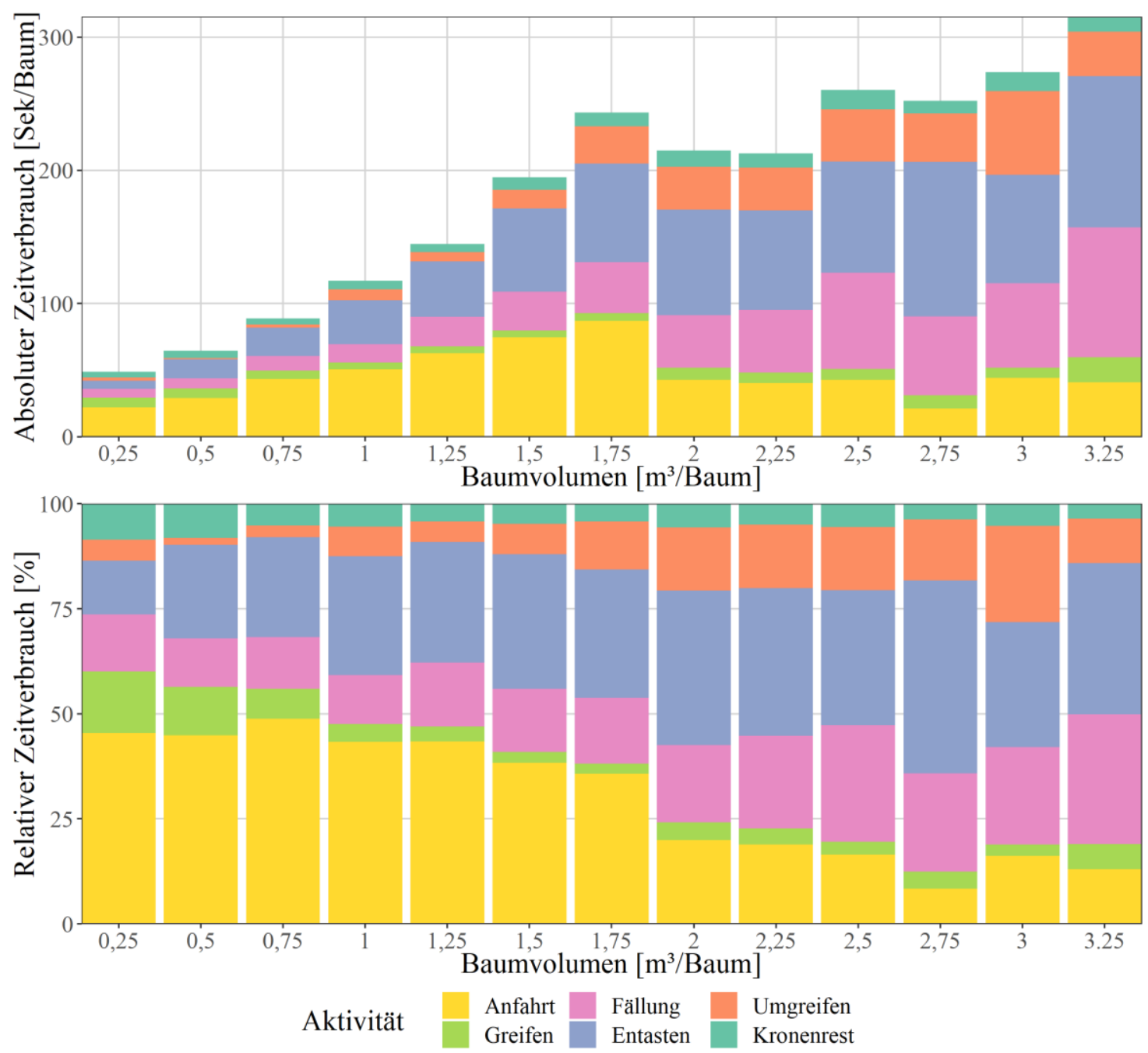

Abbildung 19: Absoluter und relativer Zeitverbrauch bei der Aufarbeitung der beobachteten Einzelbäume in Abhängigkeit zum Baumvolumen (0,25 $\mathrm{m}^{3}$-Stufenbreite) auf Basis der Aktivitäten. Der Gruppenwert des Volumens gibt die obere Grenze des Volumenbereiches an; $N=254$.

Einen etwas tieferen Einstieg in die Zusammenhänge der Parameter bietet die Korrelationsmatrix. Hierbei können jedoch nur metrisch-skalierte Baumvariablen berücksichtigt werden (Abbildung 20). Der unterschiedlich starke lineare Einfluss des Baumvolumens auf die Zeitverbräuche der Aktivitäten sowie auf weitere Baumkovariablen wird hier bereits eindrücklich angedeutet.

Wie zu erwarten, stehen nicht alle Variablen gleichermaßen in einem signifikanten linearen Zusammenhang mit den Zeitverbräuchen der Aktivitäten. So lassen sich bereits in diesem Analyseschritt spezielle aktivitätsabhängige Kostentreiber ausmachen - beispielsweise besteht ein besonders starker linearer Zusammenhang zwischen der Anzahl an Umgreifvorgängen pro Baum und der absoluten Zeit, die für das Umgreifen benötigt 
wird. Die Aussagekraft der Korrelationsmatrix muss aber etwas relativiert werden, da wichtige nominal- und ordinal-skalierte Variablen wie der Kronentyp und die Qualität nicht einbezogen werden können und nur lineare Zusammenhänge analysiert werden. Des Weiteren können je nur Zweierpaare betrachtet werden, obwohl multivariable Zusammenhänge zu vermuten sind.

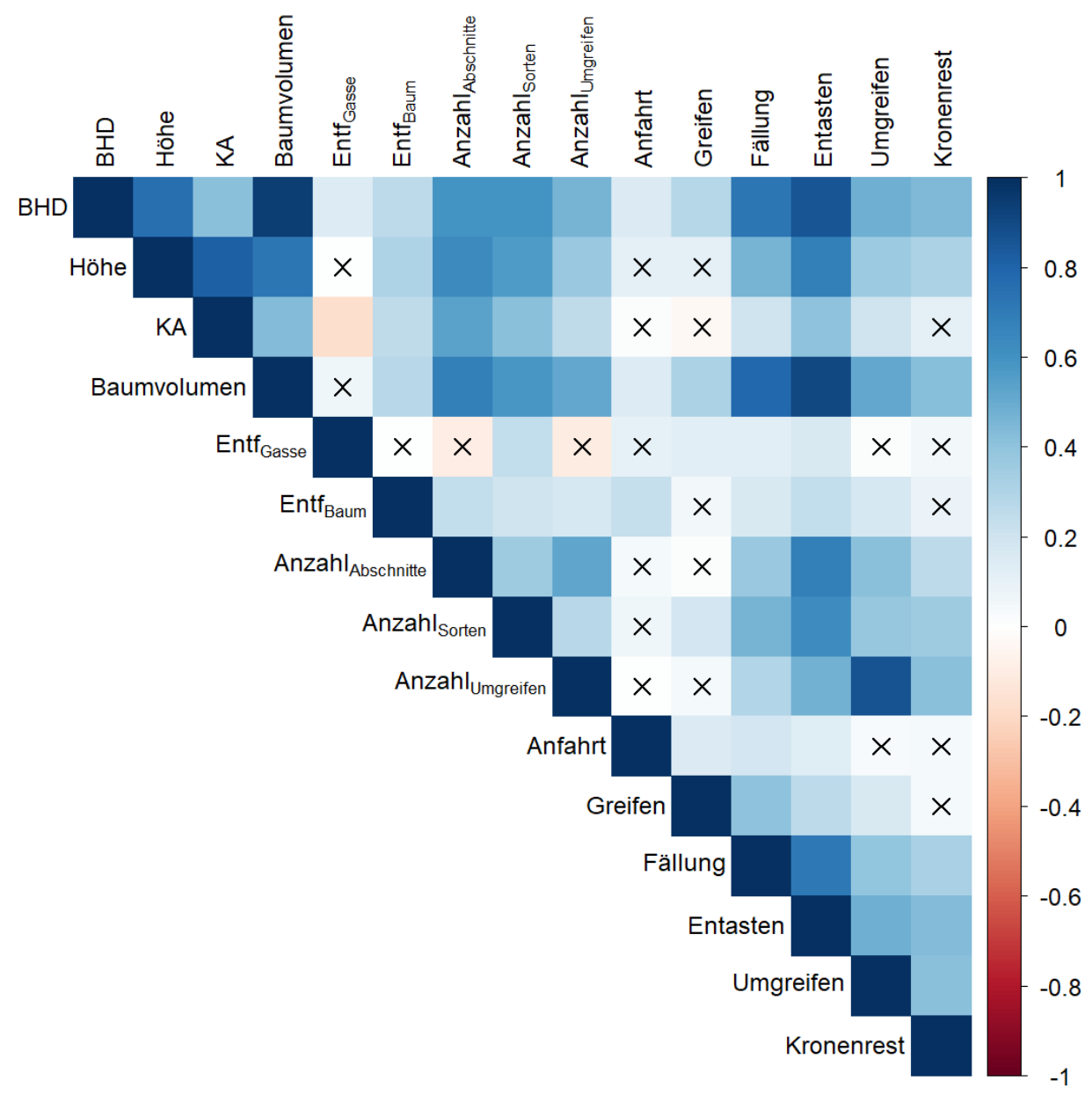

Abbildung 20: Korrelationsmatrix der metrischen Variablen auf Baumebene. Der Farbgradient gibt die Stärke und Richtung des Korrelationskoeffizienten $r$ an. Die Variablenkorrelationen, die mit einem X markiert sind, zeigen keinen signifikanten Zusammenhang (Pearson, $p<0,05$ ). BHD in $\mathrm{cm}$, Höhe und KA in $\mathrm{m}$, Baumvolumen in $\mathrm{m}^{3}$, Entfernungen in m und alle Aktivitäten in Sekunden; $N=254$.

Im nächsten Schritt wird der Zeitbedarf jeder Aktivität in einem separaten Modell im Detail analysiert. Jedes Modell wird zusätzlich auf Wechselwirkungen getestet. Um die Komplexität des Modells nicht unnötig zu erhöhen, werden nicht alle möglichen Wechselwirkungen getestet, sondern nur ausgewählte berücksichtigt. So wird beispielsweise 
Ergebnisse

nicht auf Wechselwirkungen zwischen den dimensionsbeschreibenden Variablen BHD und Baumvolumen getestet. Eine signifikante Wechselwirkung dieser beiden Variablen hätte die Interpretierbarkeit der Einzelvariablen in Bezug auf die abhängige Variable unnötig erschwert.

\subsubsection{Anfahrt und Positionierung}

Anfahrt und Positionierung wurden in bisherigen Studien als fixe Zeitanteile bzw. als volumenunabhängige Aktivitäten verstanden (Kapitel 4.4.2). Im Sinne des Stückvolumengesetzes (Hilf, 1928) erfolgt im Rahmen der vorliegenden Arbeit dennoch die Überprüfung des Zusammenhanges zwischen der Anfahrts- und Positionierungszeit und dem Baumvolumen (Abbildung 21).

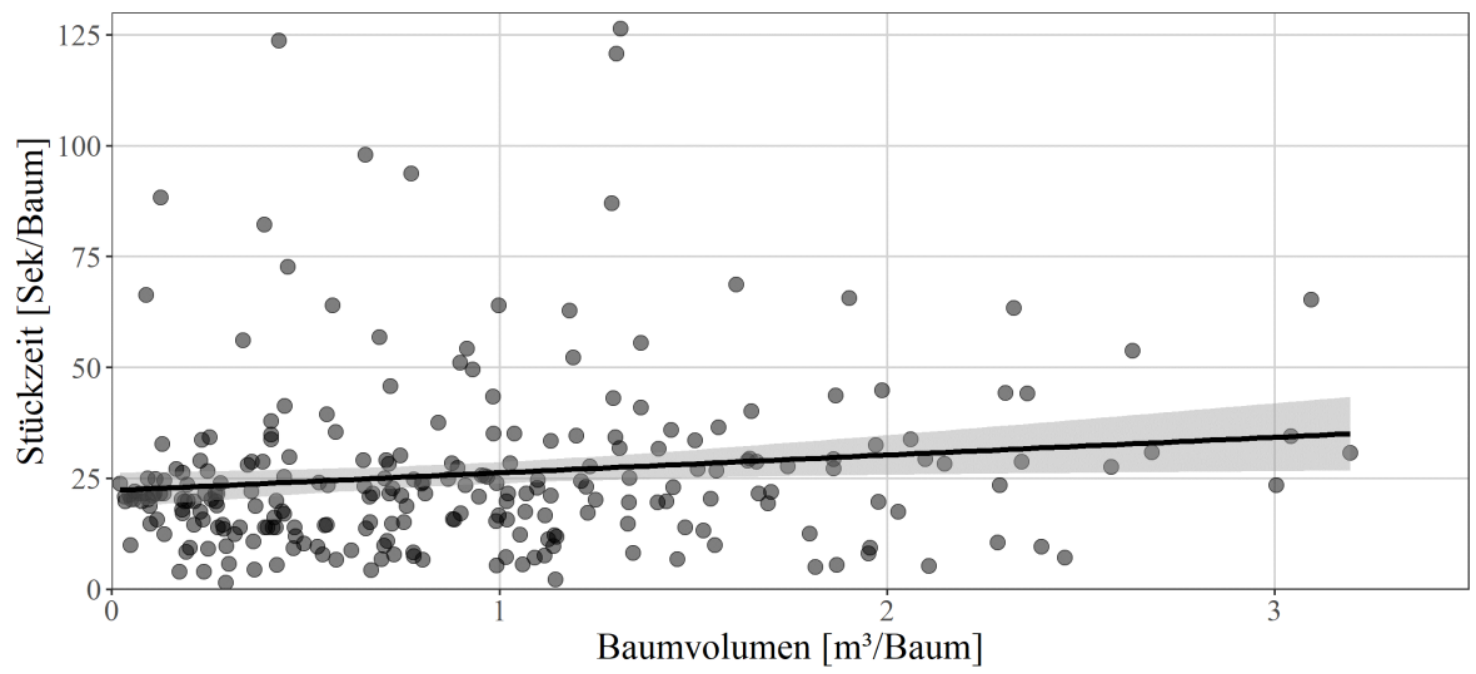

Abbildung 21: Abhängigkeit der Anfahrtszeit vom Baumvolumen in den Untersuchungsbeständen. Die graue Einfärbung zeigt den Vertrauensbereich der Funktion bei einem Konfidenzintervall $=0,95 ; N=254 ; R^{2}=0,02$.

Es wird ersichtlich, dass kein linearer (oder anderweitiger) Zusammenhang in den Daten vorliegt. Die Anfahrtszeit ist also tatsächlich unabhängig vom Baumvolumen. Ein wahrscheinlicherer variabler Kostentreiber besteht hingegen in der zum Baum zurückgelegten Entfernung bei der Anfahrt (Abbildung 22). Die Grafik zeigt einen positiv gerichteten Zusammenhang für die benötigte Zeit mit steigender Entfernung zum Entnahmebaum. 


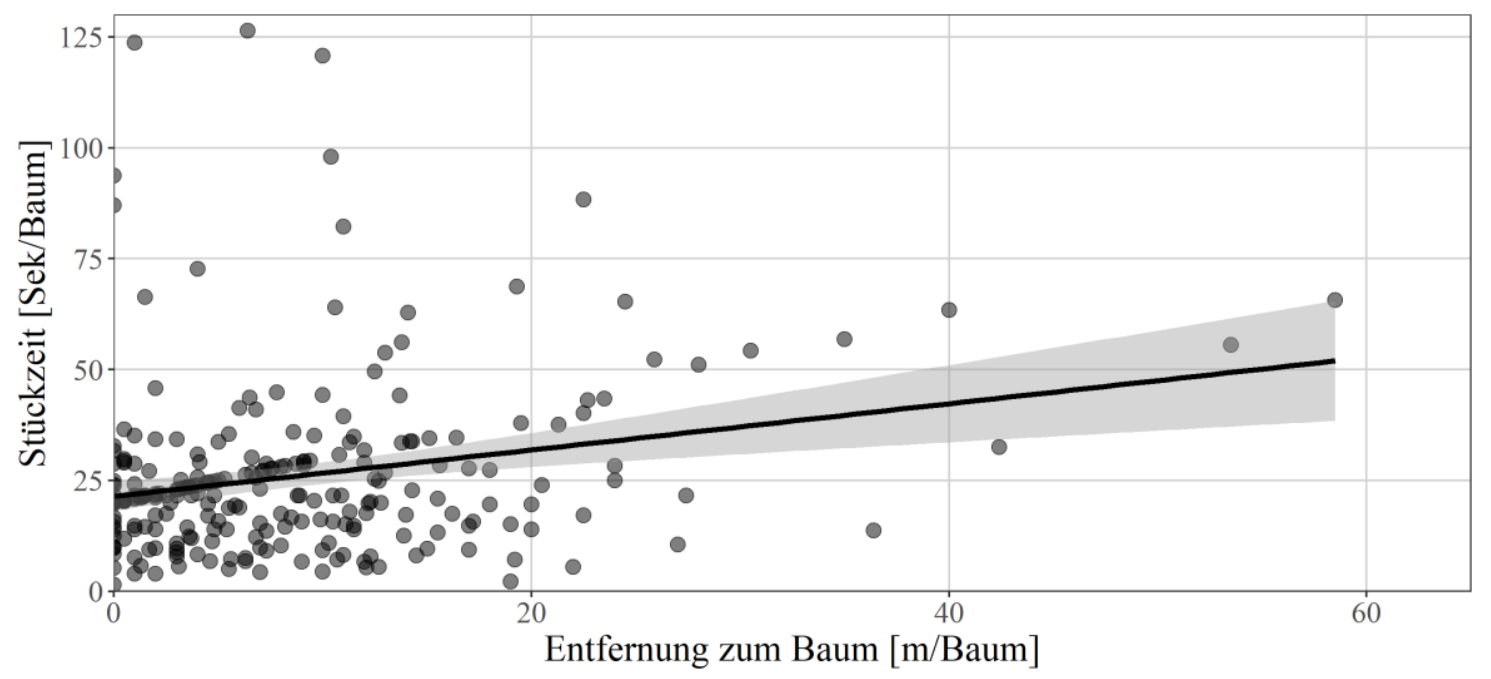

Abbildung 22: Abhängigkeit der Anfahrtszeit von der zurückgelegten Entfernung zu den Entnahmebäumen. Die graue Einfärbung zeigt den Vertrauensbereich der Funktion bei einem Konfidenzintervall $=0,95 ; N=254 ; R^{2}=0,05$.

Das Modell für die Anfahrt und Positionierung wird in Formel 8 und die statistische Regressionszusammenfassung in Tabelle 10 dargestellt. Dieses Modell zeigt nur die signifikanten Parameter nach vorgeschalteter Modellselektion (Kapitel 6.3.4). Die Aktivität benötigt eine fixe Zeit von rund 22 Sekunden je Entnahmebaum. Mit jedem zusätzlichen zurïckgelegten Meter Entfernung zum Baum, erhöht sich der Zeitbedarf um weitere 0,44 Sekunden. Eine Übersicht über die weiteren getesteten Variablen ist Anhang 3 zu entnehmen. Es konnte kein signifikanter Unterschied zwischen den Harvestersystemen festgestellt werden, was für einen ähnlichen Bewegungsablauf innerhalb der Fläche spricht.

$$
f(\text { Anfahrt })=\beta_{0}+\beta_{\text {Entf }} * \text { Entf }
$$

Formel 8: Lineare Regressionsfunktion des Zeitverbrauches (Sekunden/Baum) der Aktivität - Anfahrt und Positionierung. Legende siehe Variablenverzeichnis. $N=254$; $R^{2}=0,05$.

Tabelle 10: Regressionskoeffizient $b$, Standardfehler $S E$ und Signifikanzwert $p$ für die lineare Regression des Zeitverbrauchs (Sekunden/Baum) der Aktivität Anfahrt und Positionierung. Legende siehe Variablenverzeichnis. $N=254 ; A I C=2.223$.

\begin{tabular}{lccc}
\hline Faktor & $\mathbf{b}$ & $\mathbf{S E}$ & $\mathbf{p}$ \\
\hline$\beta_{0}$ & 22,02 & 1,58 & $<, 001 * * *$ \\
$\beta_{\text {Entf }}$ & 0,44 & 0,11 & $<, 001 * * *$ \\
\hline
\end{tabular}


Ergebnisse

Fälle, in denen die gefilmte Anfahrt und die vorher vermessene Fahrdistanz nicht übereinstimmten, wurden von dieser Analyse ausgeschlossen, da keine Aussagen über die tatsächlich zurückgelegten Distanzen abgeleitet werden konnten. $\mathrm{Zu}$ beachten ist, dass eine positiv gerichtete, nicht signifikante Wechselwirkung zwischen der zurückgelegten Entfernung zum Entnahmebaum und seinem Volumen besteht (Anhang 2, $p=0,21 ; n s$ ). Diese Wechselwirkung leuchtet ein, da Bäume mit einem höheren Volumen einen größeren Standraum beanspruchen und deshalb tendenziell weiter auseinander stehen. Konträr dazu wirkt sich der Strukturreichtum der Versuchsflächen aus (vgl. Einzelbaumvolumen in Tabelle 9). Dieses Ergebnis wird zusätzlich durch eine weitere Besonderheit dieser Aktivität beeinflusst - der gleichzeitigen Anfahrt von mehreren Entnahmebäumen. Das bedeutet, dass die Maschine von einer angefahrenen Position aus mehrere Entnahmebäume greifen und aufarbeiten kann. Dies wird durch den großen Aktionsradius der Maschinen und eine vorrauschauende Positionierung durch die Maschinenführer begünstigt. Die benötigte Anfahrtszeit wurde in der Auswertung gleichmäßig auf die betroffenen Bäume aufgeteilt und schwächt somit die Korrelation zwischen der Entfernung und dem Volumen der Entnahmebäume ab. Da das Volumen keinen signifikanten direkten (Abbildung 21) als auch keinen indirekten Einfluss über die Wechselwirkung mit der zurückgelegten Entfernung zum Baum besitzt, wurde die Variable in der AIC-Analyse nicht weiter berücksichtigt.

Wie bereits Speidel (1952, S. 29) für die manuelle Holzernte beschrieben hat (,Aufsuchen des Baumes“), ist die Anfahrt und Positionierung eine Aktivität mit einem hohen Fixanteil und einer geringen variablen Abhängigkeit. Dieser ist hauptsächlich von der zurückgelegten Entfernung abhängig, was die Befunde von Stampfer und Steinmüller (2004) bestätigen. Die Auswirkungen der Entfernung auf die Produktivität des Gesamtprozesses beleuchtet Kapitel 7.2.

\subsubsection{Greifen}

Die Tätigkeit des Greifens wurde in bisherigen Studien weitestgehend der Anfahrt und Positionierung (Purfürst, 2009, S. 53) oder der Fällung zugeordnet (von Bodelschwingh, 2006, S. 100). In dieser Untersuchung wurde diese Tätigkeit isoliert betrachtet, um sie genauer zu beschreiben. Aus diesem Grund wurde in der stehenden Aufnahme der Versuchsflächen die Distanz zwischen dem Entnahmebaum und der nächstliegenden Gassenmitte vermessen. Eine Besonderheit dieser Tätigkeit ist das Auftreten von zusätzlichen 
Aktivitäten bzw. einer indirekten Ausführung des Greifens (Kapitel 6.2). Indem sich der Kran- bzw. das Aggregat nicht auf direktem Weg zum Entnahmebaum bewegt, wird eine Verzögerung erzeugt. Die Einflussstärke der Zusatzaktivität auf die benötigte Zeit für das Greifen des Entnahmebaumes beträgt volumenunabhängig rund 10 Sekunden und ist in Abbildung 23 dargestellt.
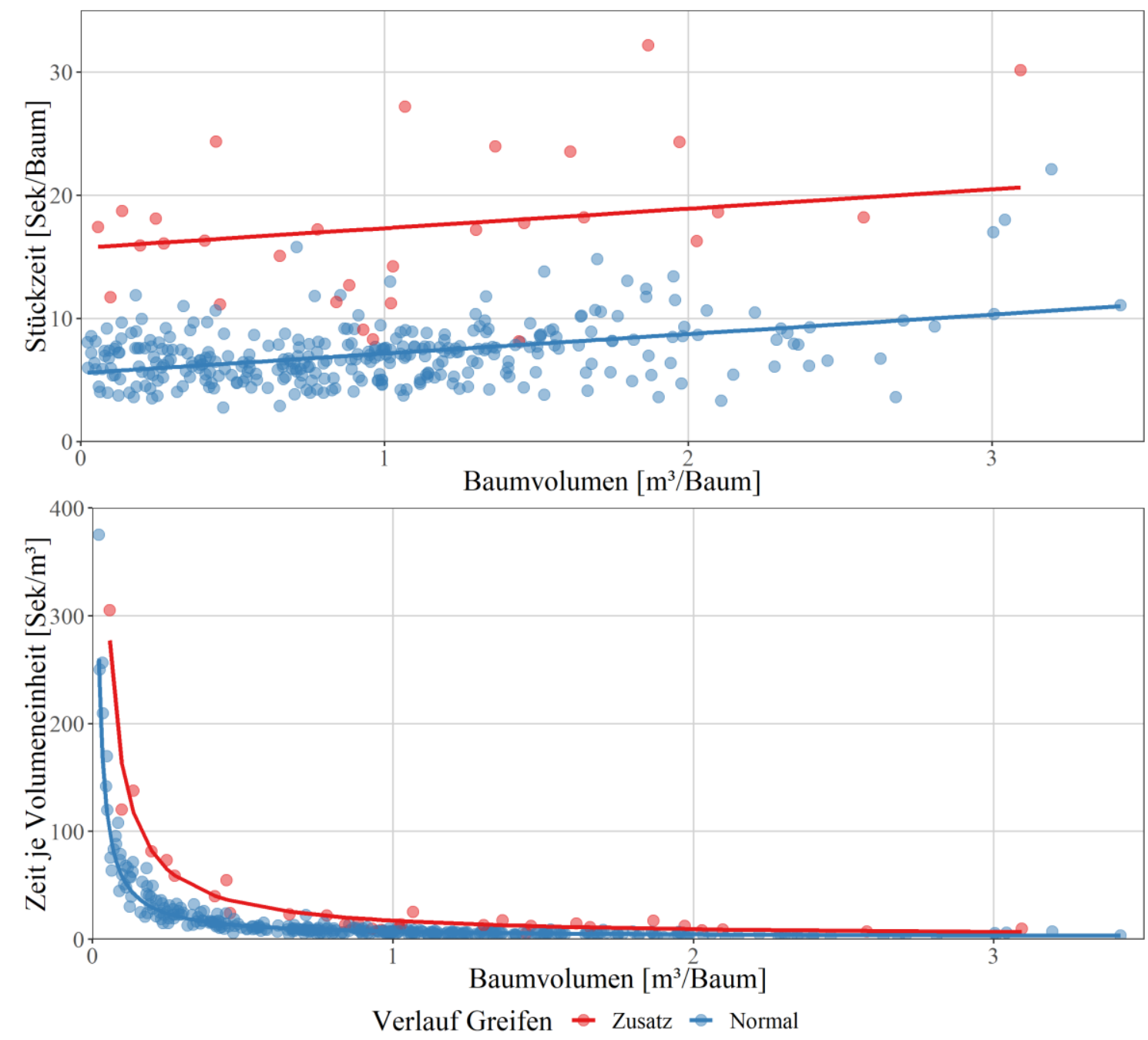

Abbildung 23: Oben: Stückzeit für die Aktivität Greifen in Abhängigkeit vom Baumvolumen. Unten: Zeit je $\mathrm{m}^{3}$ für die Aktivität Greifen in Abhängigkeit vom Baumvolumen. Die Verlaufsvariable beschreibt eventuelle Zusatzaktivitäten wie beispielsweise das Entfernen von Unterwuchs und wurde pro Baum Dummy-codiert. $N=361 ; R^{2}=0,57$.

Je nach Baumvolumen verdoppelt sich die benötigte Zeit, sobald das Greifen in einem indirekten Arbeitsablauf erfolgt. Zusätzlich ist der Einfluss des Baumvolumens auf die benötigte Zeit erkennbar - je volumenhaltiger der Baum ist, desto länger benötigt das Harvestersystem, diesen zu ergreifen. 
Speziell im hyperbelförmigen Verlauf (Kapitel 3.3.1) zeigt sich der Stückvolumenzusammenhang. Bei Bäumen mit einem geringen Volumen $\left(<0.25 \mathrm{~m}^{3}\right)$ wird ein Vielfaches der Zeit pro $\mathrm{m}^{3}$ benötigt als bei stärker dimensionierten Bäumen. Das lineare Gesamtmodell für die Aktivität Greifen ist in Formel 9 dargestellt und die statistische Regressionszusammenfassung in Tabelle 11.

$$
\begin{aligned}
f(\text { Greifen }) & =\beta_{0}+\beta_{V o l} * \text { Vol }+\beta_{\text {ZusatzG } 1} * \text { Zusatz }_{1} \\
& +\beta_{\text {System } 2} * \text { System }_{2}+\beta_{\text {System } 3} * \text { System }_{3}
\end{aligned}
$$

Formel 9: Lineare Regressionsfunktion des Zeitverbrauches (Sekunden/Baum) der Aktivität Greifen. Legende siehe Variablenverzeichnis. $N=361 ; R^{2}=0,62$.

Tabelle 11: Regressionskoeffizient $b$, Standardfehler $S E$ und Signifikanzwert $p$ für die lineare Regression des Zeitverbrauchs (Sekunden/Baum) der Aktivität Greifen. Legende siehe Variablenverzeichnis. $N=361 ;$ AIC $=1.711$.

\begin{tabular}{lccc}
\hline Faktor & b & SE & p \\
\hline$\beta_{0}$ & 5,27 & 0,27 & $<, 001^{* * *}$ \\
$\beta_{\text {Vol }}$ & 1,59 & 0,20 &, 093 \\
$\beta_{\text {ZusatzG } 1}$ & 9,93 & 0,49 & $<, 001^{* * *}$ \\
$\beta_{\text {System } 2}$ & $-1,04$ & 0,41 &, $011^{*}$ \\
$\beta_{\text {System } 3}$ & 1,31 & 0,29 & $<, 001^{* * *}$ \\
\hline
\end{tabular}

Das Greifen zeichnet sich durch einen starken fixen Zeitanteil je Baum aus. Dieser wird zusätzlich durch die Dummy-codierte Verlaufsvariable und das eingesetzte Harvestersystem verändert. Als variable Einflussgröße wirkt ausschließlich das Baumvolumen. Eine Übersicht über alle getesteten Variablen und Wechselwirkungen gibt Anhang 4. Darunter fallen auch Baumvariablen wie der Kronentyp und die Qualität, bei denen lediglich ein nicht signifikanter Einfluss nachgewiesen werden konnte und diese daher in der AICAnalyse aus dem Modell entfernt wurden. Entgegen der Erwartungen zeigt die Distanz zwischen der Rückegasse und dem Entnahmebaum keinen signifikanten Einfluss auf den Zeitverbrauch des Greifens. Ein Teil der Erklärung liegt in der Differenz des gemessenen und der tatsächlich zurückgelegten Entfernung zwischen dem Harvestersystem und dem Entnahmebaum während des Greifens. Aufgrund des vergleichsweise schwachen Volumentrends scheint das Greifen einen eher fixen Zeitverbrauch hinsichtlich des Gesamtprozesses einzunehmen. 


\subsubsection{Fällung}

Laut bisherigen Studien wird der für eine Fällung benötigte Zeitbedarf stark vom Baumvolumen beeinflusst (u. a. Nurminen et al., 2006, S. 344). Aus diesem Grund war es zu erwarten, dass dieses Modell dem klassischen linearen Stückvolumengesetz folgt (Abbildung 24).
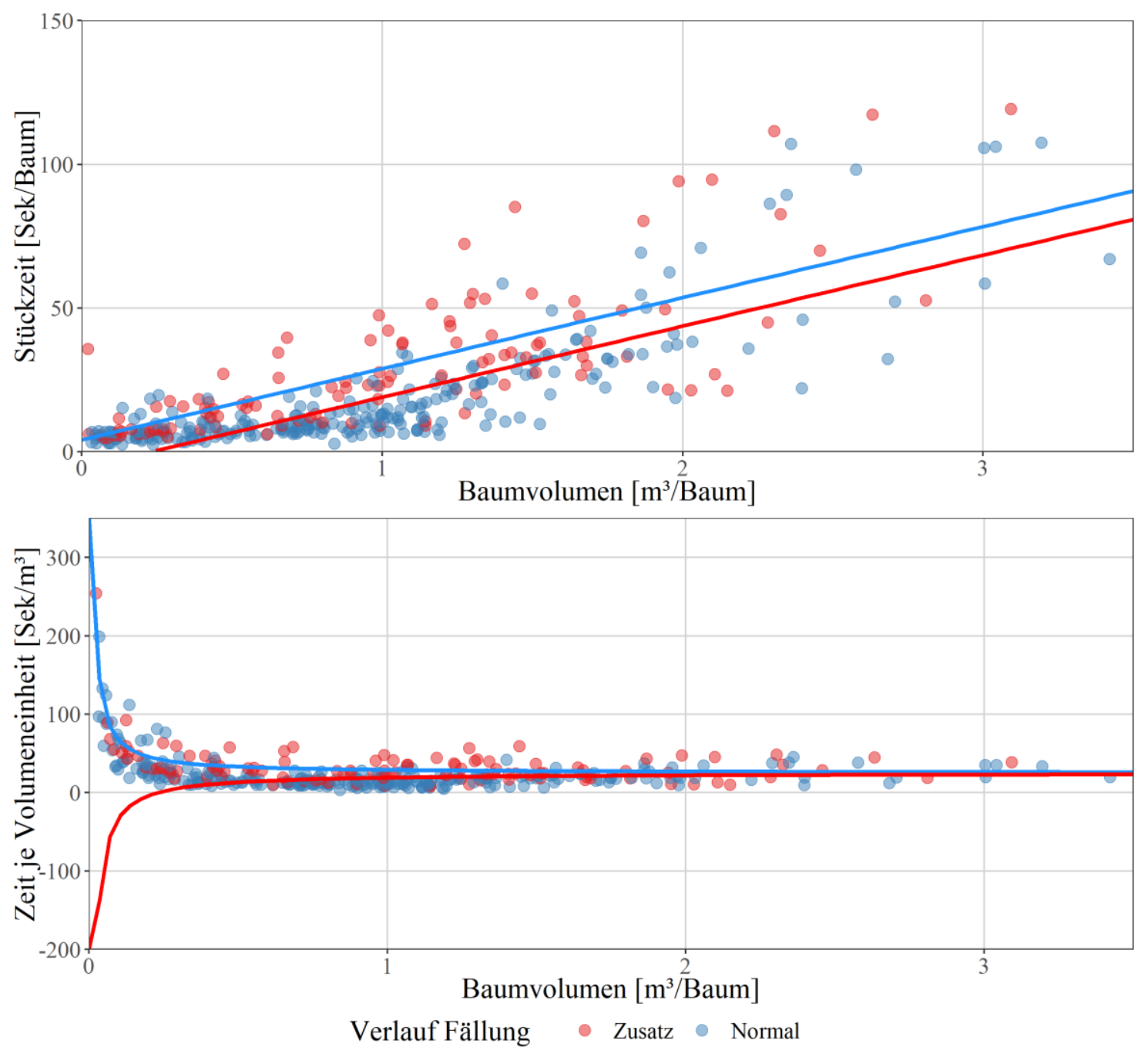

Abbildung 24: Oben: Stückzeit für die Aktivität Fällung in linearer Abhängigkeit vom Baumvolumen. Unten: Zeit je $\mathrm{m}^{3}$ für die Aktivität Fällung in Abhängigkeit vom Baumvolumen. Die Verlaufsvariable beschreibt eventuelle Zusatzaktivitäten wie beispielsweise ein Säuberungsschnitt und wurde pro Baum Dummy-codiert. $N=361 ; R^{2}=0,64$.

Die Abbildung verdeutlicht jedoch, dass die Aktivität nicht linear modellierbar ist. Obwohl das Bestimmtheitsmaß hoch ist, zeigt die visuelle Überprüfung des Modells inklusive der Umformulierung in die Hyperbelform zweifelsfrei, dass das lineare Modell nicht 
Ergebnisse

valide ist. Der Grund hierfür ist, dass es mit steigendem Baumvolumen zu einem überproportionalen Zeitbedarf für die Fällung kommt, welcher unabhängig von anfallenden Zusatzaktivitäten auftritt. Die graphische Darstellung verdeutlicht vielmehr, dass der Zusammenhang zwischen der Stückzeit und dem Baumvolumen überproportional ist. Wie aus dem theoretischen Kapitel 3.3.2 hervorgeht, ist die Modellierung eines quadratischen Zusammenhanges zwischen Baumvolumen und Stückzeit möglich und auch durchaus mit der Theorie des Stückvolumengesetzes vereinbar. Die Zeit je Volumeneinheit würde in einem solchen quadratischen Modell nach einem starken (hyperbelförmigen) Abfall wieder ansteigen.
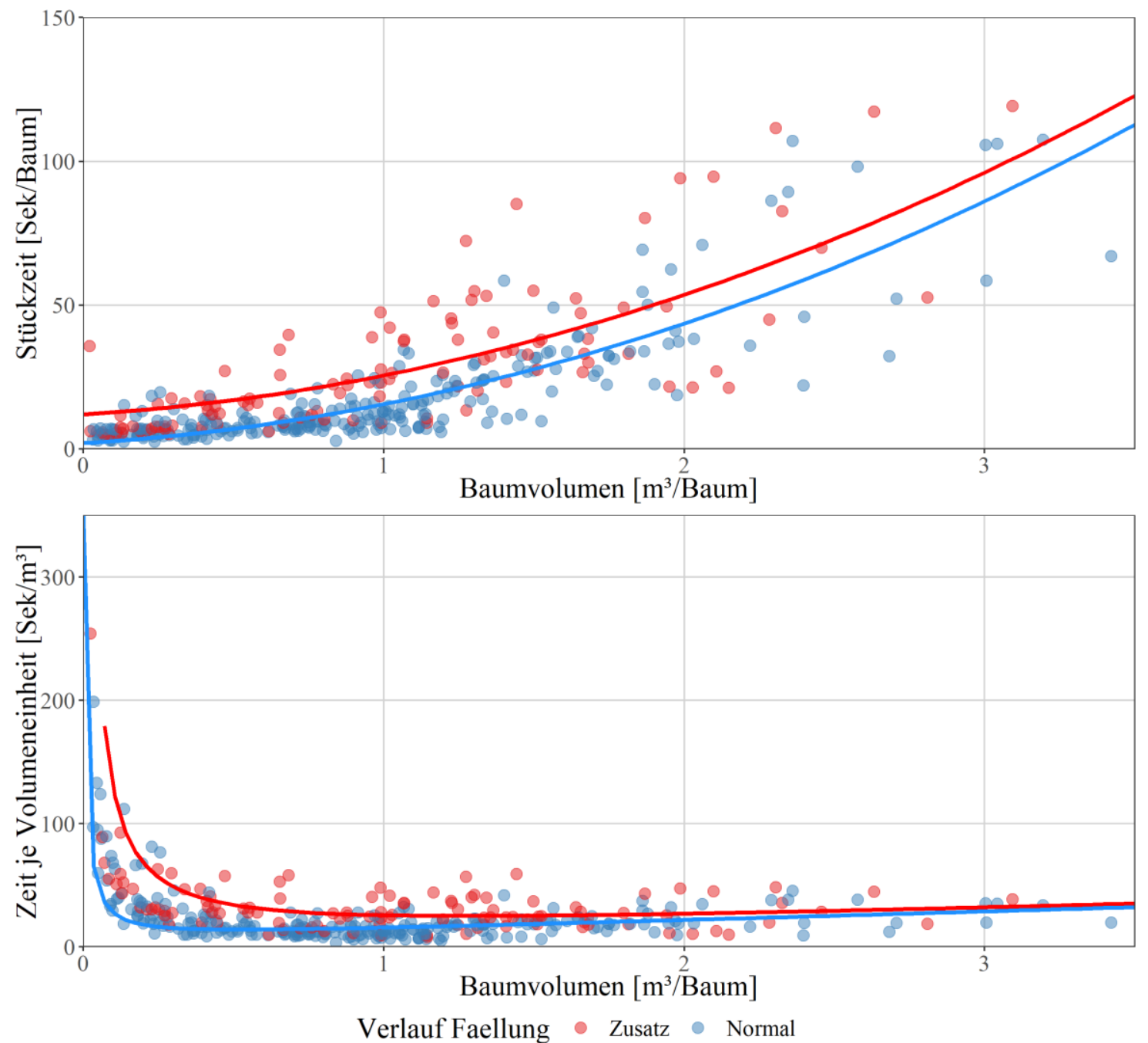

Abbildung 25: Oben: Stückzeit für die Aktivität Fällung in quadratischer Abhängigkeit vom Baumvolumen. Unten: Zeit je $\mathrm{m}^{3}$ für die Aktivität Fällung in Abhängigkeit vom Baumvolumen. Die Verlaufsvariable beschreibt eventuelle Zusatzaktivitäten wie beispielsweise ein Säuberungsschnitt und wurde pro Baum Dummy-codiert; $N=361 ; R^{2}=$ 0,77 . 
Abbildung 25 zeigt ohne jeden Zweifel, dass ebendieser Zusammenhang dem Zeitbedarfsmodell der Fällung zugrunde liegt. Im Gegensatz zu dem linearen Ausgangsmodell (Abbildung 24) eignet sich der quadratische Ansatz augenscheinlich sehr gut zur Beschreibung des Zusammenhanges zwischen dem Zeitbedarf der Fällung und dem Baumvolumen. Auch im unteren Volumenbereich erfolgt nun eine realistischere Schätzung der benötigten Zeit. Zusätzlich zum Volumen konnten noch weitere Variablen in das Modell integriert werden (Formel 10, Tabelle 12).

$$
\begin{aligned}
f(F \text { ällung }) & =\beta_{0}+\beta_{V o l 1} * V o l+\beta_{V o l 2} * V_{o l}{ }^{2}+\beta_{S 1} * S 1+\beta_{Z 1} * Z 1+\beta_{L 1} * L 1 \\
& +\beta_{\text {Qualität }} * \text { Qualität }+\beta_{\text {System } 2} * \text { System }_{2}+\beta_{\text {System } 3} * \text { System }_{3}
\end{aligned}
$$

Formel 10: Regressionsfunktion des Zeitverbrauchs (Sekunden/Baum) der Aktivität Fällung. Legende siehe Variablenverzeichnis. $N=361 . R^{2}=0,77$.

Tabelle 12: Regressionskoeffizient $b$, Standardfehler $S E$ und Signifikanzwert $p$ für die quadratische Regression des Zeitverbrauchs (Sekunden/Baum) der Aktivität Fällung. Legende siehe Variablenverzeichnis. $N=361 ;$ AIC $=2.738$.

\begin{tabular}{lccc}
\hline Faktor & $\mathbf{b}$ & $\mathbf{S E}$ & $\mathbf{p}$ \\
\hline$\beta_{0}$ & $-5,46$ & 3,06 &, 075 \\
$\beta_{\text {Vol } 1}$ & 6,70 & 2,62 &, $011^{*}$ \\
$\beta_{\text {Vol2 }}$ & 6,55 & 0,94 & $<, 001^{* * *}$ \\
$\beta_{S 1}$ & 4,41 & 2,08 &, $035^{*}$ \\
$\beta_{Z 1}$ & 7,50 & 1,46 & $<, 001^{* * *}$ \\
$\beta_{L 1}$ & 12,22 & 2,44 & $<, 001^{* * *}$ \\
$\beta_{\text {Qualität }}$ & 1,22 & 0,60 &, $042^{*}$ \\
$\beta_{\text {System } 2}$ & $-3,23$ & 1,73 &, 063 \\
$\beta_{\text {System } 3}$ & 8,70 & 1,27 & $<, 001^{* * *}$ \\
\hline
\end{tabular}

An diesem Punkt kann ein erstes Zwischenfazit für die zweite und dritte Forschungshypothese gezogen werden. Durch die Transformation der abhängigen Variablen wird eine negative Grenzproduktivität modelliert (H2). Für die Aktivität Fällung kann dieser Punkt bei einem Baumvolumen von rund 1,4 $\mathrm{m}^{3}$ lokalisiert werden. Des Weiteren zeigt sich ein signifikanter Effekt der Stammqualität auf den Zeitverbrauch der Fällung (H3). Dieser fällt aber im Vergleich zu den anderen Variablen gering aus.

Neben dem Volumen und der Qualität haben auch andere Kostentreiber einen signifikanten Einfluss auf die benötigte Fällzeit. Eine Besonderheit sind die jeweiligen Gründe für die zusätzliche Aktivität während der Fällung - der Säuberungsschnitt (S1), ein Ziehen 
Ergebnisse

des Stammes (Z1) sowie ein eventuelles Lösen des Aggregates bei der Fällung in Verbindung mit einem erneuten Greifen, um die Fällung abzuschließen (L1). Es wird ersichtlich, dass jede dieser Zusatzaktivitäten eine zusätzliche Zeit in unterschiedlicher Höhe in Anspruch nimmt. Der größte Mehraufwand wird verursacht, wenn der Maschinenführer den Stamm bei der Fällung loslassen muss. Weitere Analysen zeigen zudem, dass die Eintrittswahrscheinlichkeiten dieser Zusatzaktivität mit dem Baumvolumen ansteigen (Anhang 5).

Eine Übersicht über alle getesteten Variablen gibt Anhang 6. Ein Beispiel für die eliminierten Variablen sind die Dummy-codierte Verlaufsvariable, die angibt, ob überhaupt ein Zusatzaktivität durchgeführt wurde. Es wird also anhand des Modellergebnisses offensichtlich, dass die Gründe für Zusatzzeiten so vielfältig und relevant sind, dass deren separate Modellierung eine bessere Modellerklärung liefert als eine gemeinsame, vereinheitlichte Betrachtung.

\subsubsection{Entasten}

Nach der Anfahrt und Positionierung nimmt das Entasten des Baumes den zweitgrößten Zeitanteil am Gesamtprozess ein (Abbildung 19). In der bestehenden Theorie wird das Entasten als Kernaktivität der Aufarbeitung verstanden, welche im erheblichen Maße vom Volumen des Entnahmebaumes beeinflusst wird (Nurminen et al., 2006, S. 357). Das Entasten kann auf zwei unterschiedlichen Ebenen analysiert werden: auf Baum- und Abschnittsebene. In einem ersten Schritt wird die Aktivität auf Abschnittsebene genauer beleuchtet. Abbildung 26 zeigt einen deutlichen linearen Zusammenhang der Stückzeit über dem Stückvolumen. Auch wird sichtbar, dass die Verlaufsvariable (Normal/Zusatz) einen großen Teil der Streuung erklärt und die Daten sinnvoll in zwei Gruppen trennt, von denen jede einzelne einen linearen Verlauf zeigt. 


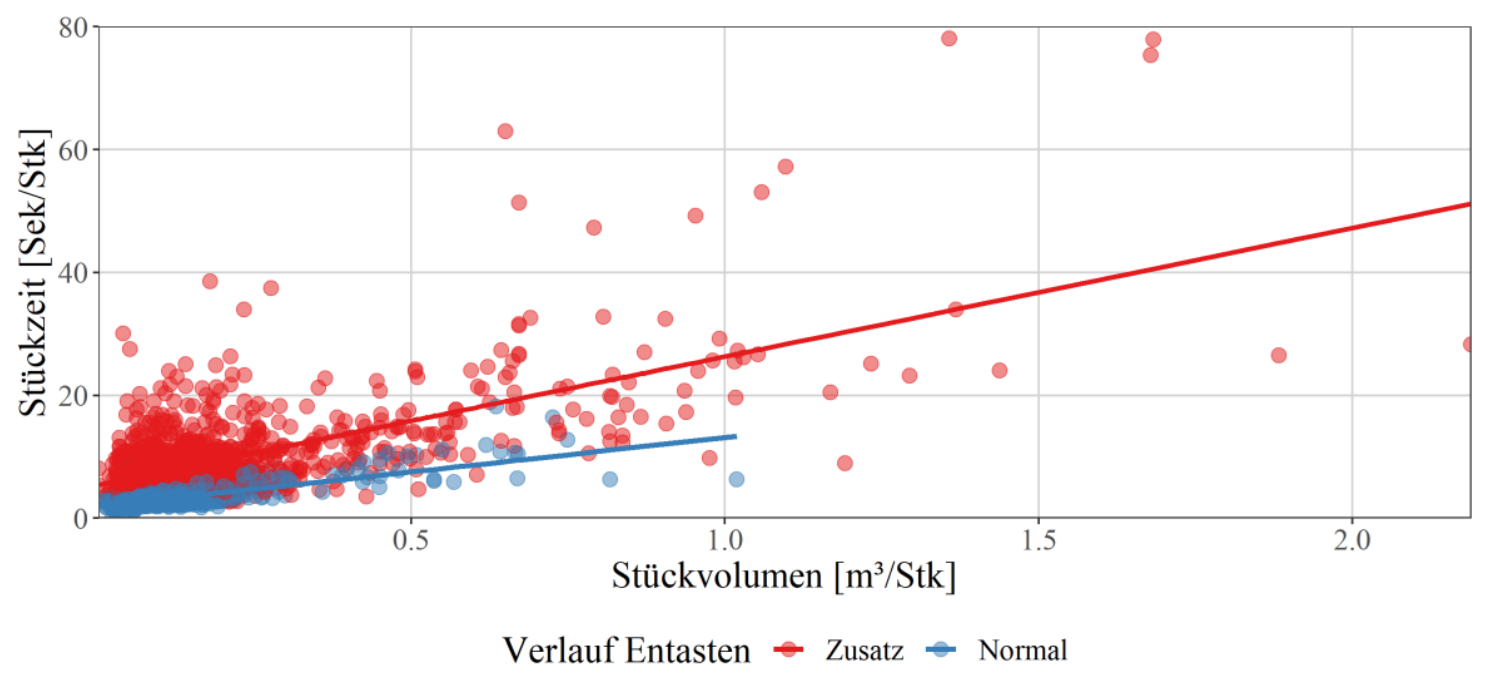

Abbildung 26: Stückzeit für die Aktivität Entastung in linearer Abhängigkeit vom Volumen der Abschnitte. Die Verlaufsvariable beschreibt eventuelle Zusatzaktivitäten während der Aktivität, wie beispielsweise das Ziehen des Abschnittes. $N=2300 ; R^{2}=0.59$.

Interessant ist, dass bei Abschnitten ab etwa einem $\mathrm{m}^{3}$ stets Zusatzaktivitäten stattfanden. Der Hauptgrund liegt hierfür in einer notwendigen Ziehbewegung, um den Abschnitt zu manipulieren. Formel 11 zeigt die Funktionsgleichung des Entastungsmodells auf Abschnittsebene. Die statistischen Kennzahlen zur Regression sind in Tabelle 13 dargestellt.

$$
\begin{aligned}
f\left(\text { Entasten }_{\text {Abschnitt }}\right) & =\beta_{\text {Vol.Abs }} * \operatorname{Vol}_{A b s}+\beta_{I S} * I S+\beta_{P A} * P A+\beta_{S} * S \\
& +\beta_{S H} * S H+\beta_{V o l . A b s \times P A} * V_{\text {ol }} \times P A \\
& +\beta_{\text {Vol.Abs } \times S} * \text { Vol }_{A b s} \times S+\beta_{V o l . A b s \times S H} * \text { Vol }_{A b s} \times S H \\
& +\beta_{\text {ZusatzE } 0} * \text { Zusatz }_{0}+\beta_{\text {Herkunft } 1} * \text { Herkunft }_{1} \\
& +\beta_{A 1} * A 1+\beta_{M 1} * M 1+\beta_{S 1} * S 1+\beta_{Z 1} * Z 1 \\
& +\beta_{\text {System } 2} * \text { System }_{2}+\beta_{\text {System } 3} * \text { System }_{3}
\end{aligned}
$$

Formel 11: Lineare Funktion zur Erklärung der Entastungszeit auf Abschnittsebene (Sekunden/Abschnitt). Legende siehe Variablenverzeichnis. $N=2300 ; R^{2}=0,83$. 
Ergebnisse

Tabelle 13: Regressionskoeffizient $b$, Standardfehler $S E$ und Signifikanzwert $p$ für die lineare Regression des Zeitverbrauchs (Sekunden/Abschnitt) der Aktivität Entasten auf Abschnittsebene. Legende siehe Variablenverzeichnis. $N=2.300 ; A I C=12.586$.

\begin{tabular}{lccc}
\hline Faktor & $\mathbf{b}$ & $\mathbf{S E}$ & $\mathbf{p}$ \\
\hline$\beta_{\text {Vol.Abs }}$ & 13,87 & 1,20 & $<, 001^{* * *}$ \\
$\beta_{I S}$ & 5,73 & 0,29 & $<, 001^{* * *}$ \\
$\beta_{P A}$ & 4,58 & 0,75 & $<, 001^{* * *}$ \\
$\beta_{S}$ & 7,04 & 2,54 &, $005^{* *}$ \\
$\beta_{\text {SH }}$ & 3,75 & 1,00 & $<, 001^{* * *}$ \\
$\beta_{\text {Vol.Abs } \times \text { PA }}$ & 8,47 & 3,55 &, $017^{*}$ \\
$\beta_{\text {Vol.Abs } \times S}$ & $-0,85$ & 4,89 &, 861 \\
$\beta_{\text {Vol.Abs } \times \text { SH }}$ & 9,52 & 1,66 & $<, 001^{* * *}$ \\
$\beta_{\text {ZusatzE } 0}$ & $-3,47$ & 0,25 & $<, 001^{* * *}$ \\
$\beta_{\text {Herkunft } 1}$ & $-0,92$ & 0,18 & $<, 001^{* * *}$ \\
$\beta_{\text {A1 }}$ & 2,14 & 0,26 & $<, 001^{* * *}$ \\
$\beta_{\text {M1 }}$ & 2,53 & 0,40 & $<, 001^{* * *}$ \\
$\beta_{\text {S1 }}$ & 1,86 & 1,14 &, 102 \\
$\beta_{Z 1}$ & 0,56 & 0,26 &, $029 *$ \\
$\beta_{\text {System } 2}$ & $-0,93$ & 0,21 & $<, 001^{* * *}$ \\
$\beta_{\text {System } 3}$ & 0,06 & 0,20 &, 780 \\
\hline
\end{tabular}

Im Unterschied zur Fällung hat bei der Entastung der Abschnitte nicht nur die Verlaufsvariable an sich $(\mathrm{J} / \mathrm{N})$, sondern auch die jeweilige Ursache einen signifikanten Einfluss auf die benötigte Zeit. Ausgenommen der Ziehbewegung verursachen alle anderen $\mathrm{Zu}-$ satzaktivitäten einen ähnlichen Mehraufwand von rund zwei Sekunden. Stammabschnitte benötigen weniger Zeit als Abschnitte, die innerhalb des Kronenbereiches aufgearbeitet werden. Komplexer ist der Unterschied zwischen den Sortimenten. Ein Teil der Differenz kann durch die Wechselwirkung mit dem Volumen der Abschnitte erklärt werden. Mit steigendem Abschnittsvolumen steigt der Zeitbedarf der Paletten- und Stammholzabschnitte sehr viel stärker an als der Zeitbedarf der Industrie- und Schälholzabschnitte. Darüber hinaus unterscheiden sich die Sortimente innerhalb ihrer Entastungsdauer auch unabhängig des jeweiligen Volumens voneinander. Höherwertige Sortimente (PA, SH) weisen einen geringeren Grundzeitbedarf auf, ihr Zeitbedarf steigt aber viel stärker mit dem Volumen an.

Beim Wechsel der Betrachtungsebene auf das Objekt Baum müssen einige Abschnittvariablen transformiert werden. Dazu gehören neben den Verlaufsvariablen auch die Aushaltung der Abschnitte, die auf Baumebene nur als Anzahl an Sortimenten je Baum weiter 
genutzt werden kann, da auf Baumebene in der Regel nicht nur ein Sortiment ausgehalten wird. Andere Variablen können nicht weiter genutzt werden, da sie fest mit einem Abschnitt verknüpft sind (z. B. Herkunft des Sortiments aus dem Stamm- oder Kronenbereich). Darüber hinaus können aber auch baumspezifische Variablen (z. B. Qualität) in der Regression genutzt werden. Abbildung 27 zeigt den Zeitbedarf pro Baum für das Entasten. Ähnlich wie bei der Fällung erfordert das Entasten mit ansteigendem Baumvolumen einen überproportionalen Zeitbedarf, was in einen quadratischen Funktionsverlauf resultiert und in einer negative Grenzproduktivität mündet (Abbildung 27, unten). Wie bereits bei der Fällung, ist dies eine Bestätigung der Forschungshypothese H2. Zusätzlich ist die Anzahl der ausgehaltenen Sortimente als beeinflussende Variable signifikant. Je mehr Sorten pro Baum ausgehalten werden, desto mehr Zeit wird für das Entasten des Baumes benötigt. Dieser Zusammenhang bestätigt die Forschungshypothese H4.

Die Funktion je Variablenausprägung ist nur für den im Datensatz vorhandenen Gültigkeitsbereich dargestellt. Die in Formel 12 dargestellte Funktion zeigt das Gesamtmodell für die Aktivität Entasten auf Baumebene mit den statistischen Koeffizienten in Tabelle 14. 
Ergebnisse
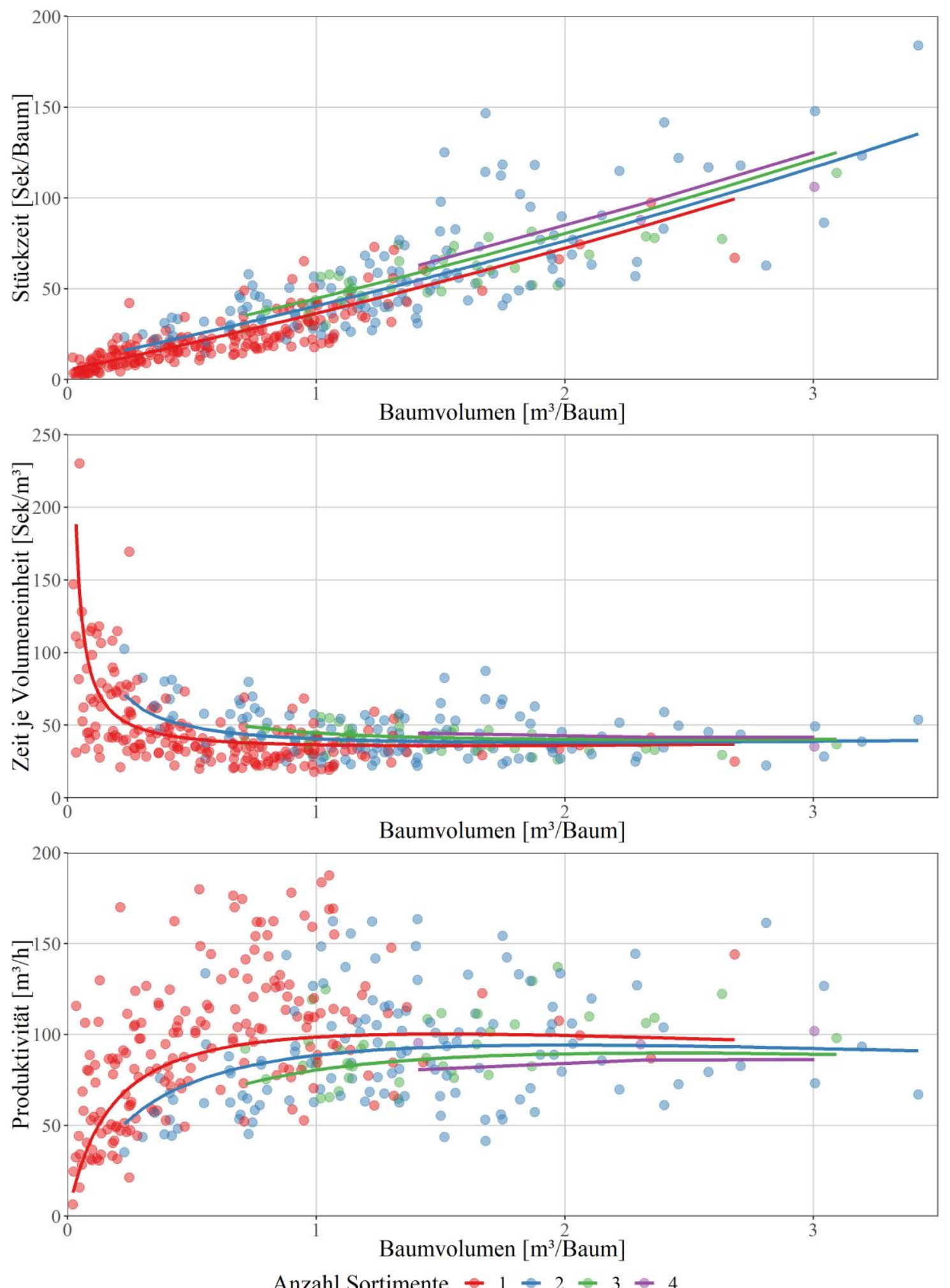

Abbildung 27: Oben: Stückzeit für die Aktivität Entasten in quadratischer Abhängigkeit vom Baumvolumen. Mitte: Zeit je $\mathrm{m}^{3}$ für die Aktivität Entasten in Abhängigkeit vom Baumvolumen. Unten: Produktivität für die Aktivität Entasten in Abhängigkeit vom Baumvolumen. Die Zusatzvariable beschreibt den Einfluss der Sortenanzahl auf die abhängige Variable. $N=361 ; R^{2}=0,78$. 


$$
\begin{aligned}
f(\text { Aufarbeitung }) & =\beta_{0}+\beta_{\text {Vol1 }} * \text { Vol }+\beta_{\text {Vol2 } 2} * \text { Vol }^{2} \\
& +\beta_{\text {Abschnitte }} * \text { Abschnitte }+\beta_{\text {Sorten }} * \text { Sorten } \\
& +\beta_{\text {Qualität }} * \text { Qualität }
\end{aligned}
$$

Formel 12: Quadratische Funktion zur Erklärung der Entastungszeit auf Baumebene (Sekunden/Baum). Legende siehe Variablenverzeichnis. $N=361 ; R^{2}=0,78$.

Tabelle 14: Regressionskoeffizient $b$, Standardfehler SE und Signifikanzwert $p$ für die quadratische Regression des Zeitverbrauchs (Sekunden/Baum) der Aktivität Entasten auf Baumebene. Legende siehe Variablenverzeichnis. $N=361 ;$ AIC $=2.928$.

\begin{tabular}{lccc}
\hline Faktor & $\mathbf{b}$ & $\mathbf{S E}$ & $\mathbf{p}$ \\
\hline$\beta_{0}$ & $-12,78$ & 4,24 &, $003^{* *}$ \\
$\beta_{\text {Vol1 }}$ & 21,75 & 4,02 & $<, 001^{* * *}$ \\
$\beta_{\text {Vol2 }}$ & 3,24 & 1,26 &, $01^{*}$ \\
$\beta_{\text {Abschnitte }}$ & 1,56 & 0,35 & $<, 001^{* * *}$ \\
$\beta_{\text {Sorten }}$ & 5,26 & 1,29 & $<, 001^{* * *}$ \\
$\beta_{\text {Qualität }}$ & 2,08 & 0,78 &, $008^{* *}$ \\
\hline
\end{tabular}

Neben dem Baumvolumen und der Anzahl ausgehaltener Sortimente pro Baum weisen die Qualität (H3) und die Anzahl an Abschnitten pro Baum einen signifikanten Einfluss auf den Zeitverbrauch des Entastens auf. Eine Übersicht über alle getesteten und eliminierten Variablen ist in Anhang 7 dargestellt. Überraschend ist, dass das Harvestersystem keinen signifikanten Einfluss auf die Entastungszeit auf Baumebene besitzt, obwohl dieser Faktor auf Abschnittsebene im Modell signifikant war.

Wie bereits angesprochen, können durch die Aggregation der Abschnittsvariablen auf die Baumebene einige Informationen nicht mehr genutzt werden. Zusätzlich werden aber weitere Variablen zugänglich. Dieser Zusammenhang wird am Beispiel der Sortimentsvielfalt nachfolgend genauer beleuchtet (Abbildung 28). 

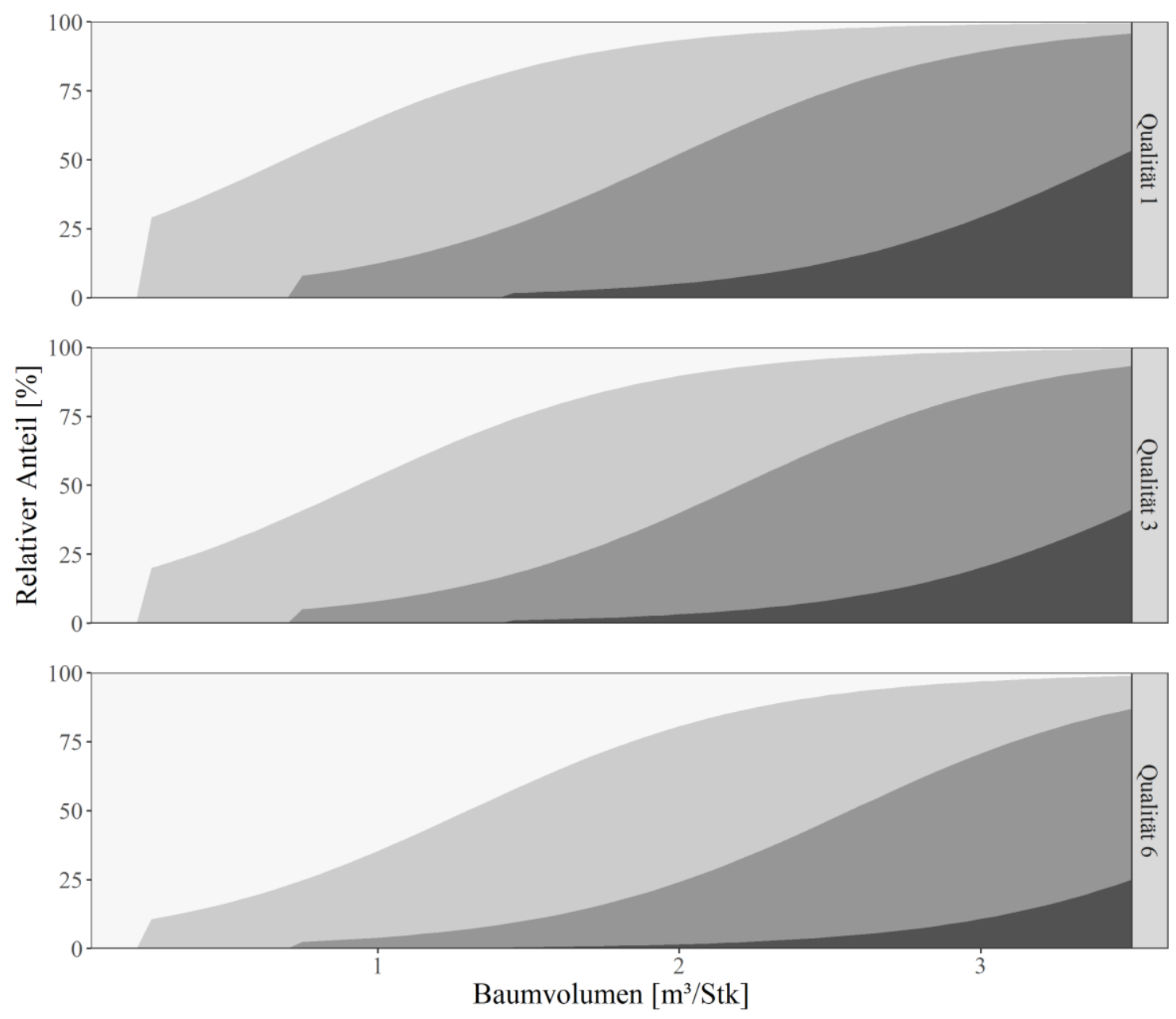

Anzahl Sorten $1 \square 2 \square 3 \square 4$

Abbildung 28: Modellierte relative Anteile der Sortenanzahl in Abhängigkeit zum Baumvolumen und der Qualität des Baumes. Dargestellt für die Qualitäten 1,3 und 6. $N=361$.

Abgebildet sind der Einfluss der Qualität und des Baumvolumens auf die Wahrscheinlichkeit der Anzahl ausgehaltener Sortimente pro Baum. Mit steigendem Baumvolumen steigt auch die Wahrscheinlichkeit, dass ein oder mehrere zusätzliche Sortimente ausgehalten werden. Dieser Zusammenhang unterscheidet sich zwischen Bäumen unterschiedlicher Qualitätsstufen. Mit sinkender Qualität verringern sich die relativen Wahrscheinlichkeiten weiterer Sortimente. Bei dieser Art der Aggregation geht allerdings die Information verloren, welches Sortiment ausgehalten wird. Wie im Anhang 8 und Anhang 9 sichtbar wird, existiert durchaus ein Unterschied in den relativen Wahrscheinlichkeiten der Sortimente, ob neben Industrieholz Palette oder Stammholz als zweites Sortiment ausgehalten wird. Da das Analysemodell durch diese feingliedrige Gruppierung jedoch sehr unübersichtlich werden würde, wird dieser Pfad nicht weiterverfolgt. 


\subsubsection{Umgreifen}

Während sich alle anderen auftretenden Aktivitäten entlang der mechanisierten Aufarbeitung auch in anderen Untersuchungen (Kapitel 6.2) wiederfinden, scheint das Umgreifen eine Besonderheit bei der Aufarbeitung von Buche zu sein. Auch analytisch gesehen unterscheidet sich die Modellerstellung der benötigten Zeit für das Umgreifen von den anderen Aktivitäten. Aus diesem Grund wird diese Aktivität in der vorliegenden Arbeit in drei Schritten näher betrachtet. Über ein logistisches Modell wird zunächst analysiert, von welchen Baumparametern die Wahrscheinlichkeit des Umgreifens abhängt. Im zweiten Schritt erfolgt eine geordnete logistische Regression, welche die Wahrscheinlichkeit der Anzahl der Umgriffe bei diesen Bäumen als abhängige Variable untersucht. Die zweite Regression wird unabhängig von der ersten durchgeführt. Den Abschluss bildet die eigentliche Zeitbedarfsbetrachtung dieser Aktivität im Rahmen eines linearen Modells.

In den bestehenden Studien (u. a. Guglhör, 1994, S. 697; Schorr, 2000, S. 522) wird hauptsächlich auf die Starkastigkeit bzw. den Kronentyp als Ursache für ein Umgreifen verwiesen. Dies konnte in der vorliegenden Arbeit bestätigt werden: So geht aus der logistischen Regression (Abbildung 29) ebenfalls hervor, dass der Kronentyp einen erheblichen Einfluss auf die Wahrscheinlichkeit eines Umgreifens besitzt.

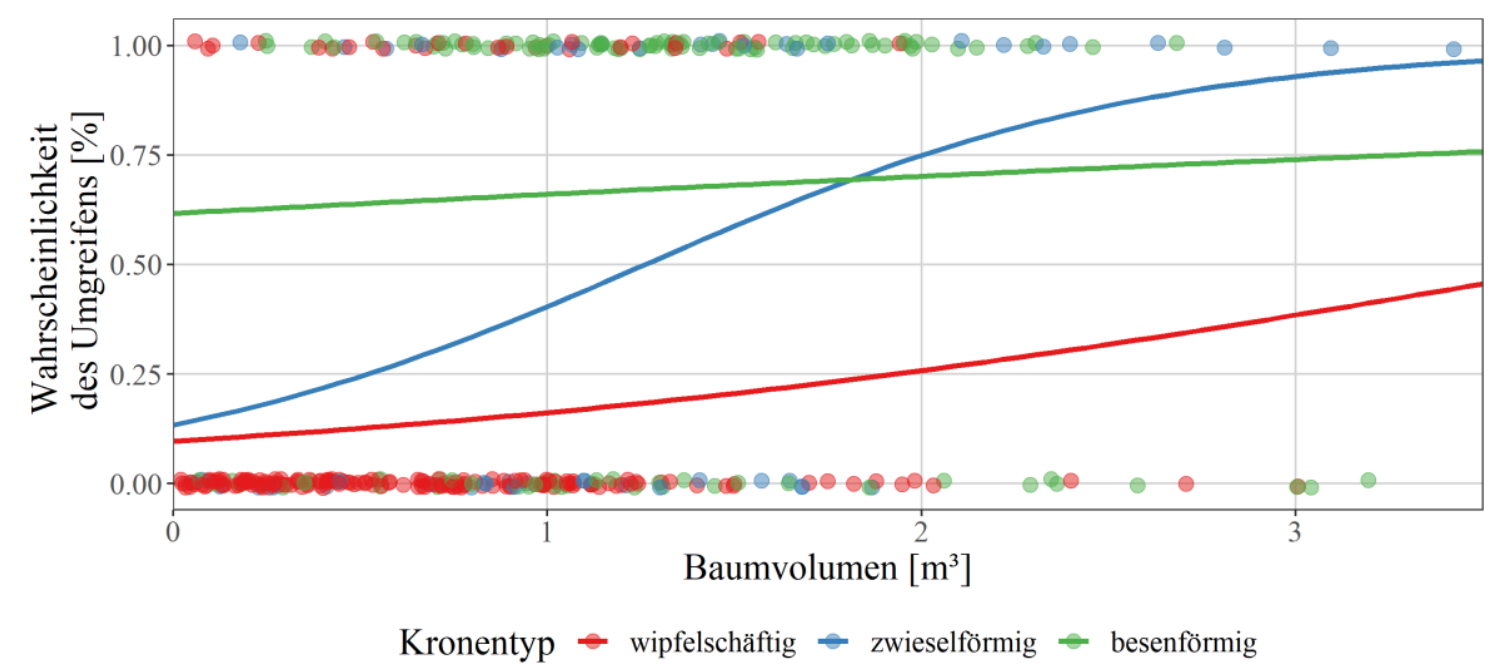

Abbildung 29: Wahrscheinlichkeit des Umgreifens in Abhängigkeit des Baumvolumens und Kronentyps. $N=361$. 
Ergebnisse

Bei wipfelschäftigen Buchen steigt die Wahrscheinlichkeit - selbst bei einem Baumvolumen über 2,5 $\mathrm{m}^{3}$ - nicht über $50 \%$ an. Zwieselförmige Buchen hingegen zeichnen sich durch einen stark wachsenden Zusammenhang aus, wobei bei einem Baumvolumen unter einem $\mathrm{m}^{3}$ ein Umgreifen nur selten erforderlich ist. Mit steigendem Baumvolumen nimmt diese Wahrscheinlichkeit zu, bis ein Umgreifen ab $2 \mathrm{~m}^{3}$ fast immer erforderlich wird. Ist die Krone besenförmig, so besteht auch bei geringem Baumvolumen eine hohe Wahrscheinlichkeit, dass innerhalb der Aufarbeitung ein Umgreifen des Aggregates notwendig wird. Zusammenfassend lässt sich ableiten: Mit steigendem Baumvolumen und bei einer besen- oder zwieselförmigen Kronenausprägung steigt die Wahrscheinlichkeit eines notwendigen Umgreifens bei der Aufarbeitung der Buche an (Formel 13).

$$
\operatorname{logit}(\hat{P}(Y \leq 1))=-2,25+0,61 * \operatorname{Vol}+1,44 * K T_{2}+2,21 * K T_{3}
$$

Formel 13: Logistische Regression der Dummy-codierten Variablen Umgreifen. $N=460$; $A I C=379$.

Weiterhin war im Rahmen der Analyse von Interesse, wie oft ein Umgreifen erfolgen muss, um den Baum in Gänze aufzuarbeiten. Aus diesem Grund wird in Abbildung 30 ein Boxplots für die Anzahl an Umgreifvorgängen pro Baum in Abhängigkeit zum Kronentyp dargestellt.

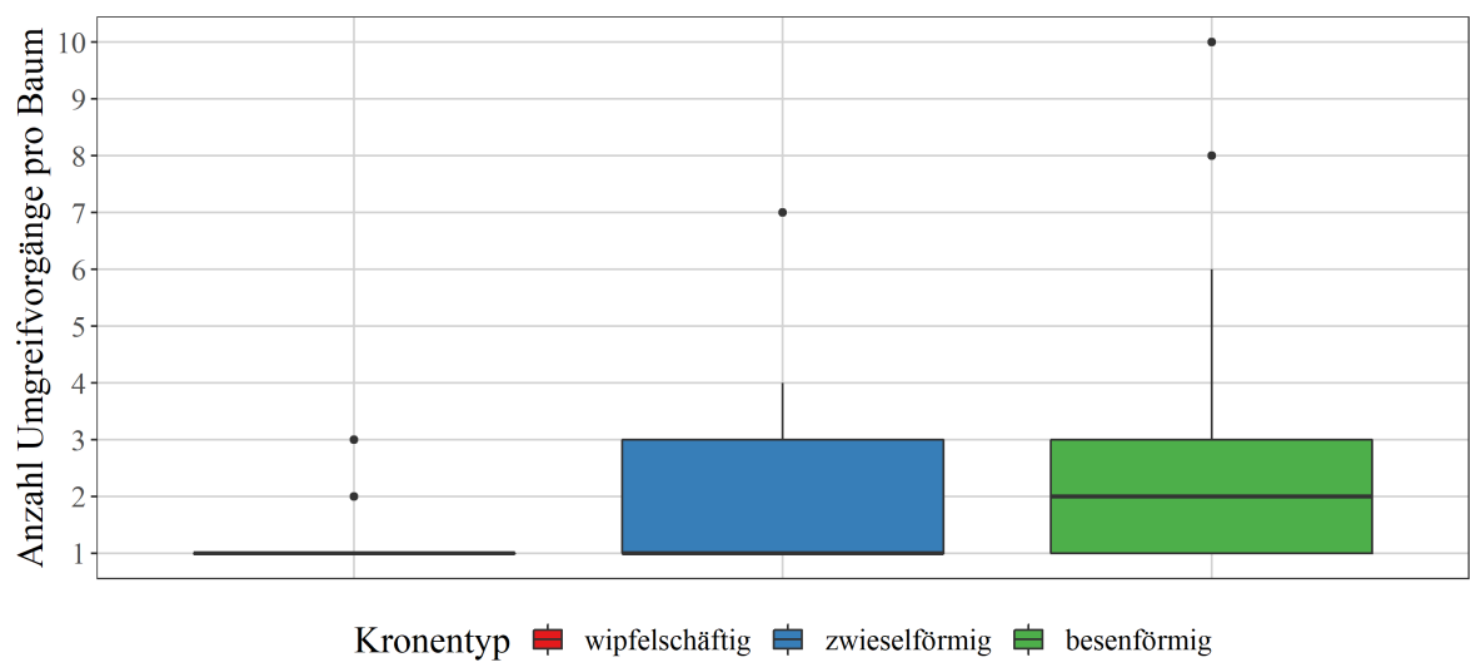

Abbildung 30: Boxplot der Umgreifvorgänge pro Baum in Abhängigkeit vom ausgebildeten Kronentyp. $N_{\text {total }}=134 ; N_{\text {wipfelschäftig }}=27 ; N_{\text {zwieselförmig }}=25 ; N_{\text {besenförmig }}=82$. 
Es wird sichtbar, dass der Kronentyp nicht nur die Wahrscheinlichkeit des Umgreifens, sondern auch die Häufigkeit dieser Aktivität beeinflusst. Bei wipfelschäftigen Buchen reicht ein einmaliges Umgreifen in der Regel aus, um diesen vollständig aufzuarbeiten. Hat der Baum jedoch eine zwiesel- oder besenförmige Krone ausgebildet, muss der Maschinenführer in vielen Fällen mehrfach umgreifen. Bei der Modellierung dieses Zusammenhanges muss beachtet werden, dass es sich bei der Anzahl an Umgreifvorgängen nicht um eine kategoriale, sondern um eine ordinal-skalierte Variable handelt. Aus diesem Grund wird anstatt einer multinominal logistischen eine geordnete logistische Regression durchgeführt (Formel 14).

$$
\begin{aligned}
& \operatorname{logit}(\hat{P}(Y \leq 1))=3,73-1,7 * \mathrm{Vol}-0,77 * K T_{2}-2,34 * K T_{3} \\
& \operatorname{logit}(\hat{P}(Y \leq 2))=5,06-1,7 * \mathrm{Vol}-0,77 * K T_{2}-2,34 * K T_{3} \\
& \operatorname{logit}(\hat{P}(Y \leq 3))=6,44-1,7 * \mathrm{Vol}-0,77 * K T_{2}-2,34 * K T_{3}
\end{aligned}
$$

Formel 14: Geordnete logistische Regression der Anzahl an Umgreifvorgängen je Baum. Dargestellt für die ersten vier Aktivitätswiederholungen. $N=134 ; A I C=257$.

Den Abschluss bildet die Betrachtung der benötigten Zeit für die Aktivität Umgreifen. In Abbildung 31 wird diese in Abhängigkeit zum Baumvolumen und der Anzahl an Umgreifvorgängen je Baum abgetragen. 

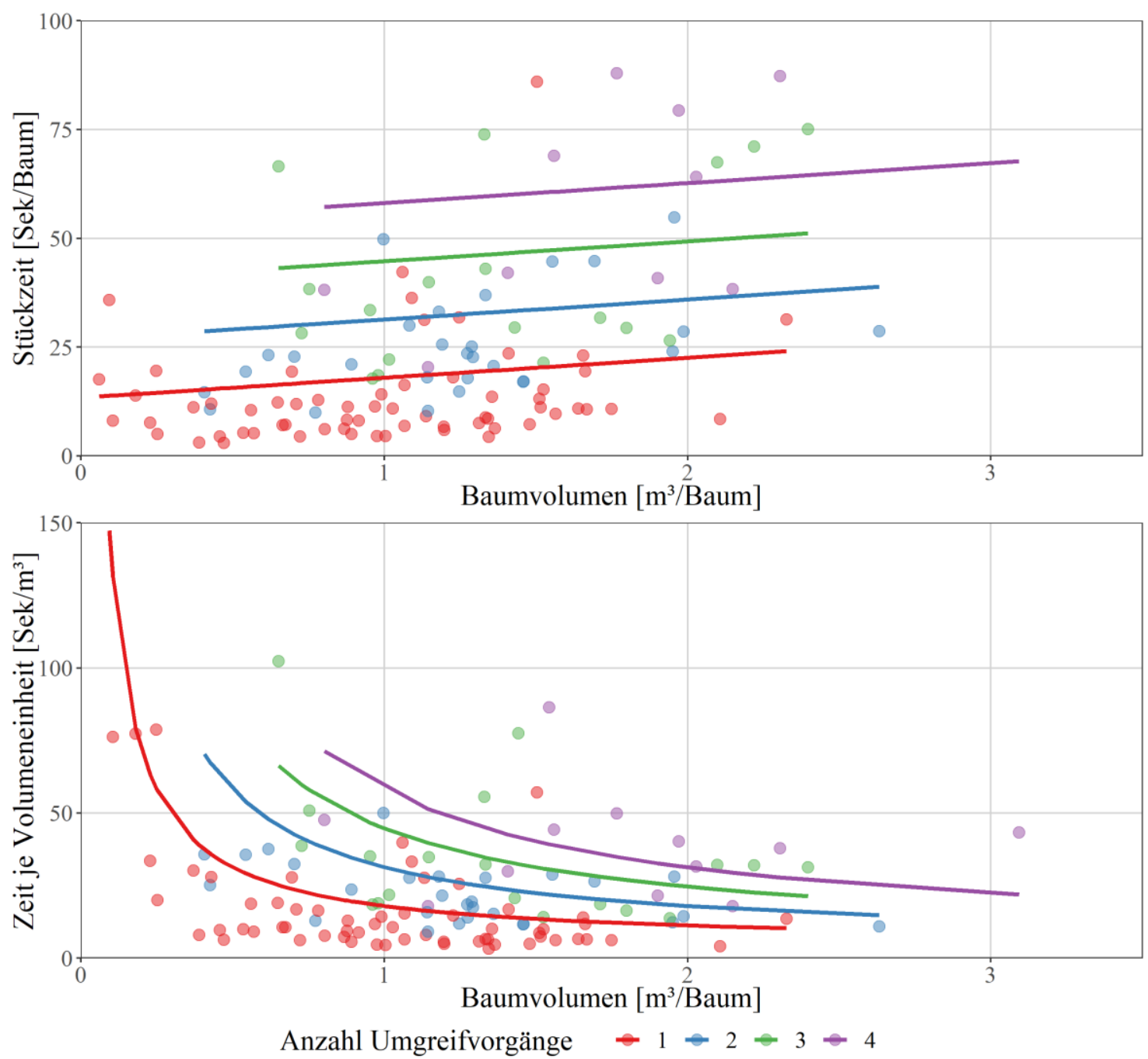

Abbildung 31: Oben: Stückzeit für die Aktivität Umgreifen in linearer Abhängigkeit vom Baumvolumen. Unten: Zeit je $\mathrm{m}^{3}$ für die Aktivität Umgreifen in Abhängigkeit vom Baumvolumen. Die Zusatzvariable beschreibt die Anzahl an Umgreifaktivitäten. Dargestellt für Bäume mit maximal vier Umgreifvorgängen. $N=134 ; R^{2}=0,67$.

Es wird sichtbar, dass die Stückzeiten über dem Baumvolumen je Anzahl an Umgreifvorgängen parallel und mit gleichem Abstand zueinander verlaufen. Jeder Umgreifvorgang benötigt also eine feste Zeit. Einen zusätzlichen Einfluss auf das Umgreifen beschreibt das Baumvolumen - der benötigte Zeitbedarf steigt demnach mit größer werdendem Baumvolumen an. Der wichtigste Kostentreiber ist aber die Anzahl an Umgreifvorgängen, die während der Aufarbeitung notwendig sind. Hinzu kommt ein Unterschied zwischen den Harvestersystemen: Während die ersten beiden Systeme relativ ähnlich umgriffen, benötigt System 3 eine erheblich längere Zeit für diese Aktivität. Eine genaue 
Übersicht über das Aktivitätsmodell zeigt Formel 15 mit den statistischen Zusatzinformationen in Tabelle 15. Eine Übersicht über alle getesteten Variablen gibt Anhang 10.

$$
\begin{aligned}
& f(\text { Umgreifen })=\beta_{\text {Vol }} * \text { Vol }+\beta_{\text {Umgreifvorgänge }} * \text { Umgreifvorgänge } \\
&+\beta_{\text {System } 1} * \text { System }_{1}+\beta_{\text {System } 2} * \text { System }_{2} \\
&+\beta_{\text {System } 3} * \text { System } \\
& 3
\end{aligned}
$$

Formel 15: Lineare Funktion zur Erklärung des Zeitverbrauchs (Sekunden/Baum) für die Aktivität Umgreifen. Legende siehe Variablenverzeichnis. $N=134 ; R^{2}=0.88$.

Tabelle 15: Regressionskoeffizient $b$, Standardfehler $S E$ und Signifikanzwert $p$ für die lineare Regression des Zeitverbrauchs (Sekunden/Baum) der Aktivität Umgreifen. Legende siehe Variablenverzeichnis. $N=134 ; A I C=1.141$.

\begin{tabular}{lccc}
\hline Faktor & $\mathbf{b}$ & $\mathbf{S E}$ & $\mathbf{p}$ \\
\hline$\beta_{\text {Vol }}$ & 10,89 & 2,81 & $<, 001^{* * *}$ \\
$\beta_{\text {Umgreifvorgänge }}$ & 13,71 & 1,08 & $<, 001^{* * *}$ \\
$\beta_{\text {System } 1}$ & $-16,85$ & 3,61 & $<, 001^{* * *}$ \\
$\beta_{\text {System } 2}$ & $-21,07$ & 4,20 & $<, 001 * * *$ \\
$\beta_{\text {System } 3}$ & 3,42 & 3,92 &, 384 \\
\hline
\end{tabular}

\subsubsection{Kronenrest}

Den Abschluss der Betrachtung der einzelnen Aktivitätsmodelle bildet die Analyse des Ablegens des Kronenrestes. Neben dem Baumvolumen und dem Kronentyp wurden weitere Variablen in die Analyse mit einbezogen (Anhang 11). Dazu zählt beispielsweise auch die Zusatzaktivität, bei welcher der Kronenrest zum Bodenschutz auf der Rückegasse abgelegt wird.

Basierend auf dem Forschungsstand (Kapitel 4.1) war davon auszugehen, dass insbesondere der Kronentyp die benötigte Zeit für das Ablegen des Kronenrestes beeinflusst. Zunächst bedarf es jedoch einer Prüfung, in welchem Zusammenhang die Stammqualität und der ausgebildete Kronentyp zueinanderstehen. Aus diesem Grund wird zunächst der Zusammenhang zwischen dem Kronentyp und der Qualität des Baumes in Abhängigkeit zu dessen Baumvolumen näher betrachtet (Abbildung 32). 

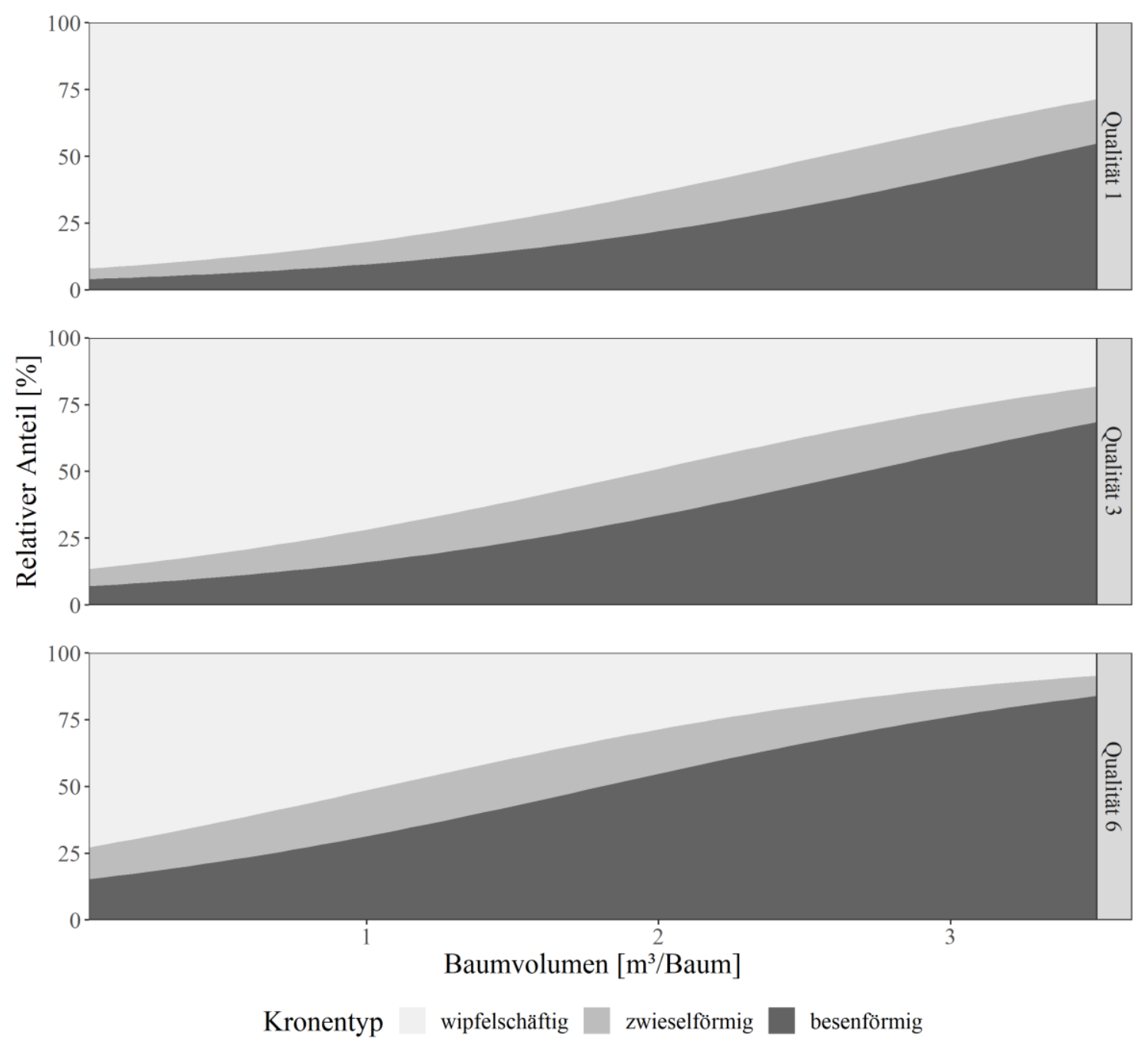

Abbildung 32: Modellierte relative Anteile des Kronentyps in Abhängigkeit zum Baumvolumen und der Qualität. Dargestellt für die Qualitäten 1,3 und 6; $N=181$.

Es zeigt sich, dass Bäume mit schlechterer Qualität häufiger besenförmige Kronentypen aufweisen, wohingegen Bäume mit guter Qualität häufiger wipfelschäftige Kronentypen ausgebildet haben. Obwohl dies auf eine Wechselwirkung zwischen der Qualität des Baumes und dem Kronentyp hinweist - je schlechter die Qualität des Baumes, desto geringer ist der Anteil an wipfelschäftigen Individuen - konnte in der Modellierung keine signifikante Wechselwirkung nachgewiesen werden. Darüber hinaus wird erkenntlich, dass mit steigendem Baumvolumen der Anteil an wipfelschäftigen Buchen, unabhängig der Qualität, immer geringer wird.

Abbildung 33 zeigt den Zeitverbrauch der eigentlichen Aktivität des Kronenrestablegens. Hierzu wird die Bearbeitungszeit pro Baum sowie pro $\mathrm{m}^{3}$ in Abhängigkeit zum Baumvolumen und zum ausgebildeten Kronentyp dargestellt. 

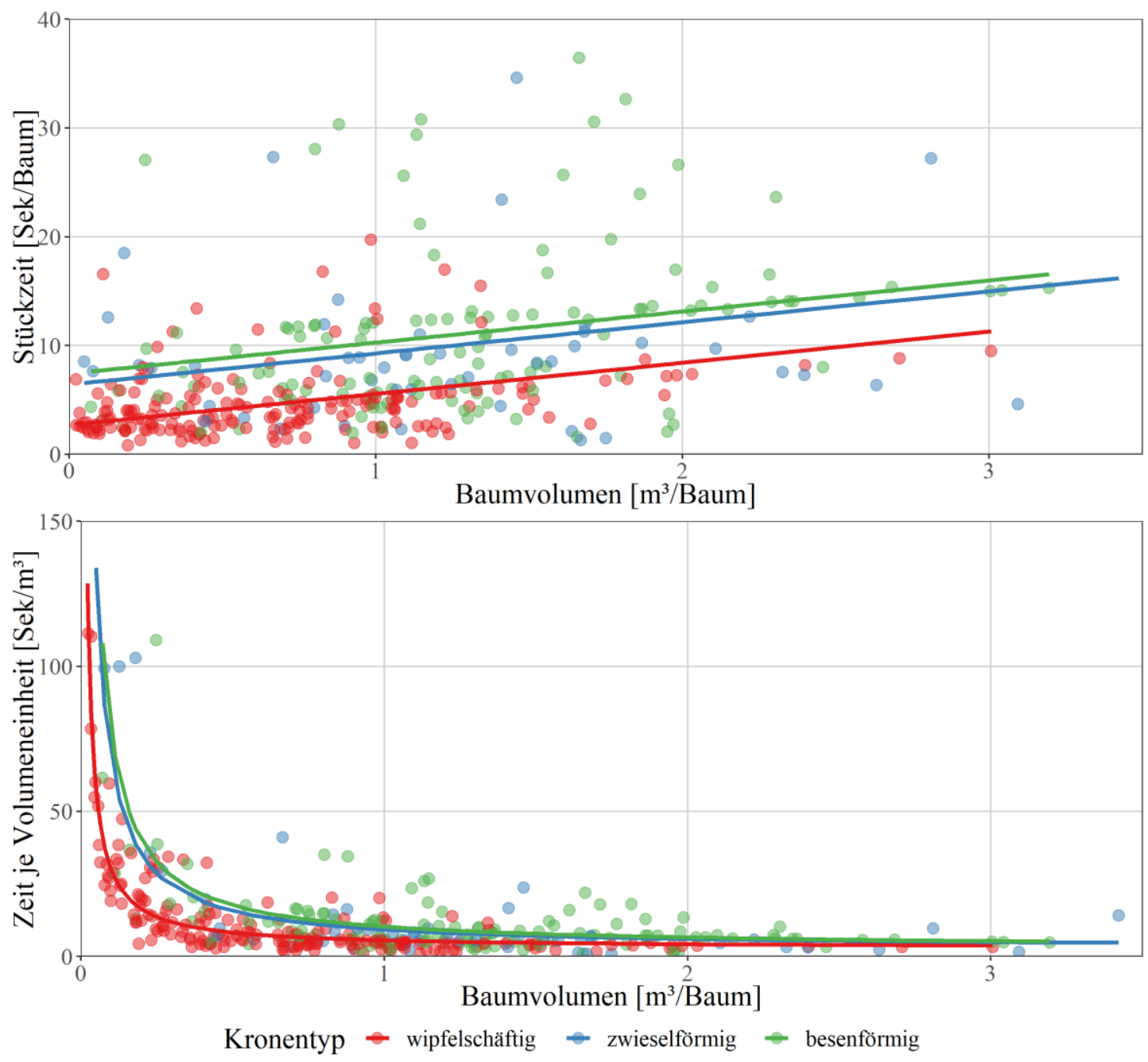

Abbildung 33: Oben: Stückzeit für die Aktivität Kronenrest ablegen in linearer Abhängigkeit vom Baumvolumen. Unten: Zeit je $\mathrm{m}^{3}$ für die Aktivität Kronenrest ablegen in Abhängigkeit vom Baumvolumen. Die Zusatzvariable beschreibt den Einfluss des Kronentyps auf die abhängige Variable. $N=361 ; R^{2}=0.28$.

Es wird sichtbar, dass die benötigte Zeit pro Baum mit steigendem Baumvolumen zunimmt. Ebenfalls kann die Forschungshypothese H3 über den erwarteten Einfluss des Kronentyps mit diesem linearen Modell bestätigt werden. Bei einer zwieselförmigen Buche steigt die benötigte Zeit um fast drei Sekunden gegenüber einem wipfelschäftigen Individuum an; bei einer besenförmiger Kronenausbildung beträgt die Mehrzeit sogar rund fünf Sekunden. Eine Übersicht über das Modell gibt Formel 16 mit der Übersicht der statistischen Kennziffern in Tabelle 16. 
Ergebnisse

$$
\begin{aligned}
f(\text { Kronenrest }) & =\beta_{0}+\beta_{V o l} * \text { Vol }+\beta_{\text {ZusatzK1 } 1} * \text { Zusatz }_{1} \\
& +\beta_{K T 2} * K T_{2}+\beta_{K T 3} * K T_{3}
\end{aligned}
$$

Formel 16: Lineare Funktion des Zeitverbrauchs (Sekunden/Baum) für das Ablegen des Kronenrestes. Legende siehe Variablenverzeichnis. $N=361 ; R^{2}=0.34$.

Tabelle 16: Regressionskoeffizient $b$, Standardfehler $S E$ und Signifikanzwert $p$ für die lineare Regression des Zeitverbrauchs (Sekunden/Baum) der Aktivität Kronenrest ablegen. Legende siehe Variablenverzeichnis. $N=361 ; A I C=2.249$.

\begin{tabular}{lccc}
\hline Faktor & b & SE & p \\
\hline$\beta_{0}$ & 2,83 & 0,51 & $<, 001^{* * *}$ \\
$\beta_{\text {Vol }}$ & 2,67 & 0,47 & $<, 001^{* * *}$ \\
$\beta_{\text {ZusatzK1 }}$ & 14,37 & 2,50 & $<, 001^{* * *}$ \\
$\beta_{K T 2}$ & 2,70 & 0,91 &, $003 * *$ \\
$\beta_{K T 3}$ & 4,71 & 0,69 & $<, 001 * * *$ \\
\hline
\end{tabular}

Die Ablage des Kronenrestes kann somit als Aktivität verstanden werden, die einem relativ geringen Volumentrend folgt. Neben der Verlaufsvariablen beeinflusst hauptsächlich der Kronentyp die benötigte Zeit.

Mit der Modellierung der letzten Aktivität kann Forschungshypothese H1 bestätigt werden. Jede einzelne Aktivität konnte in einem separaten Produktivitätsmodell analytisch beschrieben und deren jeweils wichtigsten Kostentreiber identifiziert werden.

\subsection{Modellierung des Gesamtprozesses}

Die Modellierung des Gesamtzeitverbrauches erfolgte anschließend durch die Aufsummierung der einzelnen Aktivitäten (Formel 17, in Anlehnung an Nurminen et al., 2006, S. 342).

$$
f(\text { Prozess })=f(A)+f(G)+f(F)+f(E)+f(U)+f(K)
$$

Formel 17: Funktion des Gesamtprozesses durch die Summierung der Aktivitätsmodelle mit $\mathrm{A}=$ Anfahrt und Positionierung, $\mathrm{G}=$ Greifen, $\mathrm{F}=$ Fällung, $\mathrm{E}=$ Entasten, $\mathrm{U}=\mathrm{Um}$ greifen und $\mathrm{K}=$ Kronenrest. 
Im Vergleich zur Arbeit von Nurminen et al. (2006, S. 342) zeichnet sich die vorliegende Arbeit durch zwei Besonderheiten aus: Die große Zahl an Parametern, die in das Gesamtmodell integriert sind, und deren komplexere Aufnahmemethodik. Das Gesamtmodell besteht aus 13 Variablen, die keinem einheitlichen Skalenniveau folgen. Um eine bessere Übersicht der berücksichtigten Variablen zu gewährleisten, werden in Tabelle 17 die in Kapitel 7.1 zusammengetragenen Befunde gebündelt berichtet.

Tabelle 17: Übersicht über die einbezogenen Variablen innerhalb der Aktivitätsmodelle aus Kapitel 7.1. Die mit einem X markierten Variablen besitzen einen signifikanten Einfluss auf die jeweilige Aktivität. Legende siehe Variablenverzeichnis.

\begin{tabular}{|c|c|c|c|c|c|c|}
\hline & $f(A)$ & $f(G)$ & $f(F)$ & $f(E)$ & $f(U)$ & $f(K)$ \\
\hline$\beta_{0}$ & $\mathrm{X}$ & $\mathrm{X}$ & $\mathrm{X}$ & $\mathrm{X}$ & & $\mathrm{X}$ \\
\hline$\beta_{V o l}$ & & $\mathrm{X}$ & $\mathrm{X}$ & $X$ & $\mathrm{X}$ & $\mathrm{X}$ \\
\hline$\beta_{V o l^{2}}$ & & & $\mathrm{X}$ & $\mathrm{X}$ & & \\
\hline$\beta_{\text {Entf }}$ & $\mathrm{X}$ & & & & & \\
\hline$\beta_{\text {Verlauf }}{ }^{1}$ & & $\mathrm{X}$ & $\mathrm{X}$ & & & $\mathrm{X}$ \\
\hline$\beta_{K T}$ & & & & & $X^{2}$ & $\mathrm{X}$ \\
\hline$\beta_{\text {Qualität }}$ & & & $X$ & $\mathrm{X}$ & & \\
\hline$\beta_{\text {Abschnitte }}$ & & & & $\mathrm{X}$ & & \\
\hline$\beta_{\text {Sorten }}$ & & & & $\mathrm{X}$ & & \\
\hline$\beta_{\text {Umgreifvorgänge }}$ & & & & & $\mathrm{X}$ & \\
\hline$\beta_{\text {System }}$ & & $\mathrm{X}$ & $\mathrm{X}$ & & $\mathrm{X}$ & \\
\hline
\end{tabular}

${ }^{1}$ Zusammenfassung der Dummy-codierten Verlaufs- und Verursachungsvariablen

${ }^{2}$ Der Kronentyp hat einen indirekten Einfluss über die Wahrscheinlichkeit des Umgreifens und die Anzahl an Umgreifvorgängen

Daraus geht hervor, dass das Baumvolumen als ein zentraler Einflussfaktor identifiziert werden konnte, welcher mit Ausnahme der Anfahrt und Positionierung auf alle anderen Aktivitäten einen signifikanten Einfluss ausübt. Es gibt aber auch eine Vielzahl an Kostentreibern, wie beispielsweise die Entfernung, die jeweils nur eine einzelne Aktivität beeinflussen. Bemerkenswert ist auch der Effekt des eingesetzten Harvestersystems, da dieser nicht bei allen Aktivitäten festgestellt werden konnte. Das kumulierte Modell zur Ergänzung der Tabelle 17 zeigt Formel 18. 
Ergebnisse

$$
\begin{aligned}
& f(\text { Gesamt })=11,87+43,61 * V o l+9,78 * V_{o l}{ }^{2}+0,44 * E n t f \\
& +9.93 * \text { ZusatzG1 }+4,41 * S 1 \\
& +7,5 * Z 1+12,22 * L 1+14,37 * \text { ZusatzK } 1 \\
& +2,7 * K T_{2}+4,71 * K T_{3}+3,3 * \text { Qualität } \\
& +1,56 * \text { Abschnitte }+5,26 * \text { Sorten } \\
& +13,71 *{\text { Umgreifvorgänge }-16,85 * \text { System }_{1}} \\
& -25,34 * \text { System }_{2}+13,43 * \text { System }_{3}
\end{aligned}
$$

Formel 18: Kumuliertes Gesamtmodell (Sekunden/Baum) für die mechanisierte Aufarbeitung von Buche. Legende siehe Variablenverzeichnis.

Nun wird sichtbar, dass das Baumvolumen zusätzlich auch der stärkste Kostentreiber des Gesamtprozesses ist (Anhang 12). Danach scheint das eingesetzte Harvestersystem den zweitstärksten Einfluss auszuüben, gefolgt von der Anzahl an Umgreifvorgängen sowie der Anzahl ausgehaltener Sortimente.

Durch die Aufsummierung der Modelle ist eine Aussage zur Güte der Gesamtfunktion in Form eines statistischen Parameters (bspw. AIC oder $R^{2}$ ) nicht ohne Weiteres möglich. Um die Qualität des Gesamtmodells zu überprüfen, erfolgt daher eine Gegenüberstellung der vorhergesagten und der beobachteten Werten für den Gesamtprozess (Abbildung 34).

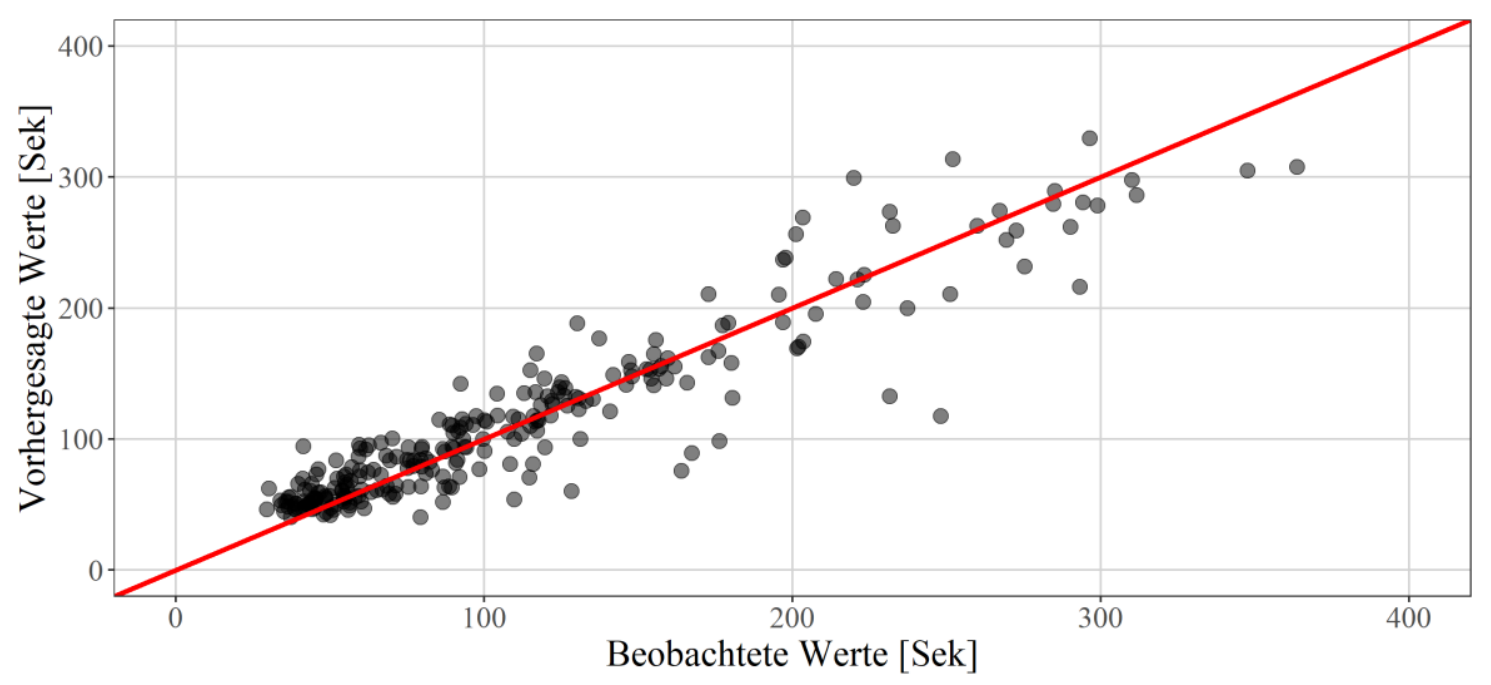

Abbildung 34: Vergleich der beobachteten und der vorhergesagten Werte. Die vorhergesagten Werte wurden durch die Anwendung des vorgestellten Gesamtmodells berechnet (Formel 18). Die rote Linie zeigt die Winkelhalbierende, bei der das Residuum $=0$ beträgt. $N=254$. 
Es fällt auf, dass das Modell eine Homoskedastizität aufweist. Die Verteilung der Residuen ist nahezu normalverteilt und unterstreicht damit die Güte und insbesondere die Validität des Modells (Anhang 13). Das summierte Gesamtprozessmodell ist zweifelsfrei zur Beschreibung des gesamten Aufarbeitungsprozesses geeignet, obwohl es nicht direkt angepasst wurde, sondern sich aus den Aktivitätsmodellen zusammensetzt. Die Quadratwurzel des gemittelten Fehlerquadrats (RMSE), also die mittlere Standardabweichung des Gesamtmodells, beträgt 25,65 Sekunden.

Zusammenfassend kann somit darauf geschlossen werden, dass das Modell den Prozess der mechanisierten Buchenaufarbeitung im Rahmen des vorhandenen Datensatzes korrekt widerspiegelt und dabei auch bemerkenswert genau ist. Ein Problem des Modells ist allerdings seine Praktikabilität: Neben der Überprüfung der Forschungshypothesen auf theoretischer Ebene sollen die Modelle auch für die Praxis nutzbar gemacht werden, um den Zeitbedarf der mechanisierten Buchenaufarbeitung zu schätzen. Da einige Modellvariablen allerdings zu abstrakt sind, um mit vertretbarem Aufwand gemessen werden zu können, ist das summierte Modell in seiner jetzigen Form zu komplex. Aus diesem Grund wird das Modell nachfolgend gekürzt, indem abstrakte Variablen eliminiert werden. Da die Reduktion von Modellbestandteilen die Genauigkeit obligatorisch verschlechtert, muss hierbei stets abgewogen werden, welche Variablen eliminiert werden können, ohne die Genauigkeit zu stark einzuschränken.

\subsubsection{Analytische Variablenreduktion}

Das Kürzen des Modells erfolgt, indem die Terme der jeweiligen Variablen aufgelöst werden. Die mathematischen Grundlagen und Beweise dieses Vorgehens wurden unter anderem von Rencher und Schaalje (2008, S. 93-99) beschrieben. Die mittlere Ausprägung der Variablen, berechnet aus dem Parametrisierungsdatensatz, wird in das Modell eingesetzt und durch die Anzahl der Beobachtungen geteilt. Damit kann der Term ausgerechnet und mit dem y-Achsenabschnitt verrechnet werden. Die aufgelösten Variablen werden somit unter einem aktualisierten konstanten Wert $\beta_{0}$ zusammengefasst.

Es wurde sich dazu entschieden, nur Variablen im reduzierten Modell zu belassen, die auch in der Praxis relativ leicht gemessen oder eingeschätzt werden können. Dazu gehören neben dem Einzelbaumvolumen auch die Entfernung, Qualität, der Kronentyp und 
Ergebnisse

die Anzahl an Sortimenten, die ausgehalten werden sollen. Alle anderen Variablen werden aufgelöst und zu der Konstanten addiert. Formel 19 zeigt die reduzierte Gesamtformell.

$$
\begin{aligned}
f(\text { Gesamt }) & =36,6+43,61 * \text { Vol }+9,78 * V_{o l}^{2}+0,44 * \text { Ent }_{\text {Baum }} \\
& +3,3 * \text { Qualität }+5,26 * \text { Sorten } \\
& +2,70 * K T_{2}+4,71 * K T_{3}
\end{aligned}
$$

Formel 19: Reduziertes Gesamtmodell (Sekunden/Baum) für die mechanisierte Aufarbeitung von Buche. Legende siehe Variablenverzeichnis.

Wie auch beim ungekürzten Modell, kann die Güte des reduzierten Modells nicht über $A I C$ - oder $R^{2}$-Werte angegeben werden. Aus diesem Grund erfolgt im ersten Schritt erneut ein Vergleich der beobachteten und der vorhergesagten Werte (Abbildung 35).

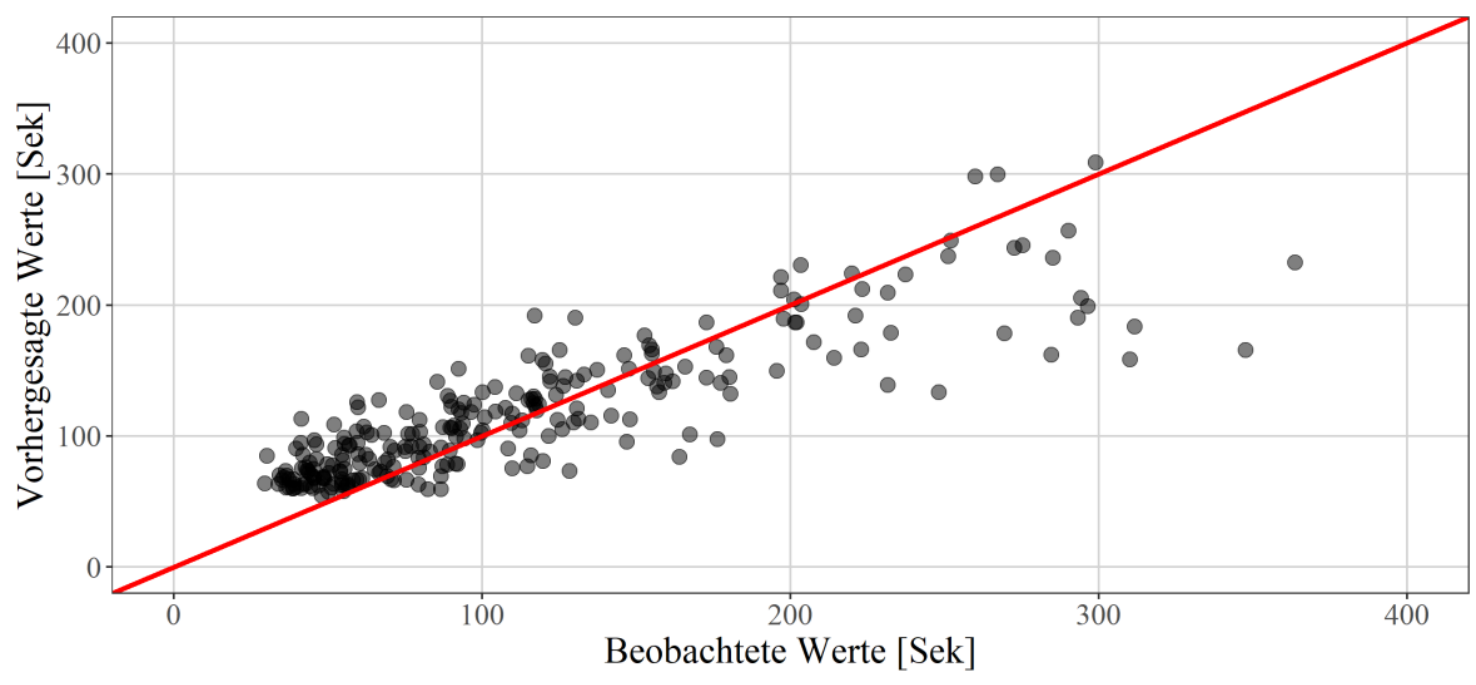

Abbildung 35: Vergleich der beobachteten und der vorhergesagten Werte. Die vorhergesagten Werte wurden durch die Anwendung des reduzierten Gesamtmodells berechnet (Formel 19). Die rote Linie zeigt die Winkelhalbierende, bei der die Abweichung $=0$ beträgt. $N=254$.

Im Vergleich zum Gesamtmodell (Abbildung 34) kommt es beim reduzierten Modell zu erwartbaren Veränderungen zwischen der Beobachtung und der Vorhersage: Aufgrund des erhöhten y-Achsenabschnitts von rund 25 Sekunden überschätzt das reduzierte Modell den notwendigen Zeitverbrauch im unteren Bereich der beobachteten Werte. Bei beobachteten Werten über 150 Sekunden tendiert das reduzierte Modell hingegen dazu, den 
vorhergesagten Wert zu unterschätzen. Diese Unterschätzung geht vor allem auf die Reduktion der vielen Verlaufsvariablen und des Umgreifens zurück. Einzelne Eintrittswahrscheinlichkeiten der Zusatzaktivitäten (z. B. Loslassen bei der Fällung, Kapitel 7.1.3) und die Anzahl an Umgreifvorgängen pro Baum (Kapitel 7.1.5) skalieren stark mit dem Baumvolumen.

Diese Beobachtungen werden auch von der Verteilung der Residuen (Anhang 14) bestätigt - die bisher normalverteilten Residuen weisen nun eine rechtsschiefe Verteilung auf. Die Richtung der Schiefe ist aber hauptsächlich durch den verzerrten Schwerpunkt der Daten (Bias) bestimmt, der bei einem Beobachtungswert von 113 Sekunden liegt. Wie stark diese Veränderung ausfällt, zeigt die Veränderung des RMSE. Dieser steigt im Vergleich zum ungekürzten Modell um $52 \%$, also auf insgesamt 39,1 Sekunden, an.

Da die bisher vorgestellten Funktionen sich ausschließlich auf der Basis des Zeitverbrauches (Sekunden) pro Baum bewegen, soll folgend die umgeformte Funktion im Sinne der Produktivität (MAS 0 in Stunden) dargestellt werden (Formel 20).

$$
\begin{aligned}
p_{\text {Gesamt }}\left(\frac{m^{3}}{M A S_{0}}\right) & =\frac{41,61}{3600}+\frac{9,78 * \text { Vol }}{3600} \\
& +\frac{V o l * 3600}{36,6}+\frac{V o l * 3600}{0,44 * \text { Entf }}+\frac{V o l * 3600}{3,3 * \text { Qualität }} \\
& +\frac{V o l * 3600}{5,26 * \text { Sorten }}+\frac{V o l * 3600}{2,70 * K T_{2}}+\frac{V o l * 3600}{4,71 * K T_{3}}
\end{aligned}
$$

Formel 20: Produktivitätsmodell der mechanisierten Buchenaufarbeitung. Legende siehe Variablenverzeichnis.

Auch wenn die Funktion im Vergleich zum ungekürzten Modell (Formel 18) an Komplexität verliert, ist es wichtig hervorzuheben, dass es sich immer noch um ein unverzerrtes Modell handelt, das nun aber nur noch von einer geringen Anzahl an (einfach einzuschätzenden) Kostentreibern beeinflusst wird. Um die Stärke dieser Faktoren etwas genauer zu analysieren, folgt im nächsten Abschnitt eine Sensitivitätsanalyse.

\subsubsection{Einfluss der verbleibenden Kostentreiber}

Um das Randverhalten der Kostentreiber auf die Produktivität besser quantifizieren zu können, werden diese im Rahmen einer Sensitivitätsanalyse variiert. Da die Stärke der Variablen sowie auch deren Unsicherheiten bereits eingehend analysiert wurden, wird als 
Ergebnisse

Grundlage für die Sensitivitätsanalyse der bestehende Datensatz genutzt.

Für jede getestete Variablenausprägung wird hierbei eine Kopie des Datensatzes erzeugt, in dem alle Werte bis auf die betrachten Variablen unverändert bleiben. Diese wird dann mit der jeweiligen Ausprägung bei allen Entnahmebäumen überschrieben. Unter Anwendung des Produktivitätsmodells (Formel 20) kann daraus ein Produktivitätsfächer für jeden Kostentreiber berechnet werden. Anhang 15 zeigt einen solchen Fächer für verschiedene zurückgelegte Entfernungsdistanzen zum Baum während der Aufarbeitung. Um die Interpretation dieser Analyse zu erleichtern, werden nicht die absoluten Varianten, sondern deren Relation zueinander untersucht. Die Basis bildet jeweils die produktivste Variablenkombination. Abbildung 36 zeigt daher die relativen Produktivitätsdifferenzen für das Variantenstudium der Entfernung über dem Baumvolumen. Die y-Achse beschreibt die Produktivitätsdifferenz zwischen einer festgelegten Variablenausprägung und seinem Basiswert. Dieser Basiswert kann als die produktivste Variablenausprägung je Kostentreiber verstanden werden. Bei der zurückgelegten Entfernung zum Entnahmebaum entspricht dies $0 \mathrm{~m}$. Jede andere Variablenausprägung wird dann in Relation zu dieser Basis gesetzt und ergibt somit einen Fächer der Produktivitätsdifferenzen. Die Darstellung dieses Fächers erfolgt dabei stets in Abhängigkeit zum Baumvolumen.

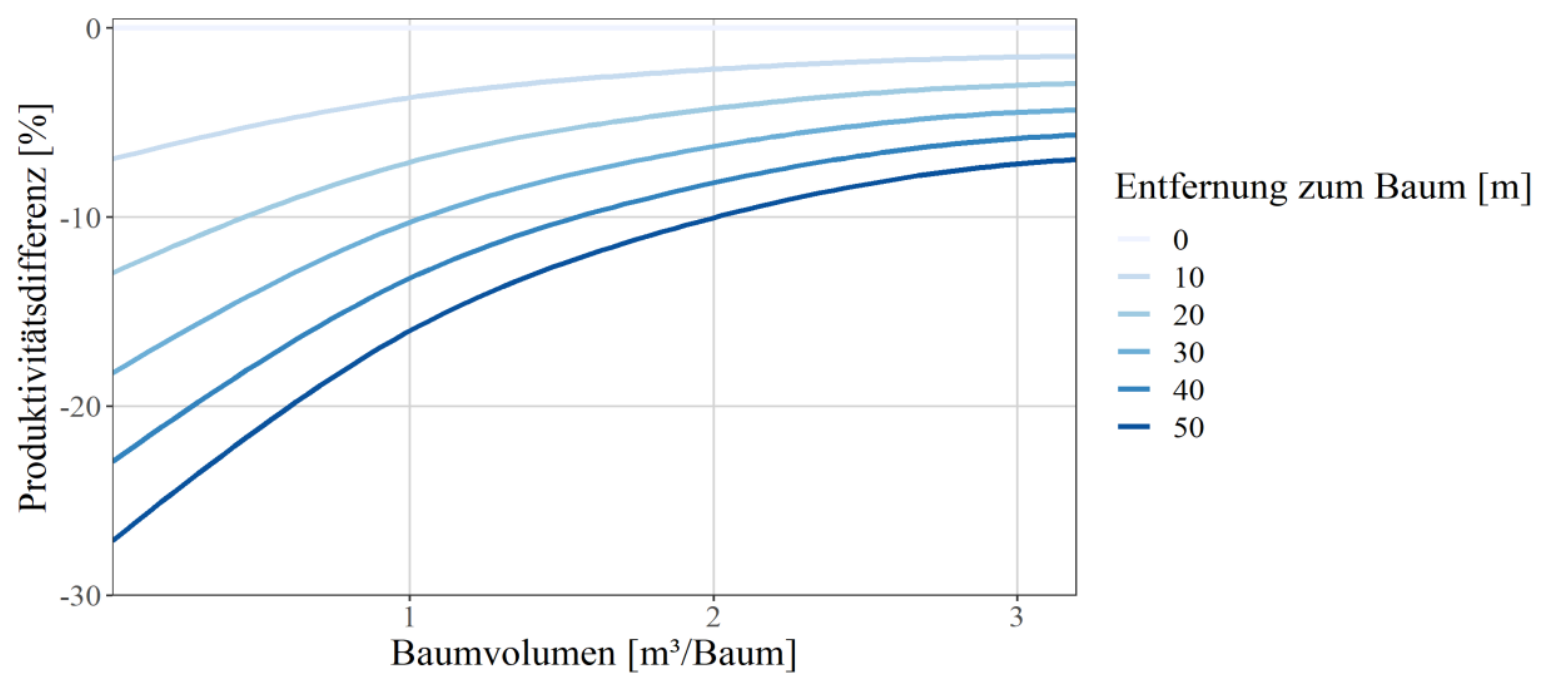

Abbildung 36: Relative Produktivitätsdifferenzen bei Anwendung des Produktivitätsmodells (Formel 20) in Abhängigkeit zum Baumvolumen und der Baumentfernung mit der Basis: Entfernung zum Baum $0 \mathrm{~m}=100 \%$. 
Es zeigt sich ein starker Einfluss der Entfernung auf die Produktivität. Bei einem Baumvolumen von $0,5 \mathrm{~m}^{3}$ wird die Produktivität bereits um $10 \%$ reduziert, sobald der Harvester 20 m zurücklegen muss. Zusätzlich zeigt sich, dass eine genaue Aussage nur in Relation zum Baumvolumen erfolgen kann. Durch den zunehmenden Einfluss des Baumvolumens sinkt der Einfluss der Variablen mit wachsender Baumstärke. Dies lässt sich auch bei den anderen leistungsbeeinflussenden Faktoren beobachten (Abbildung 37).

Die Qualitätseinteilung weist eine ebenso starke Spannweite auf die Produktivität auf, wie bereits die Entfernung zum Baum (0-50 m) gezeigt hat. Das Produktivitätsmodell (Formel 20) lässt dabei bereits erahnen, dass die Anzahl der Sortimente und der Kronentyp die Produktivität nur vergleichsweise gering beeinflussen. Mit Ausnahme der Produktivitätsdifferenz der Sortenvielfalt (Abbildung 37, Mitte), zeigen die anderen Sensitivitätsabbildungen alle möglichen Variablenkombinationen und damit auch den Extrapolationsbereich der Ausprägungen. Zu beachten ist hierbei, dass die Differenzverläufe für die Anzahl der ausgehaltenen Sortimente gekürzt dargestellt werden. Jede Kurve beginnt mit dem Baumvolumen, bei welchem die jeweilige Variablenausprägung innerhalb der Untersuchung festgestellt wurde. Alle Kostentreiber vereint aber die sinkende relative Einflussstärke mit steigendem Baumvolumen. 
Ergebnisse
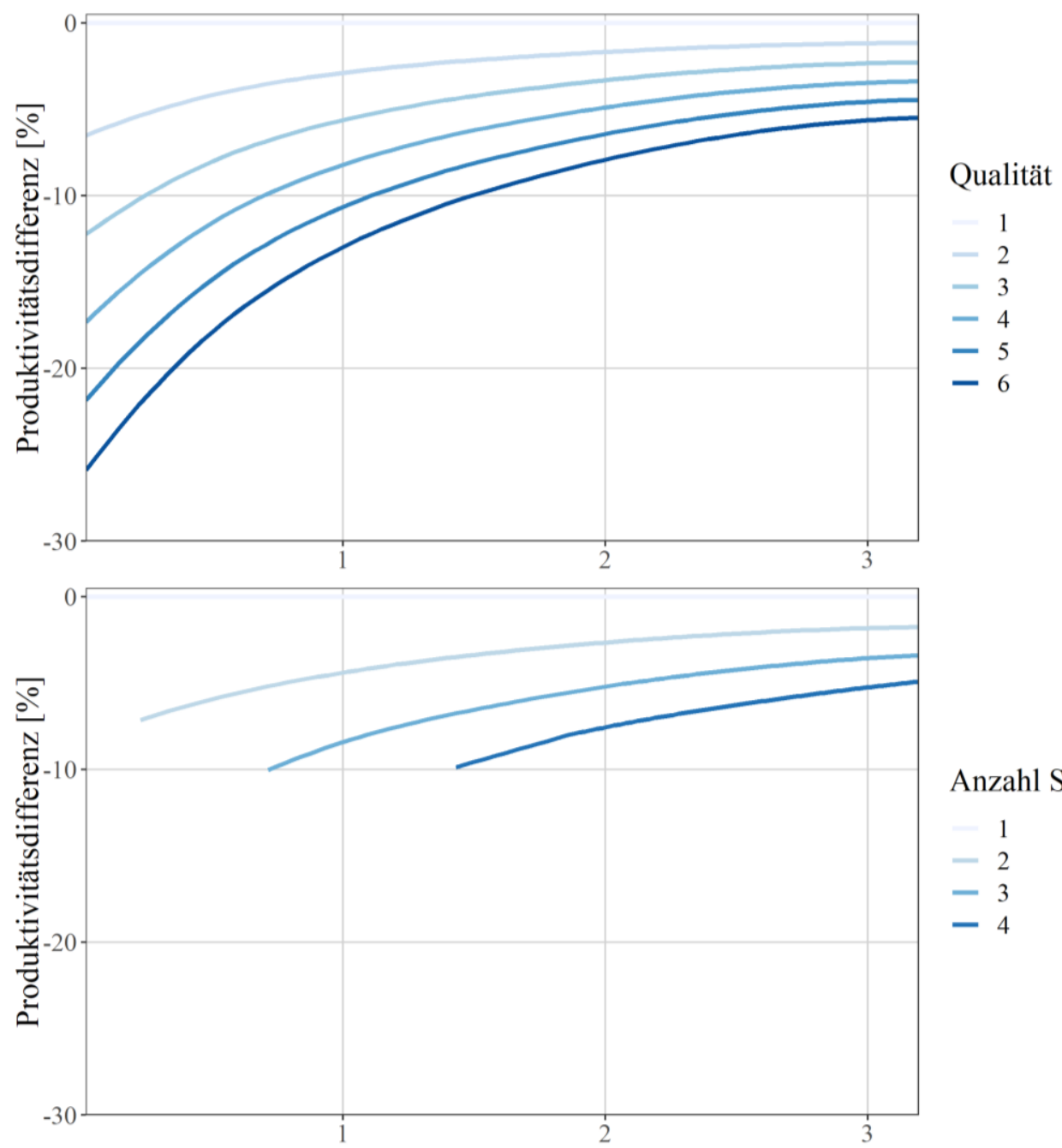

Anzahl Sortimente

1
$-\quad 2$
$-\quad 3$
-4

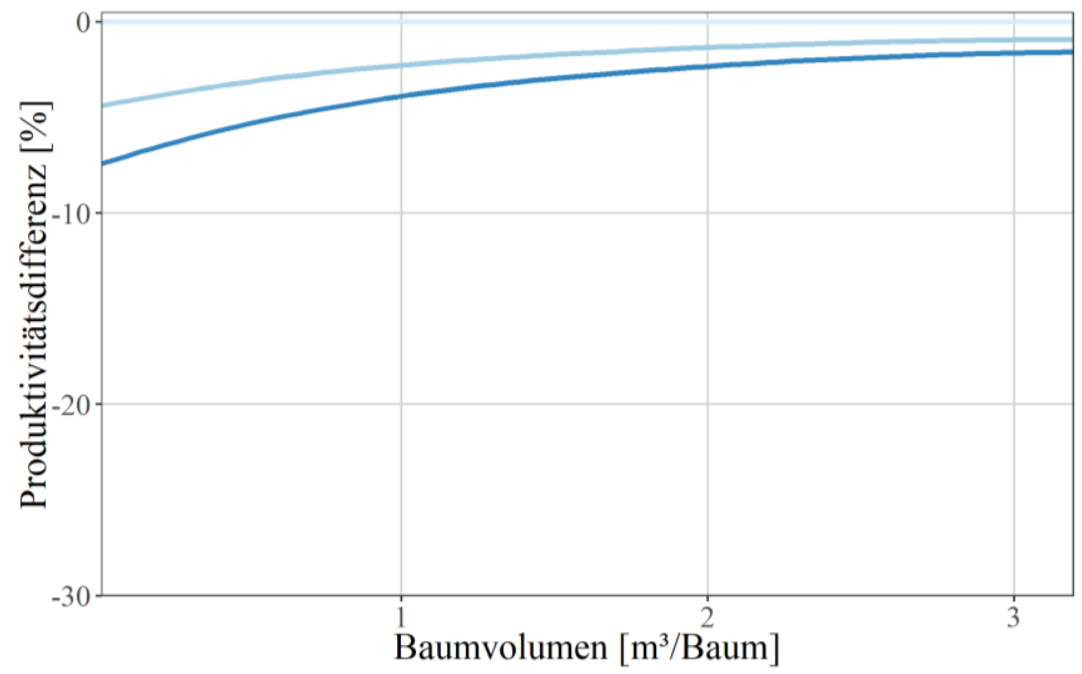

Kronentyp

- wipfelschäftig

- zwieselförmig

- besenförmig

Abbildung 37: Relative Produktivitätsdifferenzen bei Anwendung des Produktivitätsmodells (Formel 20) in Abhängigkeit der Qualitätsstufe (Oben) mit der Basis: Qualität $1=100 \%$, der Anzahl ausgehaltener Sortimente (Mitte) mit der Basis: Anzahl Sortimente $1=100 \%$ und des Kronentyps (Unten) mit der Basis: Kronentyp „,wipfelschäftig“" $=100 \%$. 


\subsubsection{Anwendung auf Bestandesebene}

Im nächsten Schritt erfolgt die Anwendung des Produktivitätsmodells (Formel 20) auf Bestandesebene, um die Frage zu beantworten, ob die Modellergebnisse realistisch auf die Fläche hochgerechnet werden können. Die Durchführung lässt sich auf zwei unterschiedliche Weisen realisieren. Zum einen kann das Modell auf einen mittleren Baum angewendet werden, welcher sich aus den durchschnittlichen Baumattributen eines Bestandes berechnet (z. B. Durchmesser und Höhe des Grundflächenmittelstammes, häufigste Qualität, etc.). Auf Bestandesebene wird folglich die mittlere Ausprägung aller Kostentreiber berechnet oder geschätzt und das Produktivitätsmodell angewendet. Das Ergebnis zeigt die mittlere Produktivität der mechanisierten Aufarbeitung innerhalb des Bestandes. Dies ermöglich eine einfache und schnelle Anwendung des Modells dieser Arbeit.

Zum anderen kann das Modell direkt auf alle Einzelbaumdaten eines Bestandes angewendet werden, was in der Regel zu einer besseren Genauigkeit bei der Einschätzung der Produktivität führt, da im Speziellen nur die zur Entnahme geplanten Einzelbäume mit ihren jeweiligen Ausprägungen berücksichtigt werden. Da die Sensitivität der Variablen bisher nur ceteris paribus separat betrachtet werden konnte, die Abhängigkeiten der Variablen untereinander jedoch auch von praktischem Interesse sind, wird in diesem Unterkapitel das Verhalten des Gesamtmodells noch einmal getestet, indem es im Rahmen einer Fallstudie auf realistische Bestände angewendet wird. Als Grundlage für die Testbestände wurden die Ergebnisse der dritten Bundeswaldinventur genutzt (BMEL, 2020), aus denen im Anschluss Bestände modelliert wurden. Tabelle 18 zeigt die forstlichen Parameter getrennt nach Altersklassen für mittlere Buchenreinbestände laut BWI. 
Tabelle 18: Ergebnisse der dritten Bundeswaldinventur für Buchenbestände getrennt nach Altersklasse (rechnerischer Reinbestand).

\begin{tabular}{lccccc}
\hline Altersklasse & $\begin{array}{c}\text { BHD } \\
{[\mathrm{cm}]}\end{array}$ & $\begin{array}{c}\text { Höhe } \\
{[\mathrm{m}]}\end{array}$ & $\begin{array}{c}\text { Volumen } \\
{[\text { Vfm/Baum }]}\end{array}$ & $\begin{array}{c}\text { Grundfläche } \\
{\left[\mathrm{m}^{2} / \mathrm{ha}\right]}\end{array}$ & $\begin{array}{c}\text { Stammzahl } \\
{[\mathrm{N} / \mathrm{ha}]}\end{array}$ \\
\hline 21 - 40 Jahre & 12,64 & 9,86 & 0,08 & 22,23 & 1771 \\
41 - 60 Jahre & 19,64 & 14,90 & 0,29 & 28,71 & 948 \\
61 - 80 Jahre & 25,95 & 21,37 & 0,61 & 30,66 & 580 \\
81 - 100 Jahre & 32,73 & 25,12 & 1,12 & 30,88 & 367 \\
101 - 120 Jahre & 39,44 & 28,09 & 1,78 & 31,16 & 255 \\
121 - 140 Jahre & 43,94 & 29,93 & 2,32 & 30,19 & 199 \\
141 - 160 Jahre & 49,11 & 31,03 & 2,98 & 28,29 & 149 \\
$>$ 160 Jahre & 54,25 & 31,43 & 3,73 & 27,65 & 120 \\
alle Altersklassen & 26,59 & 31,75 & 0,73 & 27,21 & 490 \\
\hline
\end{tabular}

Durch die Eingabe der Parameter für Baumart, Alter, Höhe, Durchmesser des Grundflächenmittelstammes und der Grundfläche wird mithilfe des Java-Tools von Nagel und Biging (1995) eine Durchmesserverteilung erzeugt und Durchmesser sowie Höhen geschätzt. ${ }^{47}$ Das Produktivitätsmodell wird im Rahmen dieser Fallstudie auf alle Bäume der virtuellen Bestände angewendet. Dementsprechend werden keine Entnahmebäume definiert, sondern alle Bäume geerntet. So ist eine Ergebnisverzerrung durch eine waldbaulich gesteuerte Auswahl der Entnahmebäume ausgeschlossen.

Da nicht alle nötigen Variablen des Produktivitätsmodells direkt aus den BWI-Daten ablesbar sind, müssen diese im nächsten Schritt über eigene Modelle geschätzt und ergänzt werden. Der erste herzuleitende Faktor ist das Baumvolumen, da nur das Einzelbaumvolumen in Vfm bekannt ist. In vielen wissenschaftlichen Berichten wird der Faktor von 0,8 zur Umrechnung von Vfm in Efm vorgeschlagen (Elsasser et al., 2020, S. 19; Schluhe et al., 2018, S. 71). Im Rahmen dieser Analyse wurde der Faktor jedoch aus zwei verschiedenen Gründen nicht genutzt: Erstens handelt es sich beim Baumvolumen um ein Erntefestmetermaß, welches ohne Rindenabzüge berechnet wird. Zweitens ging bereits aus Abbildung 18 hervor, dass der Verlauf zwischen dem Baumvolumen und dem Volumen (Vfm) nach Bergel (1973) nicht linear ist. Aus diesen Gründen wird die Umrechnung

\footnotetext{
${ }^{47}$ Der Durchmesser des Kreisflächenmittelstammes wurde durch die Verrechnung der Grundfläche und der Stammzahl im Vorfeld für jede Altersklasse berechnet.
} 
zwischen den beiden Größen in einem eigenen Modell beschrieben (Formel 21). Für die Berechnung des Volumens in Vfm wurde die Schätzfunktion nach Bergel (1973) verwendet. Der Umrechnungsfaktor beträgt beim kleinstem Einzelbaumvolumen 0,95 und sinkt mit steigendem Volumen (Vfm).

$$
f(V o l)=0,95 * V_{o l} l_{V f m}-0,06 * \operatorname{Vol}_{V f m}{ }^{2}
$$

Formel 21: Berechnung des Baumvolumens durch die Nutzung des stehenden Einzelbaumvolumens nach Bergel in Vfm (1973).

Unter den Modellprämissen des Kahlschlags der Fläche und einer gleichmäßigen Verteilung der Bäume innerhalb dieser, gleicht die Entfernung zwischen den Entnahmebäumen dem mittleren Baumabstand. Dieser Abstand entspricht der Wurzel des Quotienten aus der Flächengröße geteilt durch die Stammzahl. Er wird für jeden Baum angenommen und spiegelt die simulierten Abstände gut wider, da das Java Tool sehr homogene Stammverteilungen erzeugt.

Die Qualität der Einzelbäume wird analog zu Kapitel 7.2.2 im Rahmen eines Qualitätsfächers ergänzt. Für jede getestete Qualitätsstufe wird hierbei erneut eine Kopie des Datensatzes erzeugt, in dem alle Werte bis auf die Qualität unverändert bleiben.

Die Vorhersage des Kronentyps erfolgt im Rahmen einer geordneten logistischen Regression. Über das in Formel 22 dargestellte Model lassen sich für jeden Baum drei Wahrscheinlichkeiten (eine für jeden Kronentyp, in Summe $100 \%$ ) berechnen. Im Anschluss daran erfolgt die Ziehung einer Zufallszahl und die Zuordnung zu einem Kronentyp.

$$
\begin{aligned}
& \operatorname{logit}(\hat{P}(Y \leq \text { wipfelschäftig }))=2,78-0,97 * \text { Vol }-0,29 * \text { Qualität } \\
& \operatorname{logit}(\hat{P}(Y \leq \text { zwieselförmig }))=3,51-0,97 * \text { Vol }-0,29 * \text { Qualität }
\end{aligned}
$$

Formel 22: Geordnete logistische Regression des Kronentyps je Baum. $N=181$; $A I C=307$.

Der letzte fehlende Kostentreiber ist die Anzahl an ausgehaltenen Sortimente bzw. die Wahrscheinlichkeit der Sortenanzahl pro Baum. Wie Abbildung 28 zeigt, wird die Aushaltung neben dem Baumvolumen auch von der Qualität des Entnahmebaumes beeinflusst. Je schlechter die Qualität, desto geringer ist die Wahrscheinlichkeit, dass eine zusätzliche Sorte ausgehalten werden kann. Die Vorhersage erfolgt - wie bereits beim Kronentyp - erneut über ein geordnetes logistisches Modell (Formel 23). 
Ergebnisse

$$
\begin{aligned}
& \operatorname{logit}(\hat{P}(Y \leq 1))=1,15-2,03 * \text { Vol }-(-0,25) * \text { Qualität } \\
& \text { logit }(\hat{P}(Y \leq 2))=3,72-2,03 * \text { Vol }-(-0,25) * \text { Qualität } \\
& \text { logit }(\hat{P}(Y \leq 3))=6,72-2,03 * \text { Vol }-(-0,25) * \text { Qualität }
\end{aligned}
$$

Formel 23: Geordnete logistische Regression der Anzahl an ausgehaltenen Sorten je Baum. Dargestellt und berechnet für die ersten vier Sorten. $N=361 ; A I C=579$.

Das Produktivitätsmodell (Formel 20) lässt sich nun auf den Datensatz anwenden. Im nächsten Schritt müssen die Produktivitätswerte zu Kosten pro $\mathrm{m}^{3}$ umgerechnet werden. Um dies zu ermöglichen, müssen allerdings noch die Kostensätze des Holzerntesystems integriert werden. Laut Angaben der Arbeitsgemeinschaft forstlicher Lohnunternehmer (AFL) Niedersachsen e. V. können die Kosten einer Arbeitsstunde eines Harvesters dieser Leistungsklasse mit rund $192 €$ /MAS kalkuliert werden (AFL Niedersachsen e. V., 2017, S. 170). Die letzte verbleibende Variable ist der Korrekturfaktor. Er gibt das Verhältnis zwischen $\mathrm{MAS}_{0}$ und MAS an, also den Anteil an allgemeiner Zeit. In Anlehnung an Pausch (2002, S. 88 u. 107), wird ein Aufschlag von 44,625\% allgemeiner Zeit auf die $\mathrm{MAS}_{0}$ angenommen.

Das Ergebnis dieser beispielhaften Kalkulation ist in Abbildung 38 dargestellt. Es zeigt noch einmal den besonderen Einfluss der Baumqualität auf den Prozess der mechanisierten Buchenaufarbeitung. Zum einen ist der Einfluss auf die Holzerntekosten enorm, zum andern wird auch die Erlösseite beeinflusst. Wie bereits Abbildung 28 zeigte, steigert auch hier eine hohe Qualität die Wahrscheinlichkeit, eine zusätzliche Sorte auszuhalten. Darüber hinaus deckt sich das Ergebnis sehr mit den erwarteten Werten aus der Praxis (AFL Niedersachsen e. V., 2017, S. 177). 


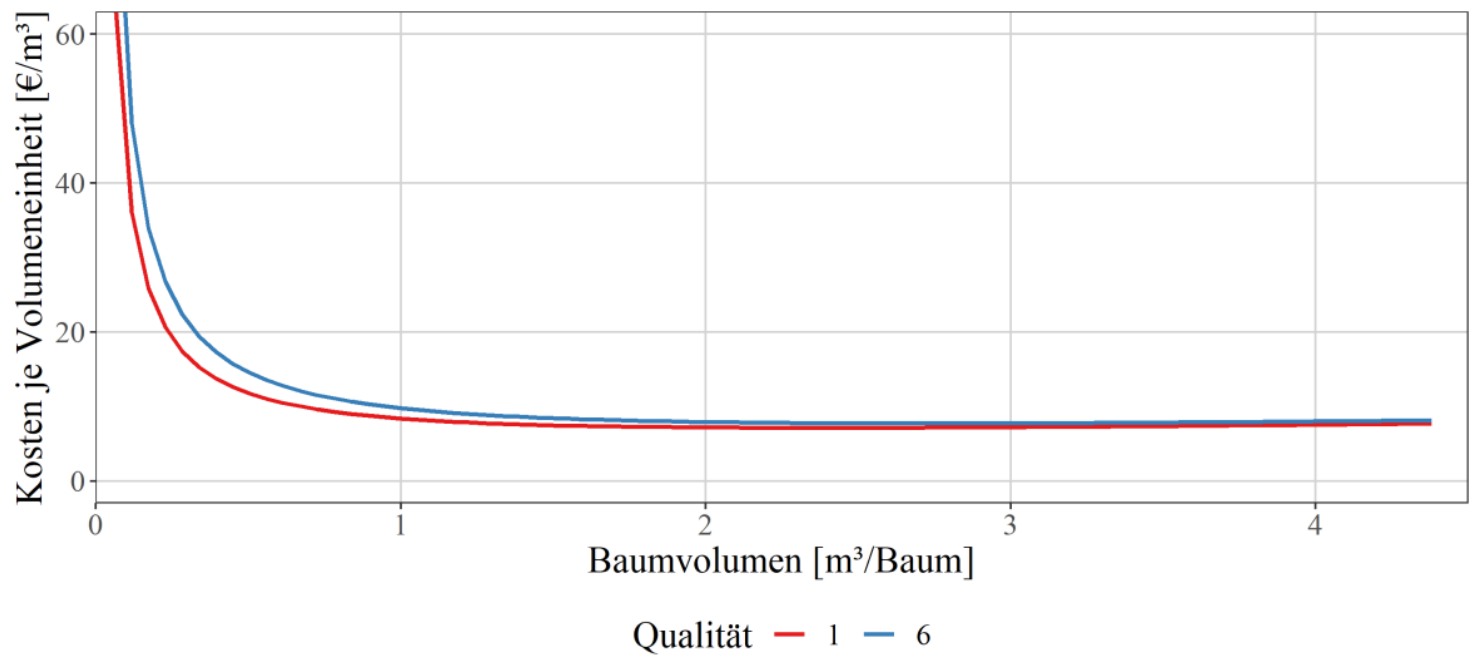

Abbildung 38: Kosten für die Aufarbeitung (Kosten = 192€/MAS) in Abhängigkeit des Baumvolumens. Kalkuliert auf Basis der $\mathrm{MAS}_{0}$ mit einem Aufschlag allgemeiner $\mathrm{AZ}=44,625 \%$. Dargestellt für die Qualitätsklassen 1 und 6.

\subsubsection{Einordung in bestehende Produktivitätsmodelle}

Die Forschungshypothese H5 (Kapitel 5) zielt auf den Vergleich des Produktionsmodells dieser Arbeit mit bestehenden Modellen aus der Literatur ab. Die Einordnung erfolgt rein graphisch durch einen Vergleich der Kurvenverläufe der Modelle in ihren jeweiligen Gültigkeitsbereichen. Alle gezeigten Modelle beschreiben die RAZ, also die MAS 0 ihres untersuchten Harvestersystems. Der erste Vergleich erfolgt - gemäß Hypothese 5a - mit anderen Produktivitätsmodellen der mechanisierte Buchen- bzw. Laubholzaufarbeitung. Wie bereits in Kapitel 4.4.3 aufgezeigt, existieren nur wenige Produktivitätsmodelle für die mechanisiere Buchenaufarbeitung. Als Vergleichsmodelle wurden daher zusätzlich auch Produktivitätsmodelle weiterer Laubholzarten genutzt. Eine Übersicht über die Funktionen und technische Anmerkungen zu den Modellen (eingesetzte Harvestersysteme, Gültigkeitsbereich, etc.) zeigt Anhang 16. Die Modelle von Guglhör (1994, S. 698) und Cremer (2008, S. 72) beinhalten als unabhängige Variable nicht das Baumvolumen, sondern den BHD. Um den Vergleich mit dem Modell dieser Arbeit dennoch zu ermöglichen, wurde der BHD in das Baumvolumen umgerechnet. Mit den Daten des Parametrisierungsdatensatzes wurde ein Polynom zweiten Grades zur Umrechnung geschätzt, welches dann in die Formeln von Guglhör und Cremer eingesetzt wurde (Anhang 17). Die Koeffizienten dieses Polynoms sind $a=46,47$ und $b=-10,55\left(R^{2}=0,95\right)$. 
Alle Modelle wurden des Weiteren zu einem eindimensionalen Modell mit ausschließlich dem Volumen vereinfacht. Hierzu wurden alle Variablen mit Ausnahme des Volumens durch mittlere Ausprägungen ausgerechnet und somit eliminiert. Dies kann unter Umständen einen enormen Einfluss auf den Funktionsverlauf haben (Kapitel 7.2.2). Im Modell von Cremer (2008, S. 72) kann beispielsweise das eingesetzte Harvestersystem über Dummy-Variablen verändert werden. Für die Darstellung wurde das Harvestersystem ausgewählt, welches von der technischen Ausstattung am ähnlichsten zu den Systemen dieser Arbeit war (Anhang 16). Da das Produktivitätsmodell dieser Arbeit deutlich mehr Variablen integriert, wurden im Vergleich (Abbildung 39) zwei Varianten abgebildet, die die Bandbreite der möglichen Ausprägungen abbilden sollen.

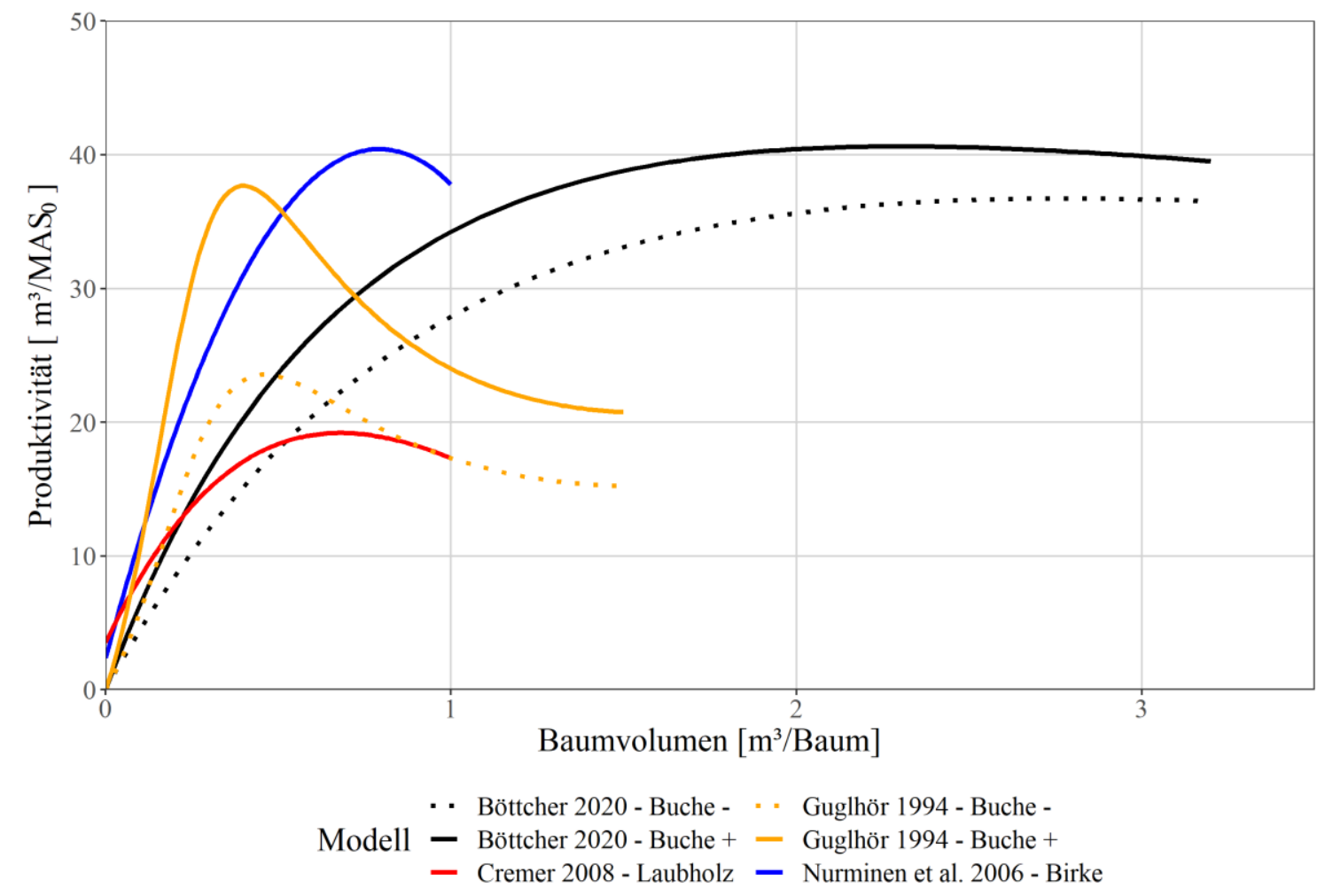

Abbildung 39: Vergleich der Produktivitätsmodelle für die mechanisierte Aufarbeitung von Laubholz. Das Modell „Böttcher 2020 - Buche +“ zeigt die bestmögliche Variablenausprägung (Qualität = 1, Kronentyp = wipfelschäftig); „Böttcher 2020 - Buche -“ zeigt die unproduktivste Variablenausprägung (Qualität $=6$, Kronentyp = besenförmig). In beiden Modellen beträgt die Sortenanzahl $=1$ und die Entfernung $=15 \mathrm{~m}$. 
Es wird deutlich, dass sich das Produktivitätsmodell dieser Arbeit im Bereich bis $0,5 \mathrm{~m}^{3}$ Baumvolumen gut in den Forschungsstand einfügt. Diese Tatsache unterstreicht noch einmal die Vorteile eines Produktivitätsmodells auf Einzelbaumbasis. Denn obwohl sich die Untersuchungsbestände zwischen den jeweiligen Studien in ihren forstlichen Kennzahlen (z. B. Dg) deutlich unterscheiden, so sind die Bestände dennoch zu einem gewissen Teil aus vergleichbaren Einzelbäumen zusammengesetzt. Darüber hinaus zeigt sich die Besonderheit dieser Untersuchung in einem außerordentlichen Gültigkeitsbereich. Mit Ausnahme der Untersuchung von Guglhör (1994) - der darin auch Einzelbäume bis 1,5 $\mathrm{m}^{3}$ berücksichtigte - endet dieser Bereich bei einem Baumvolumen von einem $\mathrm{m}^{3}$. Dieser substanzielle Unterschied zwischen dem bestehenden Forschungsstand und dieser Arbeit hat auch Einfluss auf das optimale Baumvolumen, also den Punkt der maximalen Produktivität. Je nach Modell bewegt sich dieser zwischen 0,4 und 0,8 $\mathrm{m}^{3}$. Mit steigendem Baumvolumen sinkt die Produktivität teils drastisch ab. Im Gegensatz dazu konnte in der vorliegenden Arbeit aufgezeigt werden, dass sich für die untersuchten Harvestersysteme diese Leistungsgrenze erst bei 2,1 bis 2,4 $\mathrm{m}^{3}$ einstellt (in Abhängigkeit von den anderen Modellvariablen). Das eingesetzte Harvestersystem und die technischen Entwicklungen der letzten Jahre scheinen somit einen erheblichen Einfluss auf den Verlauf der Produktivitätsmodelle für die mechanisierte Aufarbeitung von Laubholz zu haben. Die Forschungshypothese 5a kann daher nur bedingt bestätigt werden.

Nachdem eine Einordnung in die bestehenden Modelle der mechanisierten Buchen- und Laubholzaufarbeitung stattgefunden hat, richtet sich der Fokus nun auf den Vergleich mit Modellen auf der Basis von Nadelhölzern und somit auf die Überprüfung der Hypothese 5b. Aus der Vielzahl an Forschungsarbeiten, die sich mit der mechanisierten Aufarbeitung in nadelholzdominierten Wäldern beschäftigen, wurden die Modelle ausgewählt, denen ein ähnlich breiter Gültigkeitsbereich zugrunde liegt. Eine Ausnahme bildet das Fichtenmodell von Cremer (2008, S. 72), welches die Vergleichsmöglichkeiten im unteren Volumenbereich erhöhen soll. Eine Übersicht über die Funktionen und technische Anmerkungen (eingesetzte Harvestersysteme, etc.) der in Abbildung 40 präsentierten Modelle zeigt Anhang 18. 


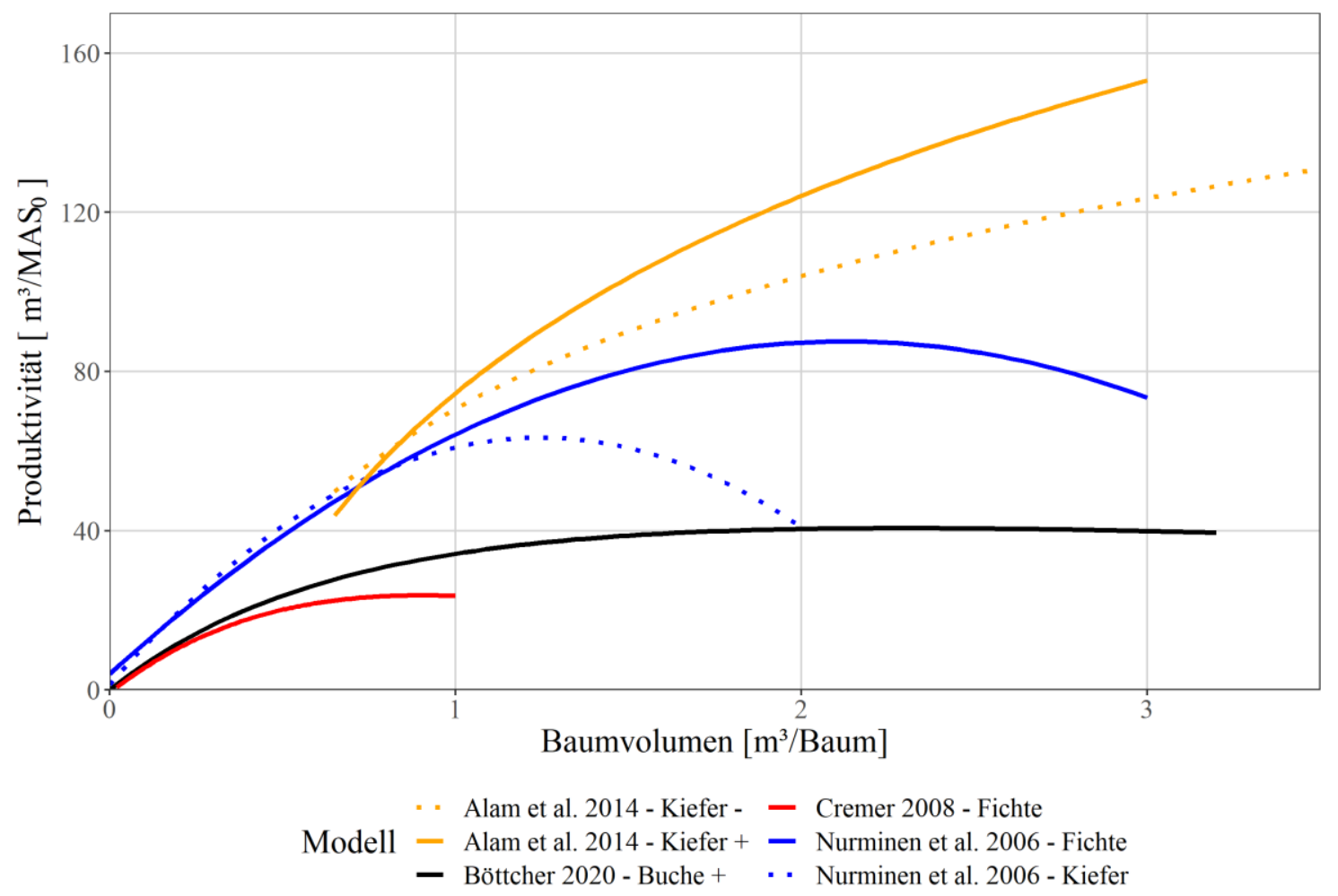

Abbildung 40: Vergleich der Produktivitätsmodelle für die mechanisierte Aufarbeitung von Nadelholz mit dem Modell dieser Arbeit (Formel 20). Das Modell „Böttcher 2020 Buche +“" zeigt die bestmögliche Variablenausprägung (Qualität $=1$, Kronentyp $=$ wipfelschäftig, Sortenanzahl = 1, Entfernung = $15 \mathrm{~m}$ ).

Aus Abbildung 40 geht hervor, dass sich die Leistung der hochmechanisierten Erntemaschinen in Buchenbeständen bis zu einem Baumvolumen von rund 0,4 $\mathrm{m}^{3}$ auf einem ähnlichen Niveau wie in Nadelholzbeständen bewegt, was die Beobachtung von Pausch (2002, S. 122) bestätigt. Diese Aussage gilt aber nur für die Aufarbeitung unter der Annahme der bestmöglichen Variablenausprägungen. Bei einer Aufarbeitung von Buchen mit einer schlechteren Stamm- und Kronenqualität sinkt die Leistung bereits erheblich (Kapitel 7.2.2). Aber auch unter optimalen Bedingungen sinkt spätestens ab einem Baumvolumen von $0,5 \mathrm{~m}^{3}$ die Leistung im Vergleich $\mathrm{zu}$ den Nadelholzmodellen. Die Forschungshypothese H5b kann somit nur tendenziell bestätigt werden.

Eine Ausnahme bildet der Vergleich mit dem Produktivitätsmodell von Cremer (2008, S. 72) für die mechanisierte Aufarbeitung von Fichte, das über seinen kompletten Gültigkeitsbereich eine vergleichbare und später schwächere Produktivität beschreibt. Eine Ursache dafür ist auch hier wieder das eingesetzte Harvestersystem. Ein gutes Vergleichs- 
objekt ist weiterhin das Fichtenmodell von Nurminen et al. (2006, S. 348). Bemerkenswert ist, dass sich der relative Leistungsunterschied mit steigendem Baumvolumen kaum verändert (rund 100\%). Die beiden Funktionsverläufe weisen eine erstaunliche Ähnlichkeit auf und zeigen auch einen vergleichbaren Scheitelpunkt bei einem Baumvolumen von $2,2 \mathrm{~m}^{3}$.

\subsubsection{Summiertes versus direkt geschätztes Produktivitätsmodell}

Die bisher gezeigten Ergebnisse sind auch Folgen des festgelegten Detailgrades dieser Untersuchung. Die Fokussierung auf Aktivitätsebene ist aufgrund der Komplexität des Prozesses auf einem sachlichen Vorgehen begründet (Kapitel 6.2) und ist ein Alleinstellungsmerkmal dieser Arbeit. Wie die Auseinandersetzung mit der bestehenden Studienlage zeigte, wird dieser Detailgrad aufgrund des hohen Arbeitsaufwandes selten angewendet. Für den reinen Vergleich des Produktivitätsmodells mit existierenden Modellen (Kapitel 5, H5) wäre somit ein Prozess-Modell ausreichend gewesen.

Um den Einfluss des hohen Detailgrades zu beleuchten, soll folgend ein Modell gezeigt werden, welches auf dem Gesamtzeitverbrauch für die Aufarbeitung pro Baum beruht und somit direkt geschätzt wird. Dieses Modell folgt also der Annahme, den Prozess der mechanisierten Buchenaufarbeitung als Ganzes zu betrachten und sich nicht auf die jeweiligen Aktivitäten im Detail zu fokussieren. In das Startmodell werden alle verfügbaren Variablen (inklusive der Dummy-codierte Verlaufsvariablen) integriert. Im Anschluss erfolgt die Variablenelimination unter Einbeziehung des AIC-Wertes. Anhang 19 zeigt eine Übersicht über die verbliebenden Regressionsparameter. Die Bewertung erfolgt anhand der Überprüfung zwischen den vorhergesagten und den beobachteten Werten (Abbildung 41). 


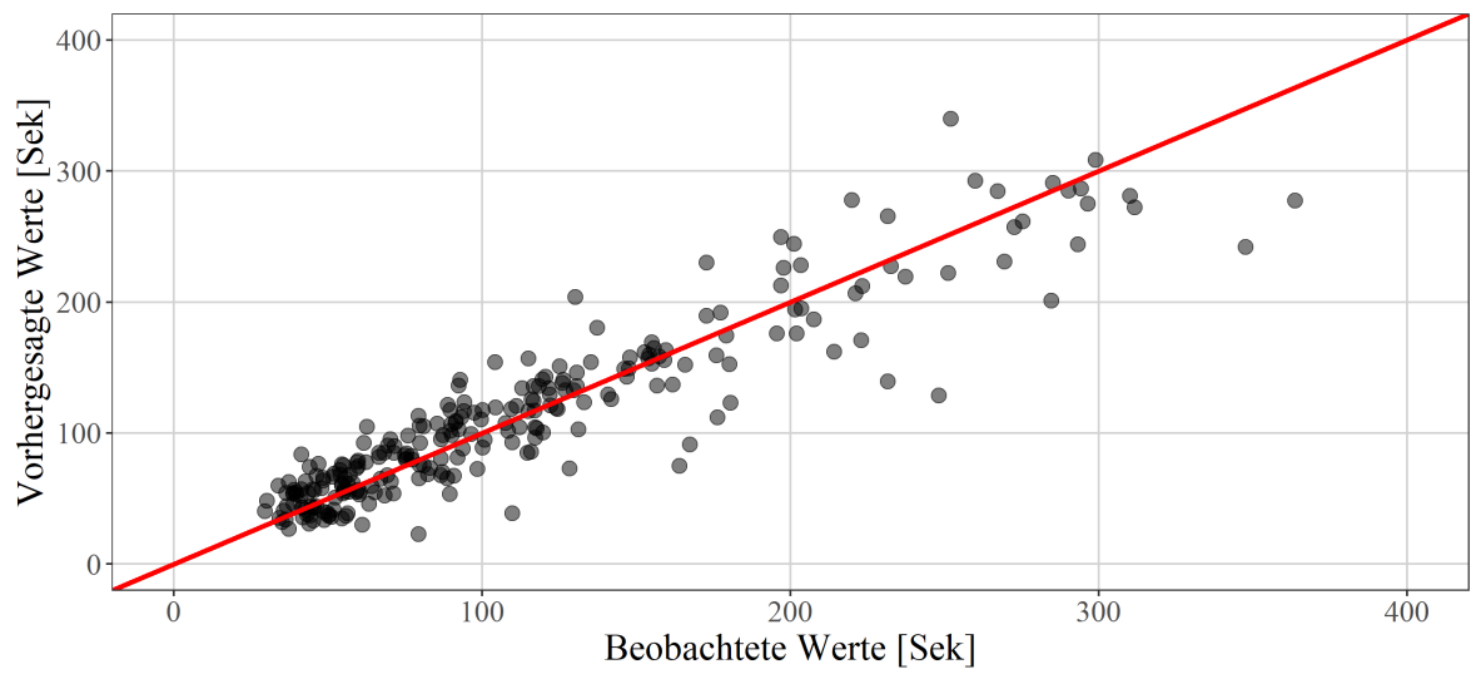

Abbildung 41: Vergleich der beobachteten und der vorhergesagten Werte. Die vorhergesagten Werte wurden durch die Anwendung des direkt geschätzten Modells berechnet (Anhang 19). Die rote Linie zeigt die Winkelhalbierende, bei der die Abweichung $=0$ beträgt. $N=254$.

Das Modell kann die Daten über das ganze Zeitspektrum hinweg sehr gut erklären. Dies bestätigt auch die Residuen-Verteilung zum Modell (Anhang 20), welche normalverteilte Residuen mit einem mittleren Residuum nahe 0 zeigt. Im Vergleich zum kumulierten Gesamtmodell (Formel 18) ist aber eine höhere Varianz erkennbar und der RMSE steigt auf 27,45 Sekunden an. Statistisch gesehen handelt es sich dennoch um ein sehr robustes Modell mit einem $R^{2}=0,86$ und einem $A I C=1.713-$ und das, obwohl eine Vielzahl an Kostentreibern, die auf Ebene der Aktivitäten einen signifikanten Einfluss aufweisen, auf der Prozessebene nicht in das Modell integriert wurden. Dazu zählt beispielsweise die Qualität, welche die Produktivität um bis zu 30 \% beeinflussen kann (Kapitel 7.2.2). Im Gegensatz dazu wurden andere Variablen in ihrer Stärke abweichend eingeschätzt. Um auch hier wieder einen praktischen Nutzen gewährleisten zu können, soll das Modell im folgenden Schritt reduziert werden. Das Vorgehen ist identisch zum kumulierten Modell (Kapitel 7.2.1). Das Ergebnis der Variablenreduktion zeigt Formel 24 mit dem Vergleich der beobachteten und der vorhergesagten Werte in Abbildung 42. 


$$
\begin{aligned}
f(\text { Gesamt }) & =55,86+36,58 * V o l+12,2 * V o l^{2}+0,31 * \text { Entf } \\
& +8,37 * K T_{2}+10,77 * K T_{3}
\end{aligned}
$$

Formel 24: Reduziertes direkt geschätztes Gesamtmodell für die mechanisierte Aufarbeitung von Buche auf der Basis des Modells in Anhang 19. Legende siehe Variablenverzeichnis.

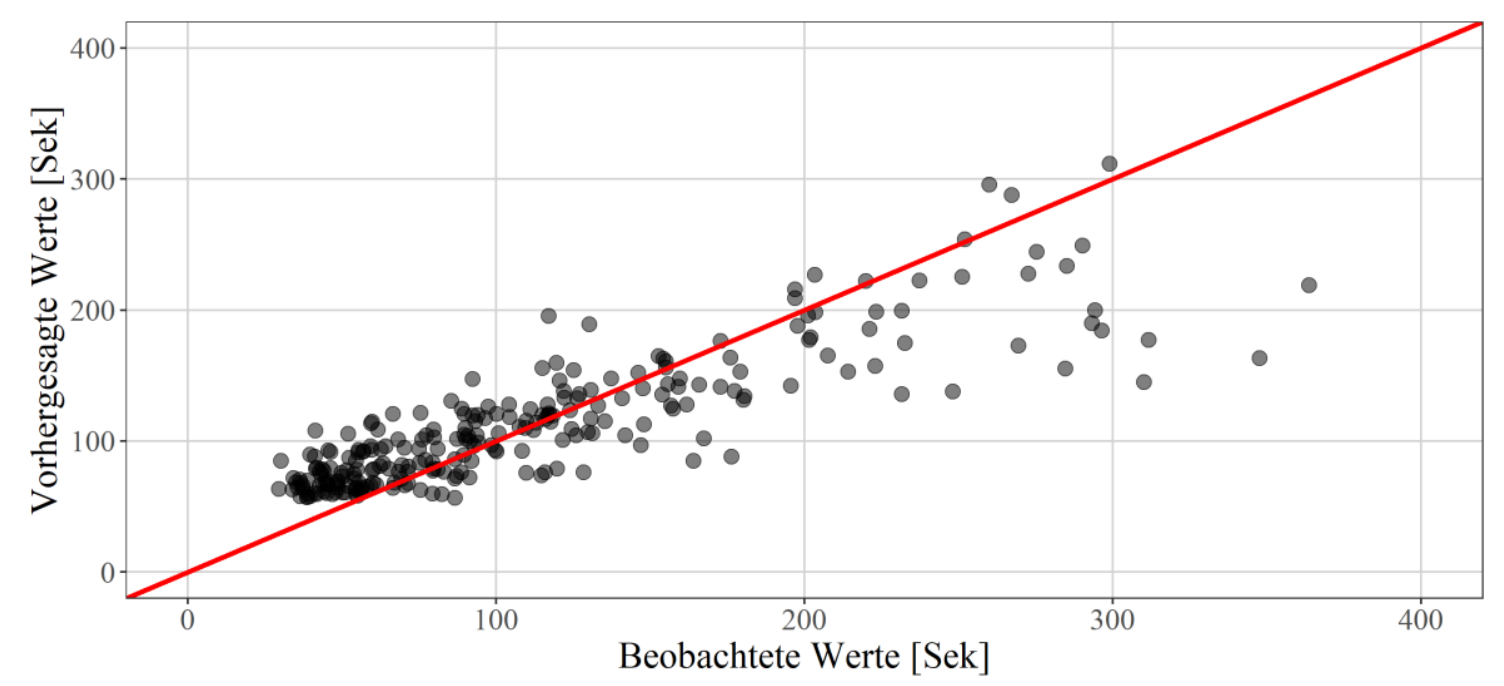

Abbildung 42: Vergleich der beobachteten und der vorhergesagten Werte. Die vorhergesagten Werte wurden durch die Anwendung des reduzierten direkt geschätzten Modells berechnet (Formel 24). Die rote Linie zeigt die Winkelhalbierende, bei der die Abweichung $=0$ beträgt. $N=254$.

Ähnlich wie bereits in Kapitel 7.2.1 beschrieben, zeigt sich auch hier eine Verschiebung der Residuen. Während das Modell im unteren Bereich der beobachteten Werte $(<100$ Sekunden) den Zeitbedarf überschätzt, kommt es bei höheren Werten zu einer Unterschätzung. Der RMSE steigt im Vergleich zum direkt geschätzten Gesamtmodell auf 39,69 Sekunden an. Die Residuen-Verteilung (Anhang 21) ist leicht rechtsschief.

Um eine Aussage über den Einfluss des Detailgrades treffen zu können, ist es nun wichtig, das Modell mit dem reduzierten sachlichen Modell (Formel 19) zu vergleichen. Hierbei werden deutliche Unterschiede ersichtlich. So treten beispielsweise neben dem Baumvolumen nur noch die Entfernung und der Kronentyp als Kostentreiber auf - anders als im gekürzten sachlichen Modell, in welchem ebenfalls die Qualität und die Anzahl an Sorten von zentraler Bedeutung waren. Die Parameter aller Variablen weisen zudem eine andere Stärke auf: Der Einfluss des Baumvolumens und der Entfernung ist im Vergleich 
zum reduzierten sachlichen Modell gesunken, während der Einfluss des Kronentyps deutlich gestiegen ist. Zudem hat sich auch der y-Achsenabstand unverkennbar verändert, was zu einer zusätzlichen Überschätzung des Zeitbedarfes für Bäume mit einem Beobachtungswert kleiner 100 Sekunden führt.

Es lässt sich somit festhalten: Für eine sachgemäße und genaue Einschätzung potentieller Kostentreiber ist es zielführend, den Detailgrad der Untersuchung so hoch wie möglich anzusetzen. Bereits bei dem Wechsel dieser Aggregationsebene (von Aktivität auf Prozess) verschwinden die Effekte zahlreicher relevanter Kostentreiber und die genaue Stärke der verbleibenden Faktoren entspricht nicht mehr den wirklichen Werten. So gehen diese Details beim direkten Anpassen des Produktionsmodells verloren. Dies betont die Wichtigkeit, dass ein analytisches Vorgehen immer eines sachlichen Rahmens bedarf. Es zeigt jedoch auch, dass das direkte Anpassen möglich und ebenfalls valide ist. So sind die Ergebnisse dieser Arbeit direkt mit den Ergebnissen weniger detaillierter Arbeiten aus der Literatur vergleichbar. Mit diesem Ergebnis endet der Ergebnisteil dieser Arbeit. Bevor im nachfolgenden Kapitel die Methodik und das zugrundeliegende Material sowie die Ergebnisse diskutiert werden, erfolgt in Tabelle 19 ein abschließender Gesamtüberblick über alle im Rahmen der Dissertation untersuchten Hypothesen.

Tabelle 19: Übersicht über den Status der aufgestellten Hypothesen (Kapitel 5).

\begin{tabular}{llc}
\hline Hypothese & \multicolumn{1}{c}{ Betrachteter Zusammenhang } & Bestätigt? \\
\hline H1 & Analyse des Prozesses auf Aktivitätsebene & Ja \\
H2 & Grenzproduktivität der mechanisierten Buchenaufarbeitung & Ja \\
H3 & Kronenform und die Qualität des Stammes & Ja \\
& als wichtige Kostentreiber & \\
H4 & Eine steigende Komplexität der Aushaltung vermindert die & Teilweise \\
& Produktivität & \\
H5a & Einordnung in die bestehenden Laubholzstudien & Teilweise \\
H5b & Produktivität der mechanisierten Buchenaufarbeitung ist ge- & Teilweise \\
& ringer als in vergleichbaren Nadelholzstudien & \\
H6 & Detailgrad der Untersuchung hat einen Einfluss auf die Aus- & Ja \\
& wahl und die Stärke der signifikanten Kostentreiber & \\
\hline
\end{tabular}




\section{Diskussion und Fazit}

Kapitel 8 widmet sich der kritischen Auseinandersetzung der Daten, der Methoden und der Ergebnisse dieser Arbeit und ist eingeteilt in vier Unterkapitel. Kapitel 8.1 befasst sich mit der Wertung der Datengrundlage und diskutiert die genutzten Methoden und das zugrundeliegende Material. Im Anschluss daran erfolgt die Diskussion der Ergebnisse in enger Anlehnung an die Forschungshypothesen. Darüber hinaus werden in Kapitel 8.3 Anknüpfungspunkte für zukünftige Forschungsarbeiten thematisiert, bevor letztlich ein übergeordnetes Fazit gezogen wird.

\subsection{Datengrundlage}

\subsubsection{Versuchsflächen und Untersuchungsmaschinen}

Die Auswahl der Versuchsflächen und der geeigneten Harvestersysteme wurde erheblich durch die Starksturmereignisse im Frühjahr 2018 (u. a. Orkantief Frederike) und deren Folgen erschwert. Durch den hohen Anfall von Kalamitätsholz wurde der Frischholzeinschlag im Projektgebiet Südniedersachsen/Nordhessen zwischenzeitlich eingestellt. Aus diesem Grund mussten viele potentielle Versuchsflächen verworfen werden.

Dies ist glückerweise im Hinblick auf das Forschungsdesign dieser Arbeit - mit der Konzentration auf den Einzelbaum als Versuchsobjekt - nicht problematisch. Obwohl nur vier Versuchsflächen aufgenommen werden konnten, wurde eine große Anzahl an Probebäumen erhoben, die eine sehr weite Spanne von Baumattributen abdecken. Durch die sehr fein aufgelöste Untersuchungsmethodik mit dem Einzelbaum oder sogar Einzelbaumbestandteilen (Abschnitten) als kleinstes Untersuchungselement, steht der Einzelbaum im Zentrum der Untersuchung. Daraus folgt, dass die Variabilität zwischen den Bäumen zur Beantwortung der Forschungshypothesen entscheidend ist und nicht die Variabilität zwischen den Versuchsflächen. Einzig standörtliche Unterschiede in den Baumattributen, wie beispielsweise das Höhen-Durchmesser-Verhältnis, konnten nicht analysiert werden. Durch die Konzentration auf die wichtigsten potenziellen Kostentreiber wären ebensolche Variablen allerdings vermutlich ohnehin nicht untersucht worden. Insofern ist eine heterogene Datengrundlage an Einzelbäumen pro Versuchsfläche viel wichtiger als eine hohe Anzahl an Versuchsflächen. Da jeder Baum als unabhängiges 
Versuchselement in die Regressionsanalyse einging, spielt auch die mögliche Verzerrung durch die subjektive Auszeichnung in den Versuchsflächen keine Rolle (Kapitel 4.4.3, Arbeitsaufgabe). Es ist dennoch zweckmäßig, im Vorfeld der Analyse eine systematische Verzerrung zwischen den Versuchsflächen auszuschließen. Um diese zu vermeiden, wurde in jeder Fläche darauf geachtet, ausreichend Einzelbäume zu erheben, um so die Ausprägungsbandbreite der wichtigsten Baumparameter (Kronentyp, Qualität) jeweils über ein breites Einzelbaumvolumen-Spektrum abzudecken. Über eine Varianzanalyse konnte eine Verzerrung zwischen den Versuchsflächen ausgeschlossen werden. Um die Mindestgröße der Stichprobe je Versuchsfläche zu ermitteln, wurde im Rahmen der Voruntersuchung (Kapitel 6.2) der Datensatz solange ausgedünnt (thinning, Iske, 2004, S. 67), bis der Einfluss der signifikanten Kostentreiber für den Gesamtprozess nicht mehr nachweisbar war. Abhängig von der Zielgröße lag die minimale Stichprobengröße bei rund 40 bis 50 Bäumen. Diese Anzahl wurde als Maßgabe für die Aufnahme der weiteren Versuchsflächen genutzt und in jedem Bestand übertroffen. Die Methode des thinnings lässt sich auch zur Bewertung des wechselnden Stichprobenumfanges zwischen den Aktivitätsmodellen nutzen. Je nach Aktivität schwankt die Stichprobengröße zwischen 134 und 361 aufgrund fehlender Variablenausprägungen welche im Vorfeld nicht ausgeglichen wurden. Auch hier zeigte sich in einer Voruntersuchung, dass die Effekte bis zu einer Stichprobengröße von 50 Fällen nachweisbar bleiben.

Eine Problematik, die durch die geringe Anzahl an Versuchsflächen innerhalb der Arbeit verstärkt wurde, ist der Einfluss der Aushaltung auf den Aufarbeitungsprozess. Da nur in einer der untersuchten Versuchsflächen maximal vier Sortimente ausgehalten wurden, fiel die Stichprobenzahl von Bäumen, die in mehr als zwei Sortimente aufgearbeitet wurden, sehr gering aus $(N=44)$. Der Befund, dass die Anzahl der ausgehaltenen Sortimente sich auf die Produktivität der mechanisierten Buchenaufarbeitung auswirkt, hätte mit einer größeren Datengrundlage somit tiefgehender analysiert werden können. Für die Analyse dieser Forschungshypothese wäre ein Ansatz auf der Basis von Bestandesdaten (Kapitel 4.4.1) aufgrund der breiten Differenzierung der Sortimente (z. B. Zopfdurchmesser) besser geeignet gewesen.

Mit der Fokussierung auf private Lohnunternehmer, die in den jeweiligen eigenen Harvestern agierten, wurde die Auswahl an Versuchsflächen zusätzlich erschwert. Die Suche nach geeigneten und bereitwilligen Unternehmern, die adäquate und vergleichbare Bestände aufarbeiteten, bildete dabei eine erhebliche Herausforderung. 
Durch diese Vorgehensweise konnte jedoch sichergestellt werden, dass die Prozessanalyse der mechanisierten Buchenaufarbeitung möglichst praxisnahen Bedingungen unterlag.

Auch die kombinierte Beobachtung von Tätigkeiten eines Menschen und der genutzten Maschine innerhalb eines Arbeitssystems beinhaltet theoretische Probleme (Pausch, 2002, S. 88). Im Rahmen der Untersuchung wurden diese zusammengefasst und nicht getrennt voneinander betrachtet (z. B. keine Unterscheidung zwischen persönlichen oder sachlichen Verteilzeiten). Darüber hinaus gibt es eine erhebliche Anzahl Faktoren, welche die menschliche bzw. maschinelle Leistungsfähigkeit beeinflussen (Kapitel 4.4.3, Arbeitssystem). Aus diesem Grund wurde darauf geachtet, die Eigenschaften des Arbeitsmittels (z. B. Motorleistung, Kranausstattung) und der Maschinenführer (z. B. Erfahrung) so vergleichbar wie möglich zu halten. Da es sich bei den untersuchten Harvestersystemen um erfahrene private Lohnunternehmer in eigenen Harvestern handelte und sehr gute Wetterbedingungen während der Aufarbeitung herrschten, konnte tendenziell davon ausgegangen werden, dass die gemessenen Leistungswerte dem oberen Leistungsbereich zuzuordnen sind (Malinen et al., 2018, S. 110). In der Darstellung der kumulierten Gesamtfunktion (Formel 18) wird dahingegen ersichtlich, dass eine erhebliche Differenz in der Leistung zwischen den Harvestersystemen existiert, die den erwarteten Ergebnissen von Purfürst entspricht (2009, S. 179). Ob diese Unterschiede auf den Fahrer oder die eingesetzte Maschine zurückzuführen sind, oder aber in einer anderen nicht analysierten Störvariable begründet liegen, kann an dieser Stelle nicht bewertet werden.

Wie der Vergleich mit den bestehenden Modellen (Kapitel 7.2.4) zeigte, lässt sich das Produktivitätsmodell dieser Arbeit trotz dieser Limitationen gut in den vorliegenden Forschungsstand einordnen, sodass die bestehende Verzerrung durch die Auswahl besonders produktiver Harvestersysteme als tendenziell gering bewertet werden kann.

\subsubsection{Stehende und liegende Aufnahme}

Wie die Vorstellung des Forschungsstandes in Kapitel 4.4.3 zeigte, ist die Anzahl an leistungsbeeinflussenden Faktoren enorm. Es lassen sich jedoch nicht alle Variablen erheben und nicht alle recherchierten Variablen sind vielversprechend genug, um deren Erhebung im Sinne der forschungsleitenden Fragestellung zu rechtfertigen. Des Weiteren existieren für einzelne Variablen unterschiedliche Skalenniveaus. Aus diesen Gründen ist die Kon- 
zeption der Datenerhebung ein sehr sensibler Punkt. Im Folgenden sollen daher die Aufnahme und die Auswahl ausgewählter Variablen genauer begründet werden.

Einen besonders großen Einfluss auf die mechanisierte Buchenaufarbeitung besitzt die Stamm- und Kronenqualität. Doch hat die Auseinandersetzung mit den bestehenden Studien auch gezeigt, dass es eine Vielzahl an Systemen gibt, um die Stammqualität eines Baumes einzuschätzen (Labelle et al., 2016, S. 178; Suchomel et al., 2012, S. 41). Darüber hinaus kann die Buche unterschiedliche Kronentypen ausbilden, welche in keinem der bestehenden Qualitätssysteme unabhängig von der Stammqualität eingeschätzt werden kann (Kapitel 4.1). Aus diesen Gründen wurde im Rahmen dieser Arbeit nach einer Qualitätseinteilung gesucht, die es erlaubt, die Stamm- und Kronenqualität unabhängig voneinander einzuschätzen. Die Wahl fiel auf ein Verfahren der forstlichen Versuchsund Forschungsanstalt (FVA) Baden-Württemberg. Zum einen handelt es sich um ein erprobtes System, welches von der FVA speziell an die Wuchsmerkmale der Baumart Buche angepasst und bereits innerhalb der BWI in Baden-Württemberg genutzt wurde. Zum anderen ist die praktische Anwendung des Systems durch das geläufige Schulnotensystem und die Zuordnung über Schaubilder selbst für neue Nutzer sehr einfach. In Kombination mit der Kronentypisierung von Hengst (1964, S. 82), die innerhalb dieser Arbeit nach der Beschreibung von Kleinschmit (2015, S. 13, Vernachlässigung des BuckettKronentyps) genutzt wurde, konnte somit eine umfassende Qualitätseinschätzung der Entnahmebäume durchgeführt werden.

Eine Beeinflussung der Maschinenführer hinsichtlich ihrer Orientierung im Wald scheint durch die zusätzliche Kennzeichnung der Bäume (Nummerierung) entstanden zu sein. Alle Maschinenführer gaben auf Nachfrage an, sich nach wenigen aufgearbeiteten Bäumen größtenteils anhand der Nummern orientiert und weniger auf die Auszeichnung des Revierleiters geachtet zu haben, da die Nummern besser sichtbar waren. Dies stellt eine gewisse Abweichung zum Normalfall dar, da voraussichtlich weniger Zeit für die Orientierung in der Fläche benötigt wurde. Es handelt sich hierbei somit um eine mögliche Erklärung, weshalb kein signifikanter Unterschied zwischen den Harvestersystemen für die Aktivität Anfahrt und Positionierung festgestellt werden konnte, da hier für alle Fahrer gleichermaßen bessere Bedingungen geschaffen wurden.

Wie in Kapitel 4.4.3 gezeigt wurde, hat zudem auch die Hangneigung einen signifikanten Einfluss auf die Harvester-Produktivität. Nach Pausch (2002, S. 93) treten für Radharvester mit Bogiebänder-Ausstattung bis zu einer Hangneigung von $30 \%$ keine Produktivitätseinbußen auf. Obwohl keine der untersuchten Maschinen während der Aufarbeitung 
mit Bogiebändern ausgestattet war, wurde die Hangneigung im Zuge der Erhebung nicht an jedem Baum vermessen und konnte folglich auch nicht in die Analyse einbezogen werden. Da sich die Versuchsflächen jedoch generell durch eine geringe Hangneigung zwischen 5 und $15 \%$ auszeichneten, ist nicht davon auszugehen, dass dieses Vorgehen einen Einfluss auf die Aussagekraft der Befunde besitzt. Für eine tiefergehende Analyse der Hangneigung als Bestandesattribut wäre die Bestandesheterogenität ohnehin zu gering gewesen. Da in dieser Arbeit sehr großer Wert auf eine große Baumheterogenität innerhalb der Bestände gelegt wurde, könnten Bestandesattribute wie die Hangneigung in Folgeanalysen mit vergleichsweise geringem Aufwand analysiert werden. Bei einer Anschlussanalyse sollte daher eine große Bestandesheterogenität angestrebt werden. Jedoch kann die Baumheterogenität in den Beständen und der damit verbundene Messaufwand geringer ausfallen.

Ähnliches gilt für den Unterwuchs in den Versuchsflächen. Der Einfluss des Unterwuchses konnte zwar in bestehenden Studien nachgewiesen werden, war in dieser Arbeit jedoch nicht versuchsgegenständlich. Gunnarsson und Hellström (1992, S. 68, in Pausch, 2002, S.123) geben an, dass eine Beeinträchtigung der Harvester-Produktivität ab einer Höhe von 1,3 m entsteht - eine niedrigere Verjüngung behindert den Harvester nicht nennenswert. Da in keiner Untersuchungsfläche die Verjüngung im Mittel über 1,3 m Höhe lag, wurde die Höhe des Unterwuchses am Einzelbaum nicht erhoben.

Die Distanzmessungen erfolgten im Vorfeld der jeweiligen Aufarbeitungsmaßnahme mittels Forestor-Vertex IV der Firma Haglöf. In einer Vorstudie wurde der Einsatz eines GPS-Gerätes (GPSMAP 64x der Firma Garmin) für die Messung der Anfahrtsstrecken getestet - aufgrund der enormen Ungenauigkeit wurde diese Methode jedoch verworfen. Die Messung der Entfernung zum nächsten Baum erfolgte entlang der Rückegasse auf Höhe der beiden Bäume (Kapitel 6.3.1, Messung des Rückegassenabstandes). Da sich der dadurch festgelegte Anfahrtsweg nicht immer mit dem reell zurückgelegten Weg deckte, wurde dieser Teil der Stichprobe von der Analyse der Anfahrt und Positionierung ausgeschlossen. Dieses Vorgehen erklärt die geringere Datensatzgröße für die Anfahrt und Positionierung im Vergleich zu den anderen Aktivitäten (Kapitel 7.1.1). Ähnliches trifft auch auf die Messung der Entfernung zur Rückegasse zu. Auch hier entsprach die theoretisch gemessene Distanz nicht in allen Fällen der reellen Position des Harvesters, weshalb diese Fälle von der Regression ausgeschlossen wurden. Dieses Vorgehen senkte zwar die Stichprobengröße, war aber notwendig, um eine Datenverzerrung auszuschlieBen. 
Die Aufnahme des liegenden Holzes umfasste nur wenige Parameter, welche hauptsächlich durch die RVR vorgegeben waren. Es konnten hier jedoch drei Diskussionspunkte ausgemacht werden, die folgend beschrieben werden.

Der erste Punkt beschäftigt sich mit der Nutzung des Harvesterprotokolls als Datengrundlage für die Abmaße der Abschnitte: Wie die Studienergebnisse einer Bachelorarbeit an der Abteilung Forstökonomie der Georg-August-Universität Göttingen zeigen konnten, ist das Harvesterprotokoll in stärker dimensionierten Buchenbeständen stark fehlerbelastet (Kleinschmit, 2015, S. 48; Neugebauer, 2013). Die Gründe dafür liegen beispielsweise in einer ungenauen Längenmessung durch bögige Äste. Darüber hinaus wurden während der Maßnahme nicht nur die ausgezeichneten Bäume gefällt und aufgearbeitet, sondern auch störende oder beschädigte Bäume. Um das Protokoll nutzen zu können, müssten diese Abschnitte per Hand aus der Protokolldatei aussortiert werden. Daher wurde darauf verzichtet, dass Harvesterprotokoll in den Versuchsaufbau zu integrieren.

Der zweite Diskussionspunkt bezieht sich auf die Berechnung des Baumvolumens, welches auf der Aufnahme der liegenden Abschnitte beruht. Wie bereits in Kapitel 6.3.2 angesprochen, existieren im forstlichen Kontext unterschiedliche Angaben für das Volumen eines Baumes. Innerhalb der Arbeit wurde sich dafür entschieden, die Angabe Baumvolumen $\left(\mathrm{m}^{3}\right)$ zu nutzen, welches als Erntefestmetermaß zuzüglich der Rindenverluste definiert werden kann (Efm m. R.). Diese Entscheidung beruhte auf mehreren Gründen: Erstens beschreibt diese Maßeinheit die tatsächlich manipulierte Holzmenge am geeignetsten. Obwohl speziell für den Holzverkauf die Volumenangabe ohne Rinde wichtig ist, war es für die Überprüfung der forschungsleitenden Hypothesen von großer Relevanz, die bereitgestellten Information so wenig wie möglich zu reduzieren. Für den Harvester ist es unbedeutend, zu welchen Anteilen das manipulierte Volumen aus Rinde oder Holz besteht. Zweitens war ein genauer Abzug der Rindenmenge nicht möglich. Für einen Rindenabzug werden entweder Funktionen wie beispielsweise von Altherr et al. (1974) oder die Angaben der RVR genutzt. Wie die Arbeit von Willems (2015, S. 27) zeigen konnte, führt die Annahme der RVR-Rindenabzüge für die Buche aber zu unnatürlichen Sprüngen im Verhältnis zwischen den Holz- und Rindenanteilen in Abhängigkeit zum Durchmesser. Auch die Annahme eines festen Rindenanteils würde zu einer Verzerrung der Ergebnisse führen, wie der Vergleich in Abbildung 18 gezeigt hat. Letztlich ermöglichte die Verwendung des definierten Baumvolumens eine Vergleichbarkeit mit anderen Modellen. Speziell in englischsprachigen Untersuchungen wird das Baumvolumen in Efm m. R. als Maßeinheit für Produktivitätsmodelle der mechanisierten Aufarbeitung 
verwendet (processed tree volume over bark; u. a. Alam et al., 2014, S. 16; Labelle et al., 2016, S. 178). Wie die vorgestellten Studien in Kapitel 7.2.4 zeigen, wird diese Angabe jedoch vor allem im deutschsprachigen Raum nicht immer verwendet. Hier wird stattdessen mitunter der BHD (u. a. Guglhör, 1994, S. 698), das Vorratsvolumen (u. a. Heide et al., 2005, S. 963) oder das Erntevolumen ohne Rinde (u. a. Purfürst, 2009, S. 102) als unabhängige Variable verwendet. Da jedoch eine Entscheidung getroffen werden musste und die Mehrheit der Studien Efm m. R. nutzten, wurde sich gegen dieses Vorgehen entschieden.

Der letzte diskussionswürdige Punkt der liegenden Aufnahme ist die Notwendigkeit des Datenausgleiches (Kapitel 6.3.4). Durch die Verwendung von Spline-Regressionsmodellen wurde versucht, die exakte durchschnittliche Ausprägung der fehlenden Variablen zu finden und auf Grundlage der vollständigen Daten einzusetzen. Splines sind für diesen Zweck bestens geeignet, da sie sich der bestehenden Datenlage möglichst nahe anpassen. Sie kommen ganz ohne (in diesem Zusammenhang verzerrende) Annahmen des Datenverlaufs aus. Die Verwendung solcher Spline-Regressionen hätte jedoch vermieden werden können, wenn die Videosichtung vor der liegenden Aufnahme der Abschnitte erfolgt wäre. Aufgrund des enormen Zeitaufwandes der Videoanalyse war jedoch eine Sichtung des Videos zwischen der Aufarbeitung und vor der Bringung des Holzes nicht möglich. Hinzu kamen organisatorische Probleme in der Versuchsfläche Hessen 2 (Tabelle 4): Ein Teil der liegenden Abschnitte wurde gerückt, bevor diese vom Aufnahmetrupp vermessen werden konnten. Da der relative Anteil der ausgeglichenen Daten aber als gering einzustufen ist und die Methodik sorgfältig ausgewählt wurde, sollte kein nennenswerter Bias durch die Ausgleichsfunktionen in die Datengrundlage eingegangen sein. Eine visuelle Untersuchung aller Verteilungen der aufgefüllten Variablen bestätigt diese Annahme. Die Füllung der Datenlücken war demnach vorteilhaft, da so für alle Regressionsanalysen die größtmögliche Anzahl an Beobachtungen zur Verfügung stand, ohne den Datensatz zu verzerren.

\subsubsection{Zeitstudie}

Wie auch in anderen Studien beschrieben (Kleinschmit, 2015, S. 105; Purfürst, 2009, S. 7), hat sich ebenfalls für das Forschungsinteresse dieser Arbeit die Durchführung einer videobasierten Zeitstudie als besonders geeignet angeboten. Es ist jedoch anzumerken, dass das Filmen eines Probanden zu einem selbstauferlegten Leistungsdruck und somit 
zu einer Beeinflussung seines Verhaltens führen kann. Dieses psychologische Phänomen ist auch als soziale Erwünschtheit bekannt (Hossiep, 2019, 1. Absatz): Der Proband agiert in Folge der Untersuchung so, wie es seiner Ansicht nach von ihm erwartet wird. Nach Purfürst (2009, S. 172) wird sich der Maschinenführer des Harvesters, im Wissen der Untersuchung, möglichst produktiv verhalten. Durch eine indirekte Dokumentation (Kamera nicht im Sichtbereich, tagesweise Aufnahmen, etc.) sollte diesem Sachverhalt entgegengewirkt werden. Zudem konnte durch die Aufnahme eines ganzen Arbeitstages die menschliche Leistungsdisposition im Tagesverlauf berücksichtigt werden, was ein weiterer großer Vorteil des gewählten Verfahrens ist (Pausch, 2002, S. 88).

Eine andere Möglichkeit der Erhebung hätte die Durchführung einer sensorgestützten Zeitstudie geboten. Diese basiert auf einer Messung festgelegter Bewegungen eines Harvester mithilfe verschiedener Sensoren (Purfürst, 2009, S. 56-60). Sensorgestützte Zeitanalysen sind jedoch besonders störanfällig und zeichnen sich durch eine hohe Installationszeit aus. Da es sich bei den untersuchten Harvestersystemen um private Dienstleister handelte, sollte deren aufgebrachte Zeit so gering wie möglich gehalten werden. Darüber hinaus sollte die Untersuchung eher unbewusst für die Maschinenführer ablaufen. Aus diesen Gründen wurde sich gegen dieses Verfahren entschieden.

Aus dieser Entscheidung haben sich jedoch nicht nur Vorteile, sondern auch ein zentraler Nachteil ergeben, der sich in der benötigten Zeit für die Analyse widerspiegelte. So haben sich die manuelle Analyse des Videomaterials sowie die Messung und Zuordnung der Aktivitäten als besonders zeitintensiv herausgestellt. Für die Codierung von einer Stunde Videomaterial wurden rund sechs bis acht Stunden beansprucht. Durch den Einsatz mehrere Codierer könnte hierbei ein Bias entstehen (Nuutinen et al., 2008, S. 70): Je erfahrener dieser ist, desto geringer ist sein mittlerer Messfehler bei der Messung der Ablaufabschnitte. Da in der vorliegenden Arbeit die Materialien allerdings von lediglich einem Codierer (dem Autor) analysiert wurden, ist in diesem Fall von einer hohen Intra-CoderReliabilität auszugehen.

Letztlich soll an dieser Stelle ein Blick auf den Untersuchungsschwerpunkt der reinen Arbeitszeit gerichtet werden. Jede Einbeziehung auftretender allgemeiner Zeiten sorgt in dieser Definition für Verzerrungen. Aus diesem Grund wurde in der Untersuchung die MAS $_{0}$ als Untersuchungsgröße gewählt, was sich mit dem bestehenden Forschungsstand für die Erstellung von Produktivitätsmodellen auf der Basis von Einzelbaumwerten deckt (u. a. Cremer, 2008, S. 72; Mederski et al., 2016, S. 30; Nurminen et al., 2006, S. 347). Im Gegensatz dazu werden in Untersuchungen, die auf der Basis von Bestandesdaten 
erstellt wurden, häufig die Aufschriebe der Maschinenlaufzeit genutzt, welche sich auf die MAS $_{15}$ beziehen (Purfürst, 2009, S. 16). Der zusätzliche Zeitbedarf, der zwischen diesen beiden Größen liegt und als Korrekturfaktor bezeichnet wird, wurde bereits in vielen Studien thematisiert (u. a. Ovaskainen et al., 2004, S. 74; Sirén \& Aaltio, 2003, S. 41; Spinelli et al., 2009, S. 43). Pausch (2002, S. 92) geht beispielsweise von einem Anteil von $20 \%$ an allgemeiner Zeit innerhalb der $\mathrm{MAS}_{15}$ aus. Purfürst (2009, S. 173) fasst in seiner Arbeit die bestehenden Forschungsstand zum Thema Korrekturfaktoren zwischen Zeitstudien und der Auswertung von Betriebsbelegdaten zusammen und hält zwischen der $\mathrm{MAS}_{15}$ und der $\mathrm{MAS}_{0}$ ein Verhältnis von $1 \mathrm{zu}$ 1,5 für angemessen. Dieser Faktor beinhaltet neben der Einbeziehung der allgemeinen Zeiten unter 15 Minuten auch den Einfluss eines eventuellen Leistungsdruckes durch die Durchführung einer Zeitstudie oder schwankende Umweltbedingungen. Wie die Ergebnisse in Tabelle 4 für diese Arbeit zeigen, beträgt der Anteil der allgemeinen Zeit in der gemessenen $\mathrm{MAS}_{15}$ zwischen 7 und $23 \%$, in Abhängigkeit zum eingesetzten Harvestersystem.

Darüber hinaus beschreibt die $\mathrm{MAS}_{15}$ noch nicht die Gesamtarbeitszeit, da sie sachliche und persönliche Verteilzeiten von über 15 Minuten Länge exkludiert. Auch für diese Verrechnung existieren Korrekturfaktoren. Spinelli et al. (2009, S. 43) weisen beispielsweise einen zusätzlichen Zeitbedarf von 20,9\% für die allgemeinen Zeiten über 15 Minuten bezogen auf die $\mathrm{MAS}_{0}$ aus. Nach Pausch (2002, S. 107) beträgt der Aufschlag für Radharvester $15,7 \%$, bezogen auf die $\mathrm{MAS}_{15}$. Diese Faktoren hängen jedoch stark von der allgemeinen Arbeitssituation (z. B. Umsetzungen der Maschine) ab. Eine Berechnung eigener Korrekturfaktoren war aufgrund des verhältnismäßig kurzen Untersuchungszeitraumes nicht möglich.

\subsubsection{Datenanalyse}

Die Datenanalyse wurde grundsätzlich durch den theoretischen Rahmen der Arbeit (Kapitel 3.3.1) und durch die Maßgabe, ein praktisch anwendbares Modell zu entwickeln, vorgegeben.

Der theoretische Rahmen bestimmte in erster Linie den funktionalen Zusammenhang des Produktivitätsmodells und die Transformation der abhängigen Variablen. Wie Kapitel 3.3.2 zeigt (Tabelle 1), existieren eine breite Anzahl an möglichen Transformationen des Baumvolumens in der bestehenden Literatur. Folgt man aber den theoretischen Zusammenhängen des Stückvolumengesetzes (Hilf, 1928), wird deutlich, dass es sich bei der 


\section{Diskussion und Fazit}

Produktivitätsfunktion um die Reziproke-Hyperbel der Funktion zur Erklärung des Zeitbedarfes pro Baum handelt - unabhängig von dessen Funktionsart. Dieser Annahme folgend kann der Zeitbedarf pro Baum direkt geschätzt (klassisch linear, oder anderweitig) und im Anschluss in ein Produktivitätsmodell überführt werden.

Der Anspruch, ein Modell zu entwickeln, welches relativ leicht in der Praxis nutzbar ist, beeinflusst insbesondere die Genauigkeit des Modells (Kapitel 7.2.1). Durch die systematische Kürzung von Variablen wird das Produktivitätsmodell zwar vereinfacht, nimmt jedoch in der Genauigkeit der Schätzung ab. Die Datengrundlage wird durch das gekürzte Modell jedoch immer noch zufriedenstellend erklärt (Abbildung 35).

Ein abschließender Blick soll auf das Akaike-Informationskriterium (AIC) gerichtet werden, welches als Maßgabe der Güte eines Modells genutzt wurde. Diesem statistischen Wert kommt innerhalb der Arbeit eine zentrale Rolle zu. So entschied die $A I C$-gesteuerte rückwärtsgerichtete Variablenelimination innerhalb der Aktivitätsanalyse, welche Variablen in die abschließenden Modelle integriert wurden. Die Vorteile dieser Methodik sind nicht nur in der Theorie beschrieben (Kapitel 6.3.4), sondern zeigen sich auch praktisch am Beispiel der Aktivität Entasten. Ohne eine AIC-gesteuerte Variablenelimination wäre beispielweise die Variable „Anzahl Sorten“ aufgrund ihrer nicht signifikanten Stärke im Maximalmodell (Anhang 7) nicht in das abschließende Modell der Aktivität integriert worden - obwohl diese Variable einen wichtigen Einfluss auf die Gesamtproduktivität aufweist (Kapitel 7.2.2).

\subsection{Forschungshypothesen}

Auf Basis der präsentierten Ergebnisse und unter Berücksichtigung des Forschungsstandes können in Bezug auf die forschungsleitenden Hypothesen dieser Arbeit die folgenden Antworttendenzen abgeleitet werden.

H1: Der Prozess der mechanisierten Buchenaufarbeitung lässt sich in verschiedene Aktivitäten zerlegen, wobei jede dieser Aktivitäten mit einem separaten Produktivitätsmodell beschrieben werden kann. 
Wie die Ergebnisse der Voruntersuchung zeigten, lässt sich der Prozess der mechanisierten Buchenaufarbeitung tatsächlich in Form eines Tätigkeitsmodells beschreiben (Abbildung 15, Tabelle 6). Das Grundmodell orientiert sich hierbei an der Arbeit von Alam et al. (2014, S. 17), welches an die Aktivitäten der mechanisierten Buchenaufarbeitung angepasst wurde.

Als eine Besonderheit wurden im Rahmen dieser Dissertation Zusatzaktivitäten integriert, die als gestörte Ausführung einer definierten Aktivität verstanden werden können. Diese wurden zwar bereits in anderen Studien bemerkt (Kapitel 6.2), aber noch nicht statistisch ausgewertet. Zusammenfassend lässt sich durch die Erstellung eines aktivitätsabhängigen Prozessmodells der erste Teil der Hypothese bestätigen. Für die Überprüfung des zweiten Teils erfolgt nachfolgend eine kurze Diskussion der separaten Aktivitätsmodelle:

Die Aktivität der Anfahrt und Positionierung beinhaltet eigentlich zwei getrennte Aktivitäten, die jedoch aufgrund einer zu starken Überlappung anhand des Videomaterials nicht objektiv voneinander getrennt werden können (Kapitel 6.2). Dennoch liegt die Vermutung nahe, dass der Einfluss der zurückgelegten Entfernung vorrangig die Anfahrt erklärt, während der fixe Zeitanteil der Aktivität auf die Positionierung zurückzuführen ist. Folgt man dieser Annahme, so lässt sich anhand der Variablenausprägung eine mittlere Anfahrtsgeschwindigkeit berechnen, die mit dem bestehenden Forschungsstand verglichen werden kann. Pausch (2002, S. 107) weist in seiner Untersuchung eine Anfahrtsgeschwindigkeit von $11 \mathrm{~m} / \mathrm{min}$ aus. Ovaskainen et al. (2004, S. 72) berechnen einen Wert, der in Abhängigkeit des Harvestersystems zwischen 11 und $22 \mathrm{~m} / \mathrm{min}$ liegt. Unabhängig vom eingesetzten Harvestersystem liegt die festgestellte Anfahrtsgeschwindigkeit dieser Arbeit bei $26,4 \mathrm{~m} / \mathrm{min}$. Dies scheint plausibel, betrachtet man die guten Bedingungen während der Aufarbeitung (keine Nässe, schwache Hangneigung). Der berechnete Zeitbedarf für die Positionierung erscheint mit 22 Sekunden jedoch tendenziell zu hoch (vgl. Ovaskainen et al., 2004, S. 73). Daraus lässt sich ableiten, dass die Aktivität in der Zeitstudie nicht weiter aufgeschlüsselt werden konnte, sich im Rahmen der Regressionsanalyse jedoch realistische Tendenzen ableiten lassen. Dieses Ergebnis könnte in zukünftigen Untersuchungen noch separiert und validiert werden.

Der Zeitverbrauch des Greifens eines Baums zeigt nur einen geringen Volumentrend. Die Steigung ist so gering, dass dieser Zusammenhang auch als fixer Zeitbedarf interpretiert werden kann. Da das Greifen eine vorbereitende Aktivität für die Fällung darstellt, 
könnte der schwach vorhandene Volumentrend in einem Zusammenhang mit den Überlegungen des Maschinenführers bezüglich der Fällung stehen (z. B. Entscheidung über die Fällrichtung). Ein unerwartetes Ergebnis stellt der nicht signifikante Einfluss der Entfernung des Baumes zur Gasse auf die benötigte Zeit des Greifens dar. Dieser Befund kann zum einen in einer Ungenauigkeit innerhalb des methodischen Konzepts der Entfernungsmessung begründet liegen (Kapitel 8.1.2). Zum anderen wurde dieser fehlende Zusammenhang bereits in einer Studie von Ovaskainen et al. (2004, S. 73) bemerkt. Zwar wurde innerhalb dieser Untersuchung das Greifen zur Fällung gezählt, es zeigte sich aber auch hier kein signifikanter Zusammenhang zwischen der Entfernung zum Baum und dem benötigten Zeitbedarf (Anhang 6). Es kann daher geschlussfolgert werden, dass der Effekt der Entfernung des Baumes zur Gasse nicht relevant ist, solange sich der Baum innerhalb des Aktionsradius des Harvester befindet.

In der Analyse der Fällung konnte ein überproportionaler Zeitbedarf in Abhängigkeit zum Baumvolumen nachgewiesen werden, was auf Basis bestehender Modelle nicht erwartet wurde (u. a. Nurminen et al., 2006, S. 344). Dies ist vermutlich in den morphologischen Unterschieden zwischen den Baumarten begründet, denn die Fällung einer Buche wird mit steigendem Baumvolumen überproportional komplexer: Nicht nur steigt durch die größer werdende Kronenauslage die Schwierigkeit einer bestandespfleglichen Fällung, sondern der Baum muss zudem aufgrund seines Gewichtes ab einem bestimmten Volumen während der Fällung losgelassen werden (Kapitel 7.1.3). In Folge dessen können weitere Zusatzaktivitäten notwendig werden, um das Entasten beginnen zu können (z. B. Säuberungsschnitt). Weitere Untersuchungen könnten hier ansetzten, um empirisch $\mathrm{zu}$ überprüfen, welche Eigenschaften und Zusammenhänge diesen überproportionalen Zeitbedarf beeinflussen. Die Erkenntnisse zeigen, dass das klassische (lineare) Stückvolumengesetz nicht ausreicht, um den Zeitverbrauch dieser Aktivitäten in Abhängigkeit zum Baumvolumen zu erklären.

Der Zeitbedarf für das Entasten wies ebenfalls einen überproportionaler Zeitbedarf in Abhängigkeit zum Baumvolumen auf. Darüber hinaus besteht eine Besonderheit dieser Aktivitätsmodellierung in der vorausgehenden Analyse des Zeitbedarfes auf Abschnittsebene sowie einer notwendigen Variablentransformation (Kapitel 7.1.4). Zum einen können aus dieser Voranalyse wichtige Erkenntnis über den Einfluss der Aushaltung auf die Entastungsdauer gewonnen werden. Zum anderen ermöglicht sie Aussagen über auftretende Zusatzaktivitäten. Beispielsweise bestätigt der aufgezeigte entstehende Mehraufwand für das Ziehen eines Abschnittes (Vorrücken) während des Entastens ein Ergebnis 
von Väätäinen et al. (2004). Ein überraschender Befund zeigte sich hingegen im signifikanten Einfluss des Harvestersystems. Eine mögliche Erklärung für die Differenzen der Harvestersysteme (Tabelle 13) liefern die unterschiedlichen Vorschubgeschwindigkeiten der Aggregate (Tabelle 5). Die Differenzen zeigten sich aber nur bei der abschnittsweisen Analyse - im Baummodell für das Entasten stellte das Harvestersystem keinen relevanten Kostentreiber mehr dar. Die Reduktion (z. B. Abschnittsherkunft) sowie Transformation von Variablen (z. B. Sortenausprägung am Abschnitt zu Anzahl Sorten am Baum) geht dabei stets mit einem Informationsverlust einher, der zu einer gewissen Verzerrung bezüglich der Schätzung des Zeitbedarfes auf Baumebene beigetragen haben könnte. Diese Verzerrung kann aufgrund der hohen Güte des Modells (Tabelle 14) aber als tendenziell gering eingeschätzt werden.

Die Analyse des Umgreifens bildete einen gesonderten Fall innerhalb der Aktivitätsmodellierung. So musste nicht nur der Zeitbedarf geschätzt werden, sondern auch, ob und wie oft diese Aktivität innerhalb der Aufarbeitung auftrat. Das allgemeine Auftreten sowie die Anzahl der Vorgänge wird vorrangig vom Baumvolumen und dem ausgebildeten Kronentyp bestimmt (Formel 13 \& Formel 14). Der Zeitbedarf für das Umgreifen ist aber nicht direkt vom Kronentyp abhängig. Der Kronentyp wurde in dieser Regression nicht als potenzielle Variable berücksichtigt, da er den Zeitbedarf des Umgreifens nur mittelbar über die Anzahl der Umgreifvorgänge beeinflusst. Im Hinblick darauf, dass alle Modelle am Ende zu einem Gesamtmodell summiert werden sollten, wurde der Kronentyp nur einmal berücksichtigt, da er ansonsten (in der Summe) für ein und dieselbe Tätigkeit doppelt berücksichtigt gewesen wäre. Alternativ hätte ein genestetes, also ein aus zwei ineinander geschachtelten Modellen zusammengesetztes, hierarchisches Regressionsmodell angepasst werden können. Da die Modelle am Ende jedoch alle summiert werden sollten und damit ohnehin ineinander geschaltet wurden, wäre dieses komplexe Regressionsverfahren unverhältnismäßig gewesen. Außerdem wäre durch diese Vorgehensweise die praktische Interpretierfähigkeit des Teilmodells eingeschränkt worden. Der Einfluss des Kronentyps im Hinblick auf den Gesamtzeitverbrauch kann dadurch nicht vollständig repräsentiert werden, da ein Teil der Erklärungsstärke aus dem Modell gekürzt wurde. Bei der Schätzung dieser Aktivität zeigte sich, wie komplex die Abläufe innerhalb eines Prozesses ausfallen können. Eine weitere Besonderheit dieses Aktivitätsmodells stellt der starke Effekt des Harvestersystems dar (Formel 15), welcher vorrangig für die Effektstärke auf Prozessebene verantwortlich ist (Formel 18). Speziell in diesem Bereich benö- 


\section{Diskussion und Fazit}

tigt es daher weiterer Studien, die den Zeitbedarf dieser Aktivität und speziell den Einfluss des Harvestersystems tiefergehend analysieren.

Das Ablegen des Kronenrests wird ebenfalls durch den Kronentyp beeinflusst. Ist die Krone bzw. der Stamm verzweigt, wird es notwendig mehre Kronenrestteile separat abzulegen. Eine Besonderheit stellt die auftretende Zusatzaktivität dar, innerhalb derer der Kronenrest gezielt auf der Rückegasse abgelegt wird. Diese zusätzliche Bewegung erhöht den Zeitbedarf der Aktivität enorm. Für einen Baum mit einem Volumen von $0,5 \mathrm{~m}^{3}$ bedeutet dies beispielsweise einen Mehraufwand von rund $23 \%$ bezogen auf die gesamte Aufarbeitungszeit. Dieses Ergebnis unterstreicht noch einmal den enormen Einfluss der zusätzlichen Aktivitäten.

Mit dem Abschluss der kritischen Auseinandersetzung der einzelnen Aktivitäten kann zusammenfassend die Hypothese 1 bestätigt werden. Es zeigt sich aber, dass eine Einbeziehung von optionalen Aktivitäten (Umgreifen) methodische Schwierigkeiten birgt. Letztlich stellt die Bestätigung der Hypothese 1 eine Brücke zum Gesetz der Teilzeiten von Hilf (1941, S. 30) dar, wonach sich alle forstlichen Arbeiten in kleinere Tätigkeiten unterteilen lassen und diese einzeln von wenigen Faktoren abhängig sind.

H2: Die Produktivität der mechanisierten Buchenaufarbeitung steigt mit zunehmender Baumdimension bis zum Punkt der Leistungsgrenze an und sinkt im Zuge der negativen Grenzproduktivität danach wieder ab.

Grundsätzlich konnte das Auftreten einer Leistungsgrenze sowie einer negativen Grenzproduktivität für die mechanisierte Buchenaufarbeitung bestätigt werden. Die aktivitätsabhängige Regressionsanalyse konnte jedoch zeigen, dass eine negativ werdende Grenzproduktivität nicht durch alle Aktivitäten gleichermaßen, sondern ausschließlich durch die Fällung und das Entasten beeinflusst wird. Der überproportionale Zeitbedarf dieser beiden Aktivitäten über dem Baumvolumen ist maßgeblich für diesen Effekt verantwortlich. Darüber hinaus erhöht sich mit steigendem Baumvolumen die Wahrscheinlichkeit, dass innerhalb der Aufarbeitung umgegriffen werden muss und auch die Anzahl an notwendigen Umgreifvorgängen steigt an (Kapitel 7.1.5). In Abhängigkeit der Ausprägungen der restlichen Modellvariablen lässt sich der Punkt der maximalen Produktivität zwischen 2,1 und 2,4 $\mathrm{m}^{3}$ Baumvolumen bzw. 40 bis $50 \mathrm{~cm}$ BHD verorten (Abbildung 39). Dabei konnte kein Unterschied zwischen den eingesetzten Harvestersystemen für diesen 
Zusammenhang festgestellt werden, welcher durch die technische Vergleichbarkeit zwischen den Systemen zu erwarten war. Die Stärke der Leistungsabnahme oberhalb dieser Leistungsgrenze scheint im Vergleich mit den bestehenden Studien tendenziell niedriger auszufallen (Kapitel 7.2.4). Eine Erklärung dafür bietet die aktivitätsabhängige Modellierung. Wie der Vergleich mit dem direkt geschätzten Modell in Kapitel 7.2.5 zeigte, wird ein deutlich stärkerer quadratischer Zusammenhang geschätzt als aus der Analyse der Aktivitäten zu erwarten war. Dies würde sich in Form einer stärkeren Leistungsabnahme in der umgeformten Produktivitätsfunktion widerspiegeln und die wirkliche Leistungskurve daher verzerren. Darüber hinaus bedingen die unterschiedlichen Funktionstypen der Vergleichsmodelle in Kapitel 7.2.4 einen steileren Verlauf.

H3: Neben dem Baumvolumen bilden die Kronenform und die Qualität des Stammes wichtige Kostentreiber bei der mechanisierten Buchenaufarbeitung.

Bereits aus den Ergebnissen der Aktivitätsmodelle konnte abgeleitet werden, dass der Kronentyp und die Qualität eines Baumes einen erheblichen Einfluss auf die Aufarbeitungszeit besitzen. Obwohl diese beiden Variablen in einer Wechselwirkung miteinander stehen (Abbildung 32, nicht signifikant), zeigt die Analyse, wie wichtig es ist, beide Parameter getrennt voneinander zu erheben: Während die Stammqualität die Fällung und das Entasten beeinflusst, so trifft dies für den Kronentyp auf das Ablegen des Kronenrestes zu. Darüber hinaus hat der Kronentyp auch einen indirekten Effekt durch die Beeinflussung der Eintrittswahrscheinlichkeit und die Anzahl an Umgreifvorgängen während der Aufarbeitung. Durch die Kürzung der Variable Umgreifvorgänge aus dem Gesamtmodell wurde dieser Teil der Einflussstärke des Kronentyps aus dem Modell entfernt. Dass der Kronentyp dennoch einen erheblichen Einfluss auf die Produktivität beschreibt, zeigte sich in der Sensitivitätsanalyse (Abbildung 37). Damit konnte abschließend Hypothese H3 vollständig bestätigt werden. Die Stammqualität und der ausgebildete Kronentyp bilden neben dem Baumvolumen die wichtigsten morphologischen Kostentreiber für die mechanisierte Buchenaufarbeitung. 
H4: Eine steigende Komplexität der Aushaltung (Art und Anzahl der Sortimente) vermindert die Produktivität der mechanisierten Buchenaufarbeitung.

Die Überprüfung der Hypothese H4 hat sich innerhalb der Arbeit als besonders kompliziert herausgestellt. In bisherigen Forschungsarbeiten wurde hierbei die Anzahl und nicht die Sortenzusammensetzung analysiert. Je nach Datengrundlage wird somit entweder die Anzahl an Sortimenten pro Baum (Einzelbaummodelle; u. a. Nurminen et al., 2006, S. 344) oder die mittlere Sortenanzahl pro Hieb (Bestandesmodelle; u. a. Eriksson \& Lindroos, 2014, S. 185) als unabhängige Variable berücksichtigt. Zum Einfluss der Sortenzusammensetzung besteht dahingehend bis dato keine Evidenz. Dies ist allen voran den enormen Differenzierungsmöglichkeiten bei der Aushaltung geschuldet. Bereits bei vier festgelegten Sortimenten existieren theoretisch 15 unterschiedliche Aushaltungsoptionen an einem Entnahmebaum - sofern die Aushaltung von vier Sortimenten möglich ist. Ein Vergleich zwischen den Sortimenten innerhalb eines Einzelbaummodells lässt sich deshalb nur schwer quantifizieren.

Aus diesem Grund folgte die Analyse dem Vorgehen bestehender Arbeiten, indem die Anzahl ausgehaltener Sortimente als unabhängige Variable betrachtet wurde. Um dennoch Tendenzen über den Einfluss eines bestimmten Sortiments zu gewinnen, widmete sich Kapitel 7.1.4 dem Entasten auf Abschnittsebene. Aus diesen Ergebnissen können folgende Tendenzen abgeleitet werden: Je geringer die Qualitätsanforderungen eines Sortiments sind, desto höher ist der Grundzeitbedarf für das Entasten des Abschnittes. Die beiden qualitativ höherwertigen Sortimente (Palette und Stammholz) weisen jedoch einen zusätzlichen volumenabhängigen Zeitbedarf auf. Ein durchschnittlicher Palettenabschnitt benötigt Aufgrund seines höheren Volumens mehr Zeit für das Entasten als ein durchschnittlicher Industrieholzabschnitt. Darüber hinaus war es den Harvestersystemen nur möglich, Abschnitte mit einem Maximalvolumen von einem $\mathrm{m}^{3}$ aufzuarbeiten, ohne dass eine Zusatzaktivität notwendig wurde. Stärkere Abschnitte (ausschließlich Stammholz) mussten zusätzlich manipuliert werden.

Auf Baumebene zeigen die Ergebnisse, dass die Entscheidung über die Aushaltung einen grundsätzlichen Einfluss auf die Produktivität der mechanisierten Aufarbeitung hat (Abbildung 37). Mit jedem weiteren ausgehaltenen Sortiment am Baum sinkt die Produktivität - je nach Baumvolumen - um 2 bis $7 \%$. Dieser Mehraufwand fällt aber nicht grundsätzlich bei allen Bäumen eines Bestandes an, sondern wird nur bei denjenigen verursacht, bei denen eine weitere Sorte auch wirklich ausgehalten wird. Für die Einschätzung 
der Produktivität auf Bestandesebene (vgl. Kapitel 7.2.3) sollte daher eher die mittlere Anzahl an Sorten und nicht die maximale Anzahl genutzt werden. Um beispielsweise ein zweites Sortiment kostendeckend auszuhalten, müsste der Holzpreis des zweiten Sortiments in Abhängigkeit zum Baumvolumen zwischen 0,14 und 1,64€ pro $\mathrm{m}^{3}$ mehr erlösen als das erste Sortiment (Kosten = 192€/MAS; 44,625 \% Aufschlag allgemeiner Zeit). Für den Prozess der mechanisierten Aufarbeitung ist der Einfluss der Aushaltung aus rein monetärer Sicht daher als eher gering zu bewerten. Studienergebnisse weisen jedoch darauf hin, dass die Anzahl an ausgehaltenen Sortimenten aufgrund von käuferspezifischen Anforderungen tendenziell weiter zunehmen wird (Malinen et al., 2006, S. 90). Weiterhin ist anzumerken, dass die Art und Anzahl der Sortimente sowie deren Massenanfall einen deutlich größeren Effekt auf die vor- und nachgelagerten Prozesse der Holzbereitstellungskette besitzen (Kapitel 8.3).

H5a: Das Produktivitätsmodell dieser Arbeit lässt sich in die bestehenden Modelle der mechanisierten Buchen- bzw. Laubholzaufarbeitung einordnen.

Die Hypothese H5a bezieht sich auf den Vergleich des im Rahmen der Dissertation erarbeiteten Modells mit bereits bestehenden Modellen (Abbildung 39). Eine optische Verzerrung lässt sich durch die Schwierigkeit erklären, mehrdimensionale Modelle in zweidimensionalen Diagrammen darzustellen. Alle weiteren Variablen müssen mit (gemittelten) Daten eingesetzt werden, um nur die Abhängigkeiten der beiden Variablen von Interesse darzustellen. Nichtsdestotrotz zeigen sich hierbei - vor allem ab einem Baumvolumen von 0,5 $\mathrm{m}^{3}$ - deutliche Unterschiede. Speziell die Modelle von Guglhör (1994, S. 698) weisen in diesem Bereich eine höhere Leistung auf und erreichen hier bereits ihre Leistungsgrenze. Die Hypothese 5a konnte daher nur bedingt bestätigt werden. Die Differenzen zwischen den bisherigen Modellen und dem Produktivitätsmodell dieser Arbeit lassen sich vermutlich hauptsächlich durch den erheblich breiteren Gültigkeitsbereich und die Fokussierung auf neue und besonders leistungsstarke Harvestersysteme erklären, die auf die Aufarbeitung stärkerer Bäume ausgelegt sind (Tabelle 5). Damit liefert die Arbeit eine erste Antwort auf das Verhalten der mechanisierten Aufarbeitung bei Buchen über 1,5 $\mathrm{m}^{3}$ Baumvolumen (Anhang 2). Aufgrund der Neuartigkeit dieses Vorgehens sollten die Befunde zukünftig empirisch validiert werden. 
H5b: Die Produktivität der mechanisierten Buchenaufarbeitung ist geringer als in vergleichbaren Studien aus nadelholzdominierten Wäldern.

Die Hypothese 5b erweitert den Vergleichsrahmen auf Modelle, aus nadelholzdominierten Beständen. Auch in diesem Fall ist eine entstehende optische Verzerrung durch die zweidimensionale Darstellung mehrdimensionaler Modelle anzunehmen. Abbildung 40 verdeutlicht dennoch zweifelsfrei, dass der Produktivitätsverlauf der mechanisierten Nadelholzaufarbeitung deutlich über der festgestellten Leistungskurve dieser Arbeit liegt und dass, obwohl das dargestellte Buchenmodell unter der Annahme der produktivsten Bedingungen dargestellt wird. Es existieren dennoch Modelle, wie beispielsweise von Cremer (2008, S. 72), die eine vergleichbare Leistung beschreiben. Speziell im Bereich unter $0,4 \mathrm{~m}^{3}$ Baumvolumen lässt sich nur ein geringer Leistungsunterschied feststellen. Zusammenfassend konnte die Hypothese 5b, mit Ausnahme des Volumenbereichs unter $0,4 \mathrm{~m}^{3}$, bestätigt werden.

H6: Die separate Analyse der Aktivitäten offenbart signifikante Kostentreiber, die eine gemeinsame Analyse in Form eines Gesamtmodells des Aufarbeitungsprozesses nicht offenbart hätte.

Der angestellte Vergleich in Kapitel 7.2.5 konnte zeigen, welche Folgen für die Auswahl und Stärke der Kostentreiber entstehen, wenn die Modellierung nur auf Prozess- und nicht auf Aktivitätsebene durchgeführt wird. In der Analyse des Gesamtzeitverbrauchs gehen zum einen wichtige Kostentreiber verloren (Qualität, Anzahl der Sorten). Zum anderen wird die Stärke verbleibender Variablen anders eingeschätzt (Formel 18, Anhang 19). Nichtsdestotrotz ist das Endergebnis ein Modell, welches rein statistisch gesehen den Zeitverbrauch auf Prozessebene zufriedenstellend erklärt. Der Vergleich der Analysen macht aber deutlich, wie wichtig ein sachlicher Rahmen für ein analytisch korrektes Vorgehen ist. Als Grundlage für eine forstbetriebliche Entscheidungsfindung würde das entstandene Produktivitätsmodell sonst zu ungenauen Rückschlüssen führen. Die Hypothese 6 konnte somit vollständig bestätigt werden. 


\subsection{Ausblick}

Innerhalb einer Prozessanalyse sollte ein Prozess zwar immer isoliert untersucht werden, es ist jedoch mindestens genauso wichtig, die vor- und nachgelagerten Prozesse zu verstehen und zu berücksichtigen, da Abhängigkeiten zwischen diesen bestehen können (Kapitel 2.3). Kostentreiber, die für den untersuchten Prozess keine besondere Relevanz zeigten, können beispielweise die Produktivität eines vor- oder nachgelagerten Prozesses maßgeblich bestimmen (z. B. Anzahl der ausgehaltenen Sortimente). Aus diesem Grund sollen die präsentierten Ergebnisse an dieser Stellte im Hinblick auf die Holzbereitstellungskette eingeordnet werden und damit ein Blick ,über den Tellerrand“ dieser Arbeit hinaus erfolgen. Um ein besseres Verständnis der nachgelagerten Prozesse zu erhalten, werden an dieser Stelle zwei studentische Abschlussarbeiten in den Fokus gerückt, die unter enger fachlicher Betreuung durch den Autor dieser Dissertation in der Abteilung Forstökonomie der Georg-August-Universität Göttingen verfasst wurden. Die Ergebnisse dieser Abschlussarbeiten werden zusätzlich durch die Befunde weiterer Forschungsarbeiten ergänzt.

Leona Ottens (2019) schrieb ihre Bachelorarbeit über die „Prozessanalyse der Buchenholzrückung mittels Kombiforwarder“. Sie konnte zeigen, wie komplex der Prozess der Holzrückung ist, sobald mehrere Sortimente gleichzeitig gerückt werden. Dabei stellte sich das Stückvolumen auch für diesen Prozess als ein wichtiger Kostentreiber heraus. Weiterhin ließen sich Tendenzen bezüglich des starken Einflusses der Sortenanzahl auf die Produktivität der Buchenholzrückung erkennen, welche bereits in einer Studie von Manner et al. (2013, S. 14) umfassend beschrieben wurden. In dieser konnte zudem ein signifikanter Einfluss der Sortimentskonzentration ( $\mathrm{m}^{3}$ pro $100 \mathrm{~m}$ Gassenlänge) auf die Forwarderproduktivität nachgewiesen werden, welche maßgeblich durch die Aufarbeitung bestimmt wird. Ein weiteres interessantes Ergebnis bezieht sich auf das Vorrücken innerhalb der mechanisierten Aufarbeitung. Wie in der Studie von Väätäinen et al. (2006, S. 68) nachgewiesen werden konnte, reduziert sich die Produktivität eines Harvesters, sobald dieser die Abschnitte vorrückt. Dieses Vorgehen verbessert die Bedingungen für den Forwarder jedoch so enorm, dass sich die Produktivität - bezogen auf die gesamte Holzernte - dennoch erhöht. 


\section{Diskussion und Fazit}

Lucas Hebbecker (2019) schrieb seine Masterarbeit über die „Quantifizierung von Buchenrundholztransporten“ und trug damit zum tiefergehenden Verständnis über den Prozess und die Kostentreiber des Buchendirekttransportes bei. Die Arbeit konnte dabei nicht nur die Einflüsse des Sortiments, des Stückvolumens sowie der Polteranzahl für den Prozess quantifizierten - sie zeigte auch, wie besonders komplex sich die Abläufe innerhalb eines Prozesses darstellen können. Beide Abschlussarbeiten (Hebbecker, 2019; Ottens, 2019) konnten zeigen, wie eng die Prozesse der Holzbereitstellungskette miteinander verknüpft sind. Beispielsweise kann die Ausführung einer zusätzlichen Tätigkeit die Produktivität des untersuchten Prozesses senken, die Produktivität der Prozesskette jedoch erhöhen (z. B. Vorrücken des Harvesters). Diese Erkenntnisse zeigen, dass speziell in der Analyse der Prozesskette enorme Rationalisierungspotentiale enthalten sind, welche in zukünftigen Studien näher beleuchtet werden sollten. Zum Abschluss des Ausblickes soll nun der aufgezeigte Forschungsbedarf (Kapitel 8.1 \& 8.2) dieser Arbeit, bezogen auf die mechanisierten Aufarbeitung von Buche, zusammengefasst werden:

Erstens benötigt es weitere Einzelbaummodelle, um die Ergebnisse dieser Arbeit zu validieren. Es existieren keine Produktivitätsmodelle der mechanisierten Buchenaufarbeitung, die Bäume von mehr als 1,5 $\mathrm{m}^{3}$ Volumen integrieren und mit denen die Modelle dieser Arbeit verglichen werden können. Der notwendige Arbeitsaufwand kann durch die detailreichen Erkenntnisse dieser Arbeit reduziert werden (z. B. Variablenset). Darüber hinaus erscheint die Durchführung von sensorgestützten Zeitstudien gewinnbringend, um den Arbeitsaufwand speziell für die Zeitmessung weiter zu verringern. Zusätzlich sollten auch Bestandesmodelle entwickelt werden, welche die Aufarbeitung von stärker dimensionierten Buchenbeständen integrieren.

Zweitens konnte die vorliegende Arbeit Forschungslücken aufdecken, die ein umfassendes Verständnis des Prozesses erschweren. So fehlen beispielsweise Forschungsarbeiten, die sich auf die Quantifizierung der beschriebenen Zusatzaktivitäten konzentrieren. In dieser Arbeit konnte zwar erstmalig der Einfluss vieler dieser Aktivitäten quantifiziert werden - es benötigt aber noch Befunde dazu, welche Einflüsse diese Zusatzaktivitäten bedingen. Zu erwarten ist, dass speziell das Harvestersystem sowie der Maschinenführer diese Zusatzaktivitäten beeinflussen (Kapitel 4.4.3, Arbeitssystem). Für das Umgreifen konnten aus den Ergebnissen dieser Arbeit solche Aussagen zwar abgeleitet werden, dennoch konnten auch hier die Differenzen zwischen den Harvestersystemen nicht genauer erklärt werden.

Ein letzter Punkt betrifft abschließend die Korrekturfaktoren, die notwendig sind, um die 
Ergebnisse von arbeitszeitbasierten Studien auf die Gesamtarbeitszeit umzurechnen. Wie Kapitel 8.1.3 zeigte, werden diese Faktoren bereits in der Wissenschaft behandelt, es fehlt aber an systematischen Analysen, die diese Forschungslücke in den Fokus rücken. Für den Anwender wird dadurch die evidenzbasierte Auswahl passender Korrekturfaktoren erschwert.

\subsection{Schluss}

„Essentially, all models are wrong, but some are useful.“

Box \& Draper (1987, S. 424)

Modelle stellen immer nur ein vereinfachtes Abbild eines abgegrenzten Stücks der Wirklichkeit dar. Dennoch können gerade ebenjene Vereinfachungen der Realität - richtig angewandt - uns dabei helfen, verschiedene Prozesse zu erklären, zu verstehen, Schlüsse zu ziehen und vorauszusagen. Im Fokus dieser Arbeit stand die Analyse der prozessabhängigen Kosten der mechanisierten Buchenaufarbeitung im Rahmen eines forstlichen Produktivitätsmodells. Obwohl zahlreiche Modelle zur Erklärung der mechanisierten Aufarbeitungszeit existieren, beschränken sich diese zumeist auf Nadelholzbestände oder aber gelten nur für schwächere Buchen- bzw. Laubholzbestände. Dieses Vorgehen wurde kritisch hinterfragt, da der flächenmäßige Anteil an Buchenbeständen in Deutschland sowie deren durchschnittliche Vorräte und Bestandesalter tendenziell immer weiter ansteigen (BMEL, 2014, S. 16). Weiterhin erlauben die technischen Entwicklungen der letzten Jahre eine zunehmende Harvester-Nutzung auch in stärker dimensionierten Buchenbeständen (Kleinschmit, 2015, S. 43) - es fehlen jedoch Modelle, die den Prozess und speziell die prozessabhängigen Kosten der mechanisierten Buchenaufarbeitung genauer beschreiben, um eine forstbetriebliche Entscheidungsfindung zu unterstützen.

Genau an dieser Stelle setzte die vorliegende Arbeit an und integrierte das Prinzip des Stückvolumengesetzes in die Erstellung eines Produktivitätsmodells, wobei gezeigt werden konnte, dass die Erkenntnisse von Hilf (1928) auch für moderne forstliche Prozesse gelten. Die präsentierten Befunde dieser Arbeit lassen sich dabei wie folgt zusammenfassen: Die Produktivität der mechanisierten Buchenaufarbeitung wird vorrangig durch das Baumvolumen beeinflusst. Mit zunehmenden Baumvolumen steigt die Produktivität bis zum Punkt der Leistungsgrenze (40 bis $50 \mathrm{~cm}$ BHD) an und sinkt im Zuge der negativen 


\section{Diskussion und Fazit}

Grenzproduktivität danach wieder ab. Die Stammqualität und der ausgebildete Kronentyp bilden neben dem Baumvolumen die wichtigsten morphologischen Kostentreiber für die mechanisierte Buchenaufarbeitung. Eine steigende Komplexität der Aushaltung vermindert die Aufarbeitung nur geringfügig, führt aber zu deutlich stärkeren Produktivitätseinbußen für die vor- und nachgelagerten Prozesse. Weiterhin erscheint es dienlich, neben einem Gesamtmodell des Aufarbeitungsprozesses die einzelnen Aktivitäten separat zu analysieren, um auf diese Weise zusätzliche signifikante Kostentreiber zu identifizieren. Die Ergebnisse zeigen - insbesondere vor dem Hintergrund des zunehmenden Anteils an Buchenbeständen in den deutschen Wäldern - die große Relevanz sowie den Bedarf von Forschungsarbeiten zu dieser Thematik. Die Arbeit hat verdeutlicht, dass es gewinnbringend ist, forstliche Prozesse im Detail zu analysieren, da sich speziell in der Analyse der einzelnen Aktivitäten die wirkliche Stärke der Kostentreiber identifizieren lässt. Ein besonderes Augenmerk zukünftiger Forschungsarbeiten sollte im Besonderen auf den Einfluss des Harvestersystems, speziell im Kontext auftretender Zusatzaktivitäten, gerichtet werden. Zusammenfassend lässt sich festhalten, dass die Dissertation einen ersten Schritt zur Beantwortung der bestehenden Forschungslücken darstellte. Gemäß dem Anspruch von Box und Draper (1987) liefert sie ein nützliches Modell, dass sowohl in der Theorie als auch Praxis sinnvoll zur Vorhersage der prozessabhängigen Kosten der mechanisierten Buchenaufbereitung genutzt werden kann. 


\section{Literaturverzeichnis}

Alam, M., Walsh, D., Strandgard, M. \& Brown, M. (2014). A log-by-log productivity analysis of two Valmet 475EX harvesters. International Journal of Forest Engineering, 25(1), 14-22.

Altherr, E., Unfried, P. \& Hrahetzky, V. (1974). Statistische Rindenbeziehungen als Hilfsmittel zur Ausformung und Ausmessung unentrindeten Stammholzes: Teil I Kiefer, Buche, Hainbuche und Roterle. In FVA Baden-Württemberg (Hg.), Mitteilungen der Forstlichen Versuchs- und Forschungsanstalt Baden-Württemberg (S. 61-137). FVA Baden-Württemberg.

Arbeitsgemeinschaft Deutscher Waldbesitzerverbände [AGDW] e.V. (2020). Unternehmen Wald. Zuletzt abgerufen am 27.10.2020 von https://www.waldeigentuemer.de/themen/unternehmen-wald/

Arbeitsgemeinschaft forstwirtschaftlicher Lohnunternehmer Niedersachsen e. V. (2017). AFL-INFO 2016/2017. AfL Niedersachsen e.V.

Asikainen, A. (1995). Discrete-event simulation of mechanized wood-harvesting systems (Dissertation). Universität Joensuu.

Bacher-Winterhalter, M. (2003). Mechanisierte Starkholzaufarbeitung in strukturreichen Mischbeständen. Forsttechnische Informationen, 9, 93-99.

Bacher-Winterhalter, M. (2004). Optimierungsmöglichkeiten und Restriktionen eines mechanisierten Holzerntesystems bei der Umsetzung moderner Waldbaukonzepte am Beispiel des Südschwarzwaldes (Dissertation). Albert-Ludwigs-Universität Freiburg im Breisgau.

Bauer, J. W. (2006). Verbesserung der Logistik in der Wertschöpfungskette zwischen Kleinprivatwald und Holzwirtschaft mittels Methoden des Business Process Reengineering (Dissertation). Technische Universität München.

Baumann, T. (2008). Analyse logistischer Prozesse und deren Optimierungspotentiale entlang der Holzbereitstellungskette vom Wald zum Werk unterstützt durch spezielle Verfahren der Prozessmodellierung: Durchgeführt am Beispiel verschiedener Forst- und Holzbetriebe in der Region Ostalb (Dissertation). Albert-Ludwigs-Universität Freiburg im Breisgau.

Baumeister, B. \& von Bodelschwingh, E. (2004). Fallstudie zur physischen und psychischen Belastung an Harvesterarbeitsplätzen. Forst und Holz, 59(6), 291-294. 
Literaturverzeichnis

Baumgarten, H. \& Wiegand, A. (1996). Optimale Logistik durch Prozesskettenmanagement. Industrie Management, 12(3), 53-56.

Beach, D. \& Pedersen, R. B. (2019). Process-tracing methods: Foundations and guidelines. University of Michigan Press.

Becher, G. (2015). Clusterstatistik Forst und Holz: Tabellen für das Bundesgebiet und die Länder 2000 bis 2013. Thünen Working Paper 67. Johann Heinrich von ThünenInstitut.

Becker, G. (1997). Naturverträgliche Nutzung des Waldes: Naturverträgliche und rationelle Rohholzbereitstellung. Schriftenreihe des Dachverbandes der wissenschaftlichen Gesellschaften der Agrar-, Forst-, Ernährungs-, Veterinär-und Umweltforschung e.V., 27, 76-84.

Bergel, D. (1973). Formzahluntersuchungen an Buche, Fichte, europäischer Lärche und japanischer Lärche zur Aufstellung neuer Massentafeln. Allgemeine Forst- und Jagdzeitung, 5/6, 117-124.

Bernroider, E. \& Stix, V. (2004). Grundzüge der Modellierung: Anwendungen für die Softwareentwicklung. Facultas.

Borcherding, M. (2007). Rundholztransportlogistik in Deutschland - eine transaktionskostenorientierte empirische Analyse (Dissertation). Universität Hamburg.

Börner, M., Guericke, M., Leder, B., Nutto, L., Stähr, F. \& Weinreich, F. (2003). Erhebung qualitätsrelevanter Parameter am Einzelbaum - Aufnahmestandards für junge bis mittelalte Laubhölzer als Grundlage wissenschaftlicher Untersuchungen. Forstarchiv, 74, 275-282.

Borr, U. \& Pfeuffer, A. (1999). Bestandesvorbereitung in der mechanisierten Holzernte. Auswirkungen auf Leistung, Kosten und Waldbau. Forsttechnische Informationen, 10, 88-91.

Bort, U., Mahler, G. \& Pfeil, C. (1993). Mechanisierte Holzernte. Forsttechnische Informationen, 11, 121-124.

Böttcher, F. \& Husmann, K. (2020). Digitale Warenwirtschaft in deutschen Forstbetrieben: Einführung eines bundesweit einheitlichen Datenstandards ist an vielen Betrieben vorbeigegangen. Holz-Zentralblatt, 9, 178.

Böttcher, F., von Plettenberg, F. \& Husmann, K. (2018). Analyse der vollmechanisierten Aufarbeitung von Buche. AFZ-DerWald, 73(5), 27-29.

Box, G. E. P. \& Draper, N. R. (1987). Empirical model-building and response surfaces. Wiley. 
Bruchner, A.-K. (2004). Industrielle Simulationssoftware als Entscheidungsunterstützungsinstrument in der Produktionslogistik der Holzerntekette (Dissertation). Technische Universität München.

Bundesministerium für Ernährung und Landwirtschaft [BMEL] (2014). Der Wald in Deutschland. Ausgewählte Ergebnisse der dritten Bundeswaldinventur. BMEL.

Bundesministerium für Ernährung und Landwirtschaft [BMEL] (2016). Ergebnisse der Bundeswaldinventur 2012. BMEL.

Bundesministerium für Ernährung und Landwirtschaft [BMEL] (2020). Dritte Bundeswaldinventur (2012). Zuletzt abgerufen am 28.10.2020 von https://bwi.info/

Busch, A. \& Dangelmaier, W. (2002). Integriertes Supply Chain Management - ein koordinationsorientierter Überblick. In A. Busch \& W. Dangelmaier (Hg.), Theorie und Praxis effektiver unternehmensübergreifender Geschäftsprozesse (Seite 1-21). Gabler Verlag.

Coenenberg, A. G. (1997). Kostenrechnung und Kostenanalyse. Verlag Moderne Industrie.

Cremer, T. (2008). Bereitstellung von Hackschnitzeln durch die Forstwirtschaft (Dissertation). Albert-Ludwigs-Universität Freiburg im Breisgau.

Danilović, M., Tomašević, I. \& Gačić, D. (2011). Efficiency of John Deere 1470D ECOIII Harvester in Poplar Plantations. Croatian Journal of Forest Engineering, $32(2), 533-548$.

Deutscher Forstwirtschaftsrat [DFWR] e.V. (2012). Produktplan Forst. Zuletzt abgerufen am 28.10.2020 von https://www.dfwr.de/images/PDFs/AfB/1.1_Anlagen.pdf

Deutscher Forstwirtschaftsrat [DFWR] e.V. \& Deutscher Holzwirtschaftsrat [DHWR] e.V. (2020). Rahmenvereinbarung für den Rohholzhandel in Deutschland (RVR). Zuletzt abgerufen am 28.10.2020 von https://v2.verband-crm.de/dateien/917288/rahmenvereinbarung+f\%C3\%BCr+den+rohholzhandel+in+deutschland $+\%$ $28 \mathrm{rvr} \% 29$

Dieler, J. \& Pretzsch, H. (2013). Morphological plasticity of European beech (Fagus sylvatica L.) in pure and mixed-species stands. Forest Ecology and Management, 295, 97-108.

Döring, P., Glasenapp, S. \& Mantau, U. (2017a). Holz- und Zellstoffindustrie 2015. Zwischenbericht des Teilvorhabens Entwicklung der Produktionskapazität und Holzrohstoffnutzung. Zuletzt abgerufen am 28.10.2020 von https://literatur.thuenen.de/digbib_extern/dn058689.pdf 
Literaturverzeichnis

Döring, P., Glasenapp, S. \& Mantau, U. (2017b). Rohstoffmonitoring Holz. Holzwerkstoffindustrie 2015. Entwicklung der Produktionskapazität und Holzrohstoffnutzung. Abschlussbericht des Teilvorhabens Entwicklung der Produktionskapazität und Holzrohstoffnutzung. Zuletzt abgerufen am 28.10.2020 von https://literatur.thuenen.de/digbib_extern/dn058690.pdf

Döring, P., Glasenapp, S. \& Mantau, U. (2017c). Rohstoffmonitoring Holz. Sägeindustrie 2015. Einschnitt- und Produktionsvolumen. Zwischenbericht des Teilvorhabens Sägeindustrie 2015 - Einschnitt- und Produktionsvolumen. Zuletzt abgerufen am 28.10.2020 von https://literatur.thuenen.de/digbib_extern/dn058688.pdf

Dummel, K., Scharnagl, G. \& Schwanitz, P. (1995). Entlohnung. Geschichte und Zukunft des EST. Forsttechnische Informationen, 47, 121.

Eliasson, L. (1999). Simulation of thinning with a single-grip harvester. Forest Science, 45(1), 26-34.

Ellenberg, H. \& Dierschke, H. (2010). Vegetation Mitteleuropas mit den Alpen: In ökologischer, dynamischer und historischer Sicht. Verlag Eugen Ulmer.

Ellram, L. \& Cooper, M. (1990). Supply Chain Management. Partnerships and the Shipper-Third- Party Relationship. International Journal of Logistics Management, $1(2), 1-10$.

Elsasser, P., Altenbrunn, K., Köthke, M., Lorenz, M. \& Meyerhoff, J. (2020). Regionalisierte Bewertung der Waldleistungen in Deutschland. Johann Heinrich von Thünen-Institut.

Engelmann, T. (1995). Business Process Reengineering: Grundlagen, Gestaltungsempfehlungen, Vorgehensmodell. Deutscher Universitätsverlag.

Engler, B. (2011). Bewirtschaftung von Eukalyptusplantagen in China (Dissertation). Alberts-Ludwig-Universität Freiburg im Breisgau.

Eriksson, M. \& Lindroos, O. (2014). Productivity of harvesters and forwarders in CTL operations in northern Sweden based on large follow-up datasets. International Journal of Forest Engineering, 25(3), 179-200.

Erler, J. (2000). Forsttechnik: Verfahrensbewertung. Ulmer.

Erler, J. \& Dög, M. (2009). Funktiogramme für Holzernteverfahren: Komplex und trotzdem gut verständlich. Forsttechnische Informationen, 9/10, 14-17.

Erni, V. \& Frutig, F. (2004). „HeProMo“ - für die einfache Vorkalkulation von Holzerntearbeiten. Wald und Holz, 85(5), 46-47. 
Erni, V., Frutig, F., Holm, S., Lemm, R., Pedolin, D. \& Thees, O. (2004). Kalkulation von Holzerntearbeiten: Das Produktivitätsmodell. Zuletzt abgerufen am 28.10.2020 von www.waldwissen.net

Erteld, W. \& Achterberg, W. (1954). Narbenbildung, Qualitätsdiagnose und Ausformung bei der Rotbuche. Archiv für das Forstwesen, 3, 577-619.

Faes, C., Ormerod, J. T. \& Wand, M. P. (2011). Variational Bayesian inference for parametric and nonparametric regression with missing data. Journal of the American Statistical Association, 106(495), 959-971.

Fahrmeir, L., Kneib, T., Lang, S. \& Marx, B. (2013). Regression. Springer.

Feierabend, R. (1980). Beitrag zur Abstimmung und Gestaltung unternehmensübergreifender logistischer Schnittstellen. Schriftenreihe der Bundesvereinigung Logistik e.V.

Feil, P., Neitzel, C., Seintsch, B. \& Dieter, M. (2018). Privatwaldeigentümer in Deutschland: Ergebnisse einer bundesweiten Telefonbefragung von Personen mit und ohne Waldeigentum. Landbauforschung, 68, 87-130.

Fischer, C., Husmann, K. \& Möhring, B. (2018). Quantifizierung des Waldumbaus in Niedersachsen. AFZ-DerWald, 13, 28-30.

Fischer, C. \& Stadelmann, G. (2019). Calculation of potential timber harvesting costs (HeProMo). In C. Fischer \& B. Traub (Hg.), Managing Forest Ecosystems. Swiss National Forest Inventory - methods and models of the fourth assessment (S. 257-263). Springer International Publishing.

Fischer, M. L. (1997). What is the right supply chain for your product? Harvard Business Review, 75, 105-116.

Forbrig, A. (2000). Konzeption und Anwendung eines Informationssystems über Forstmaschinen auf der Grundlage von Maschinenbuchführung, Leistungsnachweisen und technischen Daten (Dissertation). Technische Universität München.

Forstliche Versuchs- und Forschungsanstalt Baden-Württemberg [FVA-BW] (2001). Güteansprache am stehenden Stamm: Arbeitsanweisung für die Güteansprache am stehenden Stamm im Rahmen der 2. Bundeswaldinventur (BWI 2) in Baden-Württemberg. Zuletzt abgerufen am 28.10.2020 von https://www.fva-bw.de/daten-undtools/forschung/gueteansprache-am-stehenden-stamm

Gadow, K. von. (2003). Waldstruktur und Wachstum: Beilage zur Vorlesung im Wintersemester 2003/2004. Universitätsdrucke. Universitätsverlag Göttingen. 
Literaturverzeichnis

Gellerstedt, S. (1997). Mechanised cleaning of young forest - The strain on the operator. International Journal of Industrial Ergonomics, 20(2), 137-143.

Gerasimov, Y., Seliverstov, A. \& Syunev, V. (2012). Industrial round-wood damage and operational efficiency losses associated with the maintenance of a single-grip harvester head model: A case study in Russia. Forests, 3(4), 864-880.

Glöde, D. \& Sikström, U. (2001). Two felling methods in final cutting of shelterwood, single-grip harvester productivity and damage to the regeneration. Silva Fennica, 35(1), 71-83.

Grönlund, Ö. \& Eliasson, L. (2019). Birch shelterwood removal - harvester and forwarder time consumption, damage to understory spruce and net revenues. International Journal of Forest Engineering, 30(1), 26-34.

Guglhör, W. (1994). Durchforstung von Buchenbeständen mit einem Kranvollernter. AFZ-DerWald, 49(13), 695-698.

Guglhör, W. (1995). Pflegliche Durchforstung mit Holzerntemaschinen. Abschlussbericht zum Projekt V25. Bayerische Landesanstalt für Wald und Forstwirtschaft.

Gunnarsson, P. \& Hellström, C. (1992). Bestånd med underväxt rätt åtgard på rätt plats sänker kostnaderna. Forskningsstiftelsen Skogsarbeten. Redogörelse, 1, 65-68.

Gutenberg, E. (1951). Grundlagen der Betriebswirtschaftslehre: Die Produktion. Springer.

Häberle, S. (1984). Standardisierung zweidimensionaler Ausgleichsfunktionen über Richtgrad und Richtkonstante. Forstarchiv, 55(4), 220-225.

Hallé, F., Oldeman, R. A. A., Tomlinson, P. B. \& Hallé-Oldeman. (1978). Tropical trees and forests: An architectural analysis. Springer.

Hamberger, J. (2003). Wie Mechanisierung und Umweltvorsorge die Forstwirtschaft veränderten. LWF aktuell, 39, 33-36.

Hansen, J., Nagel, J., Schmidt, M. \& Spellmann, H. (2008). Das mittelfristige Buchenholzaufkommen in Niedersachsen und Deutschland. In Nordwestdeutsche Forstliche Versuchsanstalt (Hg.), Beiträge aus der Nordwestdeutschen Forstlichen Versuchsanstalt. Ergebnisse angewandter Forschung zur Buche (S. 291-310). Universitätsverlag Göttingen.

Hapla, F. \& Militz, H. (2008). Verwertung und Verwendung von Buchenholz. In Nordwestdeutsche Forstliche Versuchsanstalt (Hg.), Beiträge aus der Nordwestdeutschen Forstlichen Versuchsanstalt. Ergebnisse angewandter Forschung zur Buche (S. 311-326). Universitätsverlag Göttingen. 
Harrington, L. (1995). Logistics: agent for change - Shaping the integrated supply chain. Transportation and Distribution Management, 15(1), 30-34.

Harstela, P. (2004). The competence of the forest-machine operator and tacit knowledge, Studienunterlagen der University of Joensuu. Zuletzt abgerufen am 28.10.2020 von http://matwww.ee.tut.fi/simumedia_www/Seminaari/Presentations/Harstela_presentation.pdf

Hebbecker, L. (2019). Quantifizierung von Buchenrundholztransporten (Masterarbeit). Georg-August-Universität Göttingen.

Hegetschweiler, T. (1988). Grundlagen zur Kosten- und Investitionsbeurteilung bei der mittelfristigen Nutzungsplanung des Forstbetriebes (Dissertation). ETH Zürich.

Heide, H. C., von der Frieder, S. \& Bombosch, F. (2005). Kalkulationsmodul auf Basis einer Harvesterleistungsanalyse. AFZ-DerWald, 18, 962-964.

Hein, S. (2007). Ansätze zur Modellierung der Überwallung und inneren Ästigkeit bei Buche (Fagus sylvatica L.). Konferenzpapier der Jahrestagung Deutscher Verband Forstlicher Forschungsanstalten: Sektion Ertragskunde.

Heindl, U. \& Pausch, R. (2007). Nadelstarkholz erwünscht. LWF aktuell, 59, 6-7.

Heinimann, H. (2001). Productivity of a cut-to-length harvester family -an analysis based on operation data. Beitrag auf dem Council on Forest Engineering (COFE) Conference Proceedings: “Appalachian Hardwoods: Managing Change”, Snowshoe, July 15-18, 2001.

Hemm, M., Ziesak, M. \& Warkotsch, W. (2006). Simulating harvesting procedures to evaluate different working systems based on dicrete single tree events. Beitrag auf dem Proceedings of the International Precision Forestry Symposium, Stellenbosch University, South Africa.

Hengst, E. (1964). Der Kronenbau der Buche im Erzgebirge. Forstwissenschaftliches Centralblatt, 83(3-4), 79-87.

Hilf, H. H. (1928). Die Arbeitsleistung im Hauungsbetrieb. Nicht veröffentlichte Akte des Instituts für forstliche Arbeitswissenschaft, Eberswalde.

Hilf, H. H. (1941). Die Erforschung und Verbesserung der Waldarbeit. Vortragsunterlagen an der Forstlichen Hochschule Stockholm. Hannover.

Hilf, H. H., Ries, L. W. \& Strehlke, E. G. (1927). Forstliche Arbeitswissenschaft: Vortragsunterlagen für Vorträge im Deutschen Forstverein in Rostock am 25.08.1927.

Hill, W., Fehlbaum, R. \& Ulrich, P. (1994). Ziele, Instrumente und Bedingungen der Organisation sozialer Systeme. Haupt. 
Literaturverzeichnis

Höfle, H. H. (2002). Zum Stand und zu den Tendenzen der Forsttechnik. Holz-Zentralblatt, 77, 939.

Horváth, P. \& Mayer, R. (1989). Prozeßkostenrechnung. Controlling, 1(4), 214-219.

Horváth, P. \& Renner, A. (1990). Prozeßkostenrechnung: Konzept, Realisierungsschritte und erste Erfahrungen. Fortschrittliche Betriebsführung und Industrial Engineering, 39(3), 100-107.

Horváth, P. (2011). Controlling. Vahlen.

Hossiep, R. (2019). Soziale Erwünschtheit. Dorsch Lexikon der Psychologie. Zuletzt abgerufen am 28.10.2020 von https://dorsch.hogrefe.com/stichwort/soziale-erwuenschtheit

Hostettler, M. (2002). Die waldpolitische Öffnung: Ein Holzweg? Schweizerische Zeitschrift für Forstwesen, 153(2), 59-67.

Houlihan, J. B. (1988). International supply chains: A new approach. Management Decision, 26(3), 13-19.

Hug, J. (2004). Optimierung von Geschäftsprozessen in der Forstwirtschaft durch den Einsatz von Informationstechnologie - am Beispiel der Holzbereitstellung auf Revierebene (Dissertation). Albert-Ludwigs-Universität Freiburg im Breisgau.

Husmann, K. (2013). Prognose ökonomisch optimaler Nutzungsintensität von Buchenkronen: (Masterarbeit). Georg-August-Universität Göttingen.

Husmann, K., Auer, V., Beitzen-Heneke, I., Bischoff, H., Fehrensen, W.-G., Fischer, C., Gilly, A., Pflüger-Grone, H., Nagel, J., Spellmann, H. \& Zscheile, M. (2016). Mittelfristigem Anstieg folgt stetiger Rückgang - Zustand und Entwicklung der Rohholzverfügbarkeit in der buchenreichen Mitte Deutschlands. Holz-Zentralblatt, 37, 899-901.

Imboden, D. M. \& Koch, S. (2003). Systemanalyse: Einführung in die mathematische Modellierung natürlicher Systeme. Springer.

Iske, A. (2004). Thinning algorithms. In A. Iske (Hg.), Lecture Notes in Computational Science and Engineering: Multiresolution Methods in Scattered Data Modelling (S. 67-125). Springer.

Jacke, H. (1980). Zur Technik der Regressionsrechnung bei der Analyse forstlicher Arbeitszeitstudien (Dissertation). Georg-August-Universität Göttingen.

Jaeger, D. (2020). Vorlesungsunterlagen zum Modul Holzernte und Logistik: Teilmodul 1: Holzernteverfahren. Georg-August-Universität Göttingen. 
Jiroušek, R., Klvač, R. \& Skoupý, A. (2007). Productivity and costs of the mechanised cut-to-length wood harvesting system in clear-felling operations. Journal of Forest Science, 53(10), 476-482.

Jöbstl, H. (2000). Kosten- und Leistungsrechnung in Forstbetrieben. Österreichischer Agrarverlag.

Jossé, G. (2012). Basiswissen Kostenrechnung: Kostenarten, Kostenstellen, Kostenträger, Kostenmanagement. C. H. Beck.

Kashani, M. H. \& Dinpashoh, Y. (2012). Evaluation of efficiency of different estimation methods for missing climatological data. Stochastic Environmental Research and Risk Assessment, 26(1), 59-71.

Kärhä, K., Rönkkö, E. \& Gumse, S.-I. (2004). Productivity and cutting costs of thinning harvesters. International Journal of Forest Engineering, 15(2), 43-56.

Keller, G., Scheer, A.-W. \& Nüttgens, M. (1992). Semantische Prozeßmodellierung auf der Grundlage“Ereignisgesteuerter Prozeßketten (EPK)“. Instituts für Wirtschaftsinformatik, Universität des Saarlandes.

Kellogg, L. D. \& Bettinger, P. (1994). Thinning productivity and cost for a mechanized cut-to-length system in the northwest pacific coast region of the USA. Journal of Forest Engineering, 5(2), 43-54.

Kleinschmit, C. (2015). Die ökonomisch optimale Aufarbeitungsintensität. Universitätsverlag Göttingen.

Koepke, D. \& Hecker, M. (1998). Herleitung von Planungskosten für die Unterstützung mittelfristiger Entscheidungen im Forstbetrieb. Forst und Holz, 53(15), 467-471.

König \& Schreiber. (1997). Die Einführung der Prozeßkostenrechnung in der Bayerischen Staatsforstverwaltung. Forstökonomisches Kolloquium, Schloss Nienover bei Göttingen.

Kraft, G. (1884). Beiträge zur Lehre von Durchforstungen, Schlagstellungen und Lichtungshieben. Klindworth.

Krahl-Urban, J. (1953). Baumtypen bei Eichen und Buchen. Allgemeine Forstzeitschrift, $8(20), 246-248$.

Krahl-Urban, J. (1955). Über Erziehungsmaßnahmen in Buchenbeständen. Allgemeine Forstzeitschrift, 10(35/36), 401-406.

Krahl-Urban, J. (1962). Buchen-Nachkommenschaften. Allgemeine Forst- und Jagdzeitung, 133, 29-38. 
Literaturverzeichnis

Krell, M. (2006). Leistungsuntersuchungen am Raupenharvester Valmet 911.1 X3M auf der Basis des Datenerfassungsprogramms „MaxiHarvester“ (Diplomarbeit). TU Dresden.

Kroth, W. \& Bartelheimer, P. (1993). Holzmarktlehre. Parey.

Krupp, T. (2006). Benchmarking als Controlling-Instrument für die Kontraktlogistik: Prozessbenchmarking für Logistikdienstleister am Beispiel von Lagerdienstleistungen. Eul-Verlag.

Kuhn, A. \& Hellingrath, B. (2002). Supply Chain Management: Optimierte Zusammenarbeit in der Wertschöpfungskette. Springer.

Labelle, E. \& Huss, L. (2018). Creation of value through a harvester on-board bucking optimization system operated in a spruce stand. Silva Fennica, 52(3), 9947.

Labelle, E. \& Jaeger, D. (2011). Soil compaction caused by cut-to-length forest operations and possible short-term natural rehabilitation of soil density. Soil Science Society of America Journal, 75(6), 2314-2329.

Labelle, E., Soucy, M., Cyr, A. \& Pelletier, G. (2016). Effect of tree form on the productivity of a cut-to-length harvester in a hardwood dominated stand. Croatian Journal of Forest Engineering, 37(1), 175-183.

Lackes, R., Siepermann, M. \& Schewe, G. (2018). Geschäftsprozess. Gabler Wirtschaftslexikon. Zuletzt abgerufen am 28.10.2020 von https://wirtschaftslexikon.gabler.de/definition/geschaeftsprozess-35399/version-258881

Lange, F. (2006). Prämissen für Produktivitätsvergleiche zwischen Vollerntern über standardisierte Datensätze (Diplomarbeit). Georg-August Universität Göttingen.

Liepiņš, K., Lazdiņš, A., Liepiņš, J. \& Prindulis, U. (2015). Productivity and cost-effectiveness of mechanized and motor-manual harvesting of Grey Alder (Alnus incana (L.) Moench): A case study in Latvia. Small-scale Forestry, 14(4), 493-506.

Lindroos, O., Bergström, D., Johansson, P. \& Nordfjell, T. (2008). Cutting corners with a new crane concept. International Journal of Forest Engineering, 19(2), 21-27.

Löffler. (1992). Arbeitswissenschaft für Studierende der Forstwissenschaft. Manuskript zu den Lehrveranstaltungen am Lehrstuhl für Forstliche Arbeitswissenschaft und Angewandte Informatik, Ludwigs-Maximilians-Universität München.

Lüthy, C. (1997). Holzrücken mit Forwarder. Wald und Holz, 4, 33-35.

Magagnotti, N., Kanzian, C., Schulmeyer, F. \& Spinelli, R. (2013). A new guide for work studies in forestry. International Journal of Forest Engineering, 24(3), 249-253. 
Magagnotti, N., Spinelli, R., Acuna, M., Bigot, M., Guerra, S., Hartsough, B., Kanzian, C., Kärhä, K., Lindroos, O., Roux, S., Talbot, B., Esteban, E. \& Zormaier, F. (2012). Good practice guidelines for biomass production studies, COST Action FP-0902, WG 2 Operations research and measurement methodologies. CNR IVALSA.

Malinen, J., Kilpeläinen, H., Wall, T. \& Verkasalo, E. (2006). Variation in the value recovery when bucking to alternative timber assortments and log dimensions. Forestry Studies, 45, 89-100.

Malinen, J., Taskinen, J. \& Tolppa, T. (2018). Productivity of cut-to-length harvesting by operators' age and experience. Croatian Journal of Forest Engineering, 39, 15-22.

Manner, J., Nordfjell, T. \& Lindroos, O. (2013). Effects of the number of assortments and $\log$ concentration on time consumption for forwarding. Silva Fennica, 47(4), 1030.

Mantau, U. (2018). Rohstoffmonitoring Holz - Erwartungen und Möglichkeiten. Fachagentur Nachwachsende Rohstoffe e. V. (FNR).

Mederski, P., Bembenek, M., Karaszewski, Z., Łacka, A., Szczepańska-Álvarez, A. \& Rosińska, M. (2016). Estimating and modelling harvester productivity in pine stands of different ages, densities and thinning intensities. Croatian Journal of Forest Engineering, 37, 27-36.

Michels, L. (2017). Die Ermittlung von Unfallschwerpunkten in der Forstwirtschaft (Dissertation). Technische Universität Dresden.

Miller, J. \& Vollmann, T. (1985). The hidden factory. Harvard Business Review, 1985(63), 142-150.

Möhring, B. (1994). Über ökonomische Kalküle für forstliche Nutzungsentscheidungen: Ein Beitrag zur Förderung des entscheidungsorientierten Ansatzes der forstlichen Betriebswirtschaftslehre. Sauerländer.

Möhring, B., Leefken, G. \& Gutsche, C. (2008). Betriebswirtschaftliche Bewertung von Buchenwäldern. In Nordwestdeutsche Forstliche Versuchsanstalt (Hg.), Beiträge aus der Nordwestdeutschen Forstlichen Versuchsanstalt. Ergebnisse angewandter Forschung zur Buche (S. 327-341). Universitätsverlag Göttingen.

Möhring, B. \& Wilhelm, S. (2012). Der Forstliche Betriebsvergleich Westfalen-Lippe. Die Waldbauern in NRW, 6, 16-18

Moog, M. (1995). Die Prozeßkostenrechnung in Forstbetrieben: Eine geeignete Form der Plankostenrechnung zur Unterstützung mittelfristiger Produktionsentscheidungen. Forst und Holz, 50(3), 69-74. 
Literaturverzeichnis

Morat, J. (2005). KWF auf der LIGNA 2005. Prozesskette Holz im Mittelpunkt - „Runde Tische“ auf der LIGNA. Forsttechnische Informationen, 57(6), 73-76.

Morat, J., Forbig, A. \& Graupner, J. (1998). Holzernteverfahren: Vergleichende Erhebung und Beurteilung der Holzernteverfahren in der BRD. KFW.

Mrosek, T., Kies, U. \& Schulte, A. (2005a). Clusterstudie Forst und Holz Deutschland 2005: Forst- und Holzwirtschaft hat sehr große volkswirtschaftliche und arbeitsmarktpolitische Bedeutung. Holz-Zentralblatt, 84, 1113-1117.

Mrosek, T., Kies, U. \& Schulte, A. (2005b). Neue Erkenntnisse im Rahmen der Clusterstudie Forst und Holz Deutschland. Privatwaldbesitz in Deutschland. AFZDerWald, 22, 1211-1213.

Nåbo, A. (1990). Skogsmaskinförarens arbetsbelastning: Studier av arbete i röjning, gallring och slutavverkning. Sveriges lantbruksuniversitet Institutionen för skogsteknik.

Nagel, J. \& Biging, G. S. (1995). Schätzung der Parameter der Weibullfunktion zur Generierung von Durchmesserverteilungen. Allgemeine Forst- und Jagdzeitung, 166(9/10), 185-189.

Nagel, M. \& Böttcher, F. (2019). Neue Ansätze für eine forstliche Warenwirtschaft. AFZDerWald, 74(20), 46-47.

Nakagawa, M., Hamatsu, J., Saitou, T. \& Ishida, H. (2007). Effect of tree size on productivity and time required for work elements in selective thinning by a harvester. International Journal of Forest Engineering, 18(2), 24-28.

Neugebauer, K. J. (2013). Analyse des Prozesses und der Genauigkeit der Harvestervermessung in der hochmechanisierten Buchenernte: Ein Fallbeispiel aus dem Niedersächsischen Forstamt Münden (Bachelorarbeit). Georg-August-Universität Göttingen.

Nicholls, A., Bren, L. \& Humphreys, N. (2004). Harvester productivity and operator fatigue: Working extended hours. International Journal of Forest Engineering, 15(2), $57-65$.

Nick, L. \& Forbrig, A. (2002). Forsttechnikerhebung - Stand, Bewertung, Bedarf, Entwicklung; Zwischenergebnis. Forsttechnische Informationen, 54(9), 93-99.

Nick, L. \& Hofmann, R. (2005a). Deutliche Zuwächse im Neumaschinengeschäft bei Vollerntern und Tragschleppern. Forsttechnische Informationen, 57(8/9), 97-98.

Nick, L. \& Hofmann, R. (2005b). Forstmaschinenstatistik 2004: Deutlich mehr neue Vollernter und Tragschlepper verkauft. AFZ-DerWald, 60, 960-1159. 
Nurminen, T., Korpunen, H. \& Uusitalo, J. (2006). Time consumption analysis of the mechanized cut-to-length harvesting system. Silva Fennica, 40(2), 335-363.

Nurminen, T., Korpunen, H. \& Uusitalo, J. (2009). Applying the activity-based costing to cut-to-length timber harvesting and trucking. Silva Fennica, 43(5), 847-870.

Nuutinen, Y., Väätäinen, K., Asikainen, A., Prinz, R. \& Heinonen, J. (2010). Operational efficiency and damage to sawlogs by feed rollers of the harvester head. Silva Fennica, 44(1), 121-139.

Nuutinen, Y., Väätäinen, K., Heinonen, J., Asikainen, A. \& Röser, D. (2008). The accuracy of manually recorded time study data for harvester operation shown via simulator screen. Silva Fennica, 42(1), 63-72.

Oesten, G. \& Roeder, A. (2012). Management von Forstbetrieben. Institut für Forstökonomie der Universität Freiburg.

Ohrner, G. (1999). Wie wirtschaftlich arbeiten Harvester? Forst und Holz, 23, 727-732.

Ottaviani Aalmo, G., Kerstens, P. J., Belbo, H., Bogetoft, P., Talbot, B. \& Strange, N. (2020). Efficiency drivers in harvesting operations in mixed Boreal stands: a Norwegian case study. International Journal of Forest Engineering, 1-13.

Ottens, L. (2019). Prozessanalyse der Buchenholzrückung mittels Kombiforwarder (Bachelorarbeit). Georg-August-Universität Göttingen.

Ovaskainen, H. (2009). Timber harvester operators' working technique in first thinning and the importance of cognitive abilities on work productivity (Dissertation). University of Joensuu.

Ovaskainen, H., Uusitalo, J. \& Väätäinen, K. (2004). Characteristics and significance of a harvester operators' working technique in thinnings. International Journal of Forest Engineering, 15(2), 67-77.

Pausch, R. (1999). Wechselwirkungen zwischen Bestandsstruktur, biologischer Rationalisierung und forstlichen Verfahrenstechniken in Fichten-/Buchen-Betrieben Bayerns: Abschlussbericht zum Projekt A33. Lehrstuhl für Forstliche Arbeitswissenschaft und Angewandte Informatik der TU München.

Pausch, R. (2002). Ein System-Ansatz zur Untersuchung von Zusammenhängen zwischen Waldstruktur, Arbeitsvolumina und Kosten der technischen und biologischen Produktion in Forstrevieren ost- und nordbayerischer Mittelgebirge (Dissertation). Technische Universität München.

Pausch, R. (2007). Starkholz und Energieholz. Forsttechnische und wirtschaftliche Aspekte. AFZ-DerWald, 62(14), 746-748. 
Literaturverzeichnis

Pausch, R. \& Ponitz, K. (2002). Harvesterleistung und Hiebsbedingungen. Forst und Technik, 4, 10-14.

Pfeiffer, K., Abegg, B. \& Kuhn, P. (1978). Richtwerttabellen für die Holzhauerei und das Schichtholzrücken. Anleitung Nr. 8 der IG Industrieholz.

Pfohl, H.-C. (2004). Logistiksysteme: Betriebswirtschaftliche Grundlagen. Springer.

Plötner, O., Sieben, B. \& Kummer, T.-F. (2008). Kosten- und Erlösrechnung: Anschaulich, kompakt, praxisnah. Springer.

Pöhler, J. (2003). Einfluss der Arbeitsvorbereitung auf die Leistung von Kranvollerntern in der Nadeldurchforstung (Diplomarbeit). Technische Universität Dresden.

Porter, M. E. (1992). Wettbewerbsvorteile: Spitzenleistungen erreichen und behaupten. Campus-Verlag.

Pudack, T. (2006). Ansatzpunkte für den Strukturwandel in der Schweizer Forstwirtschaft. Schweizerische Zeitschrift für Forstwesen, 157(3/4), 73-81.

Purfürst, F. T. (2009). Der Einfluss des Menschen auf die Leistung von Harvestersystemen. Dr. Hut Verlag.

Purfürst, F. T. \& Erler, J. (2011). The human influence on productivity in harvester operations. International Journal of Forest Engineering, 22(2), 15-22.

Ranta, P. (2004). Possibilities to develop forest machine simulator based education with technology. Development Project in Forest Machine Based Training (ProForSim), Joensuu.

REFA-Fachausschuss Forstwirtschaft (1998). REFA-Fachbuchreihe Arbeitsgestaltung. Arbeitsstudien, Arbeitsorganisation und Qualitätsmanagement in der Forstwirtschaft. Verlag Institut für Arbeitsorganisation.

REFA-Fachausschuss Forstwirtschaft (2004). REFA-Fachbuchreihe Arbeitsgestaltung. Organisation in der Forstwirtschaft: Mit REFA-Methoden zu effizienten Arbeitsprozessen. ergonomia.

REFA-Verband für Arbeitsstudien (1978). Methodenlehre des Arbeitsstudiums. Hanser.

REFA Consulting AG (2020). Arbeitsaufgabe. REFA Lexikon. Zuletzt abgerufen am 28.10.2020 von https://refa-consulting.de/refa-lexikon/a/arbeitsaufgabe

Rencher, A. C. \& Schaalje, G. B. (2008). Linear models in statistics. Wiley-Interscience.

Richter, J. (1990). Kronentypen bei der Buche. Allgemeine Forst- und Jagdzeitung, 161(1), 11-15.

Röhrig, E., Bartsch, N., Lüpke, B. von \& Dengler, A. (2006). Waldbau auf ökologischer Grundlage. UTB. 
Roloff, A. (1985). Morphologie der Kronenentwicklung von Fagus sylvatica L. (Rotbuche) unter besonderer Berücksichtigung möglicherweise neuartiger Veränderungen (Dissertation). Georg-August-Universität Göttingen.

Roloff, A. (1988). Morphologie der Kronenentwicklung von Fagus sylvatica L. (Rotbuche) unter besonderer Berücksichtigung neuartiger Veränderungen: II. Strategie der Luftraumeroberung und Veränderungen durch Umwelteinflüsse. Flora, 180(3-4), 297-338.

Rosemann, M., Schwegmann, A. \& Delfmann, P. (2012). Vorbereitung der Prozessmodellierung. In J. Becker, M. Kugeler \& M. Rosemann (Hg.), Prozessmanagement (S. 47-111). Springer.

Rosenkranz, L. \& Seintsch, B. (2017). Ökonomische Analysen der Szenarien. AFZ-DerWald, 13, 24-26.

Rumpf, H. \& Petersen, R. (2008). Waldumbau mit Buche unter Berücksichtigung ihrer ökologischen Ansprüche. In Nordwestdeutsche Forstliche Versuchsanstalt (Hg.), Beiträge aus der Nordwestdeutschen Forstlichen Versuchsanstalt. Ergebnisse angewandter Forschung zur Buche (S. 193-219). Universitätsverlag Göttingen.

Rumpf, S., Nagel, M. \& Schmidt, M. (2011). Biomasseschätzfunktionen von Fichte, Kiefer, Buche, Eiche und Douglasie für Nordwestdeutschland. Ergebnisbericht des Forschungsvorhabens Möglichkeiten und Grenzen der Vollbaumnutzung. Fachagentur für nachwachsende Rohstoffe

Sachs, L. (1997). Angewandte Statistik: Anwendung statistischer Methoden. Springer.

Schardt, M. \& Sommer, W. (2007). Kalkulationshilfen für die Brennholzernte: Neues Zeitbedarfsmodell in schwächeren Fichtenbeständen. LWF aktuell, 61, 22-23.

Scheer, A.-W. (2002). ARIS - Vom Geschäftsprozess zum Anwendungssystem. Springer.

Schier, F. \& Weimar, H. (2017). Modellierung des Holzmarktes im WEHAM-Projekt. AFZ-DerWald, 13, 21-23.

Schlick, C., Luczak, H. \& Bruder, R. (2010). Arbeitswissenschaft. Springer.

Schluhe, M., Englert, H., Wördehoff, R., Schulz, C., Dieter, M. \& Möhring, B. (2018). Climate calculator for quantifying climate effects of forest enterprises based on data from forest management plans. Landbauforschung, 68(3-4), 67-87.

Schmidt, A. (2008). Kostenrechnung: Grundlagen der Vollkosten-, Deckungsbeitragsund Plankostenrechnung sowie des Kostenmanagements. Kohlhammer.

Schmidt, A. (2001). Prognosemodelle für ausgewählte Holzqualitätsmerkmale wichtiger Baumarten (Dissertation). Georg-August-Universität Göttingen. 
Literaturverzeichnis

Scholz, A. (2001). Informationelle Aspekte einer Geschäftsprozessanalyse: Mit dem Beispiel eines mittelständischen Unternehmens (Masterarbeit). Universität des Saarlandes.

Schöpfer, W., Kändler, G. \& Stöhr, D. (2004). Simulation einer wertoptimalen Holzeinteilung bei Vollerntereinsatz. Forst und Holz, 6, 263-268.

Schorr, M. (2000). Der Einfluss von Starkästen und Zwieseln auf die Leistung bei der Laubschwachholzernte. Forst und Holz, 55, 520-522.

Schöttle, R., Pfeil, C. \& Sauter, F. (1997). Leistung und Einsatzmöglichkeiten des Raupenharvesters in der Durchforstung. AFZ-DerWald, 52(22), 1179-1181.

Schraml, U. \& Härdter, U. (2002). Urbanität von Waldbesitzern und von Personen ohne Waldeigentum. AFZ-DerWald, 173(7), 140-145.

Schulte, C. (2011). Personal-Controlling mit Kennzahlen. Vahlen.

Schulz, H., Glos, P. \& Wegener, G. (1988). Überlegungen zu künftigen Anforderungen an Rohholz. Forstwissenschaftliches Centralblatt, 107(1), 317-325.

Schwegmann, A. (1999). Objektorientierte Referenzmodellierung: Theoretische Grundlagen und praktische Anwendung. Informationsmanagement und Controlling. Deutscher Universitätsverlag.

Sirén, M. \& Aaltio, H. (2003). Productivity and costs of thinning harvesters and harvesterforwarders. International Journal of Forest Engineering, 14(1), 39-48.

Speidel, G. (1952). Das Stückmassegesetz und seine Bedeutung für den internationalen Leistungsvergleich bei der Forstarbeit. Schriftenreihe des Instituts für forstliche Arbeitswissenschaft (Iffa). Gesellschaft für forstliche Arbeitswissenschaft e. V.

Speidel, G. (1984). Forstliche Betriebswirtschaftslehre. Parey.

Spellmann, H., Sutmöller, J. \& Meesenburg, H. (2007). Risikovorsorge im Zeichen des Klimawandels. AFZ-DerWald, 52, 1246-1249.

Spinelli, R., Hartsough, B. R. \& Magagnotti, N. (2010). Productivity standards for harvesters and processors in Italy. Forest Products Journal, 60(3), 226-235.

Spinelli, R., Magagnotti, N. \& Nati, C. (2009). Options for the mechanized processing of hardwood trees in mediterranean forests. International Journal of Forest Engineering, 20(1), 39-44.

Spinelli, R., Magagnotti, N. \& Nati, C. (2011). Work quality and veneer value recovery of mechanised and manual log-making in Italian poplar plantations. European Journal of Forest Research, 130(5), 737-744. 
Spinelli, R., Magagnotti, N., Sperandio, G., Cielo, P., Verani, S. \& Zanuttini, R. (2011). Cost and productivity of harvesting high-value hybrid poplar plantations in Italy. Forest Products Journal, 61(1), 64-70.

Spinelli, R., Owende, P. \& Ward, S. (2002). Productivity and cost of CTL harvesting of Eucalyptus globulus stands using excavator-based harvesters. Forest Products Journal, 51(1), 67-77.

Stampfer, K. (2001). Harvester Leistungsdaten - MHT Robin, Neuson 11002 HV, Impex Königstiger - Untersuchungsergebnisse aus Aufnahmen bei Geländeneigungen von 20 - 60\%. FPP Kooperationsabkommen Forst-Platte-Papier.

Stampfer, K. \& Steinmüller, T. (2004). Leistungsdaten Valmet 911.1 X3M. FPP Kooperationsabkommen Forst-Platte-Papier.

Statistisches Bundesamt [DESTATIS] (2017). Betriebsgrößenstruktur von Forstbetrieben. Zuletzt abgerufen am 28.10.2020 von https://www.destatis.de/DE/Themen/Branchen-Unternehmen/Landwirtschaft-Forstwirtschaft-Fischerei/WaldHolz/Tabellen/betriebsgroessenstruktur-forstbetrieben.html

Statistisches Bundesamt [DESTATIS] (2019). Zuletzt abgerufen am 28.10.2020 von Gesamteinschlag nach Holzartengruppen. https://www.destatis.de/DE/Themen/ Branchen-Unternehmen/Landwirtschaft-Forstwirtschaft-Fischerei/Wald-Holz/Tabellen/gesamteinschlag-holzartengruppen.html

Staud, J. (2013). Geschäftsprozessanalyse: Ereignisgesteuerte Prozessketten und objektorientierte Geschäftsprozessmodellierung für Betriebswirtschaftliche Standardsoftware. Springer.

Stoll, B., Burger, F. \& Blick, T. (2015). Es wächst und wächst und wächst: Schnellwachsende Baumarten - 20 Jahre Hackschnitzel und positive ökologische Ergebnisse. LWF aktuell, 105, 4-7.

Strandgard, M. \& Mitchell, R. (2020). Comparison of the productivity, cost and stem breakage of two- and three-machine harvest systems clearfelling a mature Pinus radiata stand. Australian Forestry, 83(2), 91-98.

Suadicani, K. \& Nordfjell, T. (2003). Operational aspects of row and selective thinning in the establishing of a shelterwood in a 50-year-old Norway spruce stand. International Journal of Forest Engineering, 14(1), 25-37.

Suchomel, C. (2010). Harvesting firewood from an aged oak coppice with a CTL hardwood harvester. International Forestry Review, 12(5), 13. 
Literaturverzeichnis

Suchomel, C., Becker, G. \& Pyttel, P. (2011). Fully mechanized harvesting in aged oak coppice stands. Forest Products Journal, 61(4), 290-296.

Suchomel, C., Spinelli, R. \& Magagnotti, N. (2012). Productivity of processing hardwood from coppice forests. Croatian Journal of Forest Engineering, 33(1), 39-47.

Sutmöller, J., Spellmann, H., Fiebiger, C. \& Albert, M. (2008). Der Klimawandel und seine Auswirkungen auf die Buchenwälder in Deutschland. In Nordwestdeutsche Forstliche Versuchsanstalt (Hg.), Beiträge aus der Nordwestdeutschen Forstlichen Versuchsanstalt. Ergebnisse angewandter Forschung zur Buche (S. 135-158). Universitätsverlag Göttingen.

Taylor, F. W. (1911). The principles of scientific management. Dover.

Thor, M. \& Frohm, S. (1997). Tvingande och selektivt uttag i gallring: Vad betyder maskinstorlek och stickvägsavstånd? Skogforsk.

Tufts, R. A. \& Brinker, R. W. (1993). Productivity of a Scandinavian cut-to-length system while second thinning pine plantations. Forest Products Journal, 43, 24-32.

Turney, P. B. B. (1992). Activity based costing. Zuletzt abgerufen am 28.10.2020 von https://www.cfoconsult.nl/wp-content/uploads/2012/10/ABC-supportdocument_Study-Unit-2.pdf

Väätäinen, K., Ala-Fossi, A. \& Ovaskainen, H. (2004). The importance of the harvester operator's know-ledge in CTL-harvesting operations. Development Project in Forest Machine Based Training (ProForSim), Joensuu.

Väätäinen, K., Ala-Fossi, A., Nuutinen, Y. \& Röser, D. (2006). The effect of single grip harvester's log bunching on forwarder efficiency. Baltic Forestry, 12(1), 64-69.

Vahrenkamp, R. (2005). Logistik: Management und Strategien. Oldenbourg.

Visser, R., Spinelli, R., Saathof, J. \& Fairbrother, S. (15. Juni 2009). Finding the 'SweetSpot' of Mechanised Felling Machines. Vortrag auf dem 32 ${ }^{\text {nd }}$ Annual Meeting of the Council on Forest Engineering (COFE 09), Kings Beach, CA, USA.

Voigt, K-I., Steven, M., von Weizsäcker, R.K. \& Horvath, M. (2018). Produktionsprozess. Gabler Wirtschaftslexikon. Zuletzt abgerufen am 28.10.2020 von https://wirtschaftslexikon.gabler.de/definition/produktionsprozess-45377/version-268671

Voigt, K.-I., Wohltmann, H.-W., Steven, M., von Weizsäcker, R. K. \& Horvath, M. (2018) Produktivität. Gabler Wirtschaftslexikon. Zuletzt abgerufen am 28.10.2020 von https://wirtschaftslexikon.gabler.de/definition/produktivitaet-46151/version269437 
Vom Brocke, J. \& Sonnenberg, C. (2011). Prozesstransparenz als Grundlage für das Management und Controlling von Geschäftsprozessen. Controlling \& Management, 55(S2), 55-68.

Von Arnswald, H. J. (1951). Höhenwachstum der Buche im nordwestdeutschen Raum. Allgemeine Forstzeitschrift, 173-175.

Von Bodelschwingh, E. (2006). Analyse der Rundholzlogistik in der deutschen Forst-und Holzwirtschaft-Ansätze für ein übergreifendes Supply Chain Management (Dissertation). Technische Universität München.

Von Wilpert, K., Bösch, B., Bastian, P., Zirlewagen, D., Hepperle, F., Holzmann, S., Puhlmann, H., Schäffer, J., Kändler, G. \& Sauter, U. H. (2011). Biomasse - Aufkommensprognose und Kreislaufkonzept für den Einsatz von Holzaschen in der Bodenschutzkalkung in Oberschwaben. Berichte Freiburger Forstliche Forschung.

Vossen, G. (Hg.). (1996). Informatik-Lehrbuch-Reihe. Geschäftsprozessmodellierung und Workflow-Management: Modelle, Methoden, Werkzeuge. Thomson.

Vötter, D. (2009). Sustainability Impact Assessment of the Forest-Wood-Supply-Chain based on a Process Modelling Approach - Comparison of Northern and Central Europe as an example (Dissertation). Albert-Ludwigs-Universität Freiburg im Breisgau.

Wang, J. \& Greene, W. D. (1999). An interactive simulation system for modeling stands, harvests, and machines. Journal of Forest Engineering, 10(1), 81-99.

Wang, J. \& LeDoux, C. B. (2003). Estimating and validating ground-based timber harvesting production through computer simulation. Forest Science, 49(1), 64-76.

Warkotsch, W. \& Ziesak, M. (1998). Die Holzerntekette - Probleme und Lösungsansätze. Institut für Forsttechnik Wien.

Weber, J. (2018). Prozesskostenrechnung. Gabler Wirtschaftslexikon. Zuletzt abgerufen am 28.10.2020 von https://wirtschaftslexikon.gabler.de/definition/prozesskostenrechnung-44779/version-268083

Wehner, T. \& Sauter, U. H. (2005). Aspekte der Holznutzung und der Technikfolgenabschätzung beim Waldumbau im südlichen Schwarzwald. K. von Teuffel, M. Baumgarten, M. Hanewinkel, U. H. Sauter, K. von Wilpert, W. Konold \& H. Spiecker (Hg.), Waldumbau: Für Eine Zukunftsorientierte Waldwirtschaft (S. 301-399). Springer.

Westermayer, T., Brogt, T. \& Oorschot, J. (2004). Forstdienstleister sind in der Mehrzahl Kleinstunternehmen. Haupttätigkeitsfelder Holzrücken und motormanuelle Holzernte. AFZ-DerWald, 23, 1247-1249. 
Literaturverzeichnis

Willems, S. (2015). Zur Abschätzung der Holzernte-Produktivität bei Optimierung von Bereitstellungsverfahren für Sortimente aus Laubholzkronen. Cuvillier Verlag.

Willmann, U., Mahler, G. \& Wurster, M. (2001). Güteansprache am stehenden Stamm im Rahmen der Bundeswaldinventur II. AFZ-DerWald, 56, 1024-1026.

Wöhe, G. \& Döring, U. (2010). Einführung in die allgemeine Betriebswirtschaftslehre.

Vahlen.

Wördehoff, R., Fischer, C. \& Spellmann, H. (2017). II. Cluster- und Kohlenstoffstudie Forst und Holz Niedersachsen. Universitätsverlag Göttingen.

Xue, L. \& Liang, H. (2009). Polynomial spline estimation for a generalized additive coefficient model. Scandinavian journal of statistics, theory and applications, 37(1), 26-46.

Ziesak, M., Bruchner, A. \& Hemm, M. (2004). Simulation technique for modelling the production chain in forestry. European Journal of Forest Research, 123(3), 239-244.

Zimmermann, W., Fries, H.-P. \& Hoch, G. (2003). Betriebliches Rechnungswesen. Oldenbourg. 


\section{Anhang}

Anhang 1: Orientierungstabelle für den elektronischen Anhang der Ausgleichsmodelle.

\begin{tabular}{ll}
\hline Dateiname & \multicolumn{1}{c}{ Inhalt } \\
\hline 2_1_volumen_aus- & Ausgleich fehlender Abschnittsdurchmesser und -längen für \\
gleich_he_1.R & die Versuchsfläche Hessen 1 \\
2_1_volumen_aus- & Ausgleich fehlender Abschnittsdurchmesser und -längen für \\
gleich_he_2.R & die Versuchsfläche Hessen 2 \\
2_1_volumen_aus- & Ausgleich fehlender Abschnittsdurchmesser und -längen für \\
gleich_ls_1.R & die Versuchsfläche Niedersachsen 1 \\
2_1_volumen_aus- & Ausgleich fehlender Abschnittsdurchmesser und -längen für \\
gleich_ls_2.R & die Versuchsfläche Niedersachsen 2 \\
2_1_volumen_aus- & Ausgleich fehlender Abschnittsdurchmesser und -längen für \\
gleich_ls_3.R & die Versuchsfläche Niedersachsen 3 \\
4_1_zeiten_aufberei- & $\begin{array}{l}\text { Ausgleich fehlender Zeitmessungen auf Abschnitts- und } \\
\text { tung_he_1.R }\end{array}$ \\
4_1_zeiten_aufberei- & $\begin{array}{l}\text { Auumebene für die Versuchsfläche Hessen } 1 \\
\text { tung_he_2.R }\end{array}$ \\
4_1_zeiten_aufberei- & $\begin{array}{l}\text { Baumebene für die Versuchsfläche Hessen } 2 \\
\text { tung_ls_1.R }\end{array}$ \\
4_1_zeiten_aufberei- & $\begin{array}{l}\text { Baumebene für die Versuchsfläche Niedersachsen 1 fehlender Zeitmessungen auf Abschnitts- und } \\
\text { tung_ls_2.R }\end{array}$ \\
\hline
\end{tabular}


Anhang

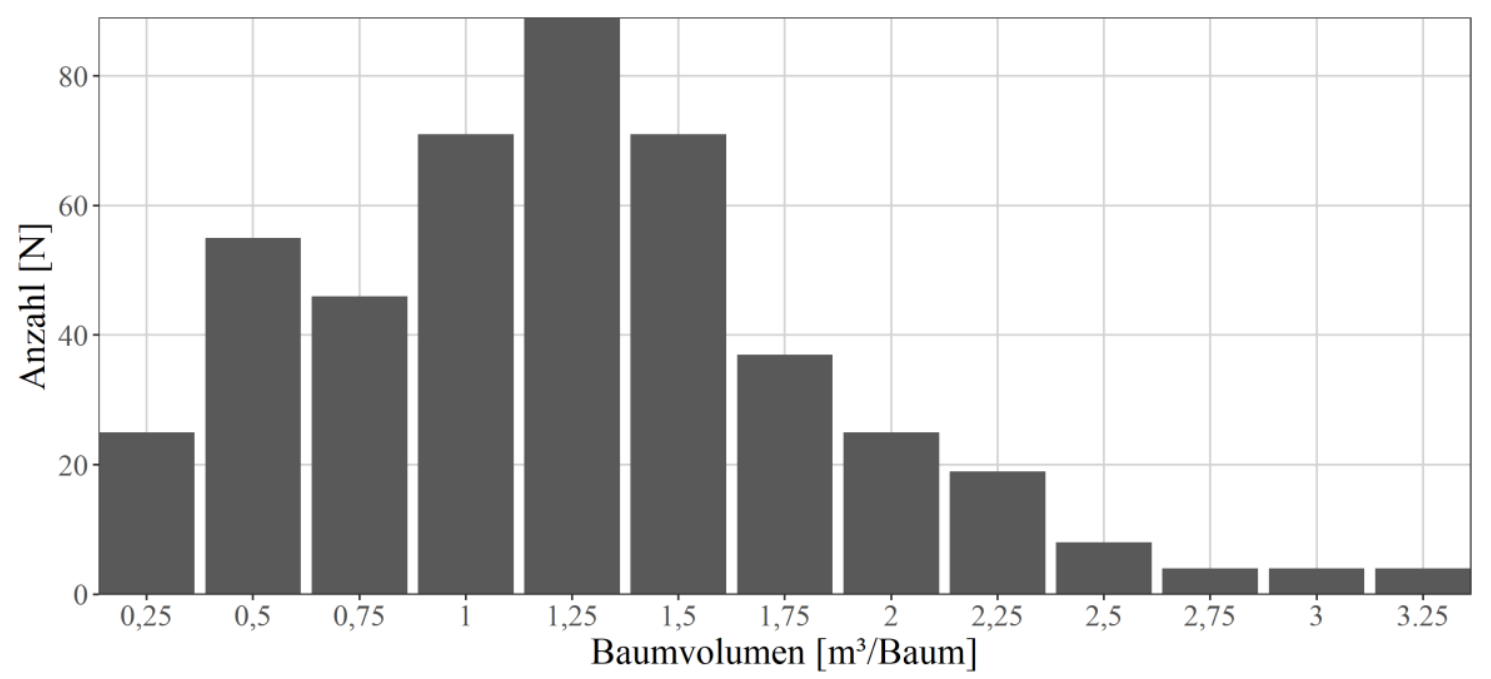

Anhang 2: Häufigkeitsverteilung der Stichprobe in Abhängigkeit zum Baumvolumen.; $N$ $=464$.

Anhang 3: Maximalmodell mit allen theoretischen Variablen für die lineare Regression des Zeitverbrauchs (Sekunden/Baum) der Aktivität Anfahrt und Positionierung; Regressionskoeffizient $b$, Standardfehler $S E$ und Signifikanzwert $p$. Legende siehe Variablenverzeichnis. $N=254 ; A I C=2.224 ; R^{2}=0,08$.

\begin{tabular}{lccc}
\hline Faktor & $\mathbf{b}$ & $\mathbf{S E}$ & $\mathbf{p}$ \\
$\beta_{0}$ & 21,73 & 2,92 & $<, 001^{* * *}$ \\
$\beta_{\text {Entf }}$ & 0,23 & 0,22 &, 306 \\
$\beta_{\text {Vol }}$ & 0,53 & 2,47 &, 829 \\
$\beta_{\text {Entf } \times \text { Vol }}$ & 0,19 & 0,18 &, 291 \\
$\beta_{\text {System } 2}$ & $-5,31$ & 3,56 &, 137 \\
$\beta_{\text {System3 }}$ & 1,53 & 2,76 &, 579 \\
\hline
\end{tabular}


Anhang 4: Maximalmodell mit allen theoretischen Variablen für die lineare Regression des Zeitverbrauchs (Sekunden/Baum) der Aktivität Greifen; Regressionskoeffizient $b$, Standardfehler $S E$ und Signifikanzwert $p$. Legende siehe Variablenverzeichnis. $\mathrm{N}=361$; $\mathrm{AIC}=1.720 ; R^{2}=0,61$.

\begin{tabular}{lccc}
\hline Faktor & $\mathbf{b}$ & $\mathbf{S E}$ & $\mathbf{p}$ \\
\hline$\beta_{0}$ & 5,23 & 0,70 & $<, 001^{* * *}$ \\
$\beta_{\text {Vol }}$ & 1,57 & 0,36 & $<, 001^{* * *}$ \\
$\beta_{\text {ZusatzG } 1}$ & 9,94 & 0,50 & $<, 001^{* * *}$ \\
$\beta_{\text {System } 2}$ & $-0,99$ & 0,42 &, $018^{*}$ \\
$\beta_{\text {System } 3}$ & 1,25 & 0,38 &, $001^{* *}$ \\
$\beta_{\text {Gasse }}$ & 0,00 & 0,04 &, 906 \\
$\beta_{\text {Gasse } \times \text { Vol }}$ & 0,00 & 0,29 &, 811 \\
$\beta_{\text {Qualität }}$ & 0,01 & 0,15 &, 951 \\
$\beta_{\text {KT2 }}$ & $-0,11$ & 0,44 &, 812 \\
$\beta_{\text {KT3 }}$ & $-0,19$ & 0,35 &, 593 \\
\hline
\end{tabular}

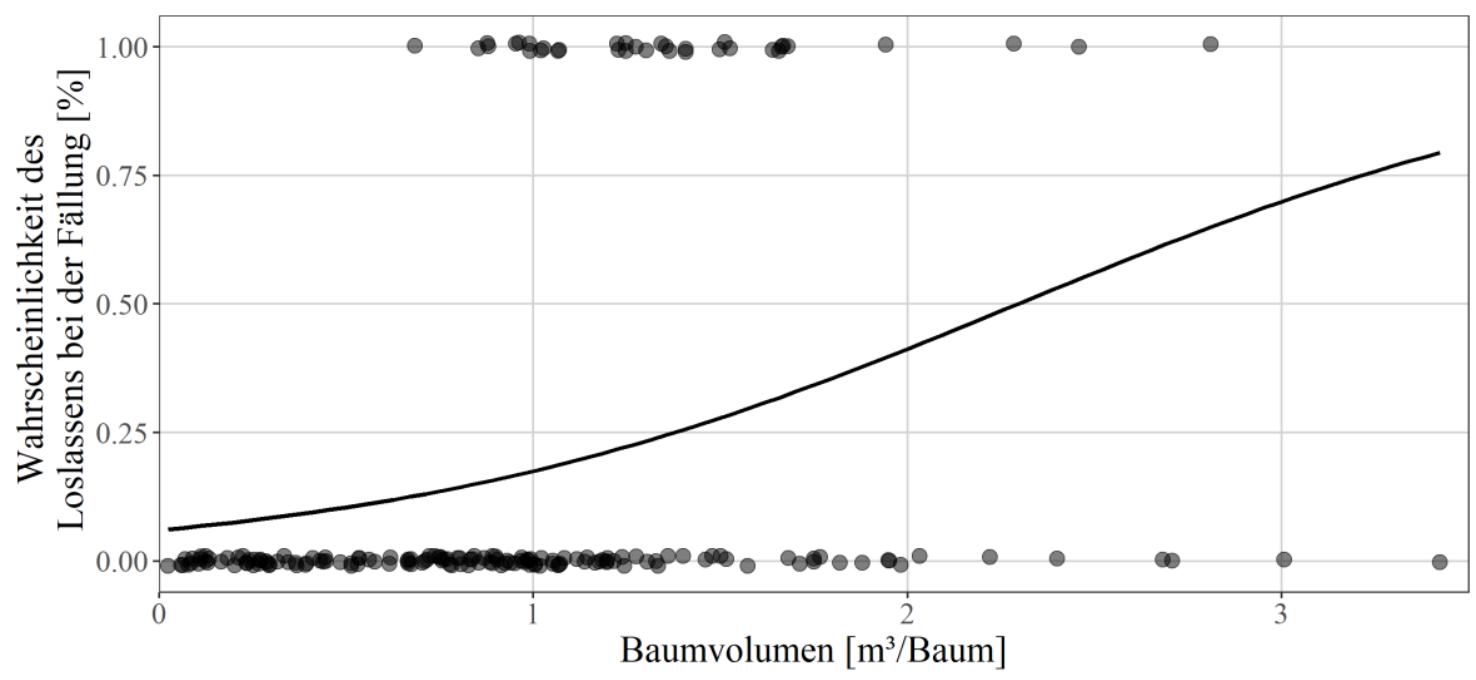

Anhang 5: Logistische Regression der Eintrittswahrscheinlichkeit für das Auftreten der Zusatzaktivität „Loslassen (L)“ innerhalb der Aktivität Fällung; $N=181$. 
Anhang

Anhang 6: Maximalmodell mit allen theoretischen Variablen für die quadratische Regression des Zeitverbrauchs (Sekunden/Baum) der Aktivität Fällung; Regressionskoeffizient $b$, Standardfehler $S E$ und Signifikanzwert $p$. Legende siehe Variablenverzeichnis. $\mathrm{N}=$ $361 ; \mathrm{AIC}=2.742 ; R^{2}=0,77$.

\begin{tabular}{lccc}
\hline Faktor & $\mathbf{b}$ & $\mathbf{S E}$ & $\mathbf{p}$ \\
\hline$\beta_{0}$ & $-5,52$ & 3,10 &, 076 \\
$\beta_{\text {Vol1 }}$ & 6,50 & 2,67 &, 015 \\
$\beta_{\text {Vol2 }}$ & 6,61 & 0,96 & $<, 001^{* * *}$ \\
$\beta_{S 1}$ & 4,83 & 2,73 &, 078 \\
$\beta_{Z 1}$ & 7,80 & 2,64 &, $003^{* *}$ \\
$\beta_{\text {L1 }}$ & 12,22 & 2,51 & $<, 001^{* * *}$ \\
$\beta_{\text {ZusatzF0 }}$ & $-0,43$ & 3,11 &, 891 \\
$\beta_{\text {Qualität }}$ & 1,17 & 0,61 &, 055 \\
$\beta_{\text {System } 2}$ & $-3,27$ & 1,74 &, 062 \\
$\beta_{\text {System } 3}$ & 8,17 & 1,57 & $<, 001 * * *$ \\
$\beta_{\text {Gasse }}$ & 0,06 & 0,11 &, 573 \\
\hline
\end{tabular}

Anhang 7: Maximalmodell mit allen theoretischen Variablen für die quadratische Regression des Zeitverbrauchs (Sekunden/Baum) der Aktivität Entasten auf Baumebene; Regressionskoeffizient $b$, Standardfehler $S E$ und Signifikanzwert $p$. Legende siehe Variablenverzeichnis. $\mathrm{N}=361 ; \mathrm{AIC}=2.934 ; R^{2}=0,78$.

\begin{tabular}{lccc}
\hline Faktor & $\mathbf{b}$ & $\mathbf{S E}$ & $\mathbf{p}$ \\
\hline$\beta_{0}$ & $-25,80$ & 11,52 &, $026^{*}$ \\
$\beta_{\text {Vol1 }}$ & 21,36 & 5,21 & $<, 001^{* * *}$ \\
$\beta_{\text {Vol2 }}$ & 3,25 & 1,58 &, $036^{*}$ \\
$\beta_{\text {Abschnitte }}$ & 3,72 & 1,72 &, $031^{*}$ \\
$\beta_{\text {Sorten }}$ & 7,79 & 5,54 &, 161 \\
$\beta_{\text {Qualität }}$ & 4,11 & 2,42 &, 090 \\
$\beta_{\text {KT2 }}$ & 1,85 & 2,43 &, 447 \\
$\beta_{\text {KT3 }}$ & $-1,78$ & 1,96 &, 365 \\
$\beta_{\text {System2 }}$ & $-2,56$ & 2,48 &, 304 \\
$\beta_{\text {System3 }}$ & 2,52 & 2,05 &, 221 \\
$\beta_{\text {Abschnitte } \times \text { Sorten }}$ & $-0,35$ & 0,44 &, 423 \\
$\beta_{\text {Abschnitte } \times \text { Qualität }}$ & $-0,28$ & 0,33 &, 389 \\
$\beta_{\text {Sorten } \times \text { Qualität }}$ & $-0,29$ & 1,16 &, 810 \\
\hline
\end{tabular}



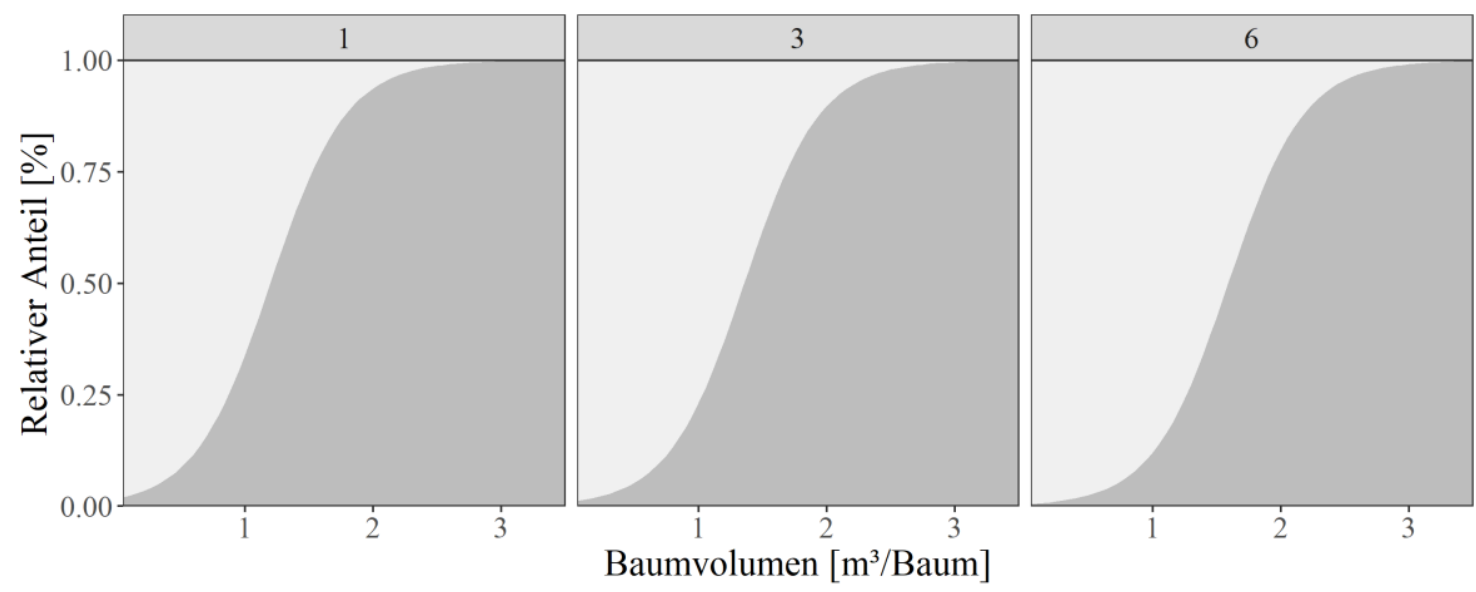

Anzahl Sorten IS $\quad$ SH IS

Anhang 8: Modellierte relative Anteile der Sortenanzahl in Abhängigkeit zum Baumvolumens und der Qualität in Versuchsfläche 1. Dargestellt für die Qualitäten 1,3 und 6. $N=$ 85.
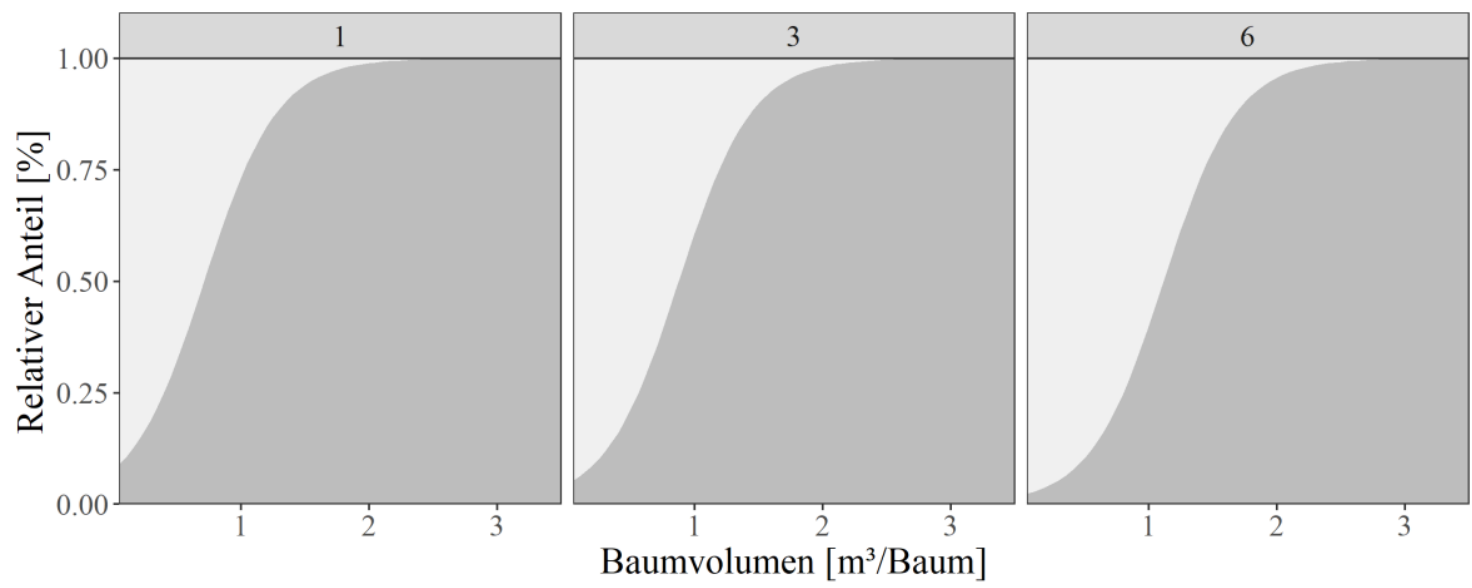

Anzahl Sorten IS PA IS

Anhang 9: Modellierte relative Anteile der Sortenanzahl in Abhängigkeit zum Baumvolumens und der Qualität in Versuchsfläche 3. Dargestellt für die Qualitäten 1,3 und 6. $N=$ 51. 
Anhang

Anhang 10: Maximalmodell mit allen theoretischen Variablen für die lineare Regression des Zeitverbrauchs (Sekunden/Baum) der Aktivität Umgreifen; Regressionskoeffizient $b$, Standardfehler $S E$ und Signifikanzwert $p$. Legende siehe Variablenverzeichnis. N = 134; $\mathrm{AIC}=1.444 ; R^{2}=0,88$.

\begin{tabular}{lccc}
\hline Faktor & $\mathbf{b}$ & $\mathbf{S E}$ & $\mathbf{p}$ \\
\hline$\beta_{\text {Vol }}$ & 10,41 & 3,98 &, $010^{*}$ \\
$\beta_{\text {Umgreifvorgänge }}$ & 13,17 & 2,65 & $<, 001^{* * *}$ \\
$\beta_{\text {Vol×Umgreifvorgänge }}$ & 0,26 & 1,25 &, 834 \\
$\beta_{\text {Qualität }}$ & 0,47 & 1,62 &, 773 \\
$\beta_{\text {System } 1}$ & $-17,85$ & 8,68 &, $042^{*}$ \\
$\beta_{\text {System } 2}$ & $-21,80$ & 9,32 &, $021^{*}$ \\
$\beta_{\text {System3 }}$ & 2,63 & 9,02 & 0,77 \\
\hline
\end{tabular}

Anhang 11: Maximalmodell mit allen theoretischen Variablen für die lineare Regression des Zeitverbrauchs (Sekunden/Baum) der Aktivität Kronenrest; Regressionskoeffizient $b$, Standardfehler $S E$ und Signifikanzwert $p$. Legende siehe Variablenverzeichnis. $\mathrm{N}=361 ; \mathrm{AIC}=2.251 ; R^{2}=0,35$.

\begin{tabular}{lccc}
\hline Faktor & $\mathbf{b}$ & $\mathbf{S E}$ & $\mathbf{p}$ \\
\hline$\beta_{0}$ & 2,02 & 1,32 &, 129 \\
$\beta_{\text {Vol }}$ & 2,77 & 0,48 & $<, 001^{* * *}$ \\
$\beta_{\text {ZusatzK1 }}$ & 14,51 & 2,51 & $<, 001^{* * *}$ \\
$\beta_{K T 2}$ & 2,38 & 0,94 &, $011^{*}$ \\
$\beta_{K T 3}$ & 4,43 & 0,72 & $<, 001^{* * *}$ \\
$\beta_{\text {Qualität }}$ & 0,14 & 0,30 &, 645 \\
$\beta_{\text {System } 2}$ & $-0,33$ & 0,88 &, 707 \\
$\beta_{\text {System3 }}$ & 0,99 & 0,65 &, 131 \\
\hline
\end{tabular}




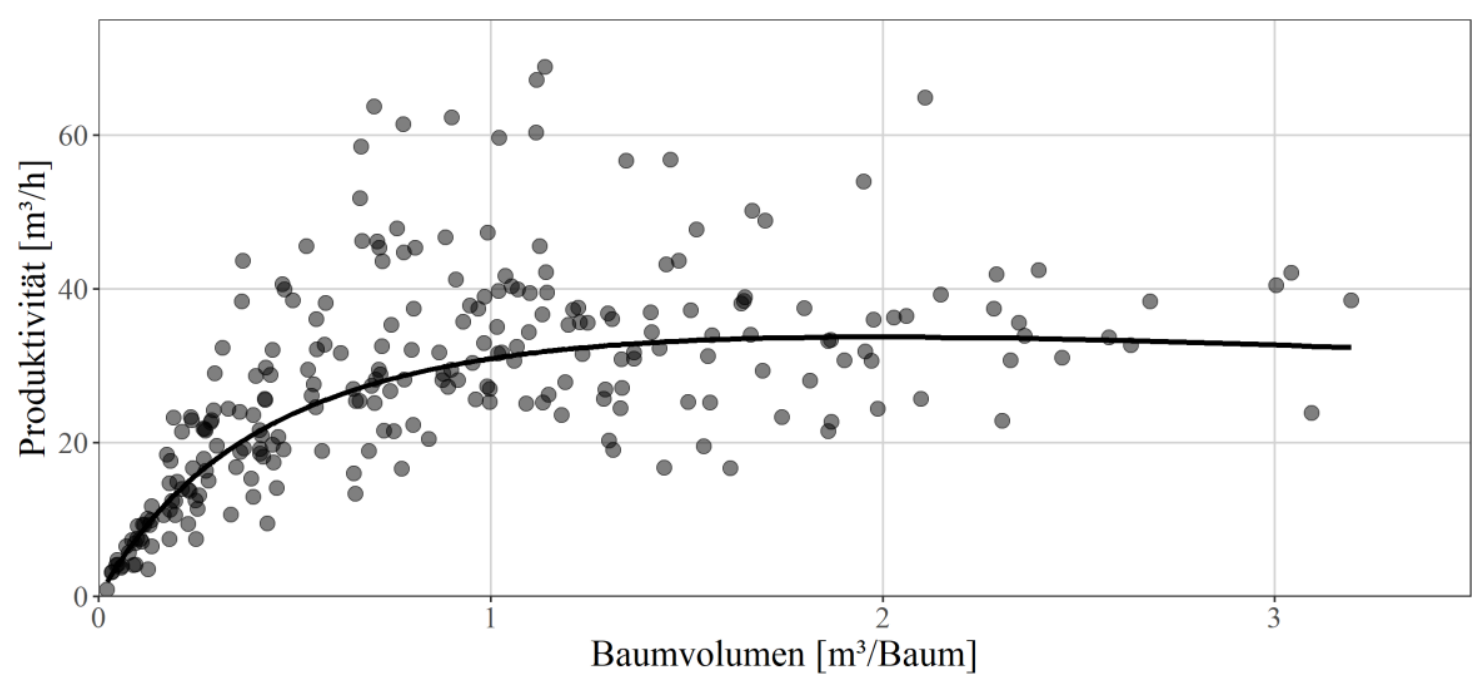

Anhang 12: Produktivität der mechanisierten Buchenaufarbeitung in Abhängigkeit vom Baumvolumen. $N=254 ; R^{2}=0,73$.

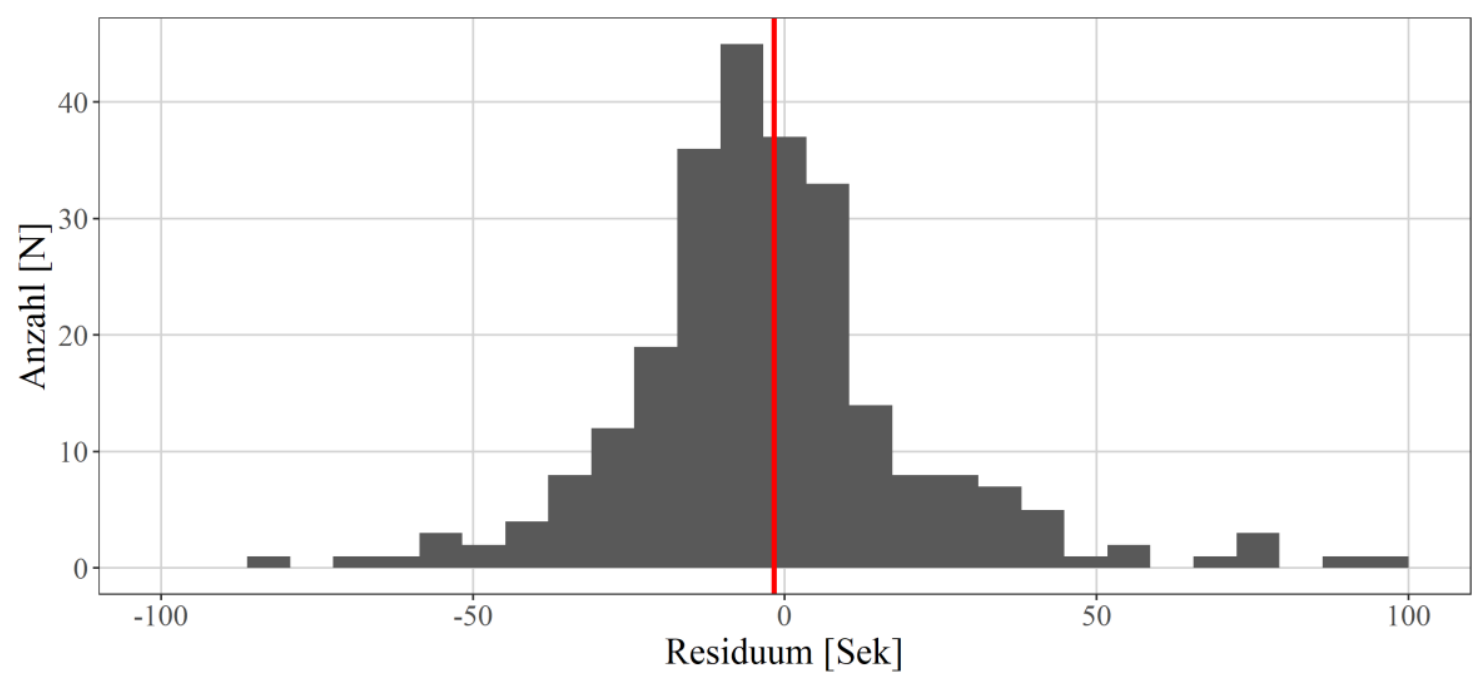

Anhang 13: Verteilung der Residuen des kumulierten Gesamtmodells (Formel 18). Die rote Line zeigt den Schwerpunkt der Verteilung; $N=254$. 
Anhang

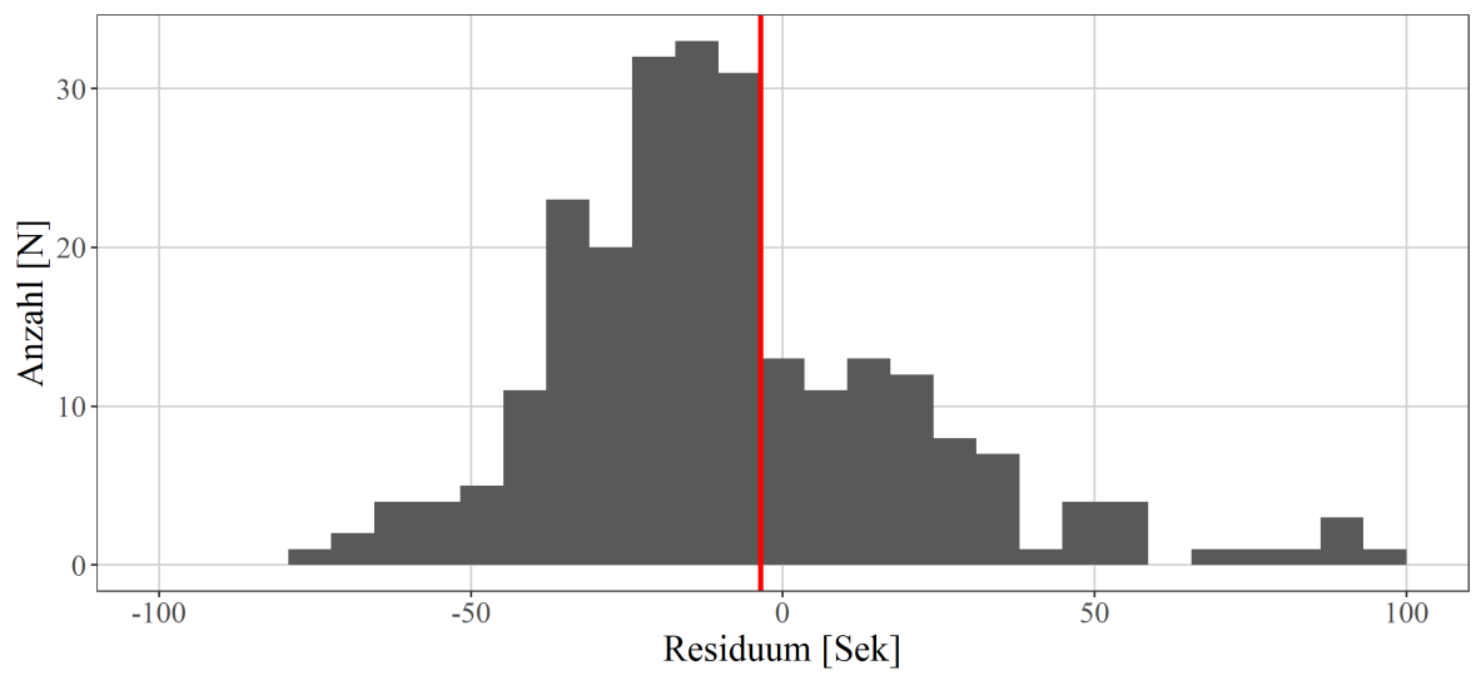

Anhang 14: Verteilung der Residuen des reduzierten Gesamtmodells (Formel 19). Die rote Line zeigt den Schwerpunkt der Verteilung. $N=254$.

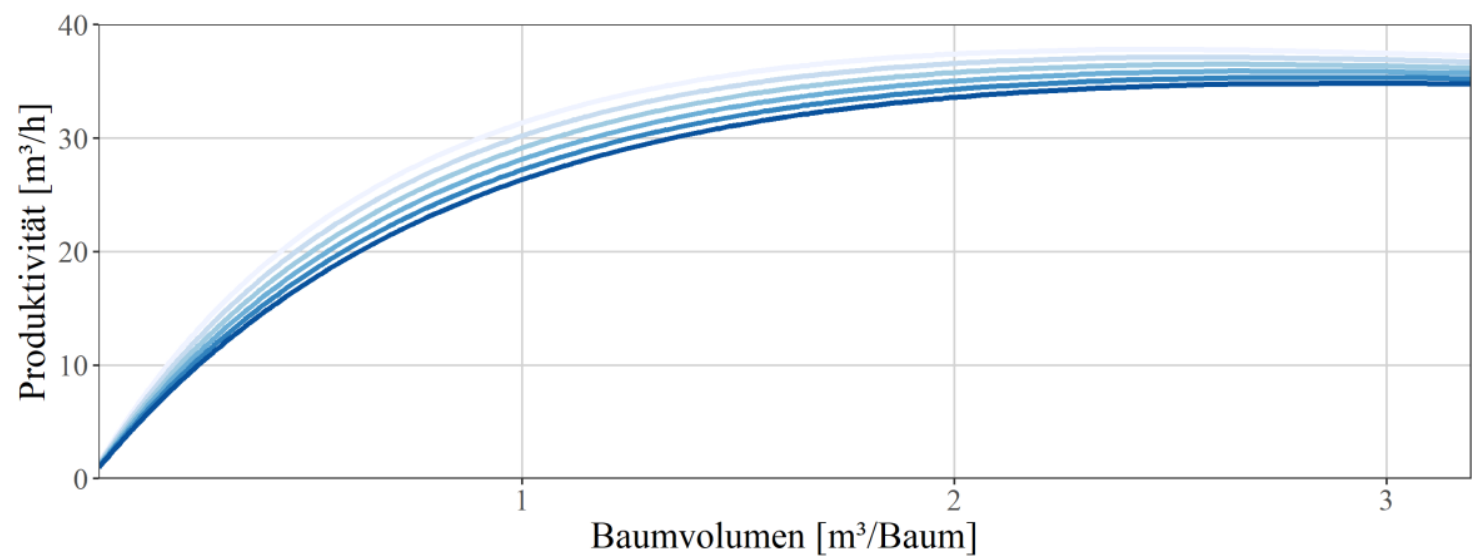

Entfernung zum Baum $[\mathrm{m}]-\begin{gathered}0-20-40 \\ -10=30-50\end{gathered}$

Anhang 15: Absolute Produktivität bei Anwendung des Produktivitätsmodells (Formel 20) in Abhängigkeit zum Baumvolumen und der Baumentfernung. 
Anhang 16: Übersicht über die verglichenen Laubholzmodelle zur Überprüfung der Forschungshypothese 5a.

\begin{tabular}{|c|c|}
\hline Modell & Funktion und Anmerkungen \\
\hline $\begin{array}{l}\text { Gulghör } 1994 \\
\text { Buche }+\end{array}$ & $\begin{aligned} R A Z\left(\frac{\min }{\text { Baum }}\right) & =0,76-0,0568 * B H D(\mathrm{~cm}) \\
& +0,00293 * B H D(\mathrm{~cm})^{2}\end{aligned}$ \\
\hline & $\begin{array}{l}\text {-Durchforstung von Buche mit Timberjack } 1070 \text { A (114 kW) } \\
\text {-Aggregat FMG } 746 \text { (max. } 45 \mathrm{~cm}), 10 \mathrm{~m} \text { Kran } \\
\text {-Mittlerer BHD Buche } 28 \mathrm{~cm}\left(\max .1,5 \mathrm{~m}^{3} \text { Baumvolumen) }\right. \\
\text {-Stärkere Geländeneigung (20 bis max. } 40 \%) \\
\text {-wipfelschäftige Buche }\end{array}$ \\
\hline $\begin{array}{l}\text { Gulghör } 1994 \\
\text { Buche - }\end{array}$ & $\begin{array}{l}\qquad \begin{array}{l}R A Z\left(\frac{\min }{\text { Baum }}\right)=1,32-0,0847 * B H D(\mathrm{~cm}) \\
+0,00402 * B H D(\mathrm{~cm})^{2}\end{array} \\
\text {-gleiche Anmerkungen wie beim Gulghör Buche + Modell } \\
\text {-gültig für zwieselige Buchen }\end{array}$ \\
\hline $\begin{array}{l}\text { Cremer } 2008 \\
\text { Laubholz }\end{array}$ & $\begin{array}{l}\text { Produktivität }\left(\frac{m^{3}}{M A S_{0}}\right)= \\
\qquad \begin{aligned}+1,466 * B H D(\mathrm{~cm}) \\
+\end{aligned} \\
\text {-Durchforstung von Laubholzbeständen (bis } 1 \mathrm{~m}^{3} \text { Baumvolumen) } \\
\text { mittels Timberjack } 1270(132 \mathrm{~kW})\end{array}$ \\
\hline $\begin{array}{l}\text { Nurminen et al. } \\
2006 \text { Birke }\end{array}$ & $\begin{array}{l}\qquad \begin{array}{l}\text { Produktivität }\left(\frac{m^{3}}{M A S_{0}}\right)=2,368+96,126 * V o l \\
-60,694 * V_{o l}^{2}\end{array} \\
\text {-Ernte von Birkenaltholzbeständen (bis } 1 \mathrm{~m}^{3} \text { Baumvolumen) } \\
\text {-einfache Geländebedingungen } \\
\text {-keine weiteren Angaben zum eingesetzten Radharvester }\end{array}$ \\
\hline
\end{tabular}

Anhang 17: Quadratische Regression des BHD $(\mathrm{cm})$ in Abhängigkeit zum Baumvolumen $\left(\mathrm{m}^{3}\right) \cdot N=254, R^{2}=0,95$.

\begin{tabular}{lccc}
\hline Faktor & b & SE & p \\
\hline$\beta_{V o l}$ & 46,47 & 1,06 & $<, 001 * * *$ \\
$\beta_{V o l^{2}}$ & $-10,55$ & 0,54 & $<, 001 * * *$ \\
\hline
\end{tabular}


Anhang

Anhang 18: Übersicht über die verglichenen Nadelholzmodelle zur Überprüfung der Forschungshypothese $5 \mathrm{~b}$.

\begin{tabular}{lr}
\hline Modell & Funktion und Anmerkungen \\
\hline Alam et al. & Produktivität $\left(\frac{m^{3}}{M A S_{0}}\right)=74,58+71,45 * \ln (\mathrm{Vol})$ \\
2014 Kiefer + & -Ernte von Kieferaltbeständen mit Valmet 475EX $(114 \mathrm{~kW})$ \\
& \\
& -Aggregat Rosin 997 \\
& -Mittleres Baumvolumen $1,8 \mathrm{~m}^{3}$ \\
\hline
\end{tabular}

Alam et al. $\quad$ Produktivität $\left(\frac{m^{3}}{M A S_{0}}\right)=70,59+48,16 * \ln (\mathrm{Vol})$
2014 Kiefer -

-gleiche Anmerkungen wie beim Alam et al. 2014 Kiefer + Modell

-Mittleres Baumvolumen 2,4 $\mathrm{m}^{3}$

$\begin{aligned} \text { Cremer } 2008 & \text { Produktivität }\left(\frac{m^{3}}{M A S_{0}}\right) & =-0,022 * B H D(\mathrm{~cm})^{2} \\ \text { Fichte } & & +1,466 * B H D(\mathrm{~cm})-0,678\end{aligned}$

-Durchforstung von Fichtenbeständen mit

Timberjack $1270(132 \mathrm{~kW})$

\begin{tabular}{|c|c|}
\hline $\begin{array}{l}\text { Nurminen et al. } \\
2006 \text { Fichte }\end{array}$ & $\begin{aligned} \text { Produktivität }\left(\frac{m^{3}}{M A S_{0}}\right) & =4,067+78,623 * \text { Vol } \\
& -18,507 * V_{o l}^{2}\end{aligned}$ \\
\hline & $\begin{array}{l}\text {-Ernte von Fichtenaltholzbeständen } \\
\text {-einfache Geländebedingungen } \\
\text {-keine weiteren Angaben zum eingesetzten Radharvester }\end{array}$ \\
\hline $\begin{array}{l}\text { Nurminen et al. } \\
2006 \text { Kiefer }\end{array}$ & $\begin{array}{l}\qquad \begin{array}{l}\text { Produktivität }\left(\frac{m^{3}}{M A S_{0}}\right)=1,383+99,375 * V o l \\
-39,824 * V_{o l}^{2}\end{array} \\
\text {-Ernte von Kiefernaltholzbeständen } \\
\text {-einfache Geländebedingungen } \\
\text {-keine weiteren Angaben zum eingesetzten Radharvester }\end{array}$ \\
\hline
\end{tabular}


Anhang 19: Regressionskoeffizient $b$, Standardfehler $S E$ und Signifikanzwert $p$ für die quadratische Regression des Zeitverbrauchs (Sekunden/Baum) des analytischen Gesamtmodells für den Prozess der mechanisierten Buchenaufarbeitung; $N=361 ; A I C=1.223$. Legende siehe Variablenverzeichnis.

\begin{tabular}{lccc}
\hline Faktor & $\mathbf{b}$ & $\mathbf{S E}$ & $\mathbf{p}$ \\
\hline$\beta_{0}$ & 19,19 & 5,31 & $<, 001^{* * *}$ \\
$\beta_{\text {Vol1 }}$ & 36,58 & 9,29 & $<, 001^{* * *}$ \\
$\beta_{\text {Vol2 }}$ & 12,20 & 3,21 & $<, 001^{* * *}$ \\
$\beta_{\text {Entf }}$ & 0,31 & 0,18 &, 087 \\
$\beta_{\text {KT2 }}$ & 8,37 & 6,14 &, 174 \\
$\beta_{\text {KT3 }}$ & 10,77 & 5,10 &, $036^{*}$ \\
$\beta_{\text {System2 }}$ & 1,11 & 5,84 &, 849 \\
$\beta_{\text {System3 }}$ & 33,49 & 4,62 & $<, 001^{* * *}$ \\
$\beta_{\text {Umgreifvorgänge }}$ & 12,28 & 1,45 & $<, 001^{* * *}$ \\
$\beta_{\text {ZusatzF0 }}$ & $-69,54$ & 29,95 &, $021^{*}$ \\
$\beta_{\text {S1 }}$ & 83,86 & 31,69 &, $009^{* *}$ \\
$\beta_{Z 1}$ & 82,13 & 30,19 &, $007^{* *}$ \\
$\beta_{\text {L1 }}$ & 75,33 & 29,91 &, $012^{*}$ \\
$\beta_{\text {ZusatzG } 1}$ & 6,01 & $<, 001^{* * *}$ \\
$\beta_{\text {ZusatzK1 }}$ & 20,38 & 20,86 &, $019^{*}$ \\
\hline
\end{tabular}

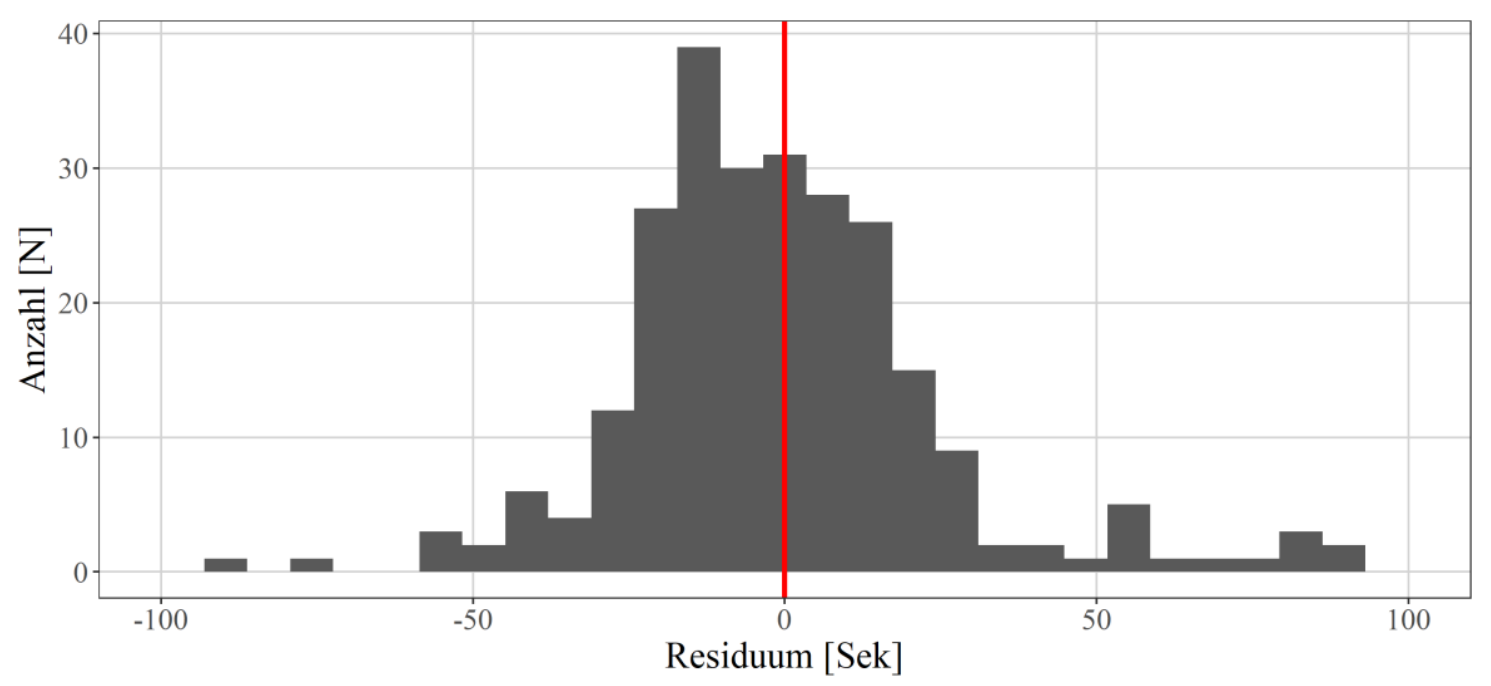

Anhang 20: Verteilung der Residuen des direkt geschätzten Gesamtmodells (Anhang 19). Die rote Line zeigt den Schwerpunkt der Verteilung. $N=254$. 
Anhang

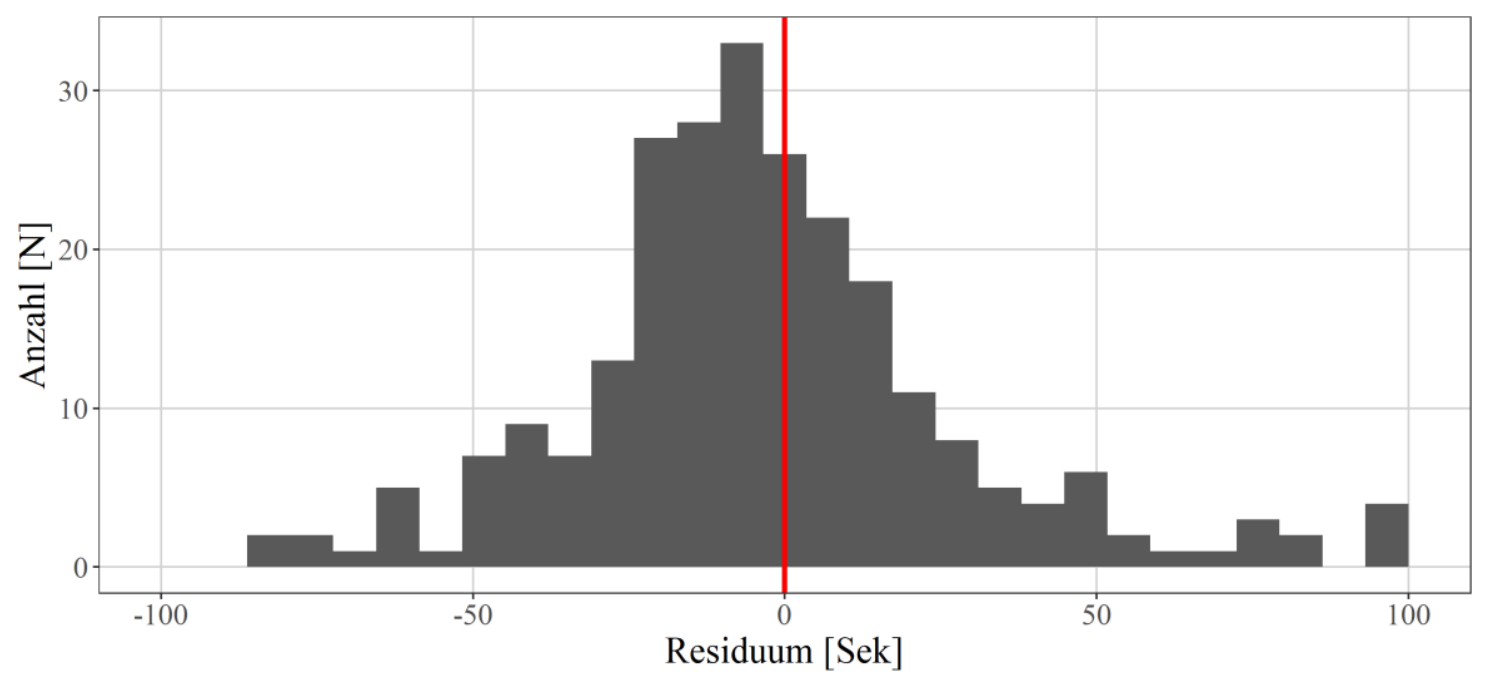

Anhang 21 Verteilung der Residuen des reduzierten direkt geschätzten Modells (Formel 24). Die rote Line zeigt den Schwerpunkt der Verteilung. $N=254$. 


\section{Eigenständigkeitserklärung}

Hiermit erkläre ich, dass die vorliegende Arbeit ohne unzulässige Hilfe und ohne Benutzung anderer als der angegebenen Hilfsmittel selbständig angefertigt wurde und dass die aus fremden Quellen direkt oder indirekt übernommenen Gedanken in der Arbeit als solche kenntlich gemacht worden sind. Alle genutzten fremden Quellen sind im Literaturverzeichnis gelistet.

Fabian Böttcher,

Göttingen, den 01.11.2020 


\section{Lebenslauf}

\section{Fabian Böttcher}

Geburtsdatum 22.04.1992 in Leinefelde

Staatsangehörigkeit deutsch

$03.2017-12.2020 \quad$ Wissenschaftlicher Mitarbeiter

Abteilung Forstökonomie, Georg-August-Universität Göttingen

seit 04.2017

\section{Forstwissenschaften und Waldökologie (Promotion)}

Georg-August-Universität Göttingen

1. Gutachter: Prof. Dr. B. Möhring (Forstökonomie)

2. Gutachter: Prof. Dr. D. Jaeger (Arbeitswissenschaft)

04.2015 - 02.2017 Management von Forstbetrieben (Master of Science)

Fachhochschule Erfurt

$10.2011-03.2015$ Forstwirtschaft und Waldökologie (Bachelor of Science)

Fachhochschule Erfurt 